\section{To: (Receiving Organization) \\ DISTRIBUTION \\ 5. Proj./Prog./Dept./Div.: \\ INTERIM STABILIZATION}

8. Originator Remarks:

ATTACHED IS THE SOETWARE PROGRAM DOCUMENTATION EOR PUMPING

INSTRUMENTATION AND CONTROL SKID "N".
4. Related EDT No.:
N/A
7. Purchase Order No.:
N/A
9. Equip./Component No.:

$\mathrm{N} / \mathrm{A}$

10. System/Bldg./Facility:

$241-G$

12. Major Assm. Dwg. No.:

$\mathrm{N} / \mathrm{A}$

13. Permit/Permit Application No.:

$\mathrm{N} / \mathrm{A}$

14. Required Response Date:

$03 / 24 / 00$

\begin{tabular}{|c|c|c|c|c|c|c|c|c|}
\hline 15. & & DATA TR & ANSMITT & & (F) & (G) & (H) & (l) \\
\hline $\begin{array}{l}\text { (A) } \\
\text { Item } \\
\text { No. }\end{array}$ & (B) Document/Drawing No. & $\begin{array}{l}\text { (C) Sheet } \\
\text { No. }\end{array}$ & (D) Rev. & (E) Title or Description of Data Transmitted & $\begin{array}{c}\text { Approval } \\
\text { Desig- } \\
\text { nator }\end{array}$ & $\begin{array}{l}\text { Reason } \\
\text { for Trans- } \\
\text { mittal }\end{array}$ & $\begin{array}{c}\text { Origi- } \\
\text { natoor } \\
\text { Dispo- } \\
\text { stion }\end{array}$ & $\begin{array}{l}\text { Receiv- } \\
\text { er } \\
\text { Dispo- } \\
\text { sition }\end{array}$ \\
\hline 1 & RPP-5492 & $\mathrm{N} / \mathrm{A}$ & 0 & PLC/DTAM SOFTWARE PROGRAMS & SIA & 1 & 1 & 1 \\
\hline & & & & FOR PUMPING & & & & \\
\hline & & & & INSTRUMENTATION AND & & & & \\
\hline & & & & CONTROL SKID "N" & & & & \\
\hline & & & & & & & & \\
\hline & & & & & & & & \\
\hline & & & & & & & & \\
\hline
\end{tabular}

16.

\begin{tabular}{|c|l|}
\hline Approval Designator (F) & \\
\hline $\begin{array}{c}\text { E, S, Q, D OR N/A } \\
\text { (See WHC-CM-3-5, } \\
\text { Sec. 12.7) }\end{array}$ & $\begin{array}{l}\text { 1. Approval } \\
\text { 2. Release } \\
\text { 3. Information }\end{array}$ \\
\hline
\end{tabular}

17.

Reason for Transmittal (G)

4. Review

5. Post-Review

6. Dist. (Receipt Acknow. Required)

KEY
SIGNATURE/DISTRIBUTION

(See Approval Designator for required signatures)

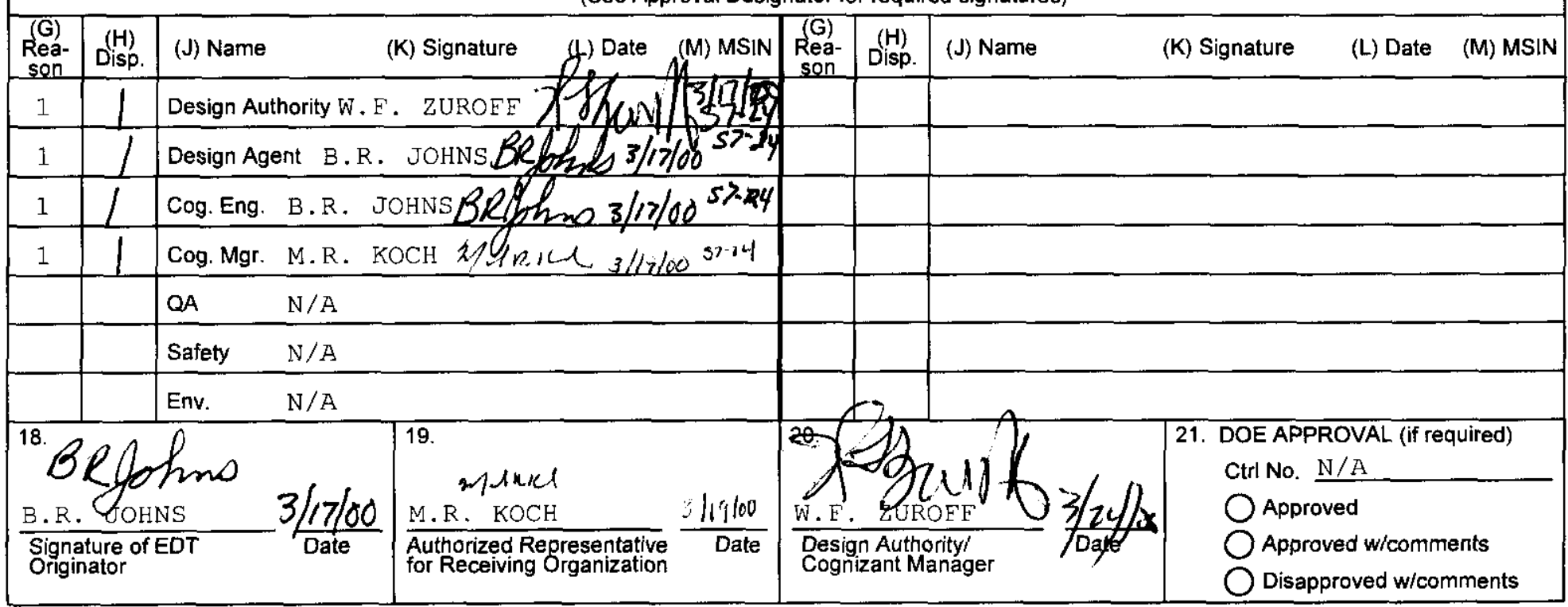




\title{
PLC/DTAM SOFTWARE PROGRAMS FOR PUMPING INSTRUMENTATION AND CONTROL SKID "N"
}

\author{
M. R. ROCH \\ CH2MHILL HANFORD GROUP, INC \\ Richland, WA 99352 \\ U.S. Department of Energy Contract DE-AC06-99RL14047
EDT/ECN: $624899 \quad$ UC:
Cost Center: 74D00 Charge Code: 103361 \\ B\&R Code: Ew3120071 Total Pages: 175 \\ Key Words: PLC, DTAM, LADDER LOGIC, PICS, SKID, INTERIM STABILIZATION, \\ SALT WELL

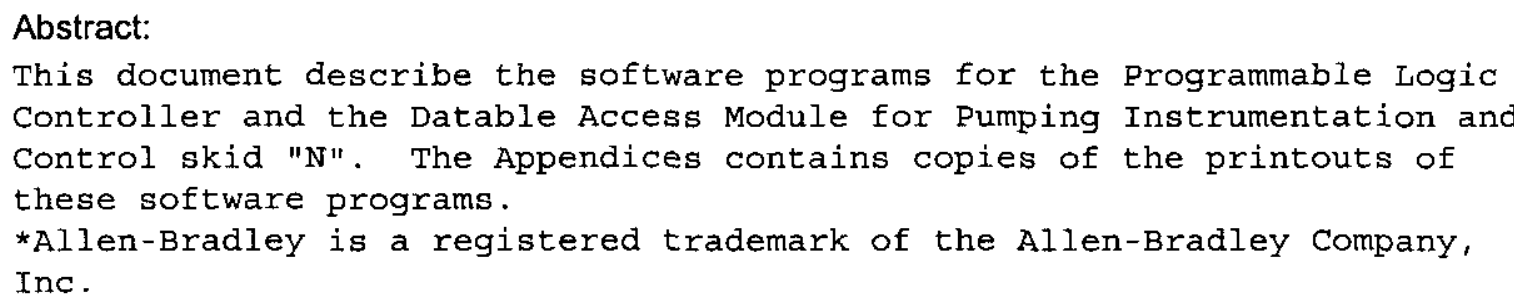

TRADEMARK DISCLAIMER. Reference herein to any specific commercial product, process, or service by trade name, trademark, manufacturer, or otherwise, does not necessarily constitute or imply its endorsement, recommendation, or favoring by the United States Government or any agency thereof or its contractors or subcontractors.

Printed in the United States of America. To obtain copies of this document, contact: Document Control Services, P.O. Box 950, Mailstop H6-08, Richland WA 99352, Phone (509) 372-2420; Fax (509) 376-4989.
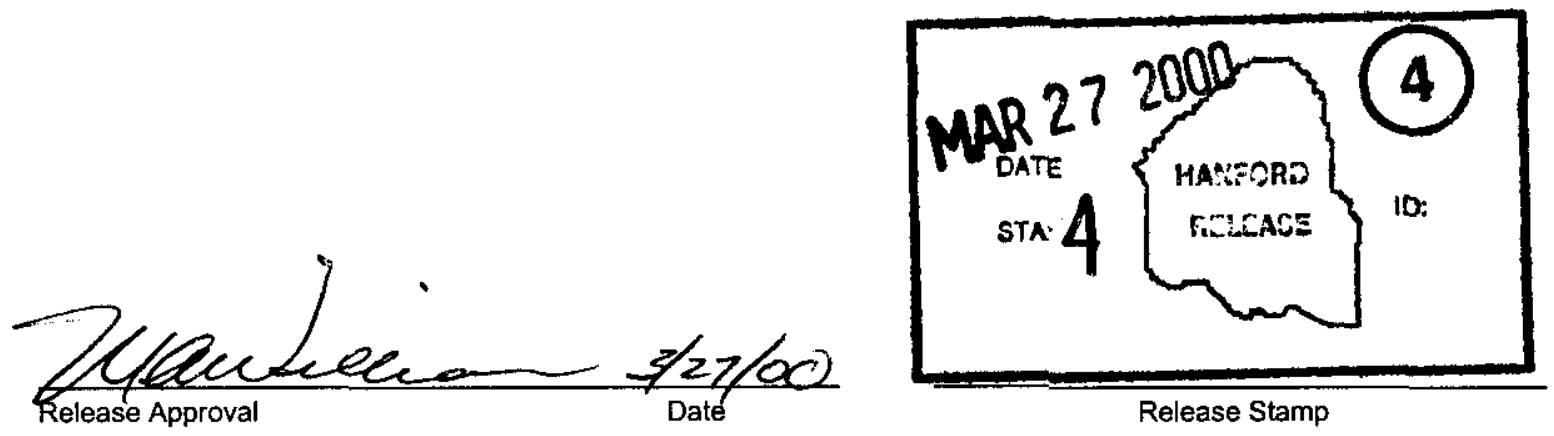

Release Stamp 
RPP-5492

REVISION 0

\section{TABLE OF CONTENTS}

1.0 INTRODUCTION

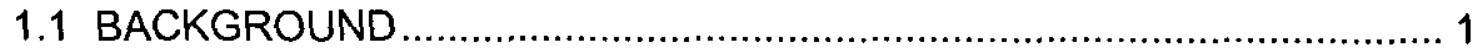

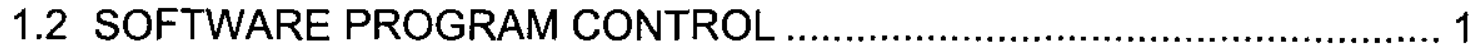

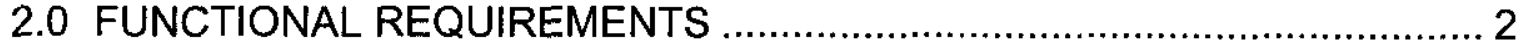

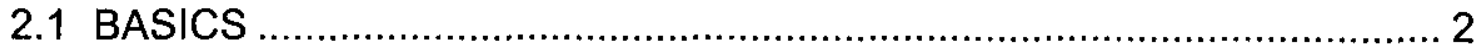

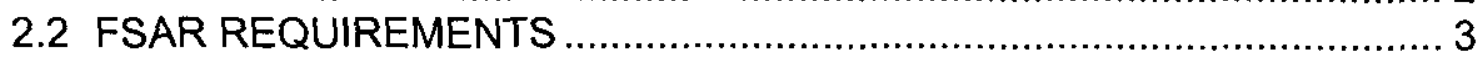

2.3 COMPLIANCE WITH FSAR FUNCTIONAL REQUIREMENTS AND

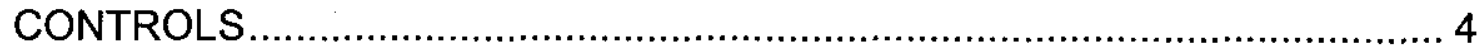

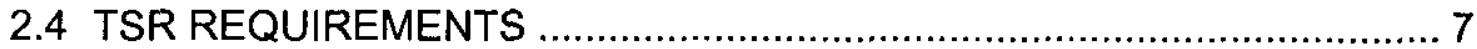

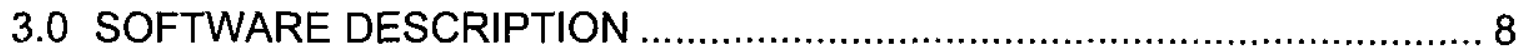

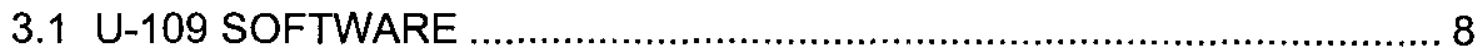

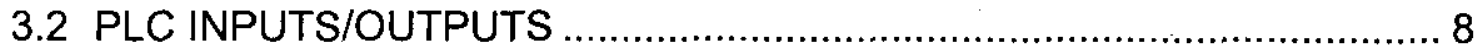

3.3 DTAM DISPLAYS, DATA ENTRY AND ALARMS ............................. 10

3.4 JET PUMP INTERLOCKS ........................................................... 11

3.5 INTERLOCK and ALARM CONDITIONS …........................................ 13

3.6 PIC SKID COMMUNICATIONS ........................................................ 15

4.0 SOFTWARE PROGRAM DESCRIPTION ......................................... 16

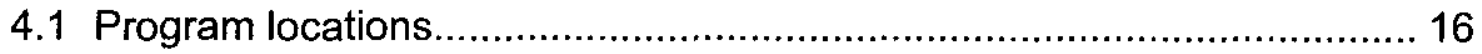

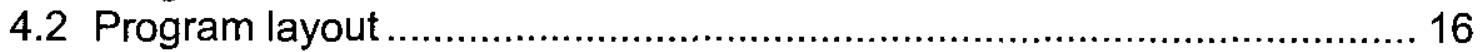

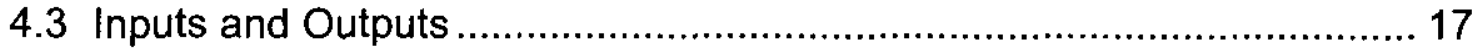

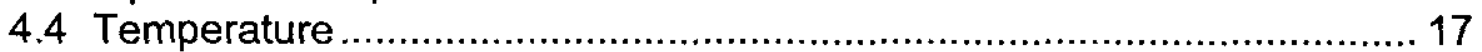

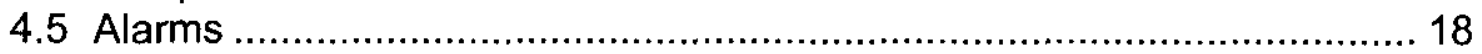

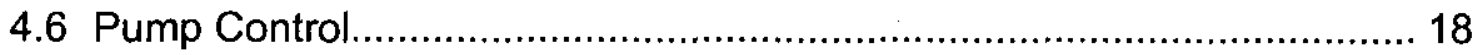

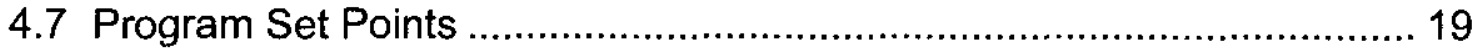

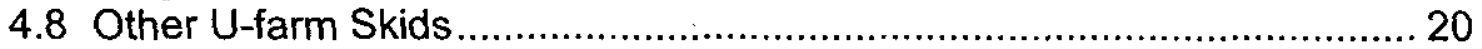

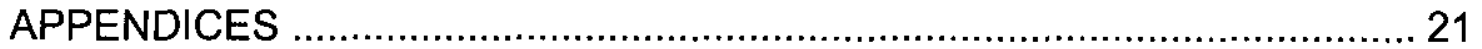


RPP-5492

REVISION 0

\section{ACRONYMS AND ABBREVIATIONS}

PLC - Programmable Logic Controller

DTAM - Data Table Access Module

OCS - Operator Control Station

FSAR - Basis for Interim Operation

TSR - Technical Safety Requirements

COB - Clean Out Box

LFL - Lower Flammability Limit

LCO - Limiting Condition for Operation

DOV - Diaphragm Operated Valve

RFPT - Recirculation Flush Pressure Transducer

JFPT - Jumper Flush Pressure Transducer

FGM - Flammable Gas Monitor

CGM - Combustible Gas Monitor

PXPT - Pump Transfer Pressure Transducer

OPT - Operational Test Procedure

ATP - Acceptance Test Procedure 
RPP-5492

REVISION 0

\section{PLC/DTAM SOFTWARE PROGRAMS FOR PUMPING INSTRUMENTATION AND CONTROL SKID "N"}

\subsection{INTRODUCTION}

\subsection{BACKGROUND}

This document describes the software programs for the Allen-Bradley SLC 500 programmable logic controller (PLC) and the Allen-Bradley DTAM PLUS operator interface module used on Pumping Instrumentation and Control (PIC) skid "N". The software programs for the SLC 500 and DTAM Plus are based on the core programs provided by Allen-Bradley. The PLC and DTAM software programs on skid "D" for SX-104 are the baseline programs. These baselines have been tailored for U-farm skids. The skid "N" program for U-109 is similar to the skid "M" program for U-102. An Acceptance Test Procedure (ATP) and an Operational Test Procedure (OTP) verify that the software programs meet the specific requirements for $U-109$ pumping.

This document represents the final PLC and DTAM programs for PIC skid "N" at U-109. These programs were printed out after the performance of the OTP. The OTP acts as the final qualification test for the software programs. Functional requirements and details of the PLC ladder logic are described in this document. The final programs entered into the PLC and DTAM Plus are included as Appendices to this document.

\subsection{SOFTWARE PROGRAM CONTROL}

This is the official software control document for the PLC and DTAM program for $\mathrm{PIC}$ skid " $\mathrm{N}$ ". Changes to the programs shall be documented as changes and/or revisions to this document. This is to comply with HNF-5034 for software configuration control for the PIC skid software programs. 
RPP-5492

REVISION 0

\subsection{FUNCTIONAL REQUIREMENTS}

\subsection{BASICS}

The basic functional requirements for the software program are identified below.

2.1.1 Control the jet pump and dilution pump

2.1.2 Monitor conditions and provide alarm and pump shutdown for off normal conditions

2.1.3 Provide PIC status information to the Operator Control Station (OCS) and other PIC skids that are on the same transfer line

2.1.4 Provide flow information on the amount of waste being pumped

Functional requirements PIC skid "N" must meet are in the Authorization Basis documents which include the Final Safety Analysis Report, HNF-SD-WM-SAR067, Revision 1; and Technical Safety Requirements, HNF-SD-WM-TSR-006, Revision 1. The applicable sections of the FSAR and TSR are listed below along with the requirements for the PIC skid controls. 
RPP-5492

REVISION 0

\subsection{FSAR REQUIREMENTS}

FSAR chapter 2, Section 2.5.2, Waste Transfer identifies the following for PIC skid functional characteristics and controls and monitoring that impact the software program for the PIC skid PLC.

2.2.1 Section 2.5.2.1 (lines 33 and 34) states the salt well pump is allowed to run in an automatic mode.

2.2.2 Section 2.5.2.1.3 (lines 4 and 5) states the JR-1 valve has position indication that is monitored by the control system.

2.2.3 Section 2.5.2.1.4 has the following requirements for instrumentation and control.

PUMP DISCHARGE PRESSURE SENSORS: One discharge header pressure sensor provides a signal to the PLC for monitoring low and high pressures. A low or high pressure determined by the PLC will cause an alarm and pump shutdown. (See USQ evaluation TF-99-0830, rev. 0)

FLUSH WATER SUPPLY PRESSURE DETECTION SYSTEM: A pressure switch on the flush water line is activated upon change in pressure in the supply line, which results in an alarm and pump trip.

PROCESS FLOW TRANSMITTER AND TOTALIZER: A flow totalizer displays flow rates and totals gallons pumped.

\section{PUMP SUCTION AND DISCHARGE PRESSURE}

TRANSMITTERS: Pump suction and discharge pressure transmitters provide pressure readings. The transmitters are connected to the control systems and can be used as a backup to the pressure switches for pressure interlocks.

2.2.4 Section 2.5.2.1.5 states that salt well pumps on different tanks pumping into a common line are interlocked with each other. The control is to provide a safe and orderly shutdown of the group of pumps in the event a single pump shuts down.

2.2.5 Section 2.5.2.1.5.5 states that leak detectors connected to the skid control system are interlocked such that upon detection of a leak, a visual alarm and pump shutdown causing an audible alarm will occur. 


\section{RPP-5492 \\ REVISION 0}

2.2.6 Section 2.5.2.1.5.6 requires flammable gas monitoring and automatic interlock with the pump controls to shut down the pump upon reaching of $25 \%$ of the lower flammability limit (LFL) in the pump pit.

\subsection{COMPLIANCE WITH FSAR FUNCTIONAL REQUIREMENTS AND CONTROLS}

\subsubsection{Software design requirements must meet the functional} requirements and controls stated above. The paragraphs below describe how these are met.

\subsubsection{Salt well pump automatic mode:}

An Auto/Manual selection is provided on the DTAM, which interfaces, with the PLC ladder logic.

\subsubsection{JR-1 valve position indication:}

Transducers LS-1 and LS-2 input to the PLC. The PLC logic interprets these inputs and the DTAM displays the JR-1 valve position as "PROCESS" or as "NON-PROCESS" when JR-1 is in the flush or prime positions.

\subsubsection{Low discharge pressure causes alarm and pump shutdown:}

Pressure sensor SALW-PT-6015N inputs to the PLC. The PLC processes an alarm for pressures below 15psig or above 140psig. The alarm signal goes to the DTAM and strobe light. A lowpressure condition opens the pump motor control interlock circuit after a 30 second delay causing the pump to shutdown. The highpressure condition opens the pump motor control interlock circuit after a 3 second delay causing the pump to shutdown.

\subsubsection{A pressure transducer on the flush water line causes alarm and pump shutdown:}

Pressure transducer SALW-PT-6014N inputs to the PLC. The PLC processes an alarm for pressure in the jumper flush line. The alarm must actuate before the pressure reaches $18 \mathrm{psig}$. The alarm signal goes to the DTAM and strobe light. The high flush pressure opens the pump motor control interlock circuit after a 3 second delay causing the pump to shutdown. 


\section{REVISION 0}

\subsubsection{A flow totalizer displays flow rate and total gallons pumped:}

A flow head measures the flow in the pump jumper and sends the information to a flow converter inside the Instrument Enclosure. The flow converter sends a 4 to $20 \mathrm{~mA}$ signal to the PLC. The PLC processes the signal and the flow rate and total gallons are displayed on the DTAM.

2.3.1.6 Pump suction and discharge pressures are displayed: Signals from transducers are sent to digital display units in the Instrument Enclosure. The discharge pressure signal is also processed by the PLC and displayed on the DTAM.

\subsubsection{Salt well pumps on different tanks that pump into a} common line are interlocked with each other:

All eight U-farm tanks will pump into a common line going to tank SY-102. The following interlocks will be set up between the skids.

2.3.1.7.1 A 30-minute timer starts when any skid is shutdown and has JR-1 not in the prime position and. Upon timing out, the other skids will shutdown.

2.3.1.7.2 High jumper flush pressure and high recirculation pressure on any skid starts a 20-minute timer and shuts down the other skids upon timing out.

2.3.1.7.3 Skids with JR-1 in flush position; leak detection; and leak detector trouble, loss of recirculation flush signal, or loss of jumper flush signal will cause pump shutdowns after a 3-second delay.

\subsubsection{Leak detectors connected to the skid are interlocked to} cause visible and audible alarms upon leak detection: Leak detector relays provide discrete inputs to the PLC. Upon detection of a leak or leak detector trouble, alarms and pump shutdown occur after a 3 second delay. For U-109, leak detection at the skid monitors the U-109 distribution pit, pump pit and the Over-ground transfer line that runs between these two pits. 
RPP-5492

REVISION 0

2.3.1.10 Flammable gas monitoring and automatic interlock to shut down pump upon reaching $25 \%$ of LFL.

The Combustible gas monitor (CGM) provides a discrete input to the PLC. Upon reaching $20 \%$ of the LFL, the PLC immediately shuts down the pump. The PLC also removes power from the pump and jumper heat trace cables by deenergizing the heat trace relays in the Instrument Enclosure if the heat trace is ON or prevents the heat trace relays from energizing if the heat trace is OFF. A CGM trouble alarm is also monitored by the PLC and shuts down the pump after a 3-second delay when the trouble contact opens.

2.3.1.11 There are other conditions that will cause an individual skid to shutdown. These conditions are communications failure with the software, signal loss from the jumper flush and recirculation flush transducers, and low flow in the recirculation line. There are other conditions monitored by all the skids on a common line causing pump shut downs. These conditions for U-farm are leaks or detector troubles at Leak Detector station 4 (valve pits U-C, U-D, and $\mathrm{COB} 30$ ) and Leak Detector station 5 (valve pits U-A, U-B, and $C O B$ 33); no dilution water flow; leaks or trouble at the SY-B valve pit; and if the portable exhauster is on line. All of these conditions for individual and all the skids are tied into the interlock circuit in the PLC ladder logic for the pump permissive. 


\section{REVISION 0}

\subsection{TSR REQUIREMENTS}

2.4.1 The software program meets the following Technical Safety Requirements. A description of how the program meets the requirements is explained.

LCO 3.1.2: Service Water Pressure Detection System. The recirc flush pressure and jumper flush pressure signals from the transducers are fed to the PLC. Upon the jumper flush line pressure reaching $15 \mathrm{psig}$, an alarm activates and the jet pump shuts down. Upon the recirculation flush line pressure reaching $15 \mathrm{psig}$, an alarm activates and the jet pump shuts down.

LCO 3.1.3: Transfer Leak Detection Systems. The primary leak detector for the pump pit is monitored for leaks and/or trouble with the signals being sent to the PLC. The PLC logic activates an alarm and shuts down the jet pump upon receiving leak or trouble signals from the leak detection systems. Leak detection signals from other leak detection systems along the transfer route are also fed to the PLC and cause a jet pump shut down.

Administrative Control 5.10.2.c.6: Ignition Controls, Ignition Source Control Set \#2. The jet pump does not meet Class I, Division 2, Group B design. Therefore, the jet pump must be automatically shut down upon detection of combustible gas in the pump pit. The Combustible Gas Monitor (CGM) monitors the pump pit for combustible gas. Contacts in the CGM transmitter open upon sensing greater than $20 \%$ of the Lower Flammability Limit (LFL) or CGM trouble. The PLC monitors the CGM contacts. Upon detecting opening of the CGM contacts for high LFL or trouble, the jet pump will shut down and power is removed from the pump and jumper heat trace. 
RPP-5492

REVISION 0

\subsection{SOFTWARE DESCRIPTION}

\subsection{U-109 SOFTWARE}

The software programs for the PLC and DTAM at U-109 are based upon the program for $U-102$. Software changes made specifically for $U-109$ are identified below.

3.1.1 Temperature in the Water and WFIE cabinets is monitored by the PLC. An alarm occurs when the temperature equals or less than 35 degrees $F$. The alarm resets at 40 degrees $F$.

3.1.2 The CGM signal display at the DTAM has a range of 0 to $100 \%$ instead of 0 to $30 \%$.

3.1.3 The U-109 program incorporates changes made to the other $U$ farm skids at $U-103, U-105$ and $U-102$. These changes include a minimum dilution flow rate and an increase in the communications inputs from other skids over the $\mathrm{DH}-485$ network.

\subsection{PLC INPUTS/OUTPUTS}

3.2.1 Outputs:

3.2.1.1 Current to Pneumatic (I/P) converter control consisting of a 4 to $20 \mathrm{~mA}$ signal to control airflow to the DOV.

3.2.1.2 Dilution metering pump control consisting of a 4 to $20 \mathrm{~mA}$ signal.

3.2.1.3 Motor starter permissive is $120 \mathrm{vac}$ power to the jet pump motor contactor to allow the pump to start.

3.2.1.4 A 120vac-output power signal is sent as appropriate to the four indicator lights (red, green, blue and amber) on the instrument panel.

3.2.1.5 A 120vac-output power to the pump and jumper heat trace relays.

3.2.1.6 An 120vac-output signal to the dilution-metering pump control circuit.

3.2.1.7 An 120vac-output signal to the dilution contactor.

3.2.1.8 A 120vac output power to the strobe when alarms occur.

3.2.1.9 A 120vac-output power to the horn when the jet pump shuts down. 
RPP-5492

REVISION 0

3.2.1.10 Output to the $\mathrm{DH}-485$ line as to skid status for JR-1 prime and flush positions, leak detection alarms, skid shutdown, loss of recirculation and/or jumper flush signals, and high recirculation flush or high jumper flush pressures.

\subsubsection{Inputs:}

3.2.2.1 Combustible gas monitor flammability levels are received on a 4 to $20 \mathrm{~mA}$ signal representing a 0 to 100 percent range of the lower flammability limit.

3.2.2.2 Discharge pressure signal of 4 to $20 \mathrm{~mA}$ from transducer on jumper representing 0 to 300 -psig.

3.2.2.3 Specific gravity signal of 4 to $20 \mathrm{~mA}$ from transmitter in WFIE cabinet representing 0 to 20 inches.

3.2.2.4 Weight factor signal of 4 to $20 \mathrm{~mA}$ from transmitter in WFIE cabinet representing 0 to 500 inches.

3.2.2.5 Water tank level signal of 4 to $20 \mathrm{~mA}$ from transmitter in Water cabinet representing 0 to 62 inches.

3.2.2.6 Flow converter signal of 4 to $20 \mathrm{~mA}$ from transmitter in Instrument cabinet representing 0 to $8 \mathrm{gpm}$.

3.2.2.7 Recirculation flush pressure signal of 4 to $20 \mathrm{~mA}$ from transducer on flush line representing 0 to $30 \mathrm{psig}$.

3.2.2.8 Flush line pressure from transducer (old PS-2) of 4 to $20 \mathrm{~mA}$ representing 0 to 30 psig.

3.2.2.9 Discrete input from dilution system. Contact opens on no dilution water flow to provide alarm.

3.2.2.10 Discrete input from Combustible Gas Monitor (CGM) instruments. Two inputs are received. One for flammable gas levels greater than $20 \%$ of the LFL and one for CGM trouble. Either will cause an alarm, jet pump shutdown and the deenergizing of the pump and jumper heat trace relays.

3.2.2.11 Discrete inputs from the leak detection relays. Contacts from the MTL2313A relay open upon leak detection circuit trouble to cause an alarm and jet pump shutdown. Contacts from the 5300 relay open upon detection of a leak to cause an alarm and jet pump shutdown.

3.2.2.12 Discrete input from the recirculation flow meter located in the Water Cabinet. Contact opens upon low flow in the recirculation line to cause an alarm and jet pump shut down.

3.2.2.13 Discrete inputs from transducers LS-1 and LS-2 located on the pump assembly. These transducers monitor the position of the JR-1 three-way valve. The inputs feed into displays, alarms and/or pump permissive inputs. 
RPP-5492

REVISION 0

3.2.2.14 A 4 to $20 \mathrm{~mA}$ signal from the transfer line pressure transducer. The PLC uses this signal to monitor for low and high pressures on the transfer line.

3.2.2.15 Discrete input MR-1 from the jet pump motor starter. This contact closes when the motor contactor is energized.

3.2.2.16 Thermocouple inputs from the PLC enclosure, air compressor enclosure, water cabinet, WFIE cabinet, jet pump motor bearing, and pump jumper assembly.

3.2.2.17 Input from the $\mathrm{DH}-485$ line as to the status of other skids and leak detector stations along the waste line transfer path. This input is used for alarms and jet pump shutdown when appropriate.

\subsection{DTAM DISPLAYS, DATA ENTRY AND ALARMS}

\subsubsection{DTAM DISPLAYS}

3.3.1.1 Alarm signals from the PLC.

3.3.1.2 Analog data inputs from the PLC for specific gravity, discharge pressure, recirculation flush pressure, jumper flush pressure, flammable gas levels, Diaphragm Operated Valve (DOV) parameters, and dilution ratio.

3.3.1.3 Temperatures for the Instrument Enclosure, the Air compressor cabinet, WFIE cabinet, Water cabinet, Pump motor, and jumper assembly

\subsubsection{DTAM DATA ENTRY}

3.3.2.1 Signal to turn jumper and pump heat trace ON or OFF.

3.3.2.2 Signal to set pump operation to Manual or Automatic.

3.3.2.3 Alarm acknowledge signal.

3.3.2.4 DOV set point and parameters.

3.3.2.5 Pump ON or OFF input.

3.3.2.6 Jumper temperature setpoint.

3.3.2.7 Dilution ratio setpoint input.

3.3.2.8 Dilution minimum flow input.

\subsubsection{DTAM ALARMS}

3.3.3.1 Individual alarm screens for each incoming alarm.

3.3.3.2 Screens 101 to 153 for individual alarm acknowledge. 


\subsection{JET PUMP INTERLOCKS}

There are two jet pump interlock strings in the ladder diagram. One string is for equipment along the transfer path that monitors or feed into the transfer route. The other interlock string consists of the local PIC skid conditions. These two interlock strings are identified below for the U-109 ladder logic. All the interlock conditions must be satisfied for the jet pump to run or if the pump is running and a condition becomes unsatisfied, the pump is shut down.

3.4.1 Interlock string for other equipment along the transfer route:

3.4.1.1 COB U-33 leak

3.4.1.2 COB U-33 trouble

3.4.1.3 Dilution tank no flow

3.4.1.4 Leak detected by any of the seven other U-farm PIC skids

3.4.1.5 Any of the seven other U-farm PIC skids with JR-1 in flush position

3.4.1.6 Leak detector station \#4 trouble

3.4.1.7 The 30 minute time out by any of the other seven U-farm PIC skids being shutdown and JR-1 not in the prime position

3.4.1.8 Valve pit U-C leak

3.4.1.9 Valve pit U-C trouble

3.4.1.10 Valve pit U-D leak

3.4.1.11 Valve pit U-D trouble

3.4.1.12 Any of the other seven U-farm PIC skids with high jumper flush pressure

3.4.1.13 Leak station \#5 trouble

3.4.1.14 Valve pit U-A leak

3.4.1.15 Valve pit U-A trouble

3.4.1.16 Valve pit U-B leak

3.4.1.17 Valve pit U-B trouble

3.4.1.18 COB U-30 leak

3.4.1.19 COB U-30 trouble

3.4.1.20 Any of the other seven U-farm PIC skids with high recirculation line pressure

3.4.1.21 Any of the other seven U-farm PIC skids with loss of recirculation flush pressure signal

3.4.1.22 Any of the other seven U-farm PIC skids with loss of jumper flush pressure signal

3.4.1.23 Portable exhauster on line

3.4.1.24 Leak or trouble alarm from SY-02A and SY-B valve pits 


\section{RPP-5492 \\ REVISION 0}

3.4.2 Interlock string monitoring the U-109 PIC skid conditions:

3.4.2.1 Low pressure from the PXPT transducer while pump is running

3.4.2.2 High pressure from the PXPT transducer

3.4.2.3 High jumper flush pressure

3.4.2.4 JR-1 in the flush or prime position

3.4.2.5 Pump pit leak

3.4.2.6 Data communications failure on the $\mathrm{DH}-485$ link

3.4.2.7 Recirculation line low flow

3.4.2.8 FGM greater than $25 \%$ of LFL (Not used, replaced by CGM interlocks.)

3.4.2.9 Jumper flush pressure signal loss

3.4.2.10 Pump pit leak detector trouble

3.4.2.11 Recirculation line high flush pressure

3.4.2.12 Leak detector 1 trouble

3.4.2.13 Recirculation flush pressure signal loss

3.4.2.14 Leak detector 1 leak

3.4.2.15 CGM greater than $20 \%$ of LFL

3.4.2.16 CGM trouble

3.4.2.17 Other equipment interlock (the above string) 
RPP-5492

REVISION 0

\subsection{INTERLOCK and ALARM CONDITIONS}

Alarms occur at the PIC skid when the above interlocks are not satisfied. Some alarms are immediate while some are delayed. The alarm conditions are listed below along with the response time for the alarm to occur and whether the jet pump shuts down.

\begin{tabular}{|c|c|c|}
\hline CONDITION & TIME DELAY & PUMP SHUTDOWN \\
\hline Low transfer pressure & 30 seconds & Yes \\
\hline Recirc low flow & 30 seconds & Yes \\
\hline Communications failure & 60 seconds & Yes \\
\hline High flush press on other skids & 20 minutes & Yes \\
\hline $\begin{array}{l}\text { Other skids shutdown and in JR-1 } \\
\text { not in prime position }\end{array}$ & $\begin{array}{l}30 \text { minute, then } 3 \\
\text { second delay }\end{array}$ & Yes \\
\hline JR-1 in flush \# or prime ${ }^{*}$ & None & Yes \\
\hline Pump shutdown* & None & $\cdots$ \\
\hline FGM greater than $25 \%$ LFL & None(not used) & Yes(not used) \\
\hline High transfer pressure & 3 seconds & Yes \\
\hline $\begin{array}{l}\text { Jumper flush pressure greater than } \\
15 \text {-psig \# }\end{array}$ & 3 seconds & Yes \\
\hline Pump trouble & 3 seconds & Yes \\
\hline Distribution Pit leak \# & 3 seconds & Yes \\
\hline $\begin{array}{c}\text { Distribution Pit leak detector } \\
\text { trouble }\end{array}$ & 3 seconds & Yes \\
\hline $\begin{array}{l}\text { Leak detector } 1 \text { leak in (Pump pit } \\
\text { and OGT) }\end{array}$ & 3 seconds & Yes \\
\hline $\begin{array}{l}\text { Leak detector } 1 \text { trouble (Pump pit } \\
\text { and OGT) }\end{array}$ & 3 seconds & Yes \\
\hline Recirc flush pressure signal loss \# & 3 seconds & Yes \\
\hline $\begin{array}{l}\text { Another U-farm skid with JR-1 in } \\
\text { flush position }\end{array}$ & 3 seconds & Yes \\
\hline Jumper flush pressure signal loss \# & 3 seconds & Yes \\
\hline $\begin{array}{l}\text { Another U-farm skid with leak } \\
\text { alarms }\end{array}$ & 3 seconds & Yes \\
\hline $\begin{array}{l}\text { Another U-farm skid with loss of } \\
\text { jumper flush pressure signal }\end{array}$ & 3 seconds & Yes \\
\hline $\begin{array}{l}\text { Another U-farm skid with loss of } \\
\text { recirculation flush pressure signal. }\end{array}$ & 3 seconds & Yes \\
\hline Leak Station 4 trouble & 3 seconds & Yes \\
\hline Leak Station 5 trouble & 3 seconds & Yes \\
\hline Valve Pit U-A Leak & 3 seconds & Yes \\
\hline Valve Pit U-A trouble & 3 seconds & Yes \\
\hline Valve Pit U-B Leak & 3 seconds & Yes \\
\hline Valve Pit U-B trouble & 3 seconds & Yes \\
\hline
\end{tabular}


RPP-5492

REVISION 0

\begin{tabular}{|c|c|c|}
\hline CONDITION & TIME DELAY & PUMP SHUTDOWN \\
\hline Valve Pit U-C Leak & 3 seconds & Yes \\
\hline Valve Pit U-C trouble & 3 seconds & Yes \\
\hline Valve Pit U-D Leak & 3 seconds & Yes \\
\hline Valve Pit U-D trouble & 3 seconds & Yes \\
\hline COB U-30 Leak & 3 seconds & Yes \\
\hline COB U-30 Trouble & 3 seconds & Yes \\
\hline COB U-33 Leak & 3 seconds & Yes \\
\hline COB U-33 Trouble & 3 seconds & Yes \\
\hline $\begin{array}{c}\text { Valve Pits SY-A or SY-B or SY- } \\
\text { 02A pump pit alarm }\end{array}$ & 3 seconds & Yes \\
\hline $\begin{array}{l}\text { Another U-farm skid with high } \\
\text { recirc pressure }\end{array}$ & 20 minutes & Yes \\
\hline Dilution tank no flow & 5 minutes & Yes \\
\hline $\begin{array}{l}\text { Recirc pressure greater than } \\
\text { 15PSIG \# }\end{array}$ & 3 seconds & Yes \\
\hline $\begin{array}{c}\text { Portable Exhauster on line } \\
\text { (exhauster not physically installed) }\end{array}$ & 3 seconds & Yes \\
\hline CGM high LFL & None & Yes \\
\hline CGM trouble & 3 seconds & Yes \\
\hline $\begin{array}{c}\text { Pump is less than } 40 \text { degrees } F \text { or } \\
\text { the jumper is greater than } 225 \\
\text { degrees } F\end{array}$ & 3 seconds & No \\
\hline Pump trouble & 3 seconds & No \\
\hline Water tank low level & None & No \\
\hline $\begin{array}{l}\text { Instrument enclosure greater than } \\
130 \text { degrees } \mathrm{F} \\
\end{array}$ & None & No \\
\hline $\begin{array}{c}\text { Air compressor enclosure greater } \\
\text { than } 130 \text { degrees } F\end{array}$ & None & No \\
\hline $\begin{array}{l}\text { Water cabinet less than } 35 \\
\text { degrees } \mathrm{F}\end{array}$ & None & No \\
\hline $\begin{array}{c}\text { WFIE cabinet less than } 35 \text { degrees } \\
\text { F }\end{array}$ & None & No \\
\hline Salt well low level & None & No. DOV closes \\
\hline
\end{tabular}

* If the U-109 skid "N" shuts down and the JR-1 valve is not in the prime position, a 30minute timer starts. Upon timing out, any other skids on the same transfer route will shut down.

\# These alarms will also cause any other skids on the same transfer route to shut down. 


\subsection{PIC SKID COMMUNICATIONS}

The PIC skid at U-109 communicates with the computer at 271-U over a DH-485 link. The PLC monitors the link to verify the communications link is functional. This communications link monitoring ties into the interlock circuit for the jet pump control.

PIC skid status information is sent over the link to the $271-U$ computer. Skid status information monitored is listed below.

3.6.1 JR-1 in prime position

3.6.2 JR-1 in flush position

3.6.3 PIC skid shut down

3.6.4 Leak detected or leak detector trouble in pump pit

3.6.5 Skid high flush pressure

3.6.6 Skid high recirc flush pressure

3.6.7 RFPT signal loss

3.6.8 JFPT signal loss

When other PIC skids are installed in U-farm, the above information will affect those skid's operation. Other skids connected in U-farm will shut down either immediately or after some delay should the $U-109$ skid be shut down conditions 3.6.2, or 3.6.4 through 3.6.8 occurring. Should the U-109 skid shut down and JR-1 is not placed in the prime position within 30 minutes, then other skids connected in U-farm will shut down. A similar type of condition will exist for the skid at U-109 which will shutdown upon shut down of other U-farm skids.

The software is written such if other skids are not connected, no alarm or shut down conditions exist. Fault conditions from other U-farm skids are provided to the U-109 skid over the $\mathrm{DH}-485$ link. As explained in the interlock section above, a loss of the DH- 485 communications causes jet pump to shut down. There is also communications between the U-farm OCS and the S/SX-farm OCS computers. This communication is used to send information concerning leak detection alarm or trouble in the SY-02A pump pit and the SY-B valve pit. 


\subsection{SOFTWARE PROGRAM DESCRIPTION}

This section explains the input and output requirements of the PLC; alarm set points and control system functions. These requirements are used to develop the ladder logic for pump control and alarm actuation. The complete ladder logic is in Appendix B for the PLC and Appendix C for the DTAM program.

\subsection{Program locations}

The program for the PLC is stored in the CPU located in the PIC skid Instrument Enclosure. The DTAM program is stored in the DTAM unit located on the door of the Instrument Enclosure. The programs for the PLC and DTAM are developed from software on a laptop computer. After the program is written on the laptop, it is downloaded to the PLC and DTAM through an interface link.

Backup copies of the PLC and DTAM programs are stored on 3-1/2" disks. There are two backup copies, one for the design agent and one for file. The backup copies represent the final program in the PLC and DTAM. The PLC file is "U109NEW1R1" and the DTAM file is "U109NEW1."

\subsection{Program layout}

4.2.1 The program is written where ladder 2 is the main program. Ladders 3 through 20 are subroutines. The main program uses the subroutines as required during running of the program. The subroutines are listed below as to what function each performs.

4.2.2 Subroutine 3: Program initial conditions are set including initializing the thermocouple module to receive inputs.

4.2.3 Subroutine 4: Inputs to the PLC modules are gathered and placed into an $\mathrm{N} 60:^{*} /{ }^{*}$ address location. The rest of the program when needing that particular input uses this address.

4.2.4 Subroutine 5: Checks for alarm conditions and has the logic for the main interlock for jet pump control.

4.2.5 Subroutine 6: Contains the logic for the interlock logic for other equipment connected to the transfer route.

4.2.6 Subroutine 7: Contains the logic for the jet pump and dilution pump controls to run these pumps. The logic for the total flow calculation to be displayed on the DTAM is this subroutine. 
RPP-5492

REVISION 0

4.2.7 Subroutine 8: This subroutine is the master alarm acknowledge logic.

4.2.8 Subroutine 9: Heat trace control logic

4.2.9 Subroutines 10 and 14: These subroutines are for the $\mathrm{DH}-485$ communications link.

4.2.10 Subroutine 11: This subroutine is only used if the jet and dilution pumps are to be run by control from the $271-\mathrm{U}$ computer. (This is not used nor was this function tested.)

4.2.11 Subroutine 12: This subroutine handles the inputs for the other equipment status on the transfer route.

4.2.12 Subroutines 13 and 16: Flow meter output routines.

4.2.13 Subroutine 15: This subroutine is the 30-minute time for the other skids in U-farm when a skid is shut down and in the process position. When the 30-minute timer times out, the skid shuts down unless JR-1 of the shut down skid is placed in the prime position.

4.2.14 Subroutine 17: Individual alarm acknowledge routine.

4.2.15 Subroutine 18: Alarm output to the strobe light, horn and/or instrument panel amber, blue, green or red indicator lights.

4.2.16 Subroutine 19: Sends outputs to the PLC output modules.

4.2.17 Subroutine 20: Sends an output to the dilution metering pump controls.

\subsection{Inputs and Outputs}

Inputs are brought into the PLC through modules 1 through 12. For U-109 the inputs are assigned addresses N60:*. Appendix $A$ lists the addressing for the inputs to the PLC. Outputs are also assigned an N60:* address and can be found in Appendix A.

\subsection{Temperature}

The jumper and pump temperatures are to remain between 40 degrees $F$ and 225 degrees $F$ when the heat trace is $O N$. An alarm actuates for temperatures outside these limits. The temperature is monitored by the PLC through thermocouple probes at the pump and jumper assemblies. 


\section{REVISION 0}

The temperature of the Instrument Enclosure is not to exceed 130 degrees $F$. An alarm actuates for temperatures equal to or greater than 130 degrees $F$. The alarm resets when the temperature drops below 125 degrees $F$.

The temperature of the Air Compressor Enclosure is not to exceed 130 degrees $F$. An alarm actuates for temperatures equal to or greater than 130 degrees $F$. The alarm resets when the temperature drops below 125 degrees $F$.

The temperature of the WFIE Cabinet is not to decrease below 35 degrees $F$. An alarm actuates for temperatures less than 35 degrees $F$. The alarm resets when the temperature rises above $\mathbf{4 0}$ degrees $F$.

The temperature of the Water Cabinet is not to decrease below 35 degrees $F$. An alarm actuates for temperatures less than 35 degrees $F$. The alarm resets when the temperature rises above 40 degrees $F$.

\subsection{Alarms}

Various inputs to the PLC provide information to determine if an alarm condition exists. Alarm conditions are processed in subroutine 5 in the PLC ladder logic program. Addresses N20:15/ ${ }^{\star}, \mathrm{N} 20: 16 /{ }^{*}, \mathrm{~N} 20 ; 55 /^{*}$ and $\mathrm{N} 20: 56 /^{*}$ are actuated upon alarm conditions and turn on the strobe light (subroutine 18 of the PLC ladder logic) at the Instrument Enclosure. The horn is turned on when a pump shutdown occurs. Addresses N20:24/ ${ }^{*}, \mathrm{~N} 20: 25 /^{*}, \mathrm{~N} 20: 51 /^{*}$ and N20:52/ ${ }^{*}$ provide alarm input to the DTAM.

Alarms are acknowledged at the DTAM on screen \#23. Acknowledging the alarm clears the signal to the strobe and horn only (subroutine 17 of the PLC ladder logic). The visual alarm remains on the DTAM screen until the alarm condition clears in the field.

Appendix $\mathrm{A}$ lists all the alarm conditions monitored, the addresses and alarm messages. Appendix $C$ is a copy of the DTAM program containing all the alarm screens.

\subsection{Pump Control}

Subroutine 7 outputs to the motor starter for the jet pump. Three conditions in the software logic must be satisfied to provide the signal for the motor contactor to close. These are: 1) the interlocks must be satisfied. These interlocks include the skid interlocks in subroutine 5 and interlocks to any other tanks on the same transfer line as required in subroutine 6. 2) Pump to the ON position from the DTAM and 3) pump trouble cleared in subroutine 5. Pump trouble clears when the pump is ON from the DTAM and the auxiliary contact at the motor starter is closed. 


\subsection{Program Set Points}

The set points for U-109 are the same as U-102. Each set points is identified below along with the PLC logic values.

4.7.1 Jumper flush pressure alarm point: The jumper flush pressure is set to alarm at pressures greater than 15PSI. This is to meet the requirements of LCO 3.1.2 that requires the service water pressure to be less than 20psi. The pressure transducer range is 0 to $30 \mathrm{psi}$, with a 4 to $20 \mathrm{~mA}$ output signal to the PLC. The internal logic value of the PLC for a 4 to $20 \mathrm{~mA}$ signal is 3277 to 16384 . The PLC logic value for an alarm set point of $15 \mathrm{psi}$ is 9830 .

4.7.2 Recirculation flush pressure set point: The recirculation flush pressure is set to alarm at $15 \mathrm{psi}$. This set point is to meet the same requirements as the jumper flush pressure, but is set to detect any leakage past the check valves in the recirculation line during pumping of the waste. The transducer range is 0 to $30 p s i$, with a 4 to $20 \mathrm{~mA}$ signal output to the PLC. The PLC logic value for $15 \mathrm{psi}$ is 9830 .

4.7.3 Jumper and recirculation flush pressure signal loss: The input signal from the jumper and recirculation flush pressure transducers is not fail-safe. Therefore, the 4 to $20 \mathrm{~mA}$ signal is monitored to a signal loss due to a failed transducer, broken wire or failure of the intrinsic safe module. The PLC logic value is set at 2500 . This value is selected because the steady state value of the transducer is below the 3277 when the piping has no liquid inside. The 2500 value will prevent nuance alarms and pump shutdowns.

4.7.4 Water tank level: PIC skid "N" has a 62-inch high water tank. The set points for low water level and reset were calculated to correspond to the relative volume as in the older skids with a 50inch high tank. The alarm level is set at 12.25 inches and the reset level is set at 15.5 inches. The level transmitter outputs a 4 to $20 \mathrm{~mA}$ signal to the PLC. The corresponding PLC logic value for 12.25 inches is 5867 and for 15.5 inches is 6554 .

4.7.5 Low and High pressure: a pressure transducer monitors the pressure on the pump and jumper assembly transfer line. A 4 to $20 \mathrm{~mA}$ signal is sent to the PLC. The PLC program determines a low and a high pressure alarm point. The low pressure is set at $15 \mathrm{psig}$ and the high pressure is set at 140psig. The PLC logic value for $15 p s i g$ is 3932 and for 140psig is 9394 . 


\subsection{Other U-farm Skids}

Other U-farm skids when installed will interface over the $\mathrm{DH}-485$ data link to the U-109 skid "N". The PLC ladder logic is set up for these future interfaces. Future skids in U-farm will be at tanks U-106, U-107, U-108, U111 , and the emergency skid. The types of interfaces are identified below. The PLC logic is an open contact, which is monitored for a closed condition causing a timer to start and/or the skid to shut down.

4.8.1 Skid JR-1 alarm conditions: When another skid has JR-1 in the flush position, the U-109 skid will shut down after 3 second delay upon receiving the signal over the $\mathrm{DH}-485$ link. If another skid has JR-1 in the process position and that skid is in the shut down mode, a 30-minute timer starts. Upon timing out of the timer, the U-109 skid will shut down after a 3-second delay. The 30-minute timer allows operations personnel time to move the JR-1 to the prime position upon skid shut down.

4.8.2 High flush pressures: Upon high flush pressure in the jumper of an on-line skid, a 20-minute timer starts. Timing out of the timer will cause the U-109 skid to shut down. The 20-minute timer allows operations personnel time to perform work on an on-line skid without immediately shutting down the other on-line skids. High recirculation pressure on a skid will cause the shut down of the other on-line skids after a 20-minute delay upon receiving the signal over the $\mathrm{DH}-485$ link.

4.8.3 Leak detection or leak detector trouble: Leak detection or trouble at Leak Detector Stations 4 and/or 5 in U-farm, at the SY-02A, SY-A or SY-B valve pits, and/or at any other on-line skid in U-farm, will cause the U-109 skid to shut down after a 3 second delay upon receiving the signal over the $\mathrm{DH}-485$ link.

4.8.4 Flush pressure signal losses: The loss of signal to the PLC from either the jumper flush pressure or the recirculation flush pressure transducers on any of the other seven U-farm skids, will cause the U-109 skid to shutdown after a 3-second delay upon receiving the signal over the $\mathrm{DH}-485$ link. 
RPP-5492

REVISION 0

\section{APPENDICES}

APPENDIX A: PLC AND DTAM ADDRESSING

APPENDIX B: PLC LADDER LOGIC PROGRAM

APPENDIX C: DTAM PROGRAM 


\section{RPP-5492 REVISION 0 \\ APPENDIX A}

PLC ADDRESSES

\begin{tabular}{|c|c|c|c|c|}
\hline TERMINATION & SIGNAL & TAG & ADDRESS & DESCRIPTION \\
\hline $0: 1.0$ & ANALOG OUTPUT & & N60:0 & \\
\hline $0: 1.1$ & ANALOG OUTPUT & & N60:1 & \\
\hline $1: 1.0$ & ANALOG INPUT & $\mathrm{CGM}$ & N60:2 & CGM ANALOG (PIT) \\
\hline $1: 1.1$ & ANALOG INPUT & FGM & N60:3 & FGM ANALOG (DOME)(RESERVED) \\
\hline $0: 2.0$ & ANALOG OUTPUT & EPC & N60:4 & OUTPUT TO DOV \\
\hline $0: 2.1$ & ANALOG OUTPUT & DIL & N60:5 & OUTPUT TO METERING PUMP \\
\hline $\mathrm{I}: 2.0$ & ANALOG INPUT & PDPT & N60:6 & PUMP DISCHARGE PRESSURE \\
\hline $1: 2.1$ & ANALOG INPUT & & N60:7 & \\
\hline $0: 3.0$ & ANALOG OUTPUT & & N60:8 & \\
\hline $0: 3.1$ & ANALOG OUTPUT & & N60:9 & \\
\hline $1: 3.0$ & ANALOG INPUT & & N60:10 & \\
\hline $1: 3.1$ & ANALOG INPUT & & N60:11 & \\
\hline $1: 4.0$ & $4-20 \mathrm{~mA}$ & SGT & N60:12 & SPECIFIC GRAVITY 0-20 IN. H2O \\
\hline $1: 4.1$ & $4-20 \mathrm{~mA}$ & WFT & N60:13 & WEIGHT FACTOR 0-500 IN. H2O \\
\hline $1: 4.2$ & $4-20 \mathrm{~mA}$ & LT & $\mathrm{N} 60: 14$ & WATER TANK LEVEL $0-62$ INCHES \\
\hline $1: 4.3$ & $4-20 \mathrm{~mA}$ & CNV & N60:15 & FLOW METER 0-8GPM \\
\hline $1: 5.0$ & $4-20 \mathrm{~mA}$ & RFPT & N60:16 & RECIRC FLUSH PRESSURE \\
\hline $1: 5.1$ & $4-20 \mathrm{~mA}$ & JFPT & N60:17 & JUMPER FLUSH PRESSURE (PS-2) \\
\hline $1: 5.2$ & $4-20 \mathrm{~mA}$ & PXPT & $\mathrm{N} 60: 18$ & TRANSFER PRESSURE (PS-1) \\
\hline $1: 5.3$ & & & N60:19 & \\
\hline $\mid: 6.0$ & & & N60:20 & $\ldots$ \\
\hline $\mid: 6.1$ & & 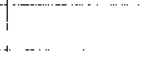 & $N 60: 21$ & \\
\hline $1: 6.2$ & & & N60:22 & \\
\hline $1: 6.3$ & & & N60:23 & \\
\hline $1: 7.0$ & $\mathrm{~T} / \mathrm{C}$ & TE-6002 & $\mathrm{N} 60: 24$ & PUMP TEMPERATURE \\
\hline $1: 7.1$ & T/C & TE- 6003 & N60:25 & JUMPER TEMPERATURE \\
\hline $1: 7.2$ & $\mathrm{~T} / \mathrm{C}$ & TE-6001 & N60:26 & AIR COMPR. CABINET TEMPERATURE \\
\hline $1: 7.3$ & $\mathrm{~T} / \mathrm{C}$ & TE-6004 & N60:27 & INSTRU. CABINET TEMPERATURE \\
\hline $0: 8.0$ & DISCRETE OUTPUT & & $N 60: 28 / 0$ & \\
\hline $0: 8.1$ & DISCRETE OUTPUT & DIL-P & $N 60: 28 / 1$ & DILUTION CONTACTOR \\
\hline $0: 8.2$ & DISCRETE OUTPUT & & $\mathrm{N} 60: 28 / 2$ & \\
\hline $0: 8.3$ & DISCRETE OUTPUT & DIL-C & N60:28/3 & DILUTION METERING PUMP \\
\hline $0: 8.4$ & DISCRETE OUTPUT & & N60:28/4 & \\
\hline $0: 8.5$ & DISCRETE OUTPUT & & $\mathrm{N} 60: 28 / 5$ & \\
\hline $1: 8.0$ & DISCRETE INPUT & & $N 60: 28 / 6$ & \\
\hline $1: 8.1$ & DISCRETE INPUT & DIL-F & $\mathrm{N} 60: 28 / 7$ & DILUTION TANK FLOW \\
\hline $1: 8.2$ & DISCRETE INPUT & CGM-AX & $N 60: 28 / 8$ & CGM HIGH LFL \\
\hline $1: 8.3$ & DISCRETE INPUT & FGM & N60:28/9 & FGM INTERLOCK(RESERVED) \\
\hline I:8.4 & DISCRETE INPUT & CGM-F & $N 60: 28 / 10$ & CGM FAULT \\
\hline $1: 8.5$ & DISCRETE INPUT & & $\mathrm{N} 60: 28 / 11$ & \\
\hline
\end{tabular}


RPP-5492 REVISION 0

APPENDIX A

\begin{tabular}{|c|c|c|c|c|}
\hline \multirow{2}{*}{\multicolumn{5}{|c|}{ ADDRESS }} \\
\hline & & & & \\
\hline $0: 9.0$ & DISCRETE OUTPUT & & N60:29/0 & \\
\hline $0: 9.1$ & DISCRETE OUTPUT & $\mathrm{DO2}$ & $N 60: 29 / 1$ & JUMPER HEAT TRACE ON \\
\hline $0: 9.2$ & DISCRETE OUTPUT & & $\mathrm{N} 60: 29 / 2$ & \\
\hline $0: 9.3$ & DISCRETE OUTPUT & DO3 & $N 60: 29 / 3$ & JUMPER HEAT TRACE HIGH SHUTOFF \\
\hline $0: 9.4$ & DISCRETE OUTPUT & & N60:29/4 & \\
\hline $0: 9.5$ & DISCRETE OUTPUT & & $N 60: 29 / 5$ & \\
\hline $1: 9.0$ & DISCRETE INPUT & LD3-2 & N60:29/6 & LEAK DETECTOR \#1 TROUBLE \\
\hline $1: 9.1$ & DISCRETE INPUT & LD1-1 & $\mathrm{N} 60: 29 / 7$ & PIT LEAK DETECTOR \\
\hline $1: 9.2$ & DISCRETE INPUT & & $N 60: 29 / 8$ & \\
\hline $1: 9.3$ & DISCRETE INPUT & LD3-1 & N60:29/9 & PIT LEAK DETECTOR TROUBLE \\
\hline $1: 9.4$ & DISCRETE INPUT & & N60:29/10 & \\
\hline $1: 9.5$ & DISCRETE INPUT & LD1-2 & $\mathrm{N} 60: 29 / 11$ & LEAK DETECTOR \#1 \\
\hline $0: 10.0$ & DISCRETE OUTPUT & & $\mathrm{N} 60: 30 / 0$ & \\
\hline $0: 10.1$ & DISCRETE OUTPUT & $B U Z Z$ & N60:30/1 & OUTPUT TO HORN \\
\hline $0: 10.2$ & DISCRETE OUTPUT & & N60:30/2 & \\
\hline $0: 10.3$ & DISCRETE OUTPUT & STRB & $\mathrm{N} 60: 30 / 3$ & OUTPUT TO STROBE LIGHT \\
\hline $0: 10.4$ & DISCRETE OUTPUT & & $\mathrm{N} 60: 30 / 4$ & \\
\hline $0: 10.5$ & DISCRETE OUTPUT & & $N 60: 30 / 5$ & \\
\hline |: 10.0 & DISCRETE INPUT & LS-1 & N60:30/6 & PROXIMITY LS-1 \\
\hline l:10.1 & DISCRETE INPUT & & $\mathrm{N} 60: 30 / 7$ & \\
\hline $\mathrm{l}: 10.2$ & DISCRETE INPUT & LS-2 & $\mathrm{N} 60: 30 / 8$ & PROXIMITY LS-2 \\
\hline $\mathrm{I}: 10.3$ & DISCRETE INPUT & & N60:30/9 & \\
\hline $\mathrm{I}: 10.4$ & DISCRETE INPUT & RECIRC & N60:30/10 & RECIRC. FLUSH PRESS. ALARM \\
\hline $1: 10.5$ & DISCRETE INPUT & & $\mathrm{N} 60: 30 / 11$ & \\
\hline $0: 11.0$ & DISCRETE OUTPUT & LGTR & $N 60: 31 / 0$ & OUTPUT TO RED LIGHT \\
\hline $0: 11.1$ & DISCRETE OUTPUT & LGTA & $\mathrm{N} 60: 31 / 1$ & OUTPUT TO AMBER LIGHT \\
\hline $0: 11.2$ & DISCRETE OUTPUT & MS-1 & N60:31/2 & PUMP MOTOR SWITCH ON \\
\hline $0: 11.3$ & DISCRETE OUTPUT & LGTB & $N 60: 31 / 3$ & OUTPUT TO BLUE LIGHT \\
\hline $0: 11.4$ & DISCRETE OUTPUT & & N60:31/4 & \\
\hline $0: 11.5$ & DISCRETE OUTPUT & LGTG & $N 60: 31 / 5$ & OUTPUT TO GREEN LIGHT \\
\hline $1: 11.0$ & DISCRETE INPUT & MR-1 & $N 60: 31 / 6$ & MOTOR POWER MONITOR \\
\hline $\mathrm{l}: 11.1$ & DISCRETE INPUT & & N60:31/7 & \\
\hline $\mathrm{I}: 11.2$ & DISCRETE INPUT & & $N 60: 31 / 8$ & \\
\hline |:11.3 & DISCRETE INPUT & & $N 60: 31 / 9$ & \\
\hline I:11.4 & DISCRETE INPUT & & $N 60: 31 / 10$ & \\
\hline $\mathrm{l}: 11.5$ & DISCRETE INPUT & & $N 60: 31 / 11$ & \\
\hline $\mathrm{I}: 12.0$ & $\mathrm{~T} / \mathrm{C}$ & & N60:32 & WATER CAB TEMP \\
\hline $\mid: 12.1$ & $\mathrm{~T} / \mathrm{C}$ & & N60:33 & WFIE TEMP \\
\hline $1: 12.2$ & $\mathrm{~T} / \mathrm{C}$ & & N60:34 & \\
\hline l:12.3 & $\mathrm{T} / \mathrm{C}$ & & N60:35 & \\
\hline
\end{tabular}




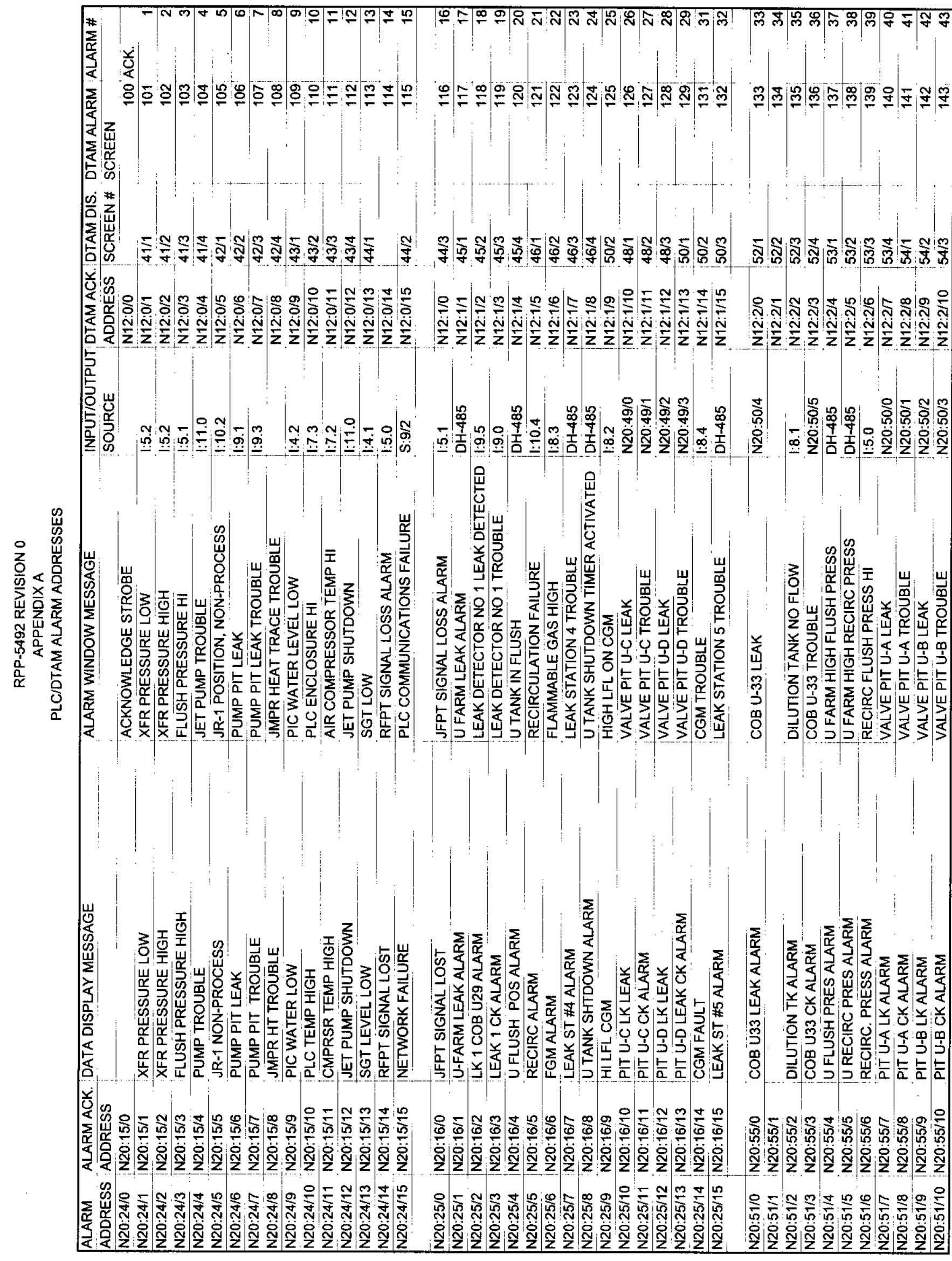




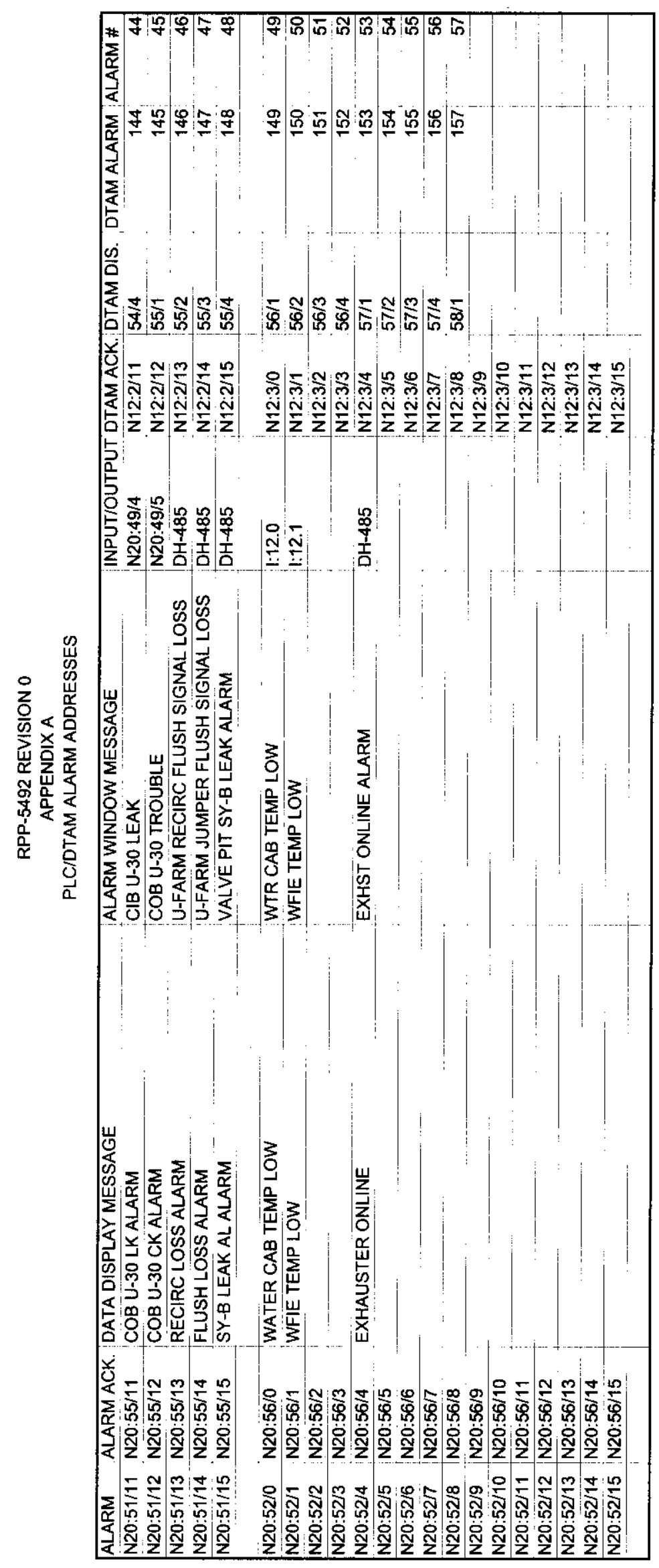


RPP-5492 REVISION 0 APPENDIX A

U-FARM NODE ADDRESSES

\begin{tabular}{|r|l|}
\hline NODE \# & LOCATION \\
0 & \\
1 & \\
2 & U-102, SKID M \\
3 & EMERG SKID \\
4 & $U-103$, SKID K \\
5 & $U-105$, SKID L \\
6 & $U-106$, SKID P \\
7 & $U-107$, SKID R \\
8 & $U-108$, SKID Q \\
9 & $U-109$, SKID N \\
10 U-111, SKID U \\
11 271-U, PLC \\
12 LEAK DET. STATION 4 \\
13 LEAK DET. STATION 5
\end{tabular}




\section{RPP-5492 REVISION 0 \\ APPENDIX A}

U-FARM ADDRESSES

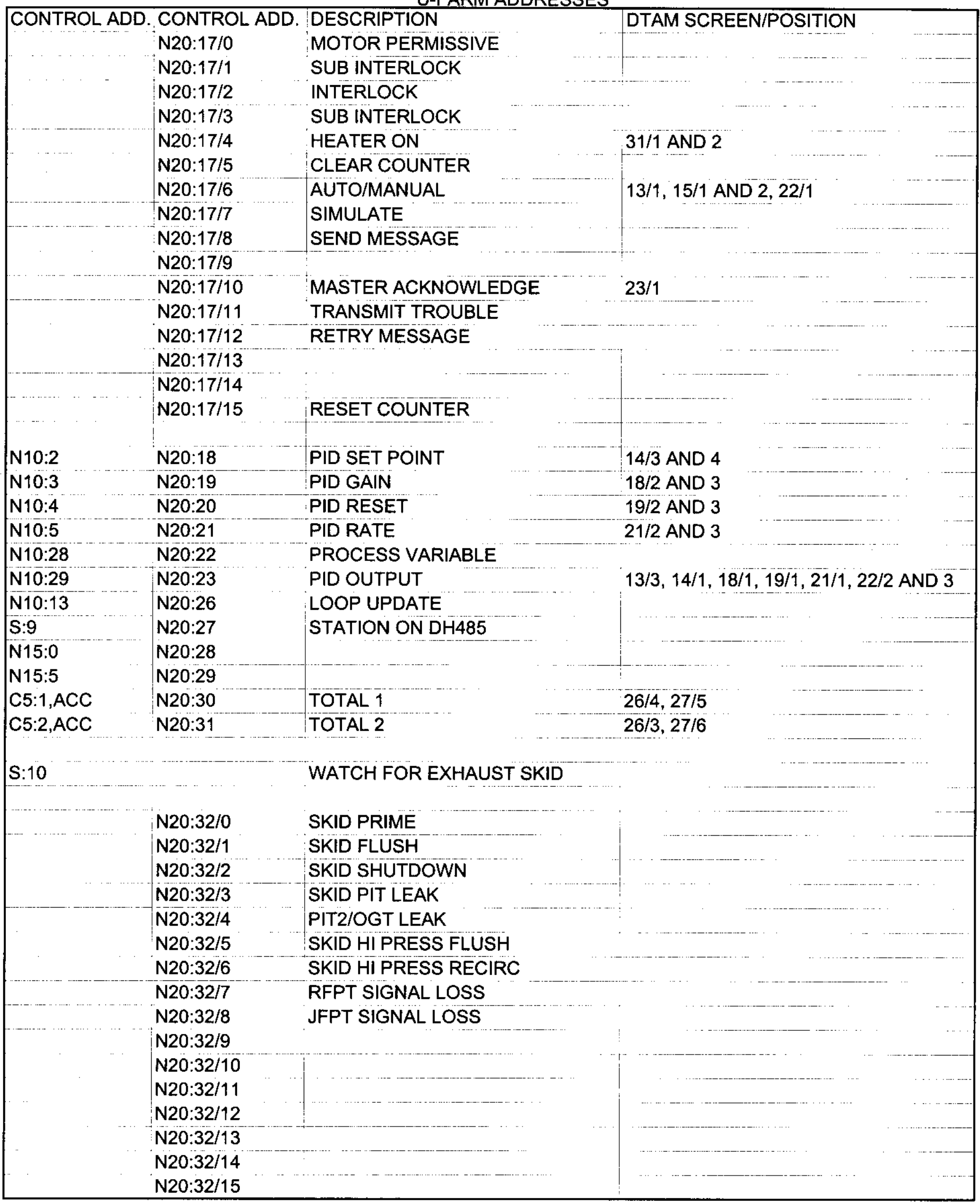




\section{RPP-5492 REVISION 0}

APPENDIX A

U-FARM ADDRESSES

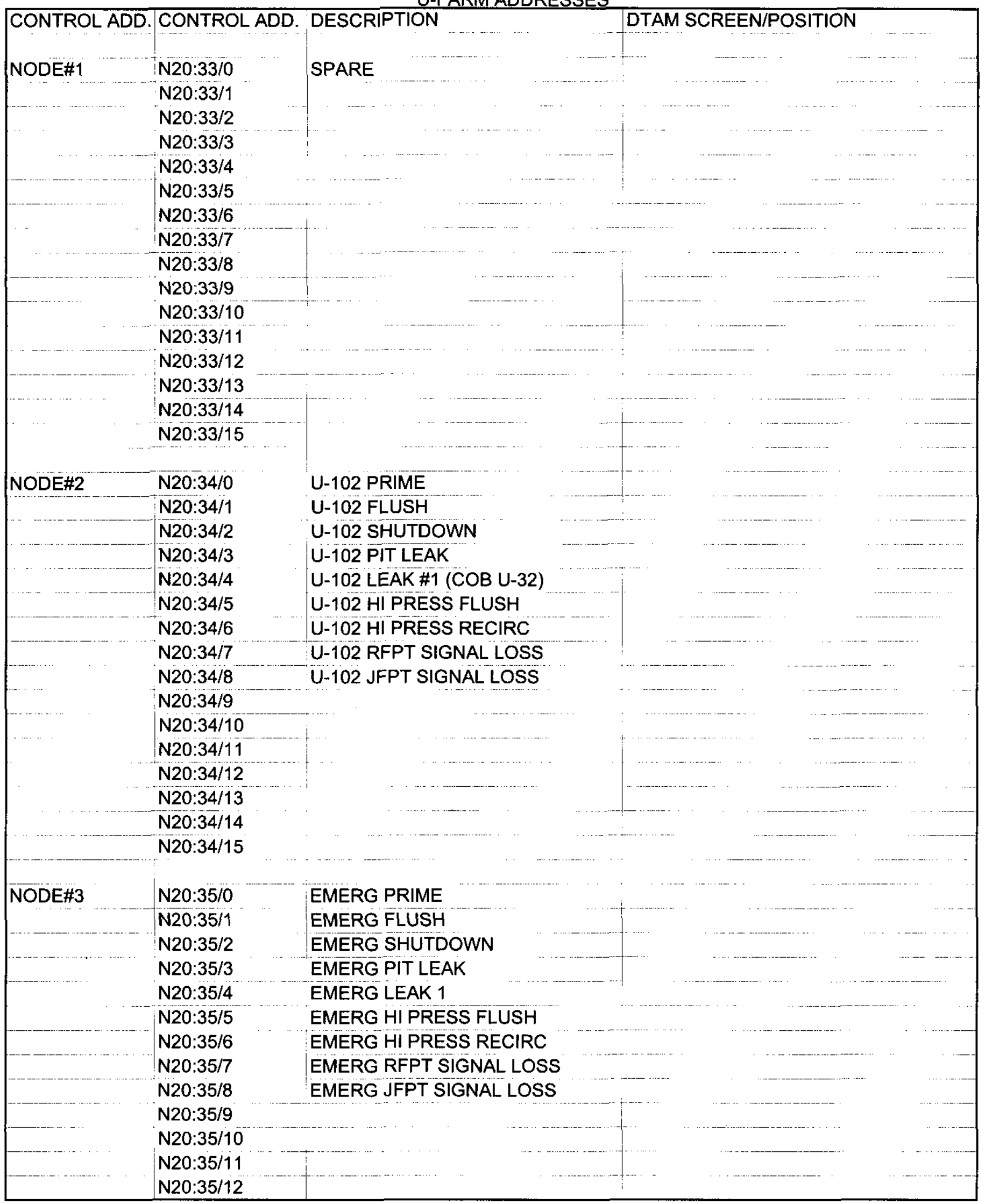


RPP-5492 REVISION 0

APPENDIX A

U-FARM ADDRESSES

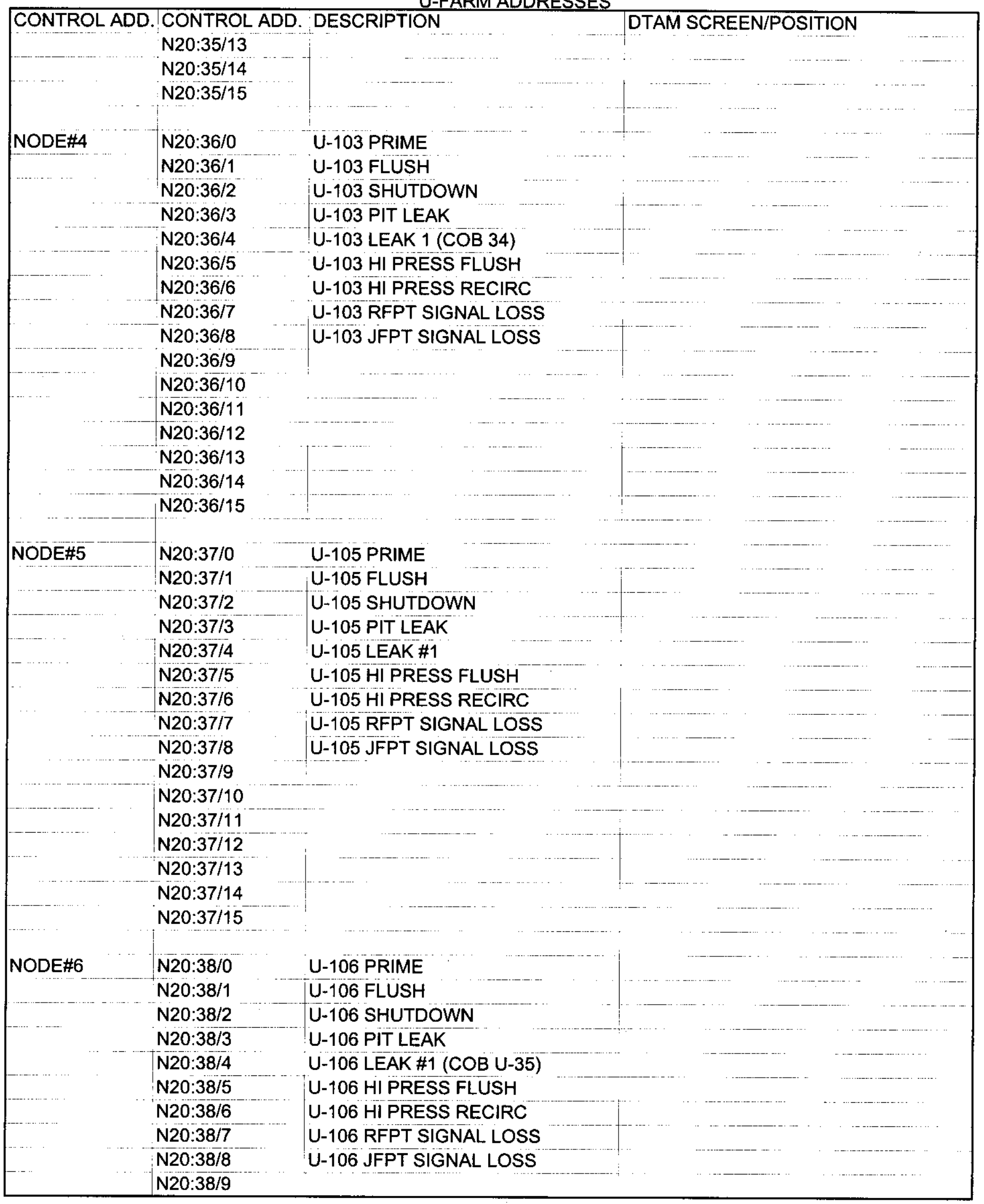




\section{RPP-5492 REVISION 0 APPENDIX A}

U-FARM ADDRESSES

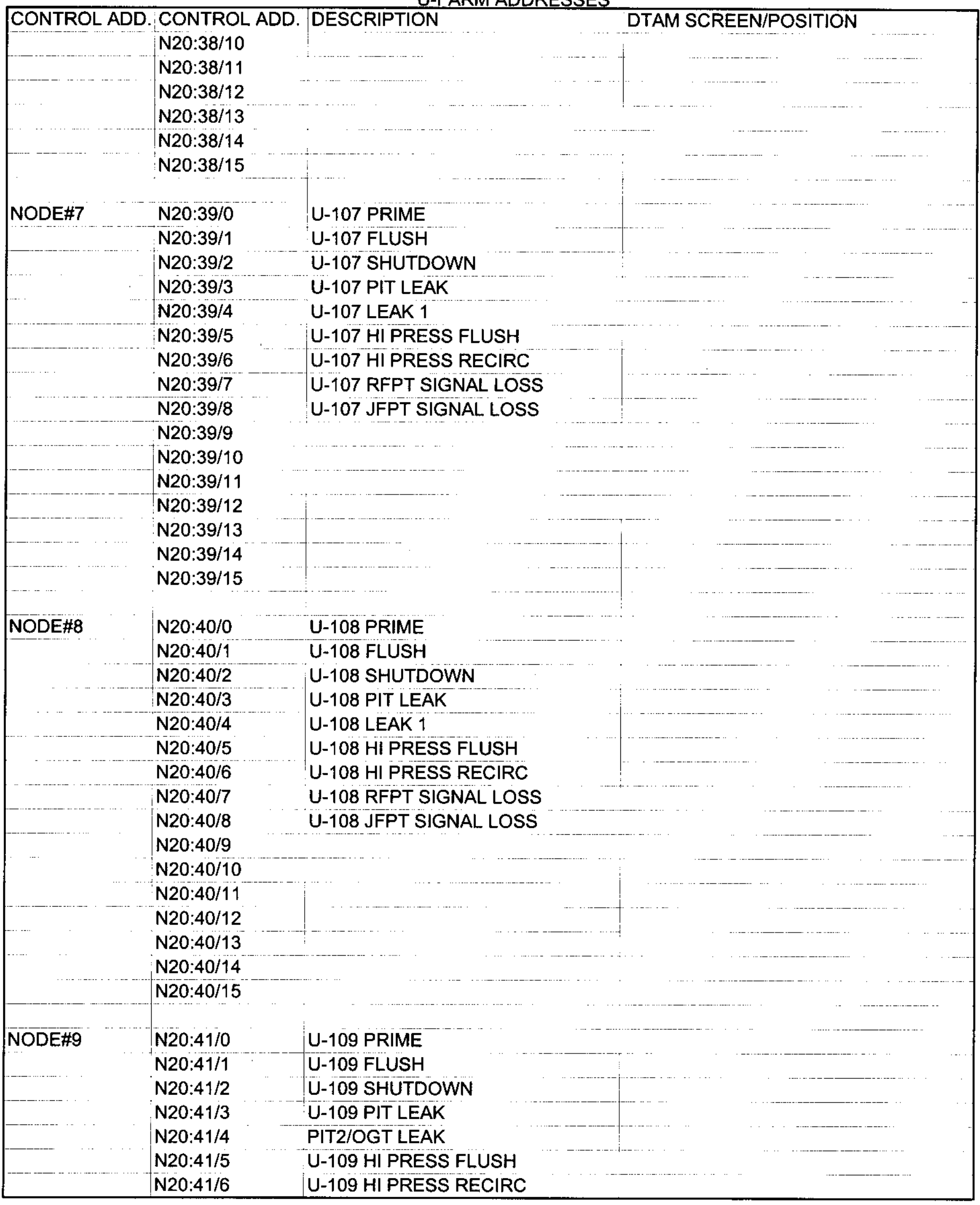


RPP-5492 REVISION 0

APPENDIX A

U-FARM ADDRESSES

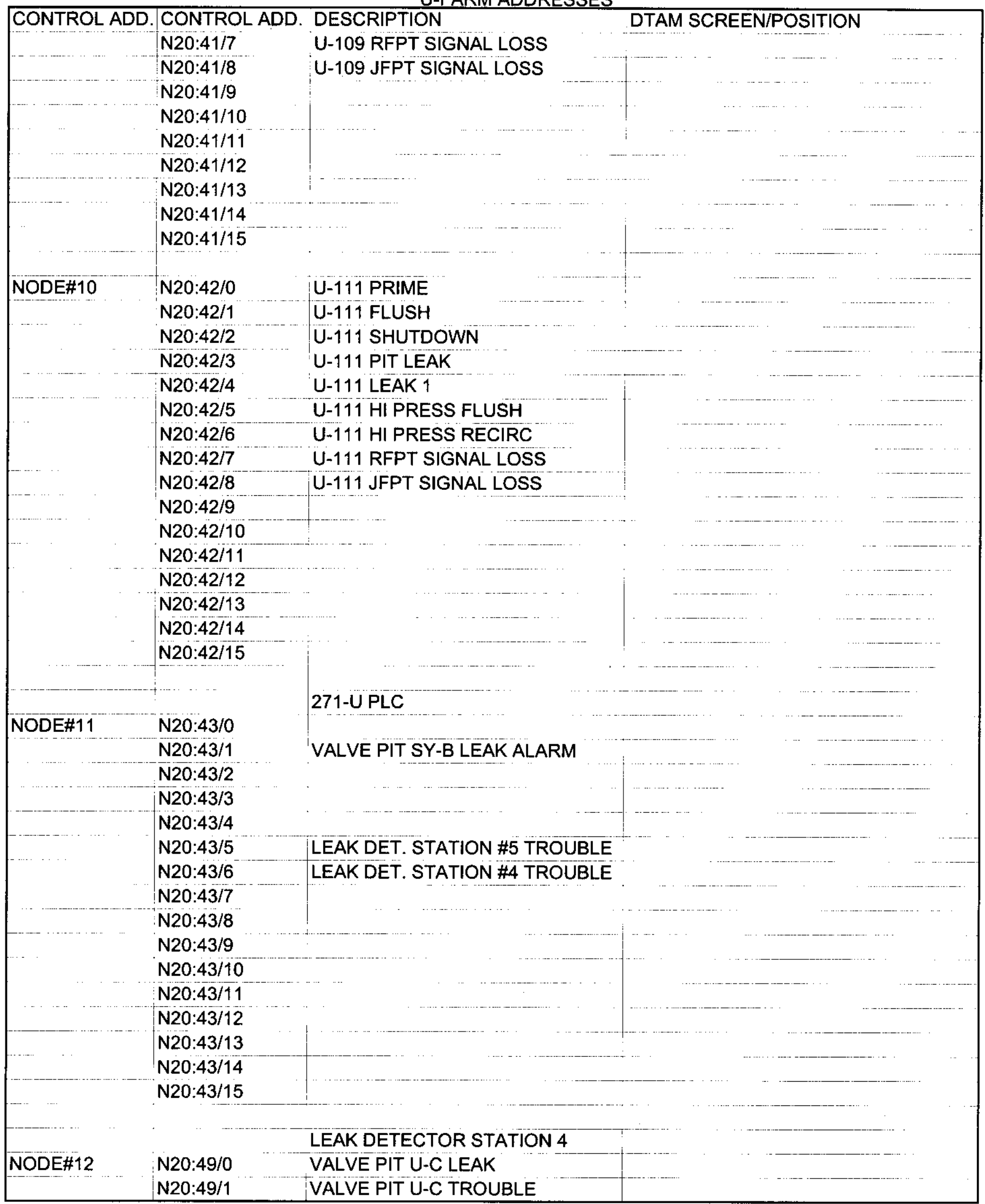




\section{RPP-5492 REVISION 0 APPENDIX A}

U-FARM ADDRESSES

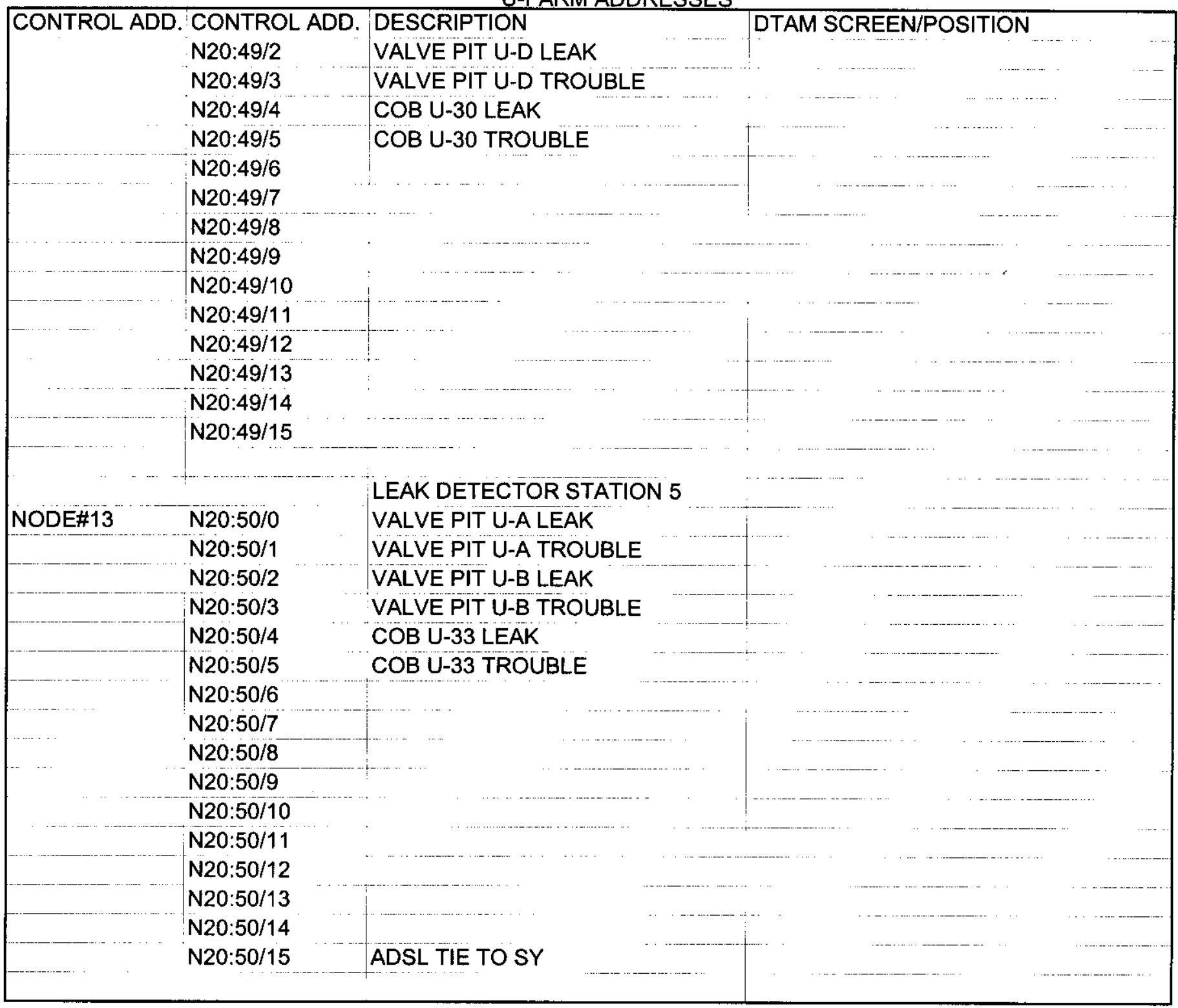


LAD 2 - - Total Rungs in File $=18$

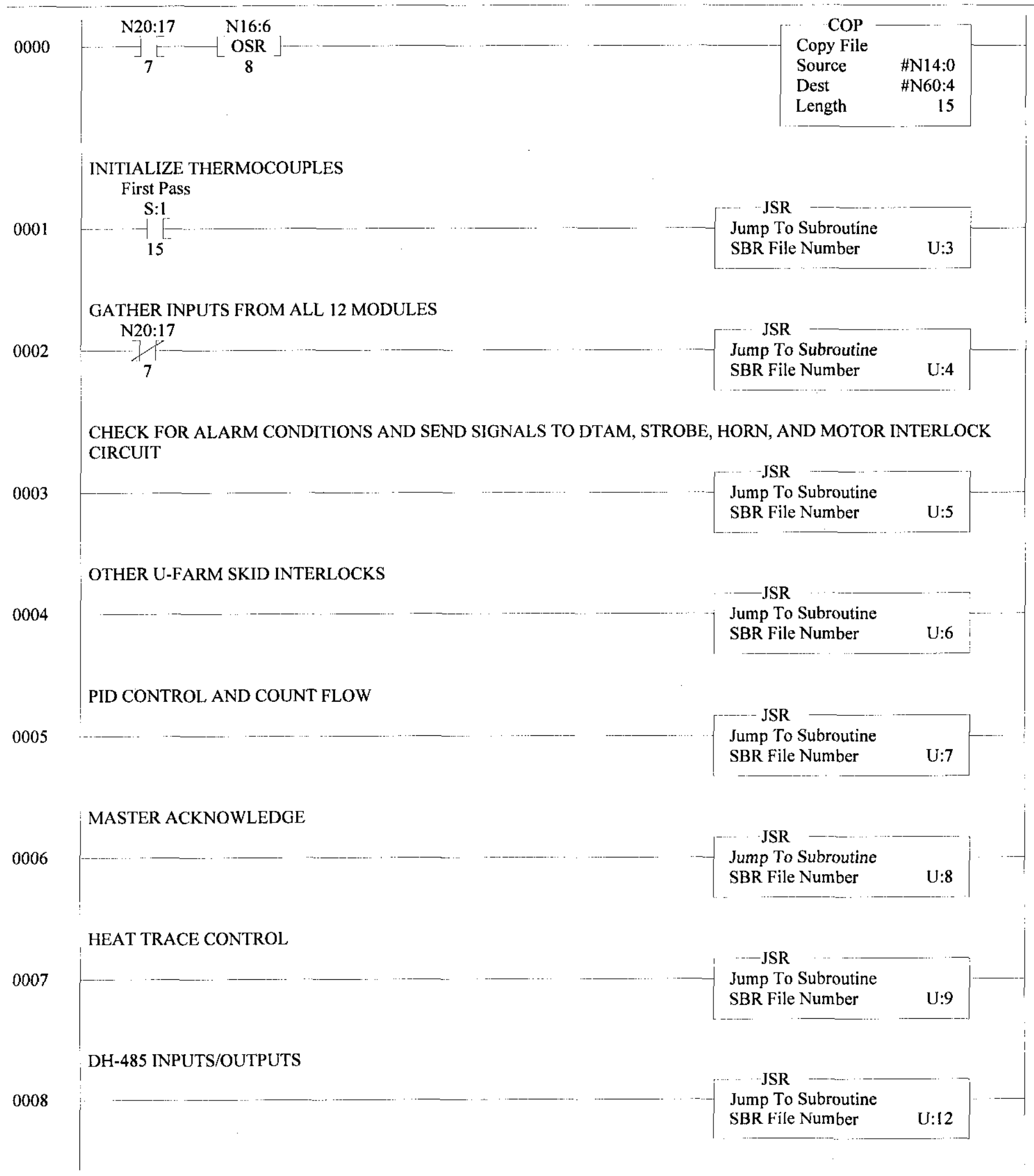




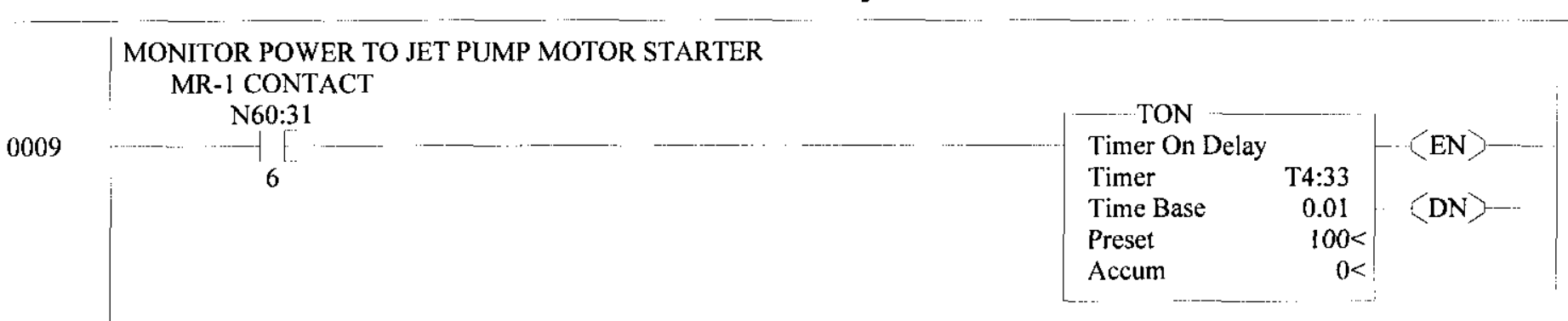

GO TO FLOW METER ROUTINE WHEN PUMP ON AND INPUT TO FLOW METER IS GREATER THAN $4 \mathrm{~mA}$ 1 SEC DELAY

0010

T4:33

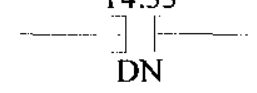

0011

NETWORK INTERLOCK

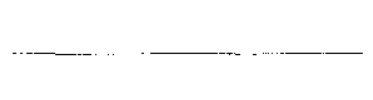

INDIVIDUAL ALARM ACKNOWLEDGE

0012

$$
\text { i. }
$$

OUTPUT TO STROBE AND LIGHTS AND HORN

0013

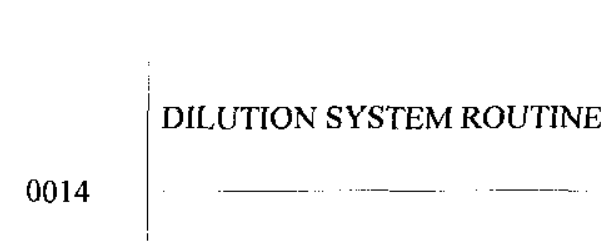

$0015 \mid \begin{gathered}\text { SEND OUTPUTS } \\ \text { N20:17 } \\ -1 / 7\end{gathered}$

RESET TOTALIZER (FLOW METER)

0016

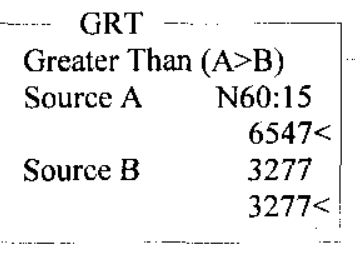

JSR

Jump To Subroutine

SBR File Number

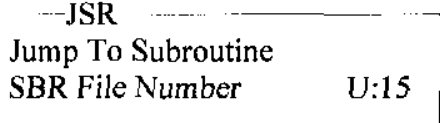

Jump To Subroutine SBR File Number

T4:33 


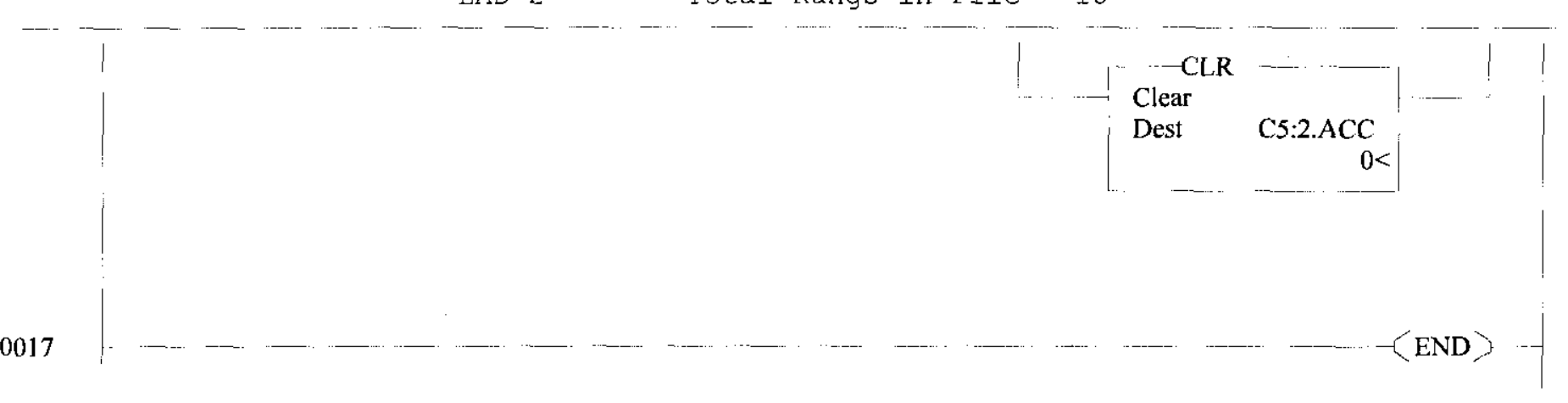

08:14:14 PAgE 3 Wednesday, March 15, 2000PAGE B_3 OF B 90 
LAD 3 - -- Total Rungs in File $=16$

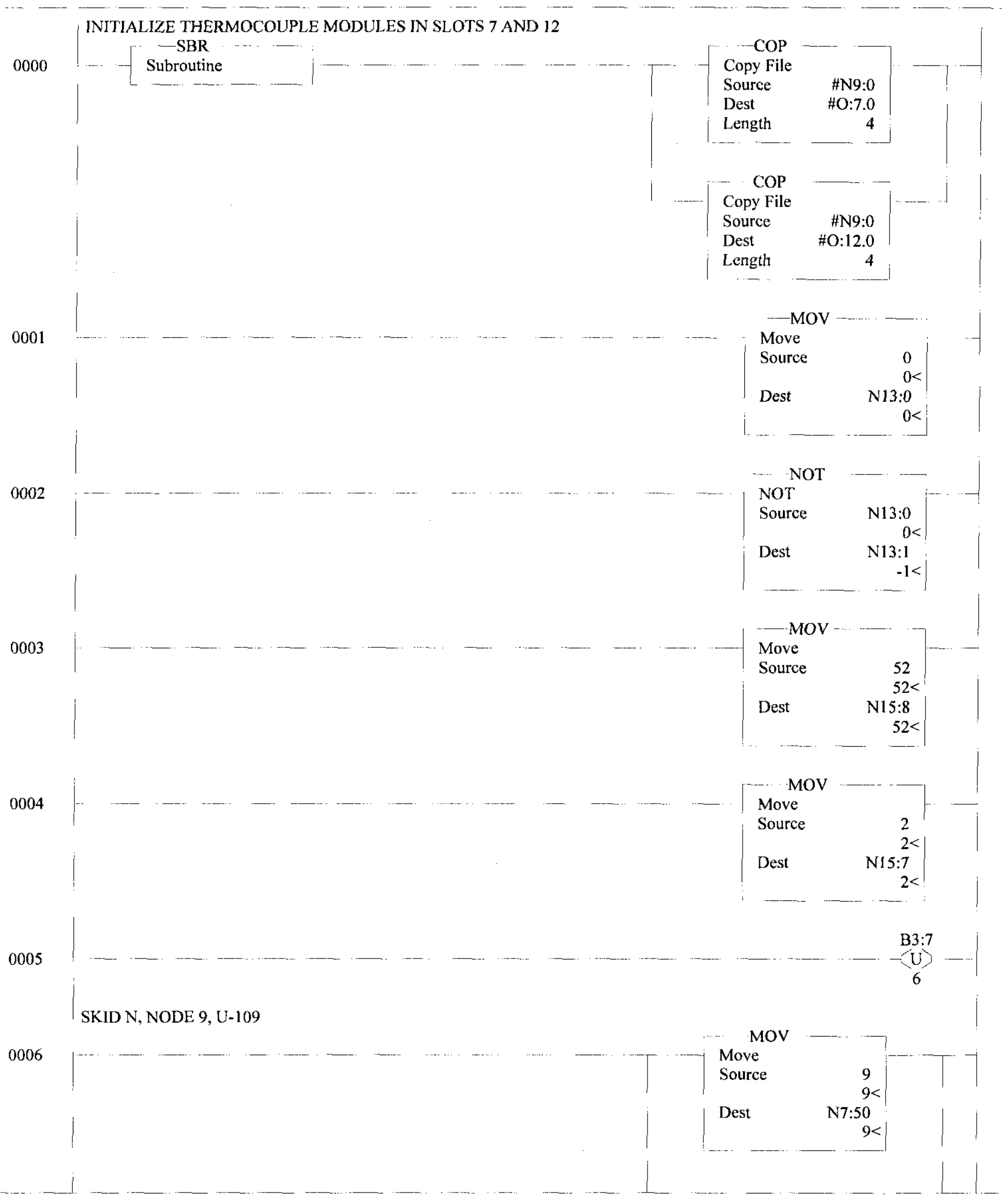




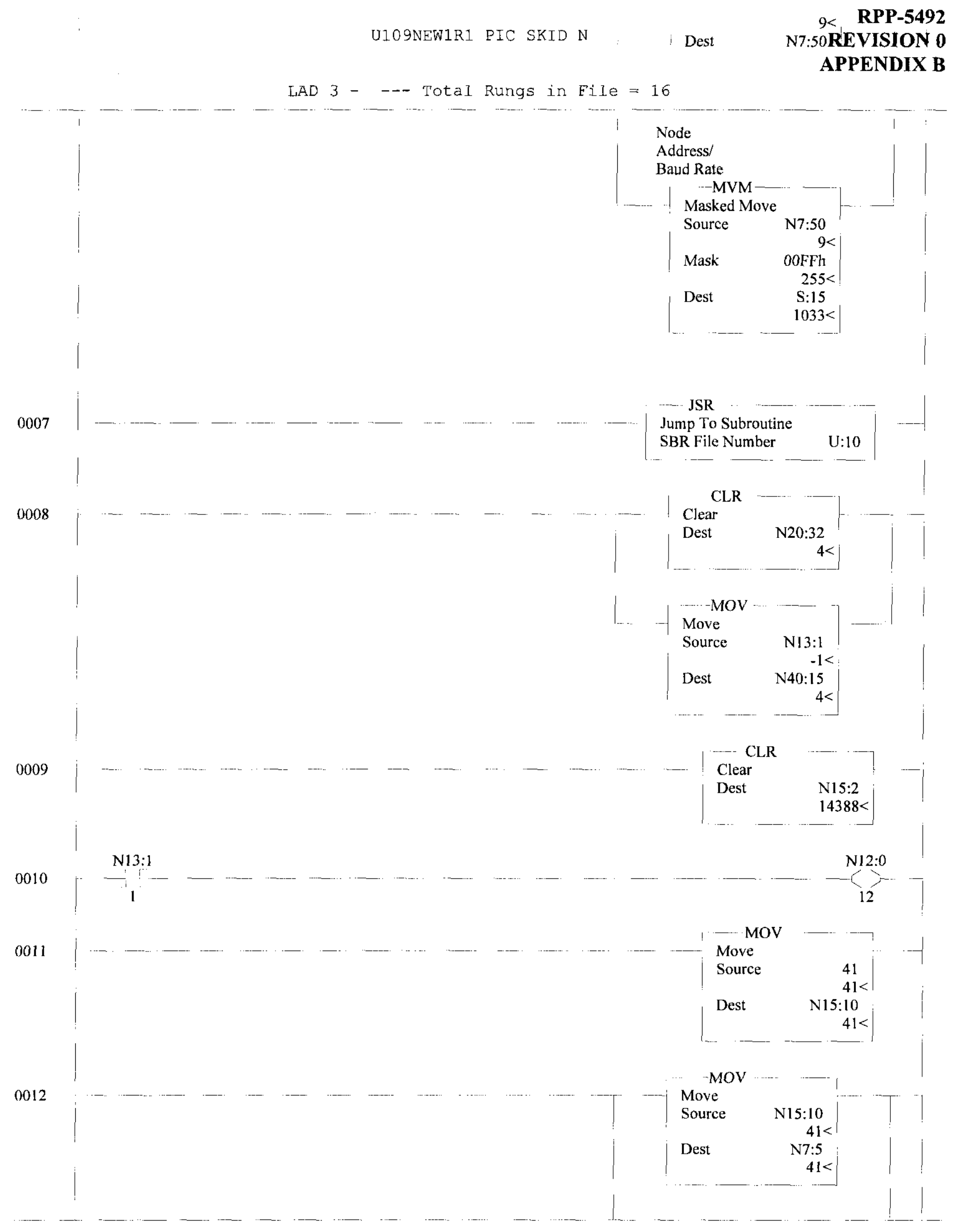

$08: 14: 14$ PAGE 2 Wednesday, $\operatorname{March}|1 \overline{5}, 2|$ MAPAGE B $\frac{5}{\sqrt{15: 10}}$ OF B 90 
LAD 3 - -- Total Rungs in File $=16$

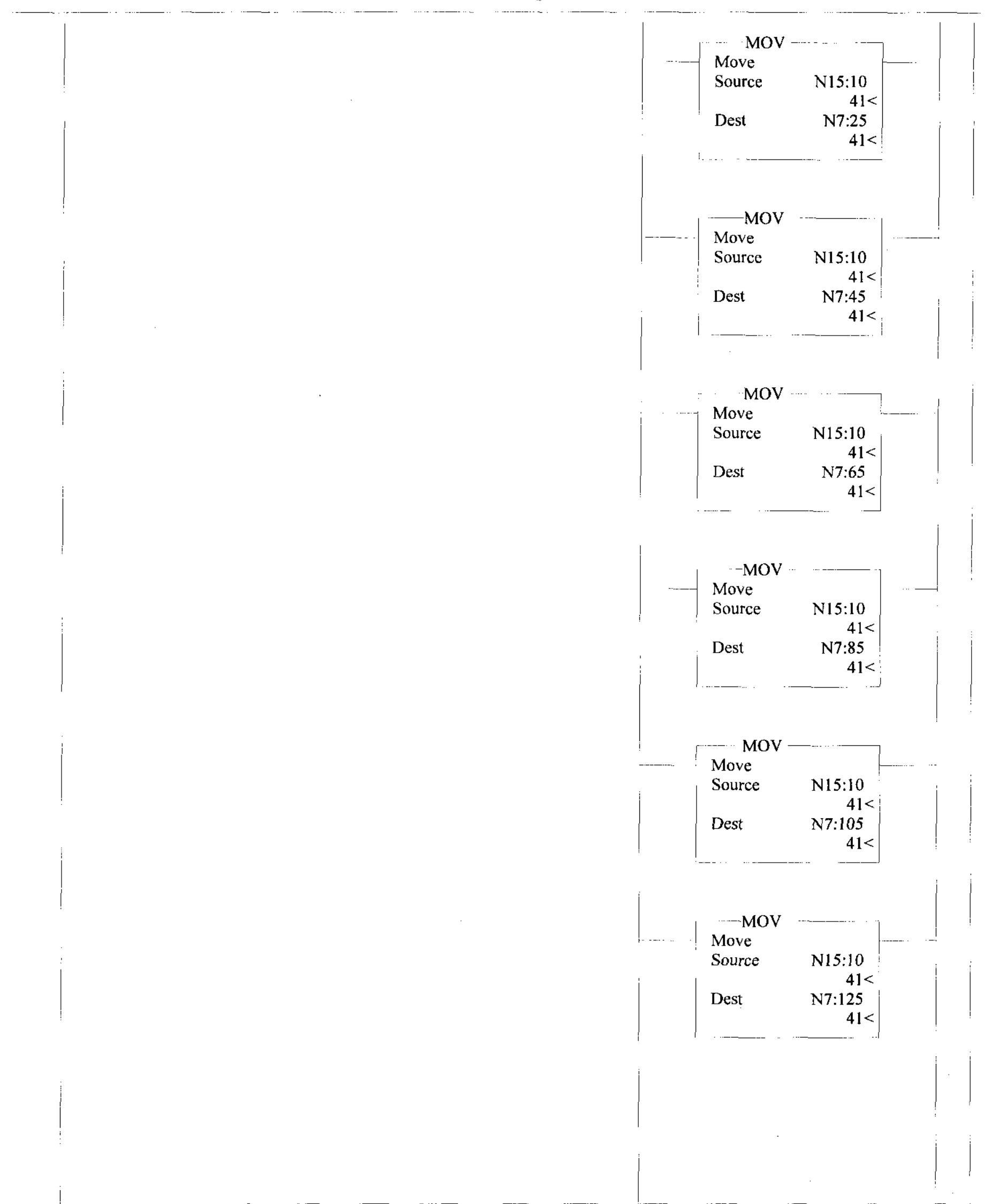

08:14:14 PAge 3 Wednesday, March $\mid 15,2000$ PAGE B 6 OF B 90 
RPP-5492
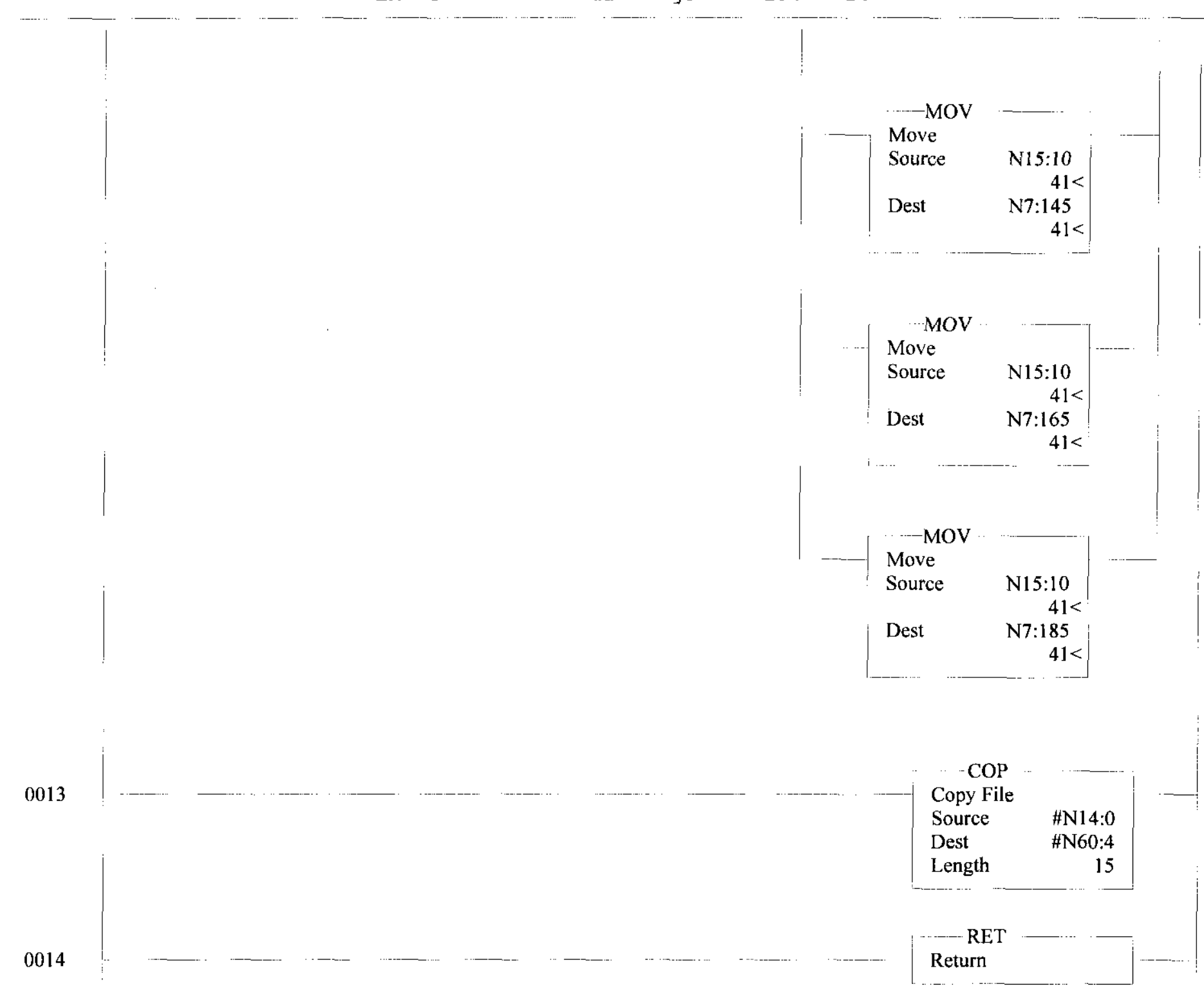

0015 


$$
\text { LAD } 4 \text { - -- Total Rungs in File }=10
$$

GET INPUTS FROM MODULES 1, 2 AND 3
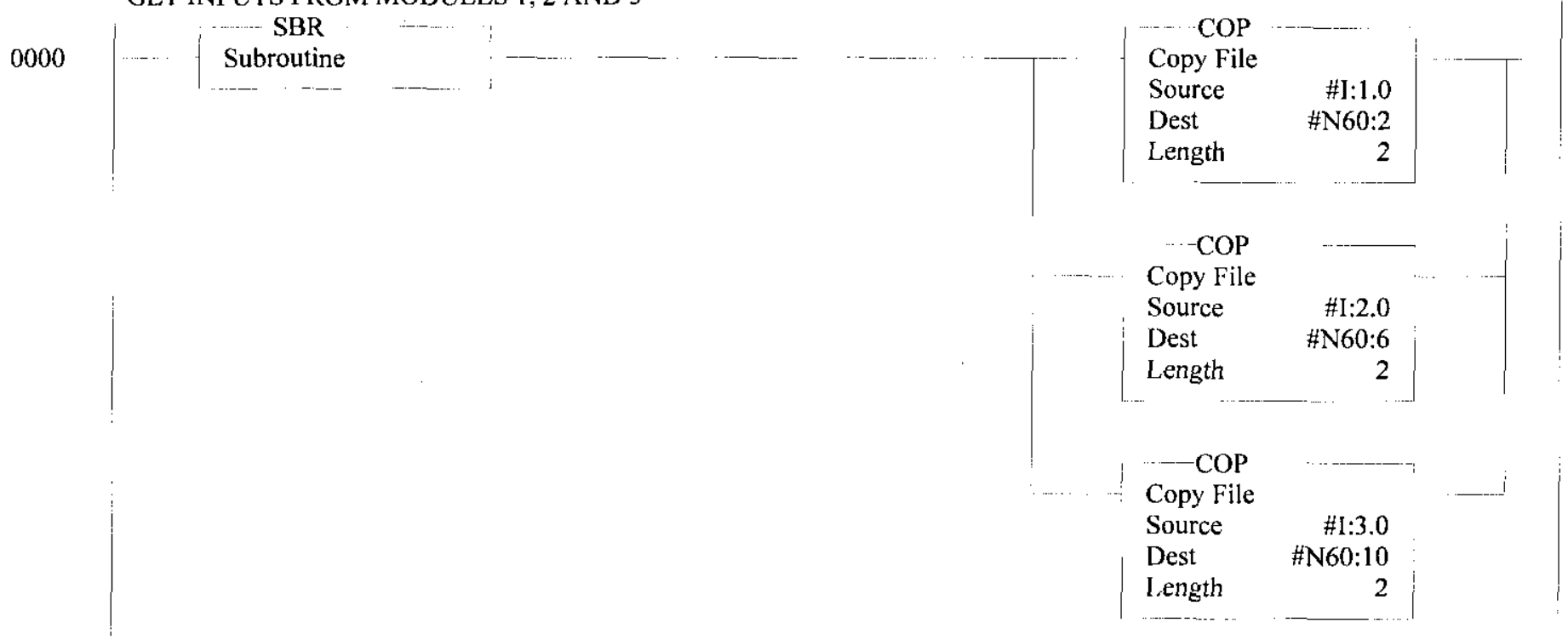

GET INPUTS FROM MODULES 4, 5 AND 6

0001

$$
\text { |... }
$$

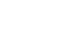


RPP-5492

LAD 4 - - Total Rungs in File $=10$

! GET INPUTS FROM MODULE 8

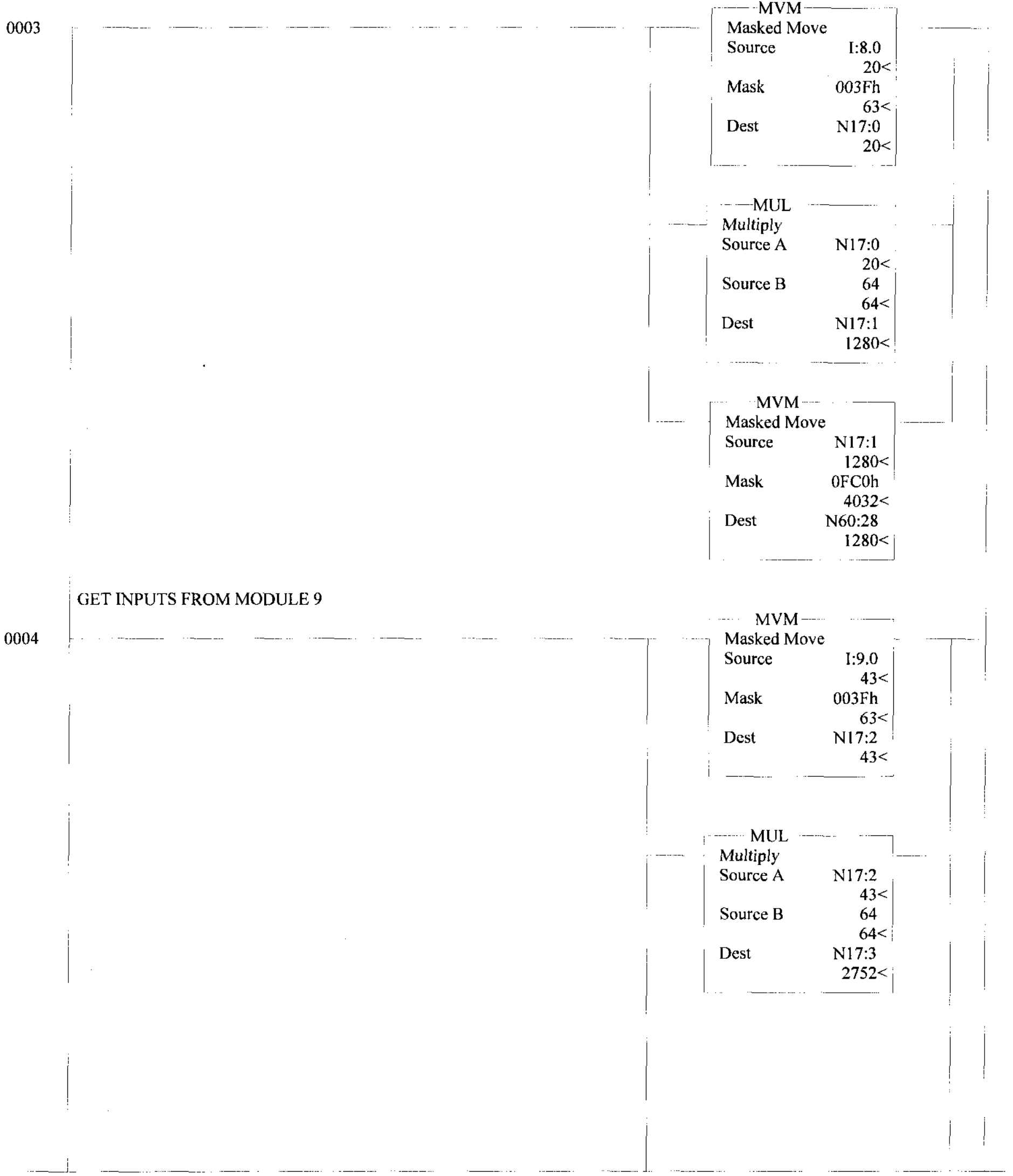




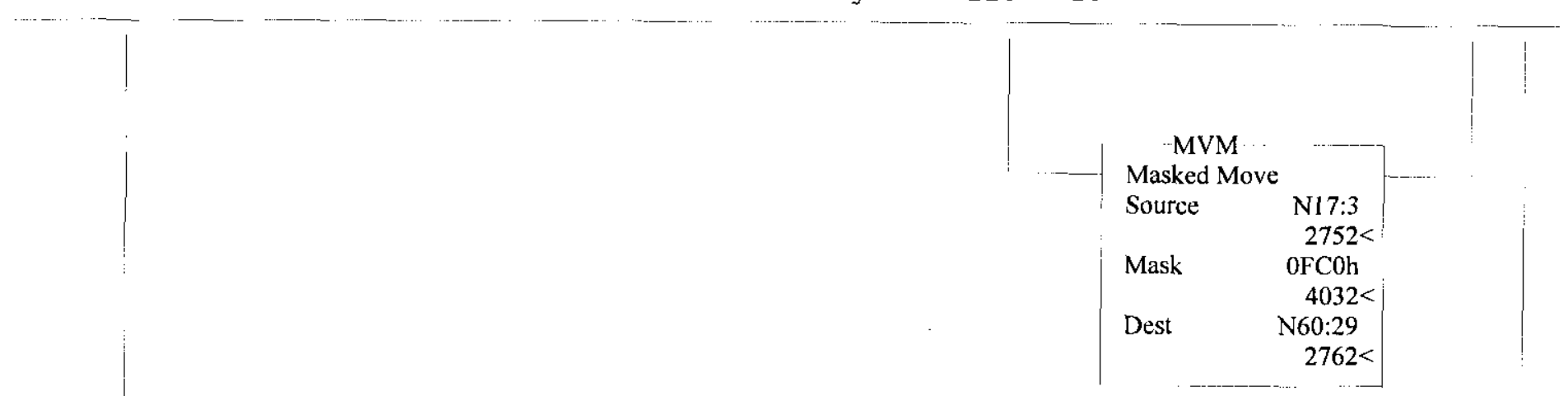

GET INPUTS FROM MODULE 10

0005
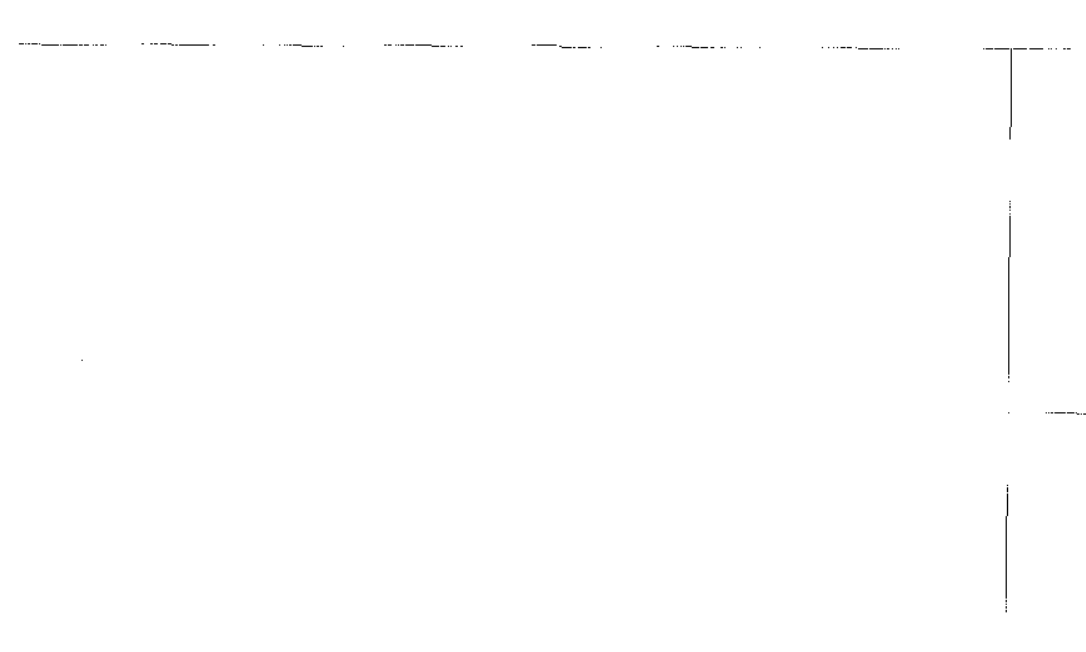

Masked Move
Source

I: 10.0

Mask

$003 \mathrm{Fh}$

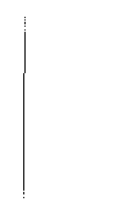

Dest $63<$

N17:4

$20<$

-...-MUL Multiply Source A

Source B

Dest

N17:5
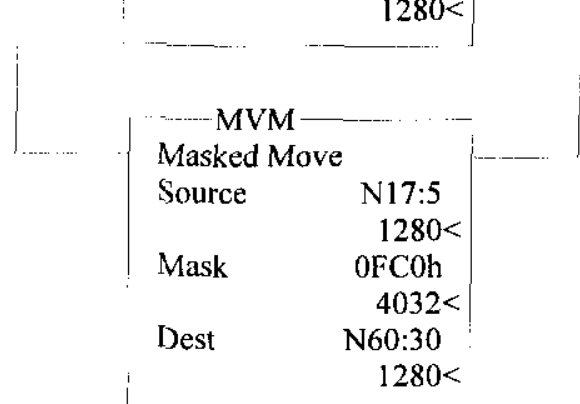

GET INPUTS FROM MODULE 11

0006

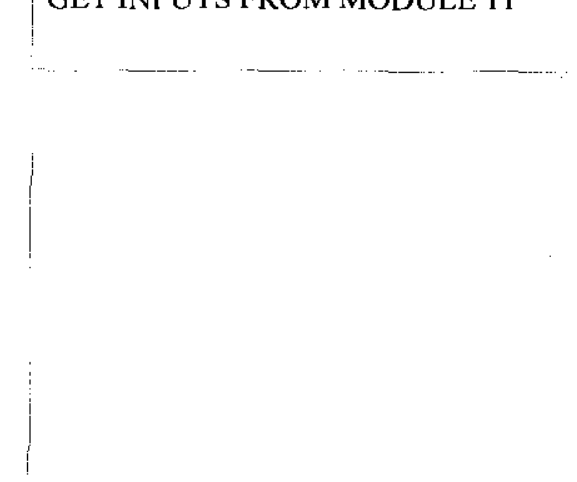

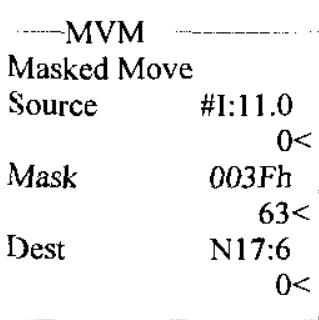

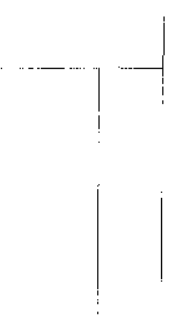

08: 14:15 PAge 3 Wednesday, March|15, 2000PAGE B 10 OF B 90 


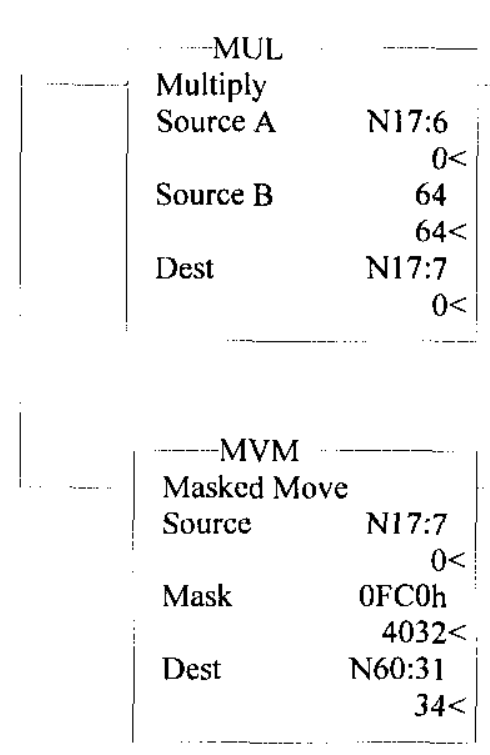

GET TEMPERATURES FROM MODULE 12

0007

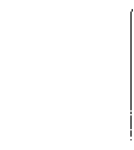

0008
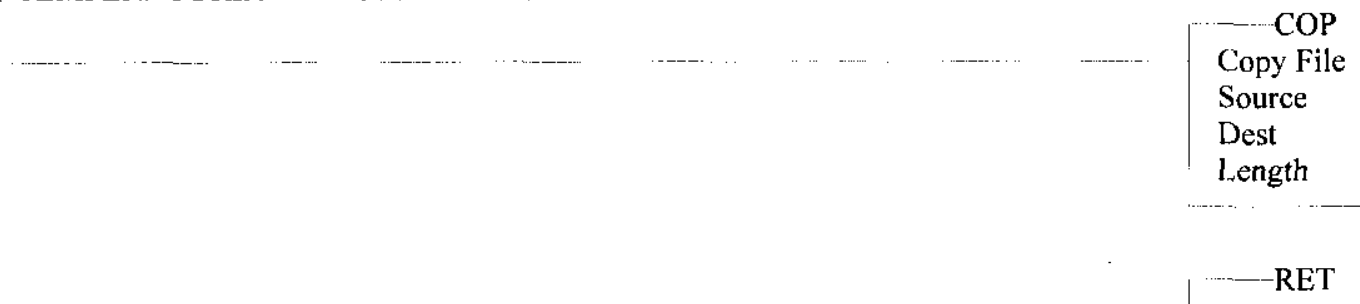

$\# \mathrm{I}: 12.0$

\#N60:32

length

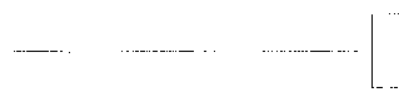

Return

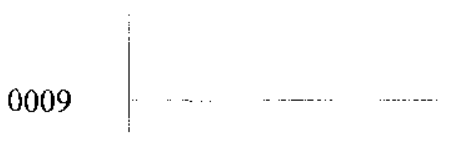

08:14:15 PAgE 4 Wednesday, March 15, 2000PAGE B $\|$ OF B 90 
LAD 5 - - Total Rungs in File $=110$

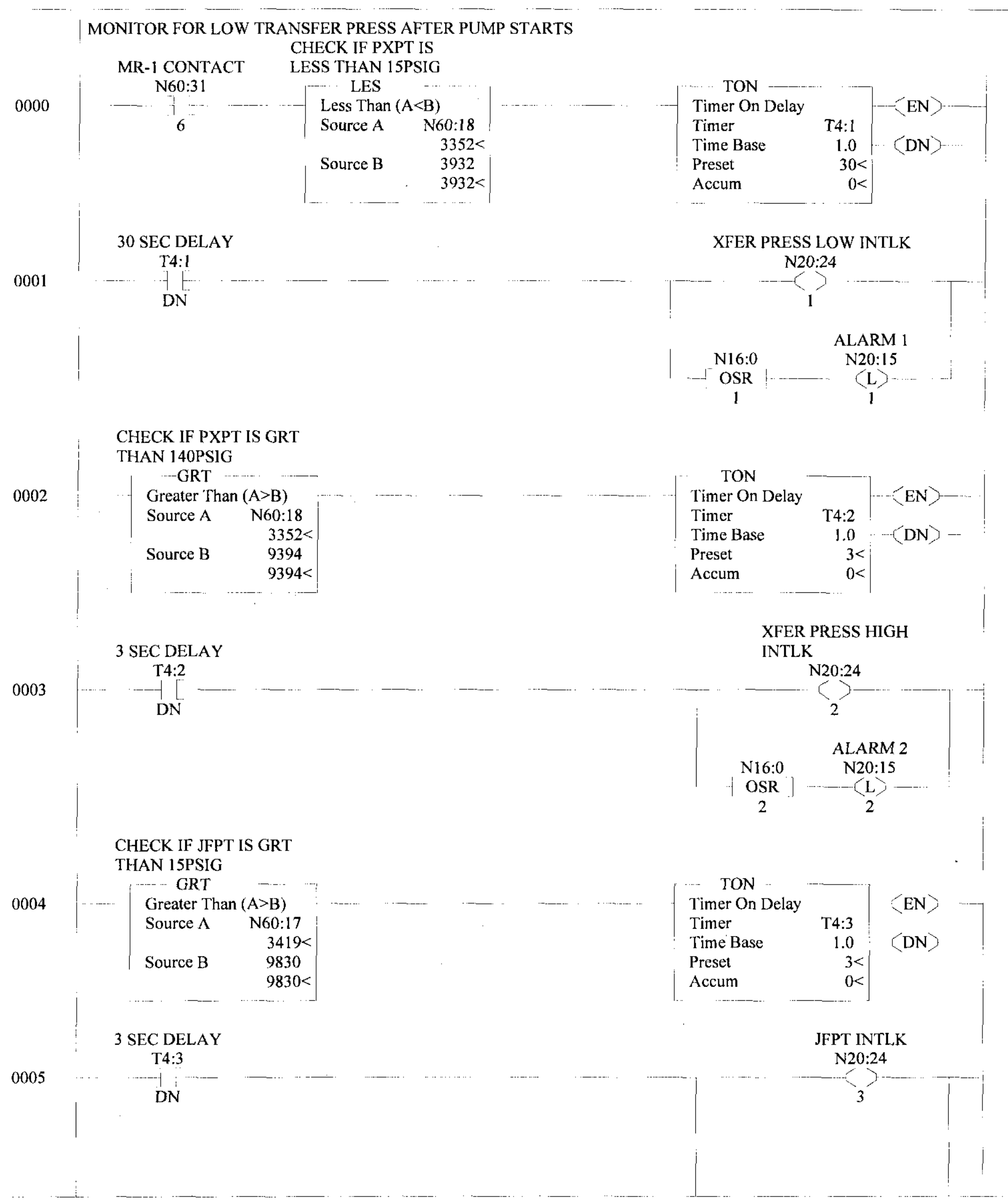

08:14:15 PAge 1 Wednesday, March 15, 2000PAGE B 12 OF B 90 

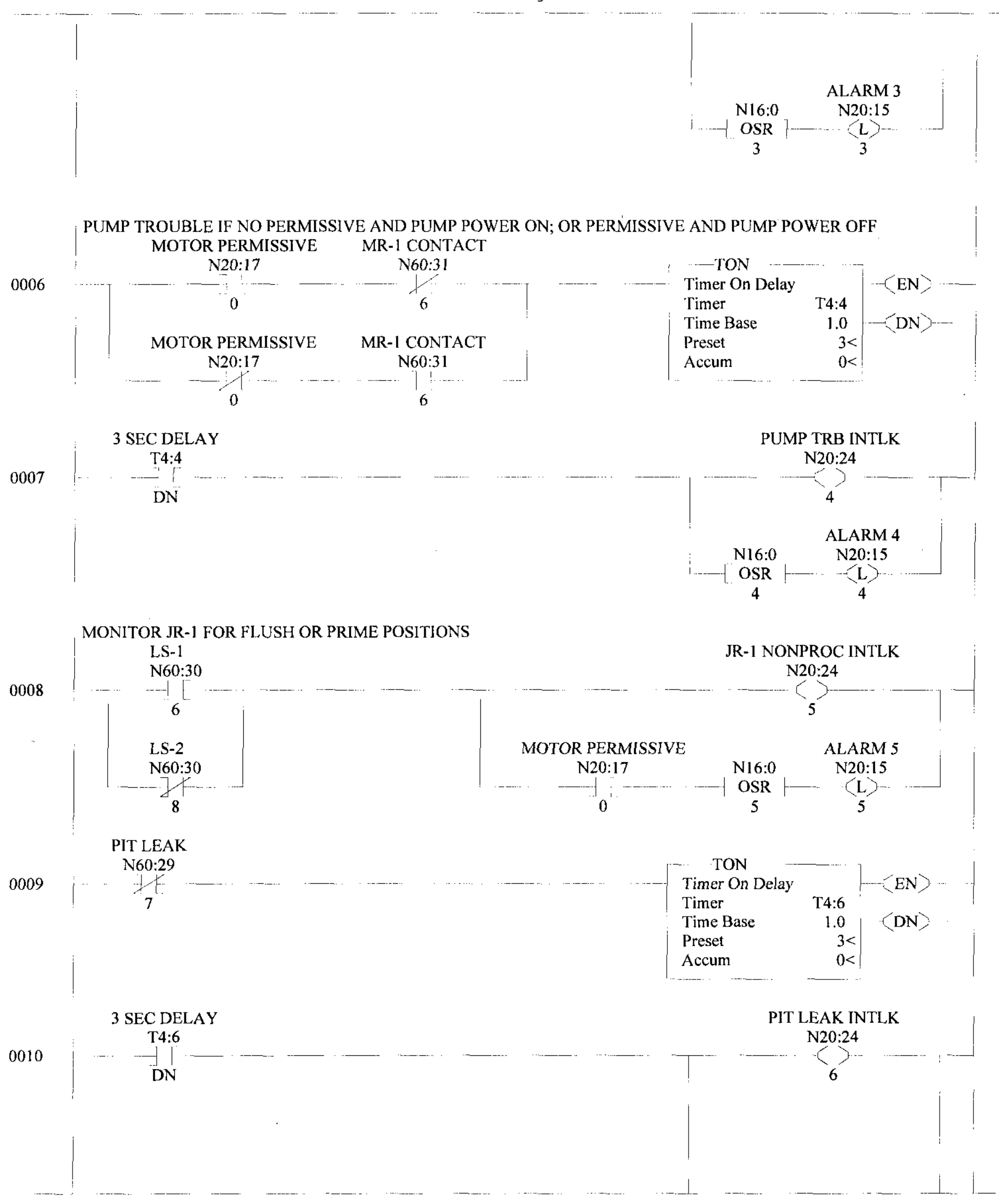


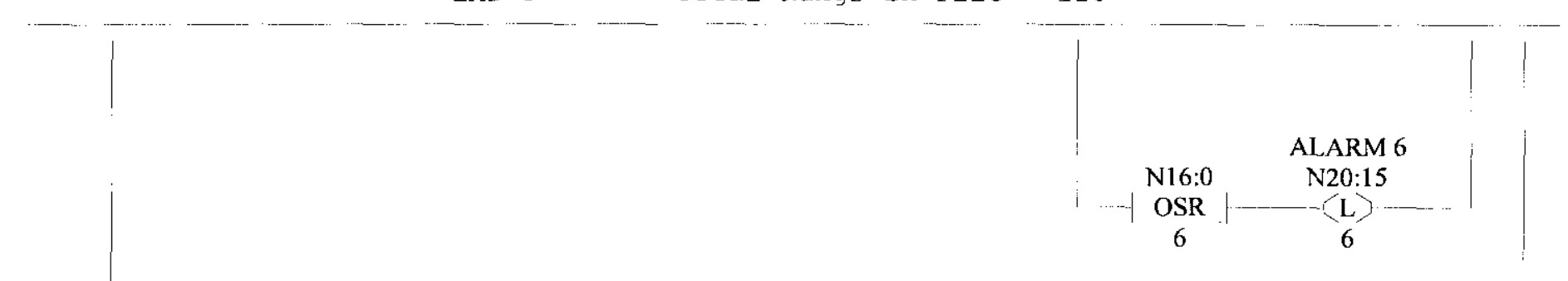

DETECTOR TROUBLE PIT LEAK

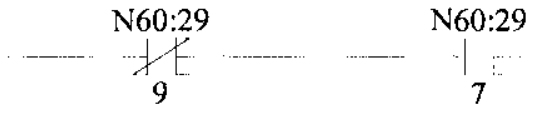

3 SEC DELAY

T4:7

0012

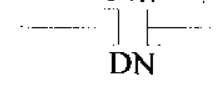
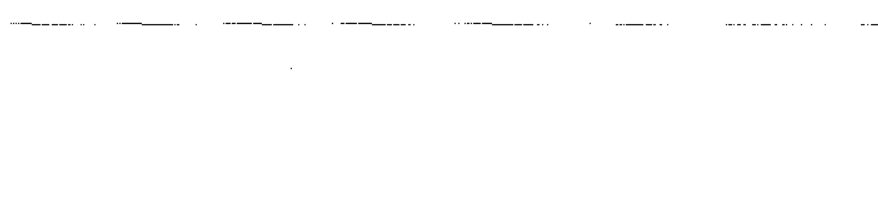

-- TON Timer On Delay

Timer

Time Base

Preset

Accum

PIT LD TRB INTLK $\mathrm{N} 20: 24$

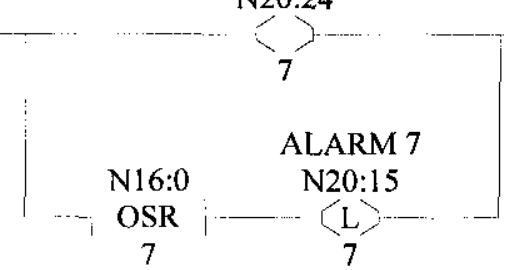

LEAK

$\mathrm{N} 60: 29$

0013

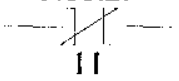

3 SEC DELAY

T4:18

0014

$+[. .$.

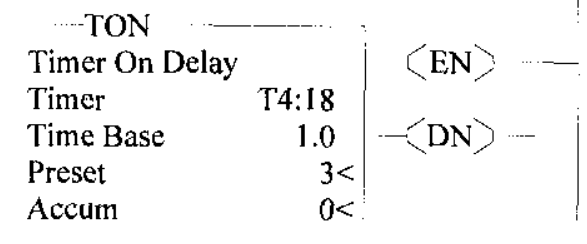

COB U-29 INTLK

N20:25

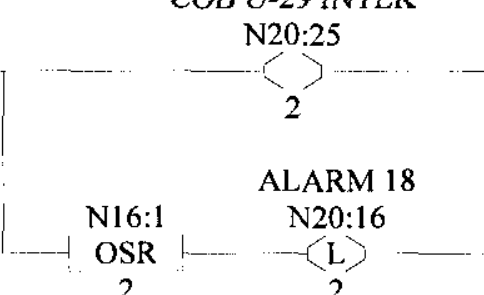

2

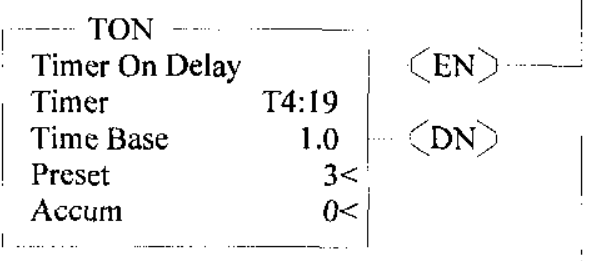


LAD 5 - - - Total Rungs in File $=110$

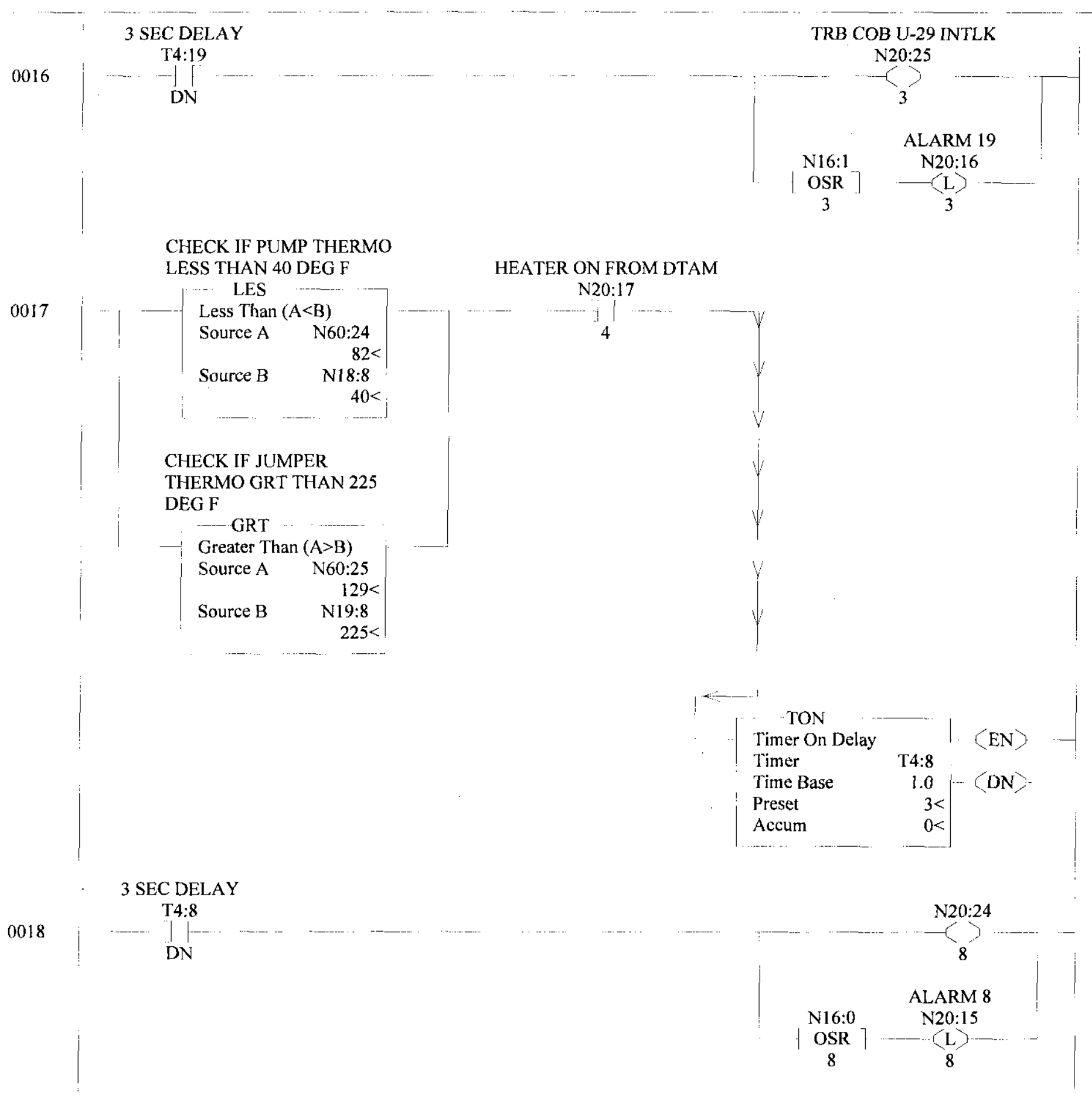




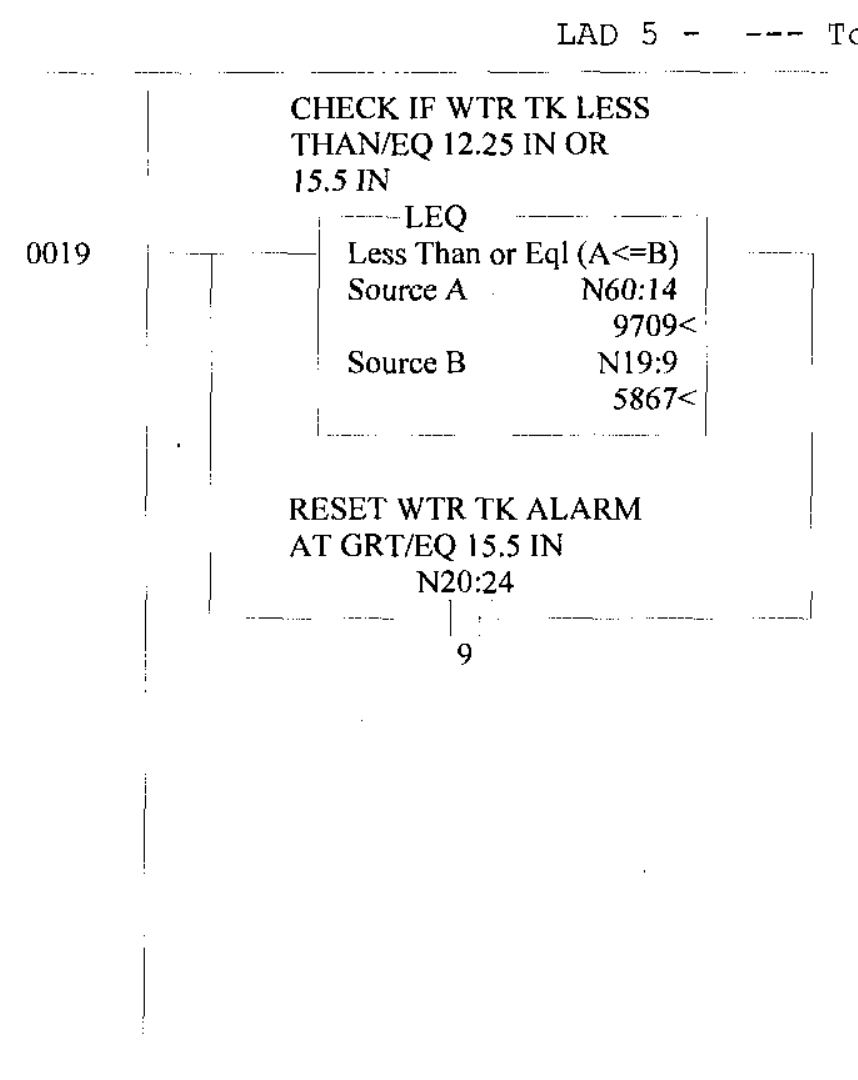

tal Rungs in File $=110$

CHECK IF WTR TK LESS
THAN/EQ 12.25 IN OR
15.5 IN

LEQ

Less Than or Eql $(\mathrm{A}<=\mathrm{B})$

Source A N60:14

$9709<$
Source B $\quad \mathrm{N} 18: 9$

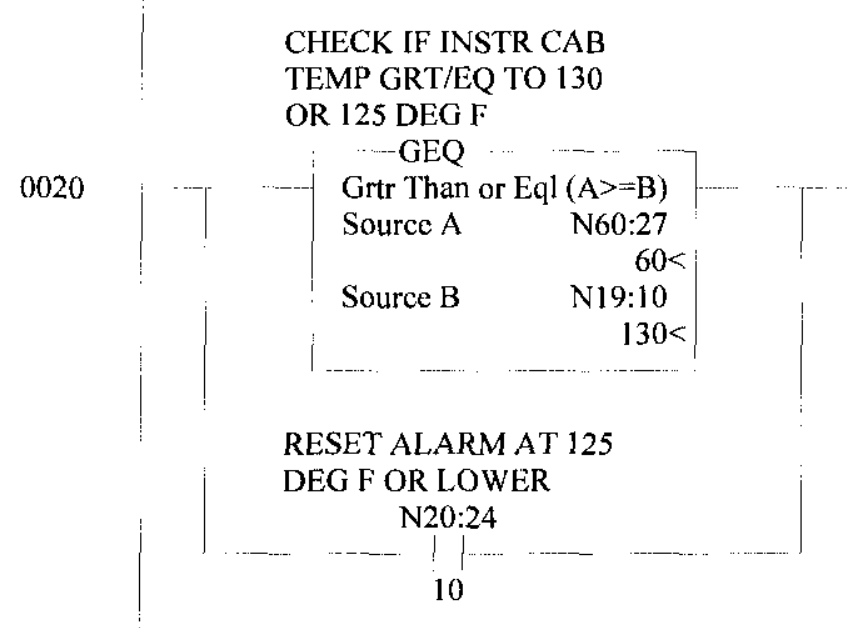

CHECK IF INSTR CAB

TEMP GRT/EQ TO 130

OR 125 DEG $F$
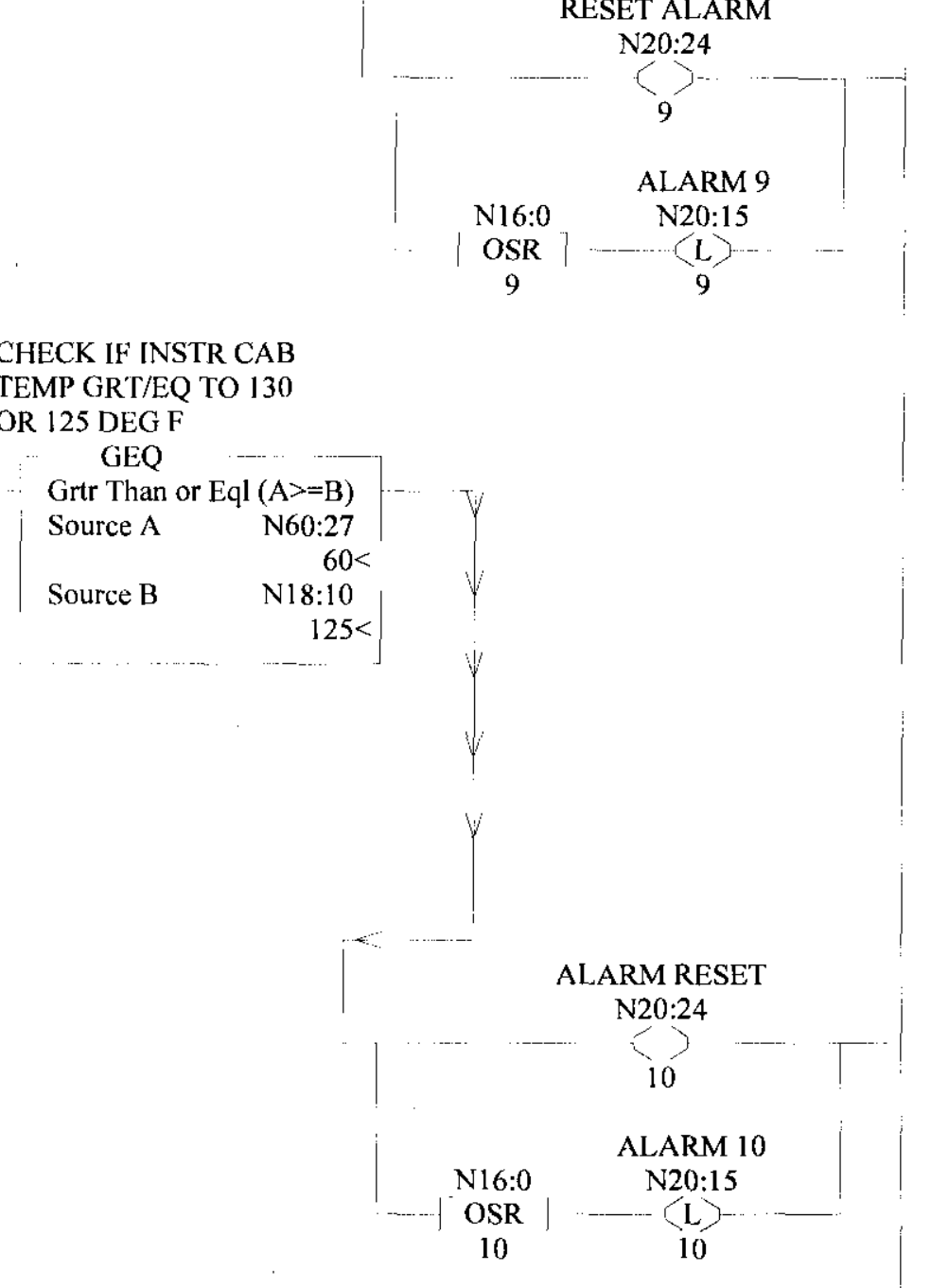

RESET ALARM

$\mathrm{N} 20: 24$

CHECK IFTNSTR CAB

Grtr Than or Eql $(\mathrm{A}>=\mathrm{B})$

N60.27

Source B N19:10

RESET ALARM AT 125

DEG F OR LOWER

()

10 
LAD 5 - -. Total Rungs in File $=110$

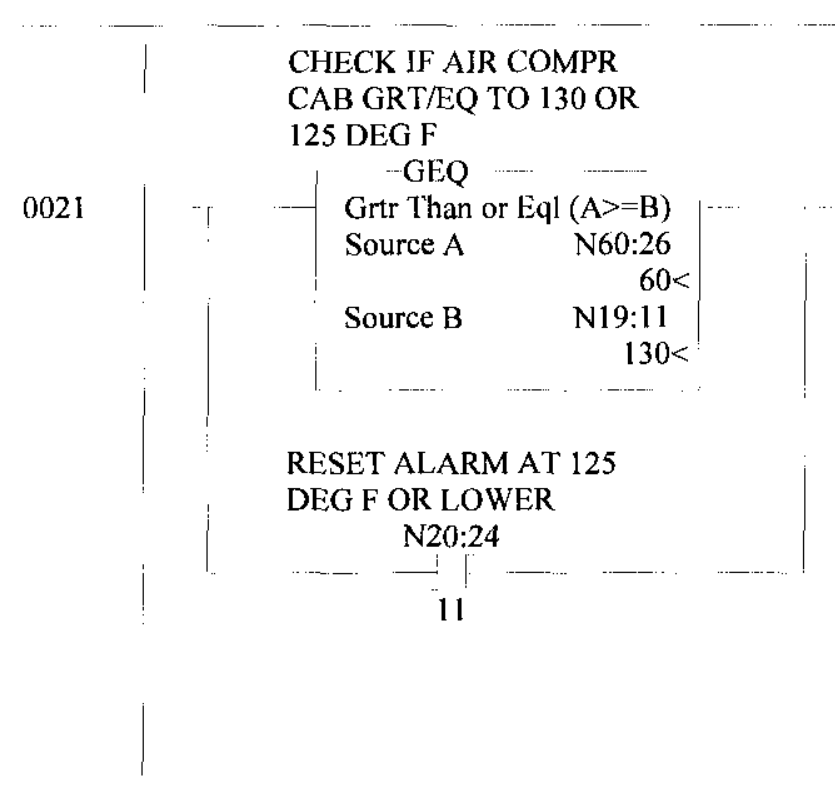

\section{CHECK IF AIR COMPR \\ CAB GRT/EQ TO 130 OR 125 DEG F}

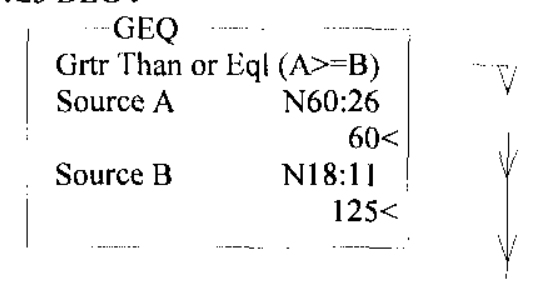

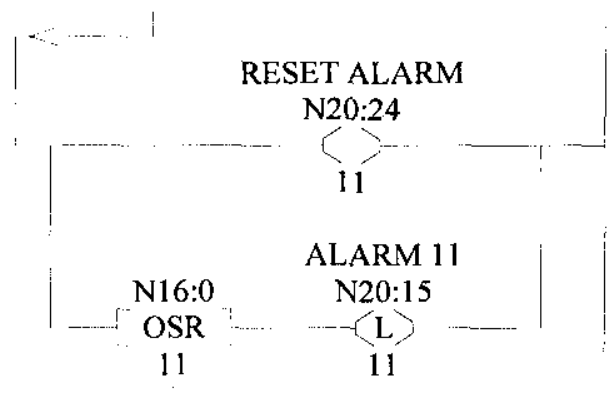

MR-1 CONTAC'T

$\mathrm{N} 60: 31$

0022
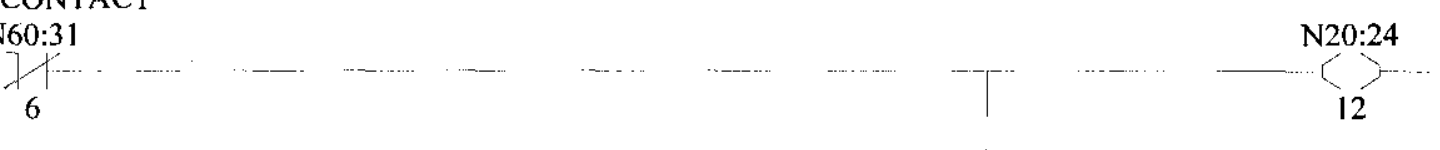

12

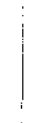
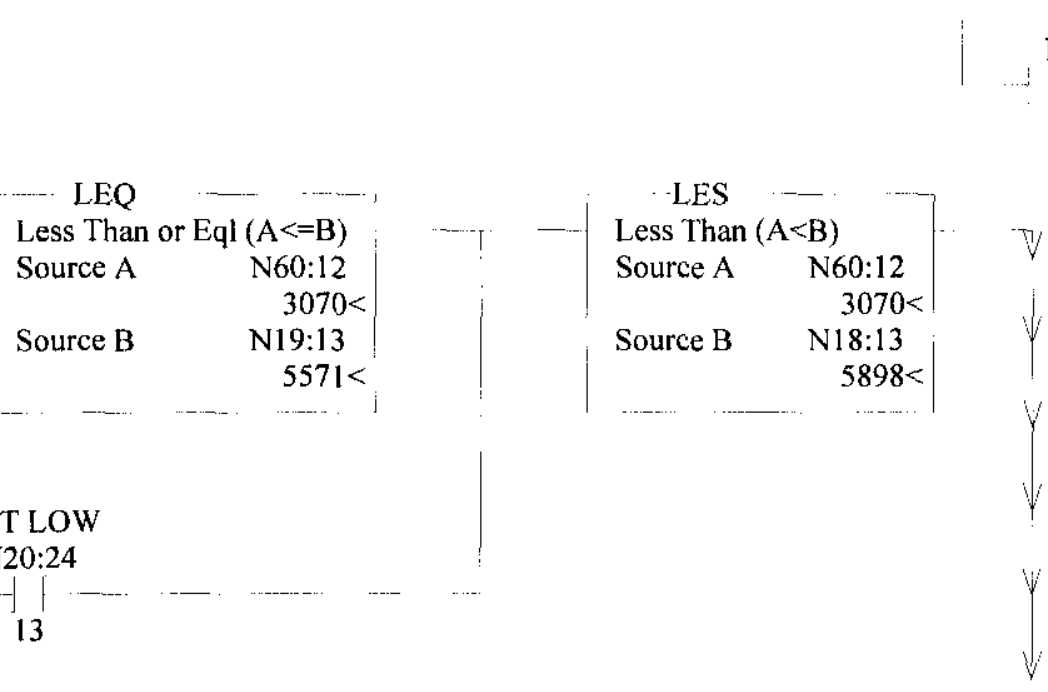

SALTWELL LOW LEVEL $\mathrm{N} 20: 24$

ALARM 12 N20:15

(L)

12

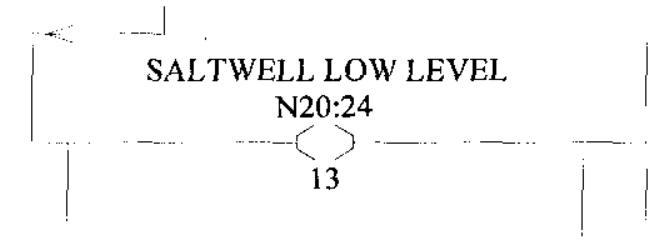




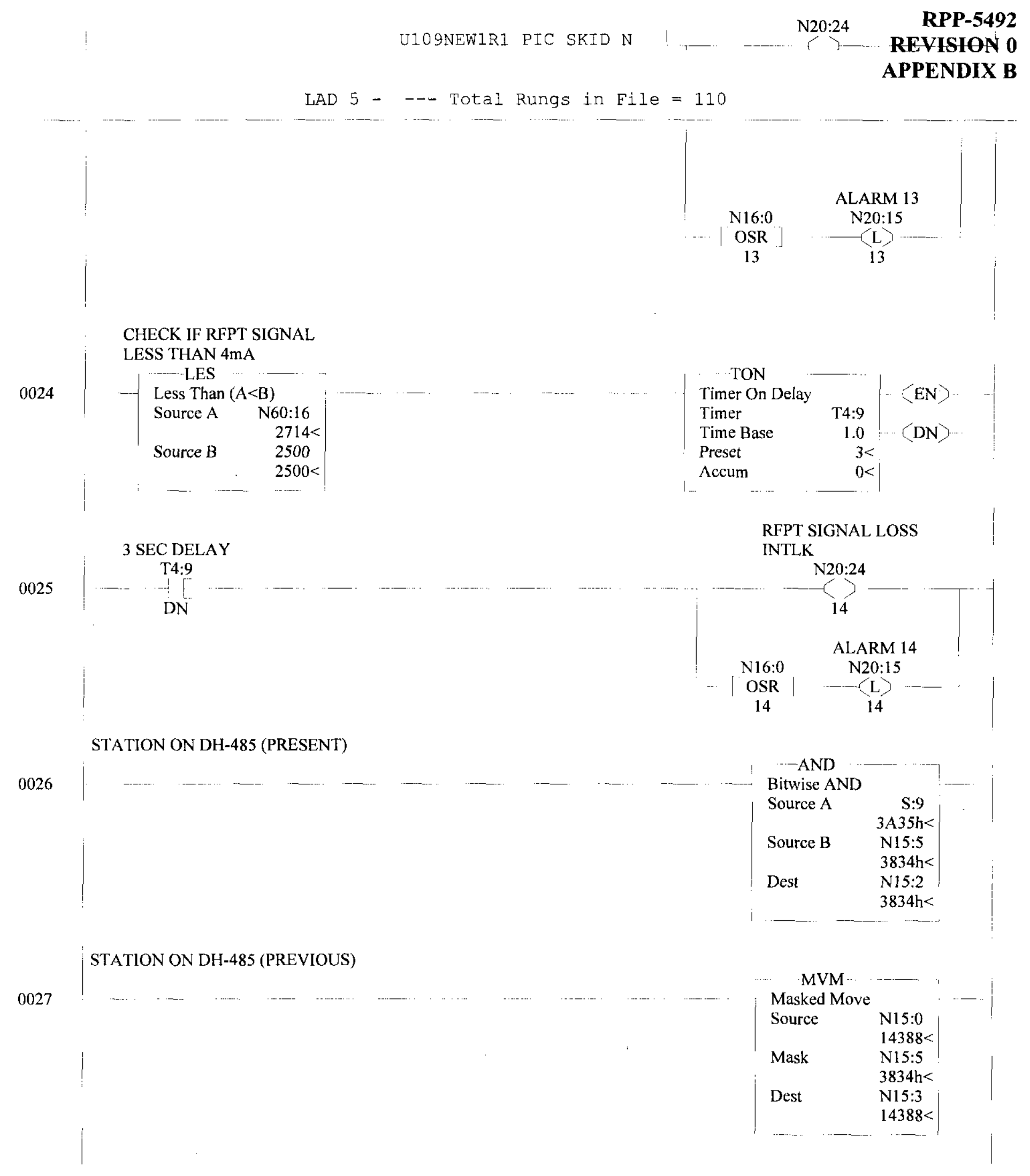

08:14:18 PAgE 7 Wednesday, March 15, 2000PAGE B 18 OF B 90 
LAD 5 - -- Total Rungs in File $=110$

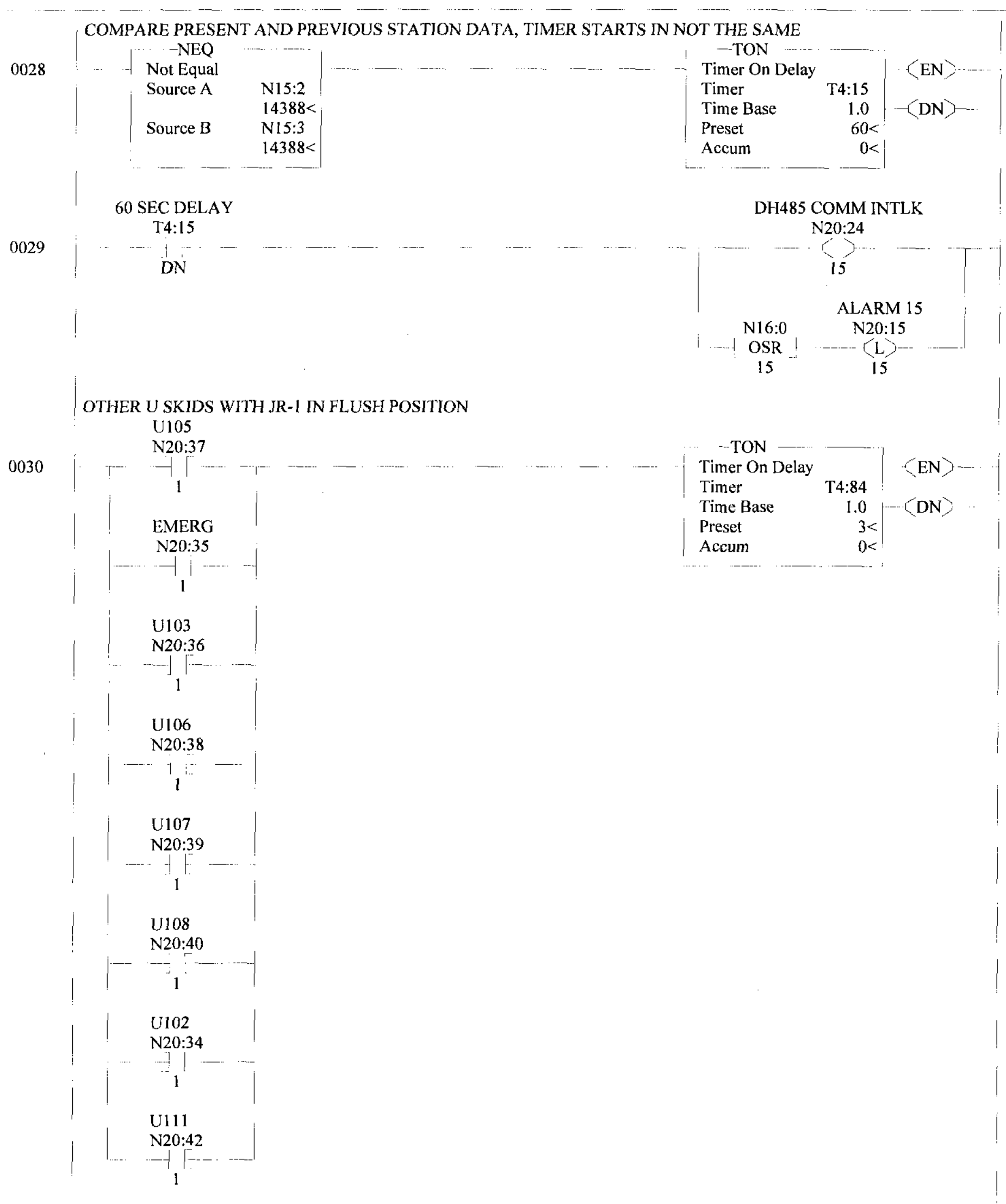


LAD 5 - --- Total Rungs in File $=110$
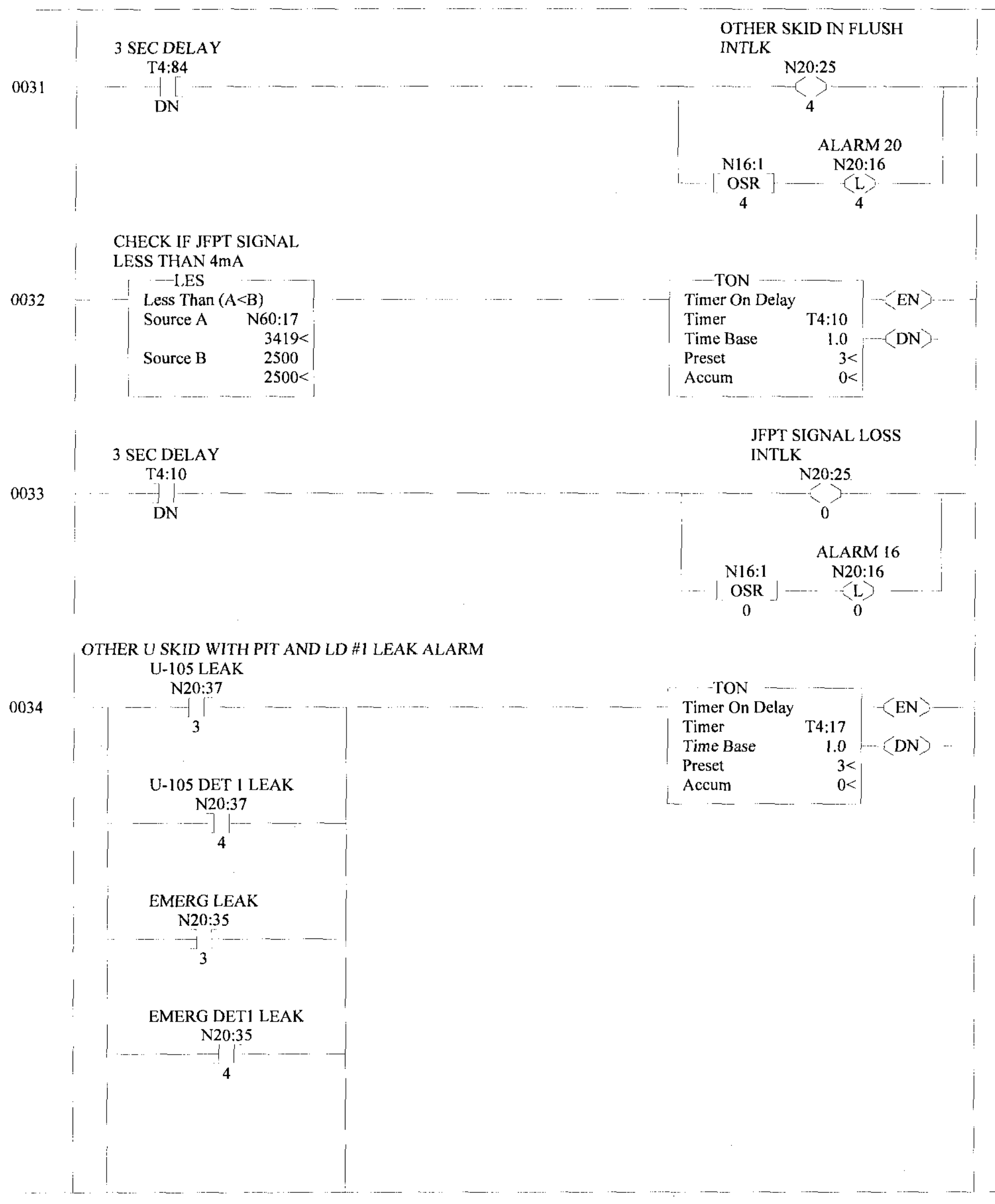


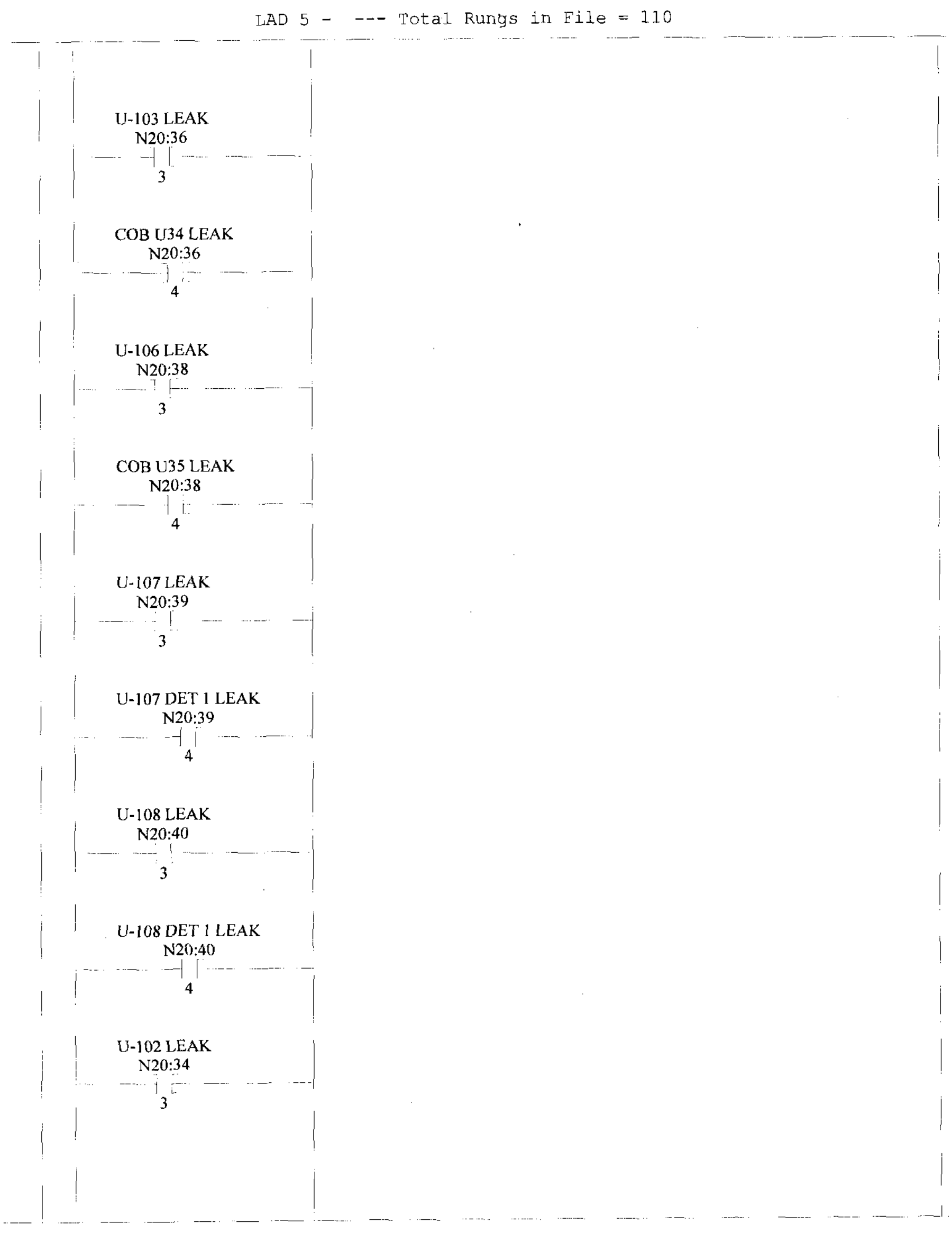




\section{LAD 5 - -- Total Rungs in File $=110$}
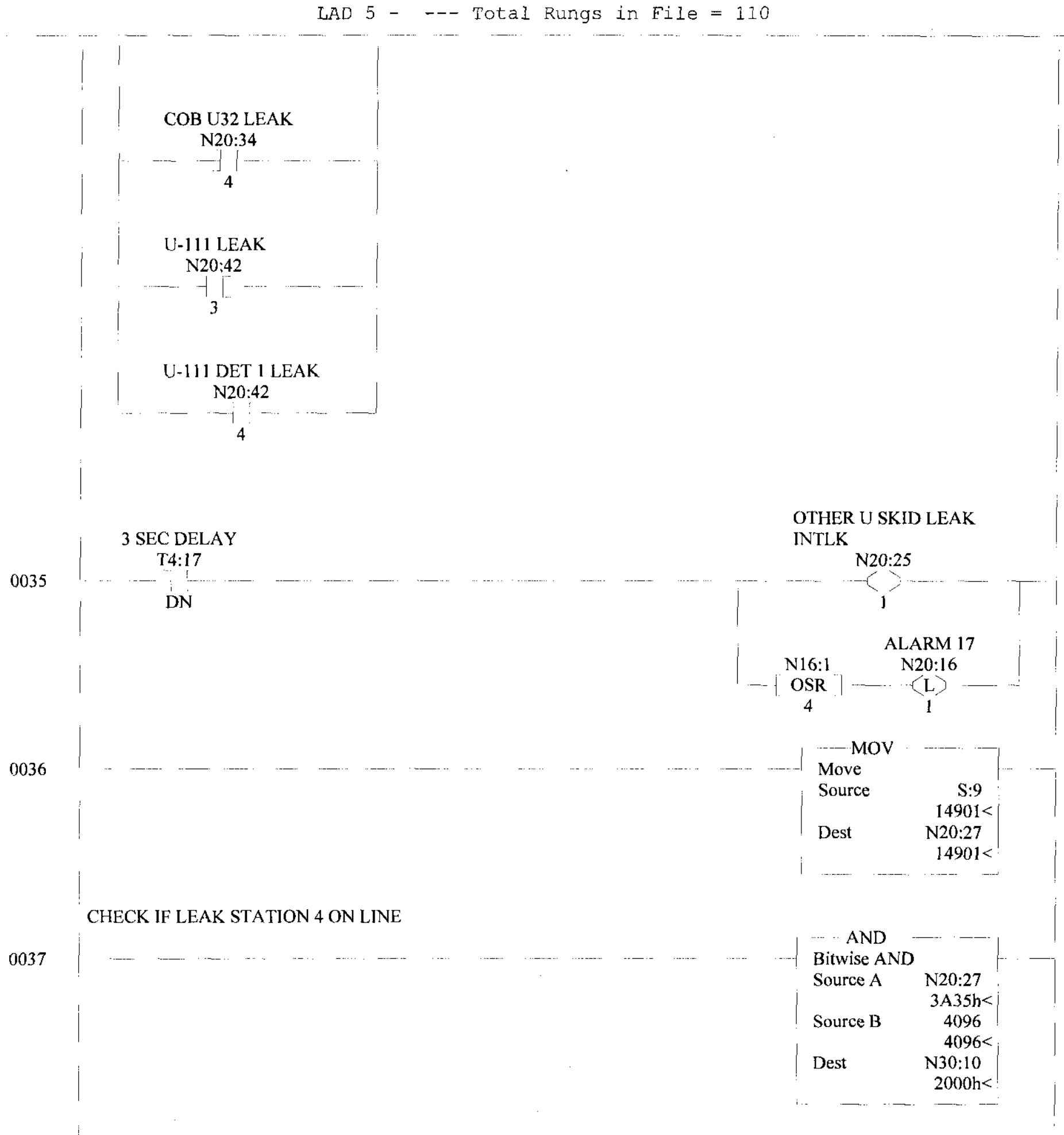
LAD 5 - -- Total Rungs in File $=110$

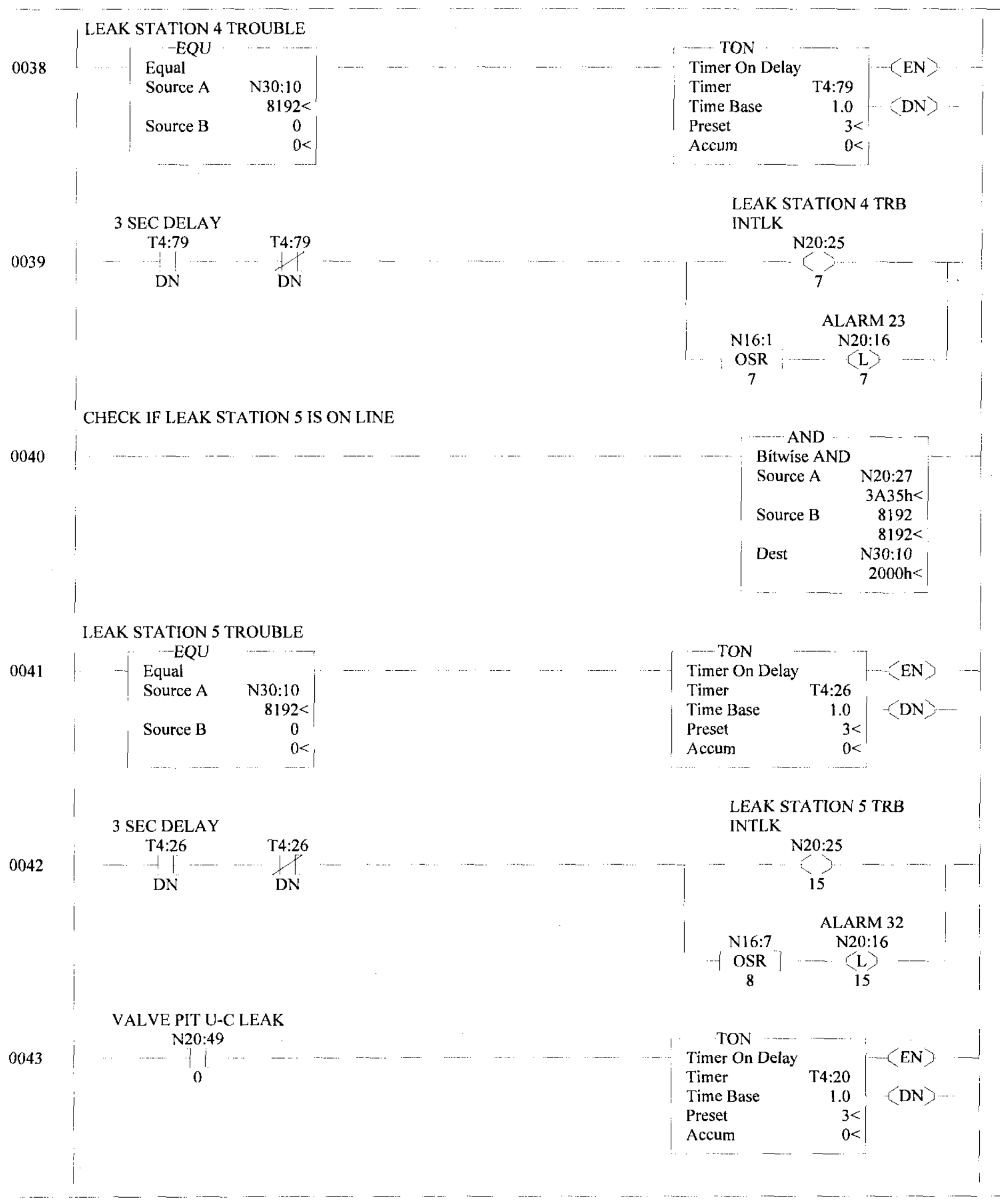


LAD 5 - -- Total Rungs in File $=110$

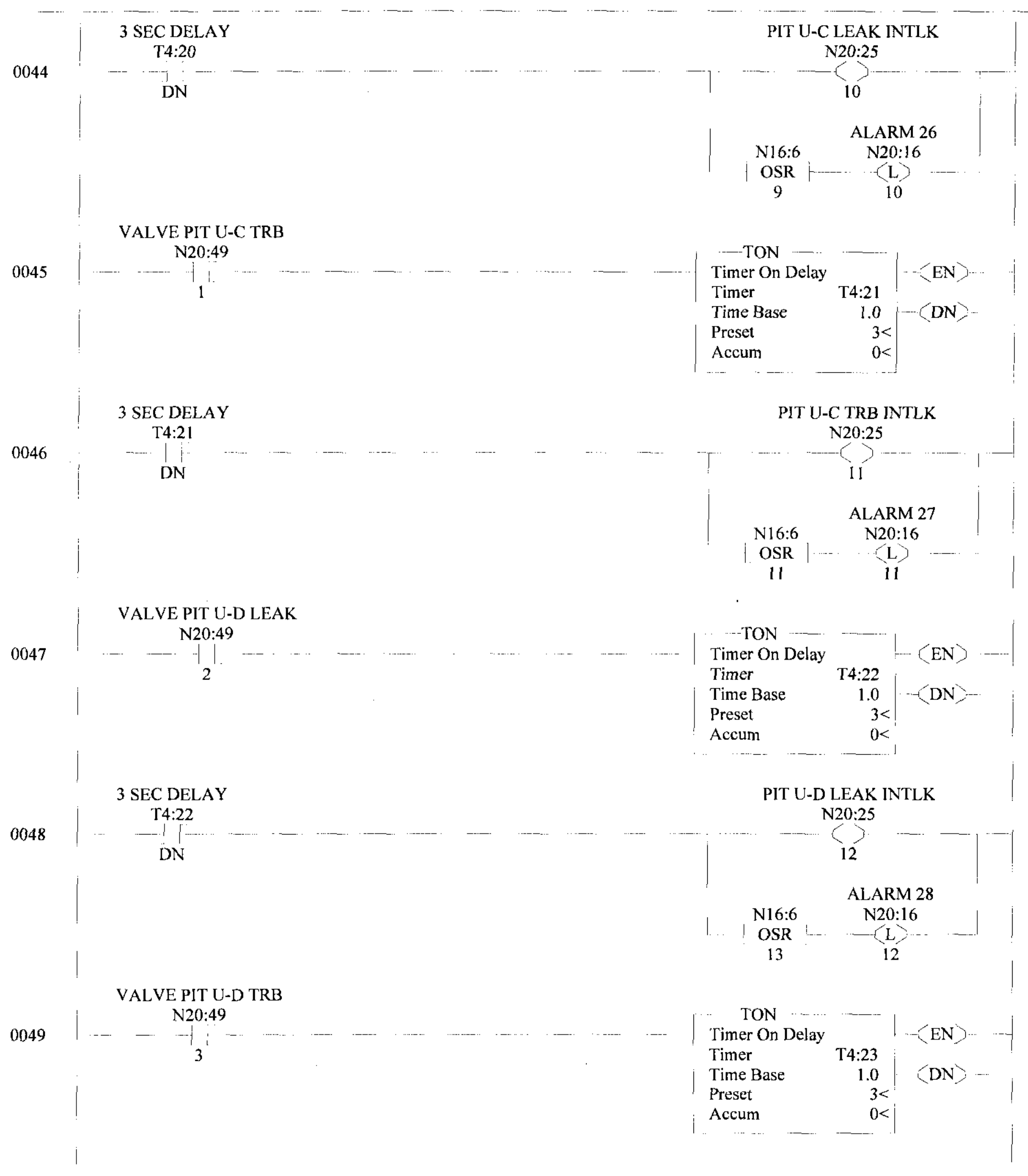


LAD 5 - -- Total Rungs in File $=110$

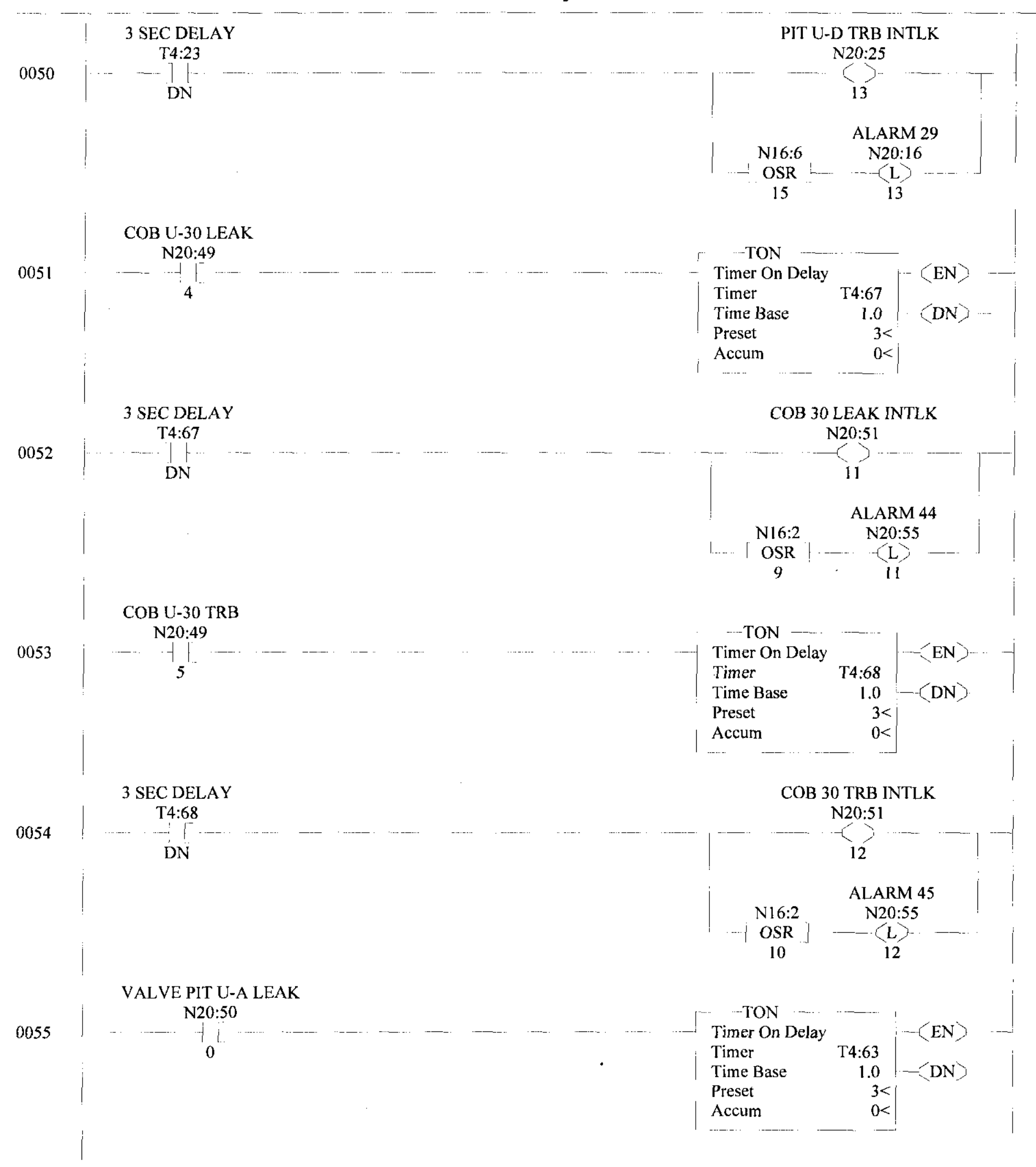


LAD 5 - -- Total Rungs in Fíle = 110

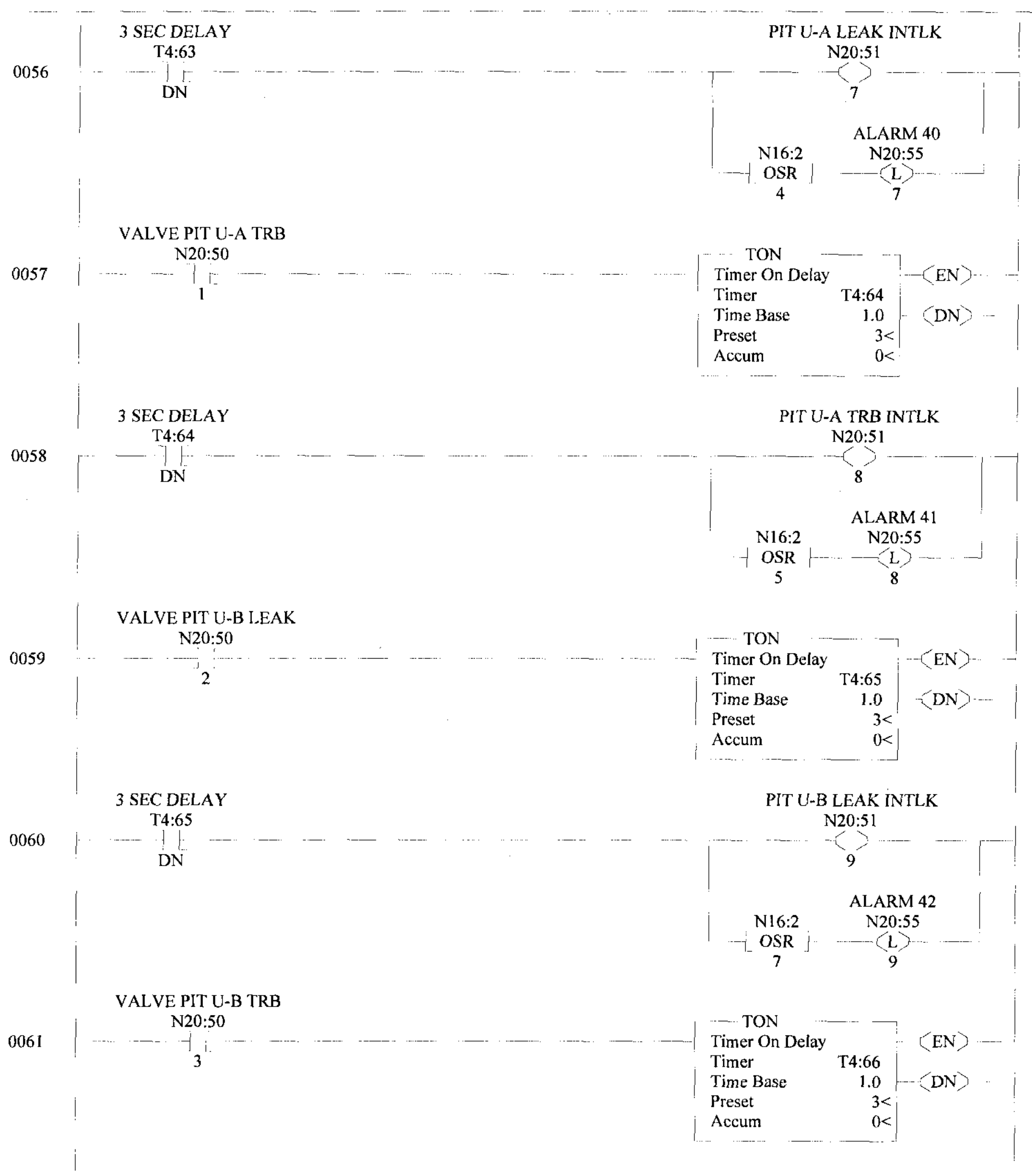




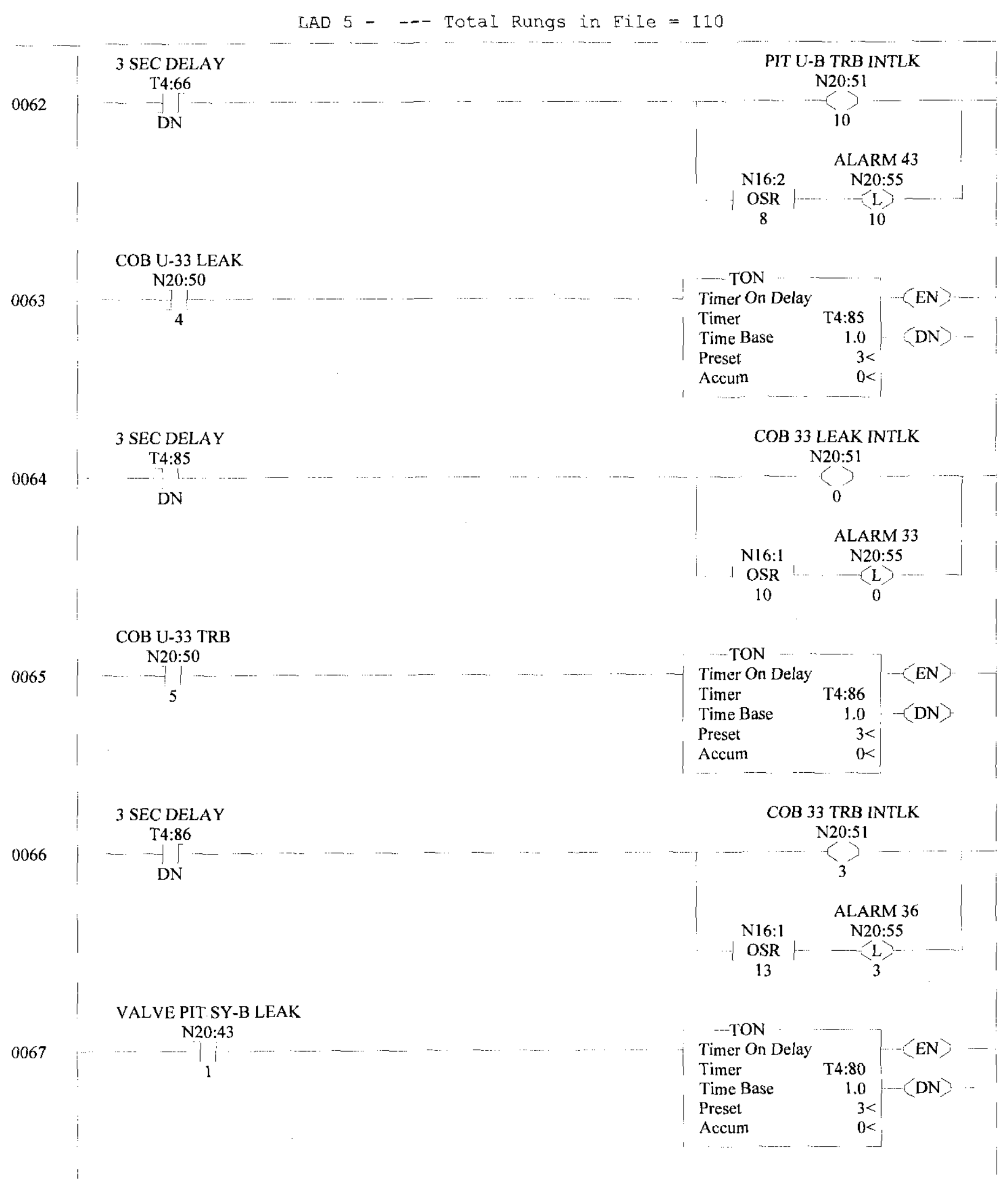


LAD 5 - - Total Rungs in File $=110$

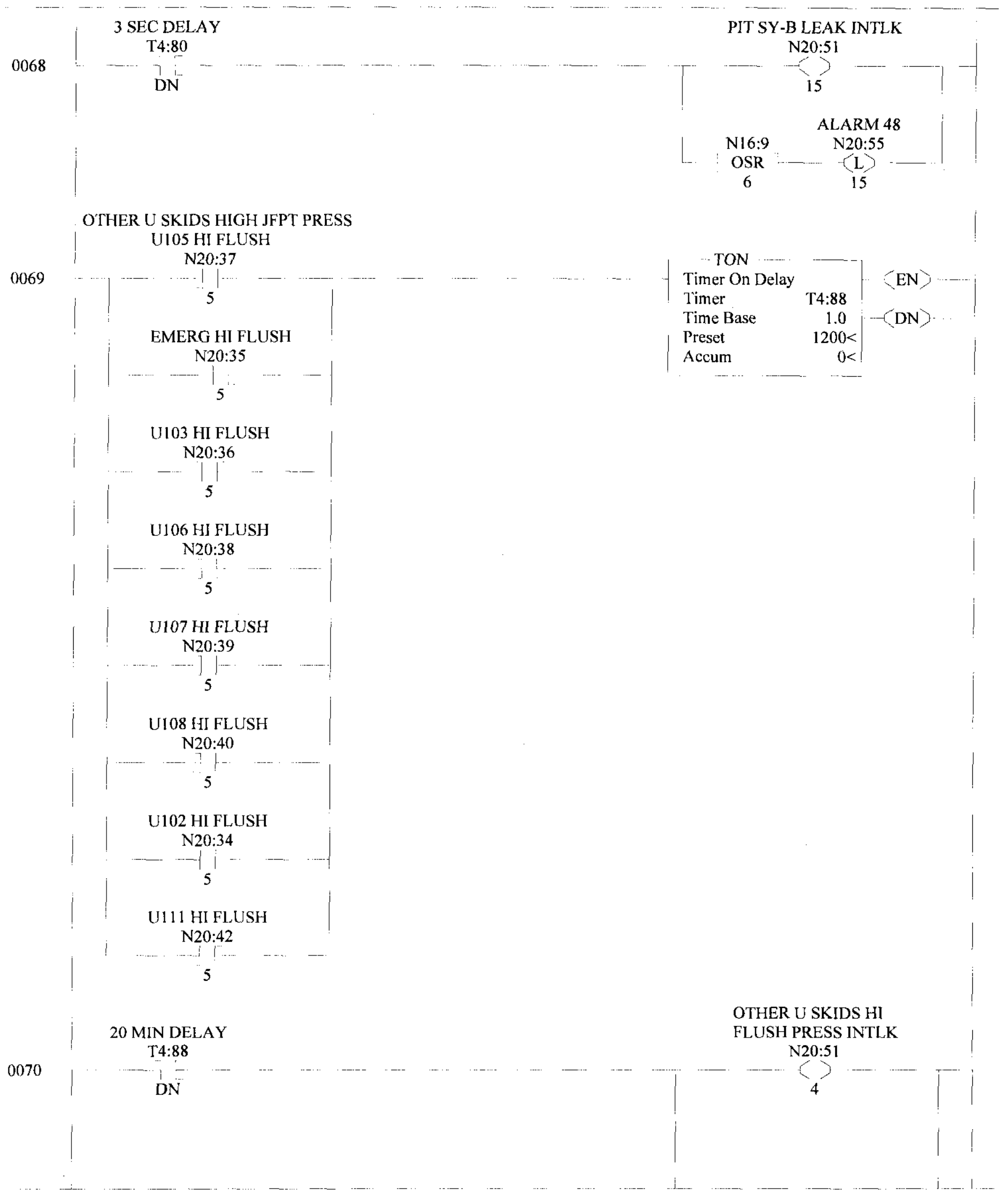


DN
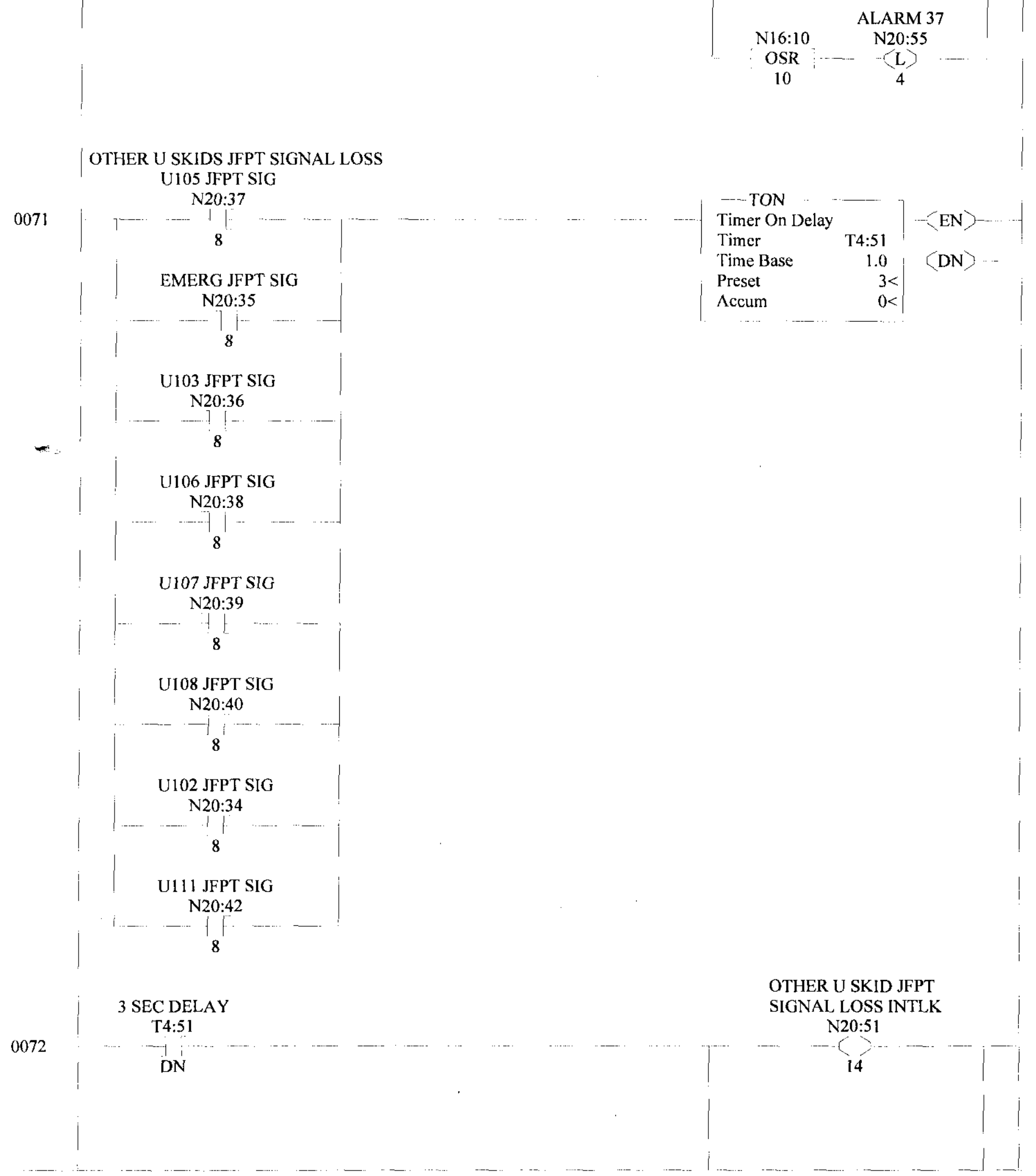
DN

$$
\text { U109NEW1RI PIC SKID N I }
$$

LAD 5 - -- Total Rungs in File $=110$

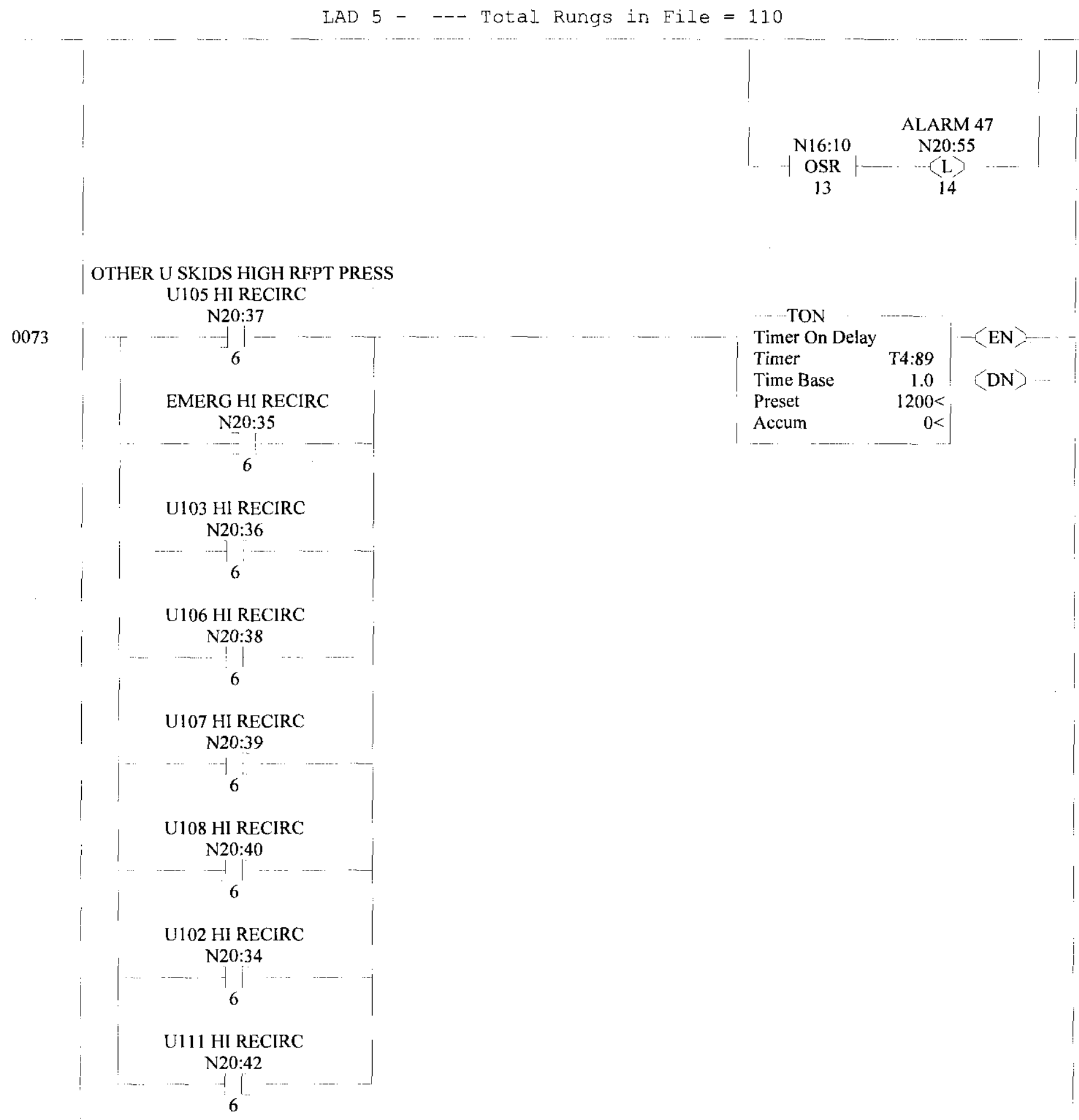

20 MIN DELAY

0074

$$
\mathrm{T} 4: 89
$$$$
\text { T4:89 }
$$

DN
OTHER U SKID HIGH RFPT PRESS INTLK 
DN

LAD 5 - -- Total Rungs in File = 110

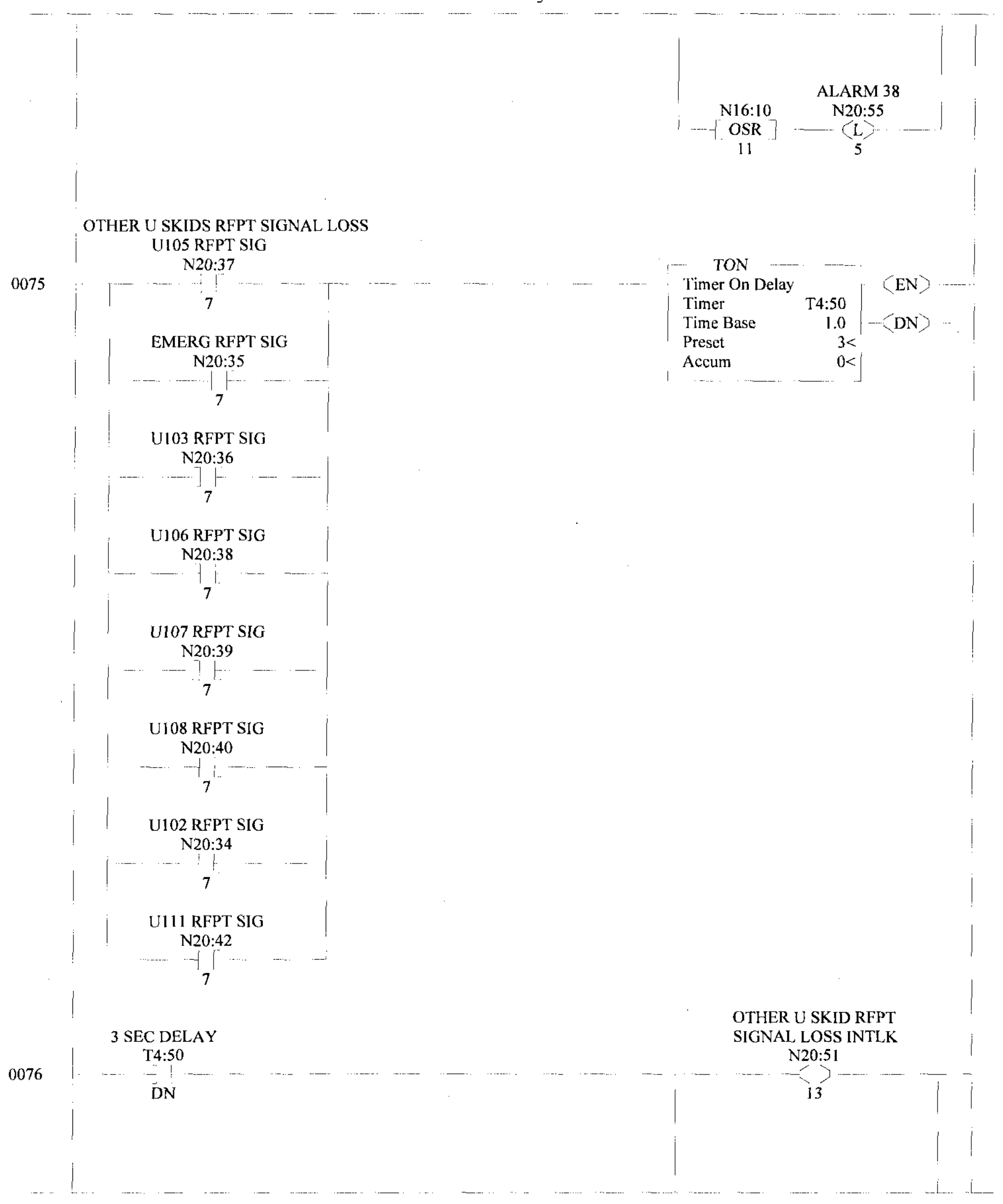


LAD 5 - -- Total Rungs in File $=110$
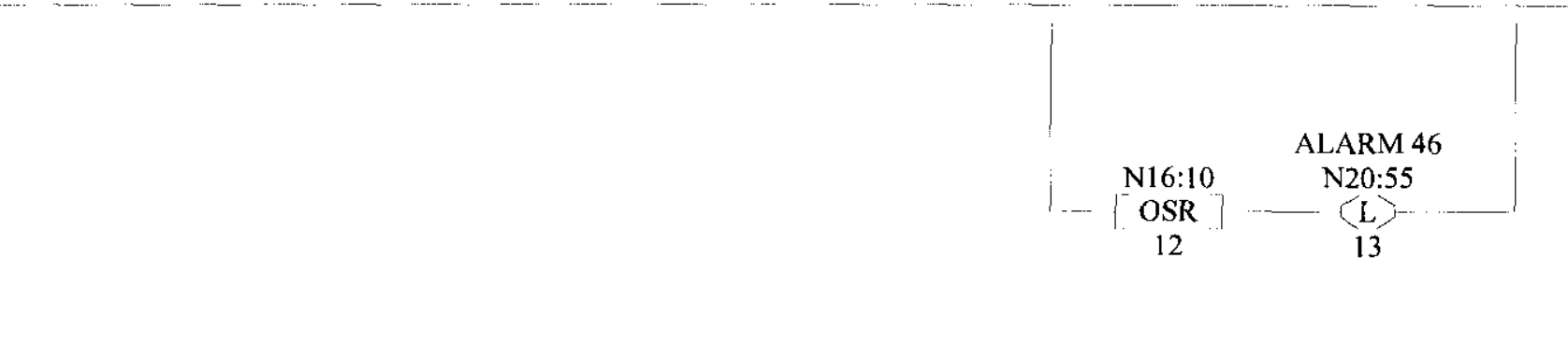


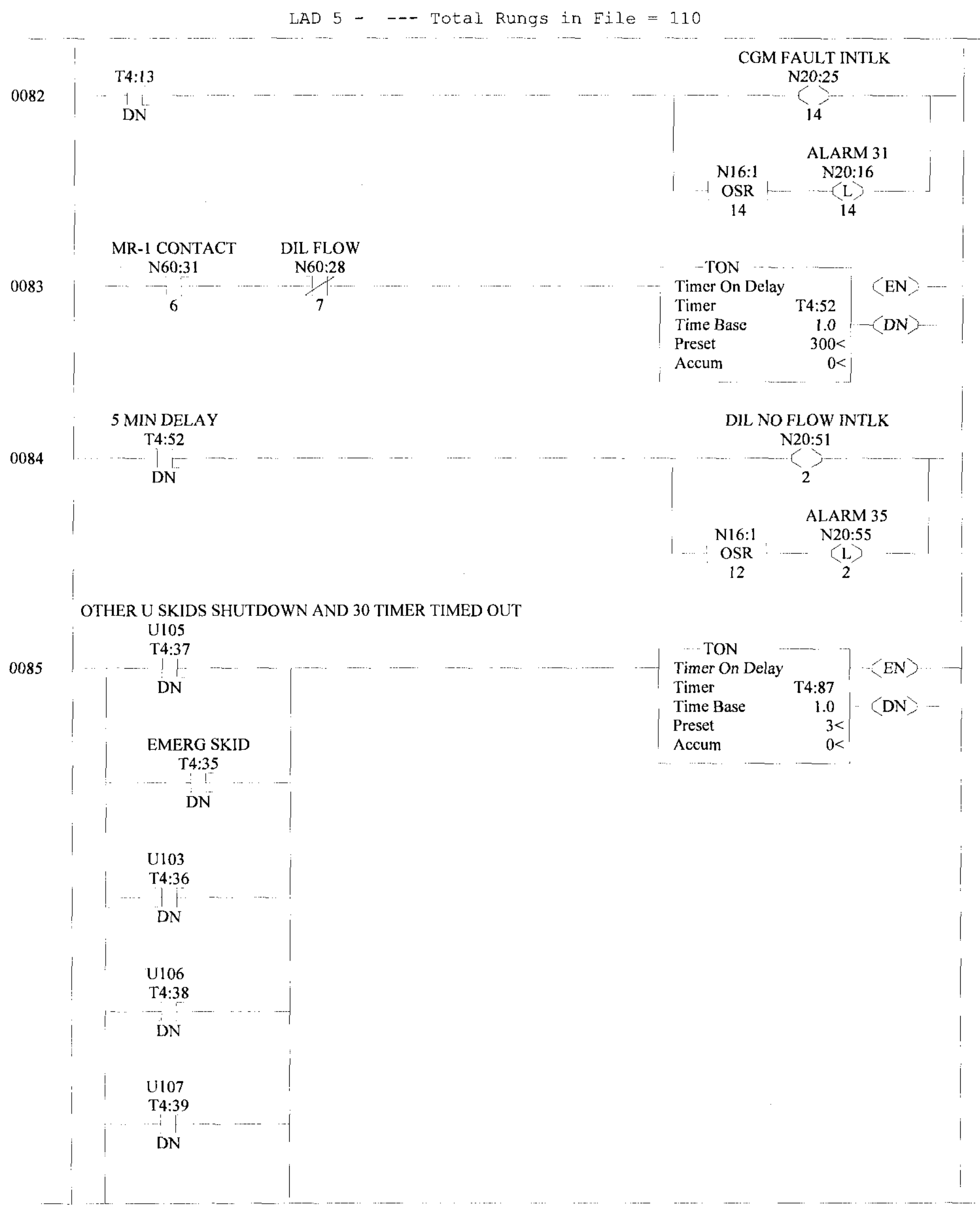




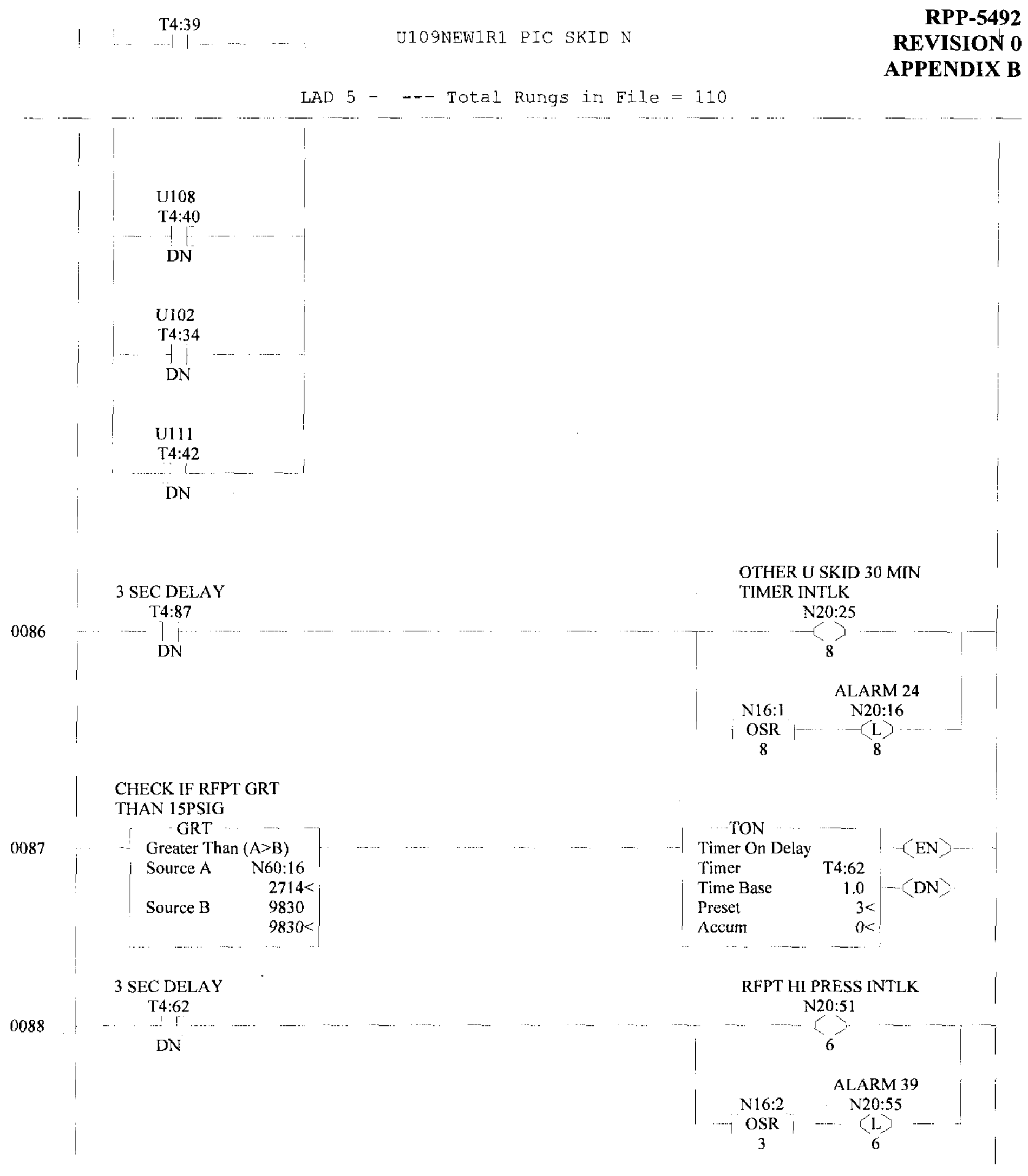

08:14:19 PAGE 23 wednesday, March 15, 2008AGE B 3\$ OF B 90 
LAD 5 - -- Total Rungs in File $=110$

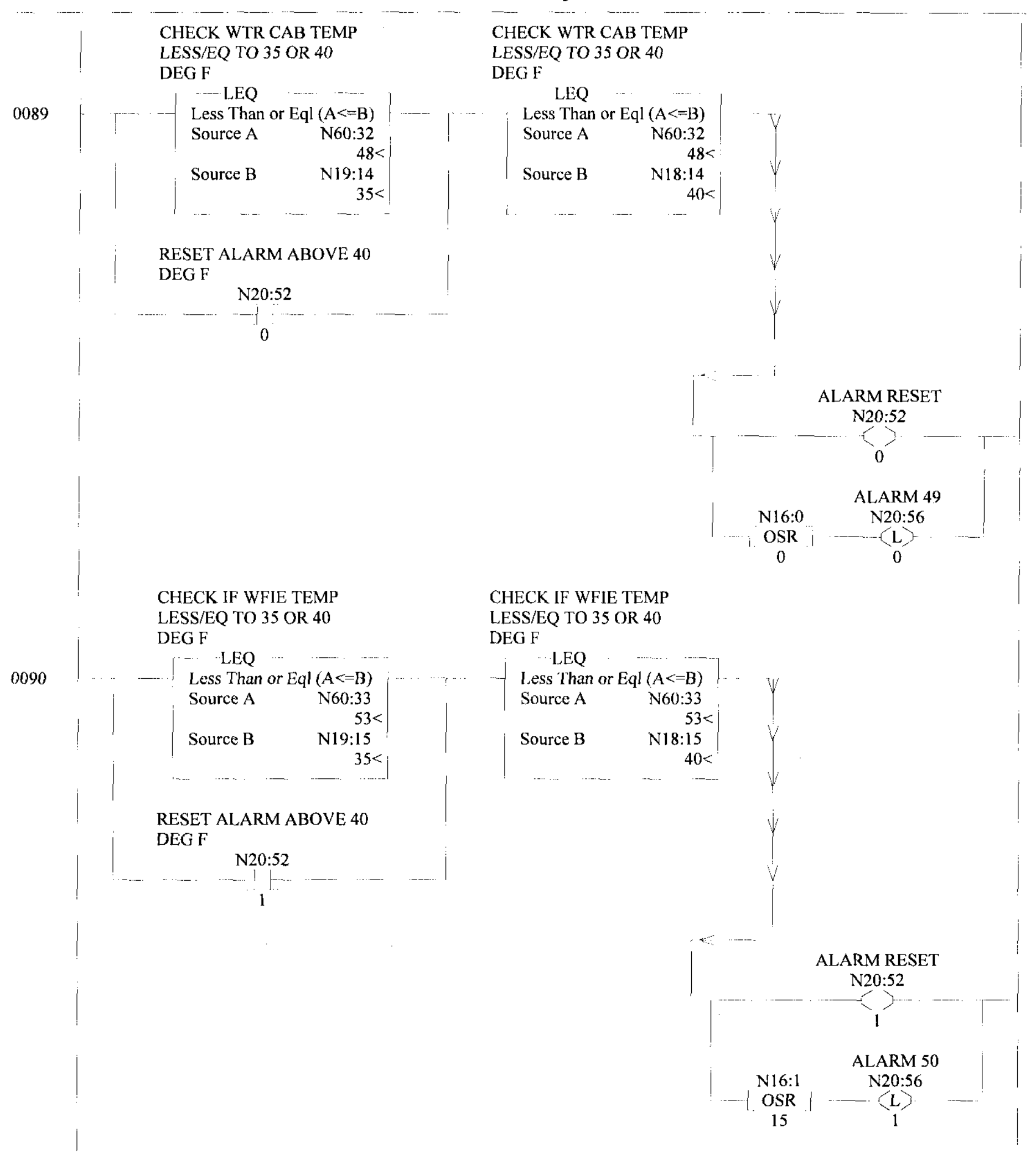


0091
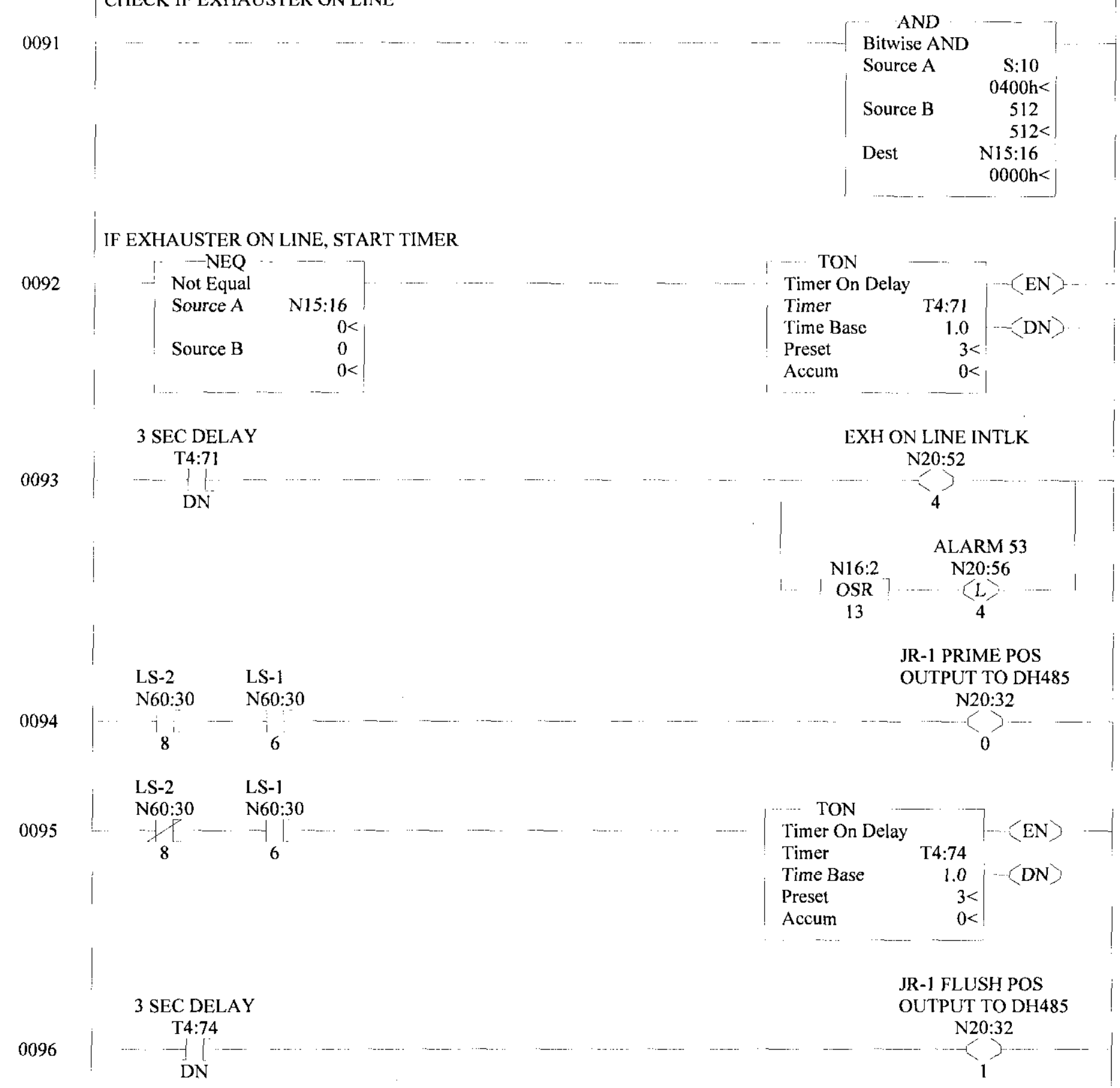

MR-1 CONTACT

JR-1 FLUSH POS OUTPUT TO DH485 
RPP-5492

LAD 5 - -- Total Rungs in File $=110$
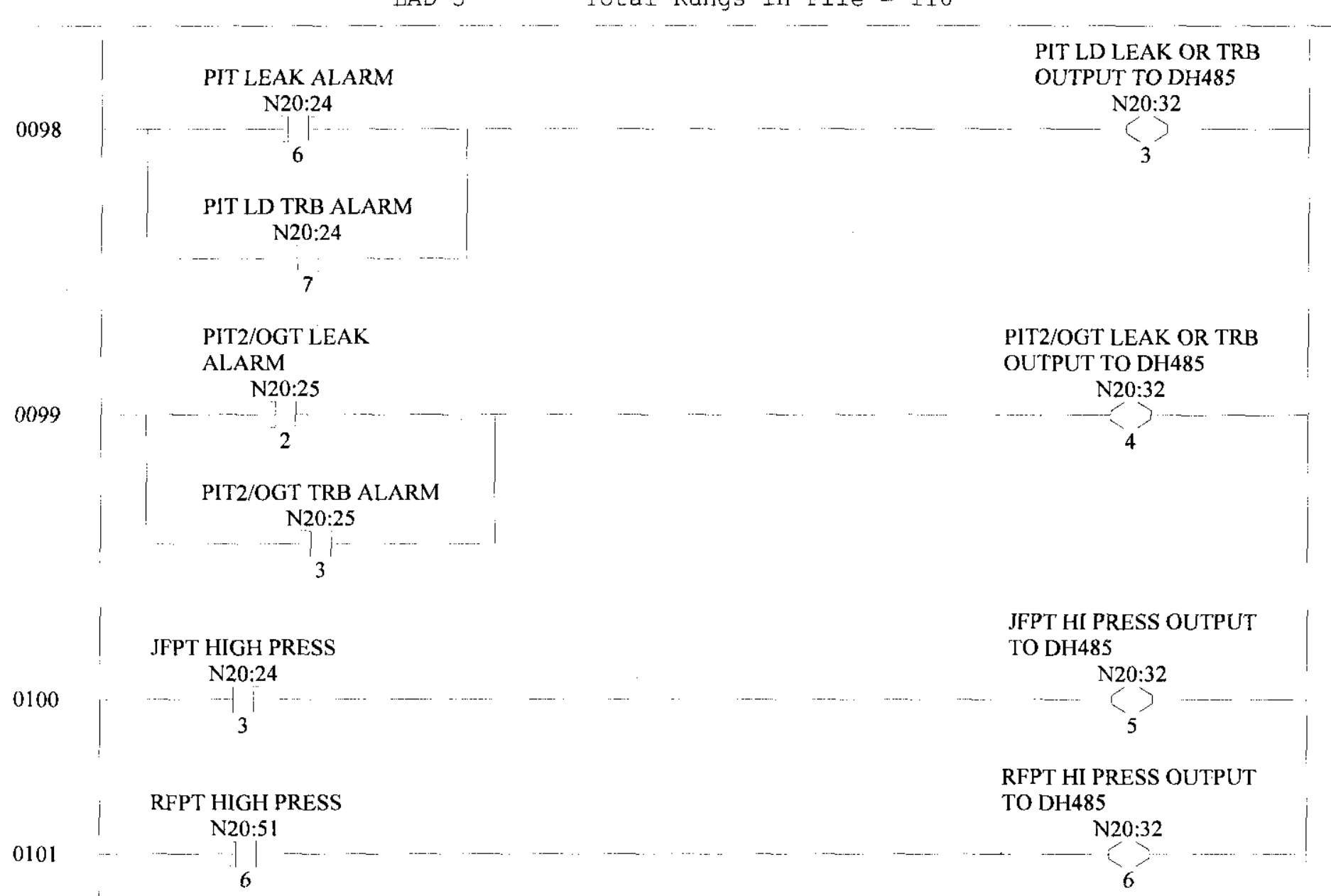

JFPT HI PRESS OUTPUT TO DH485

$\mathrm{N} 20: 32$

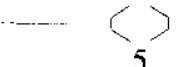

RFPT HI PRESS OUTPUT TO DH485 $\mathrm{N} 20: 32$<smiles>C1PC2CC12</smiles>

RFPT SIG LOSS OUTPUT TO DH485 $\mathrm{N} 20: 32$

0102

RFPT SIG LOSS ALARM $\mathrm{N} 20: 24$

14

JFPT SIG LOSS ALARM N20:25

0103

$$
0
$$

INPUT TO WONDERWARE

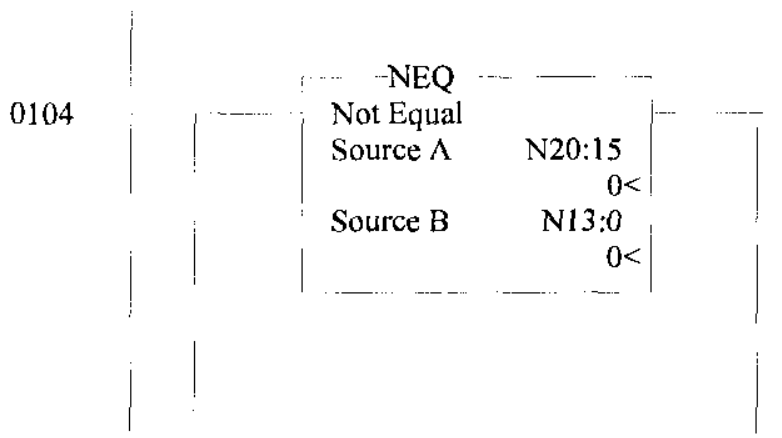

JFPT SIG LOSS OUTPUT TO DH485

$$
8
$$

FLASH FOR ALARM CONDITIONS

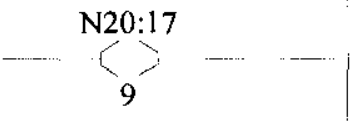


LAD 5 - -- Total Rungs in File $=110$

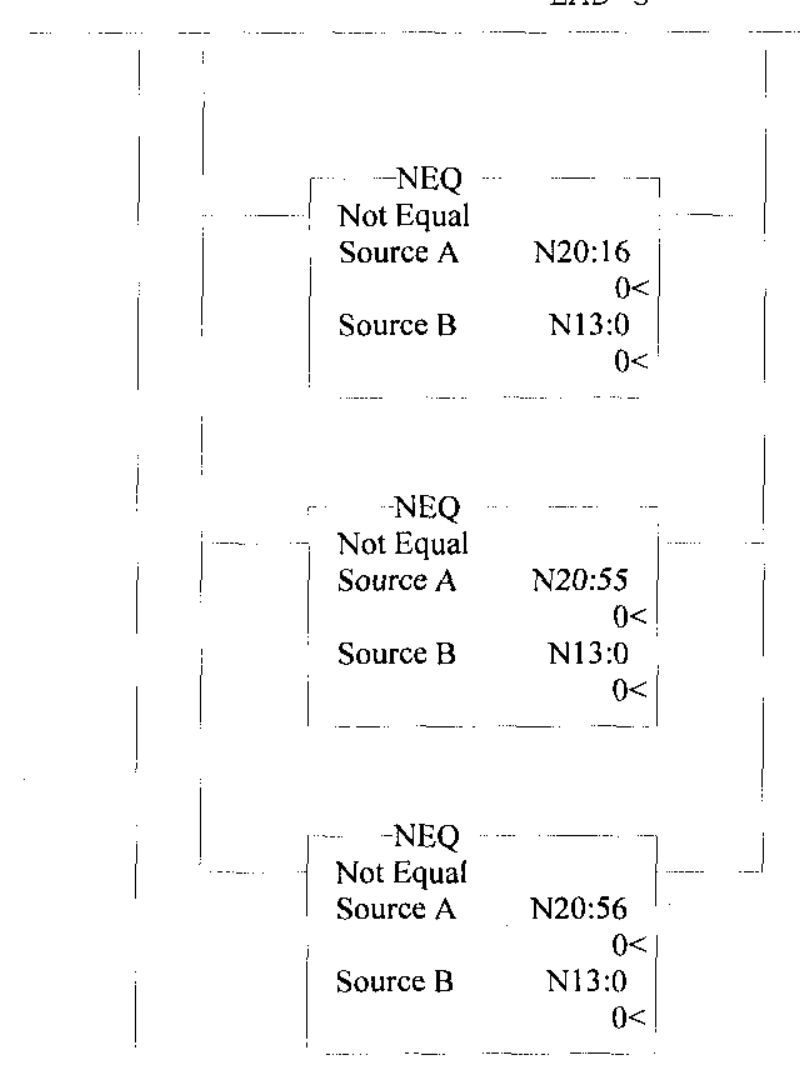

INPUT TO WONDERWARE

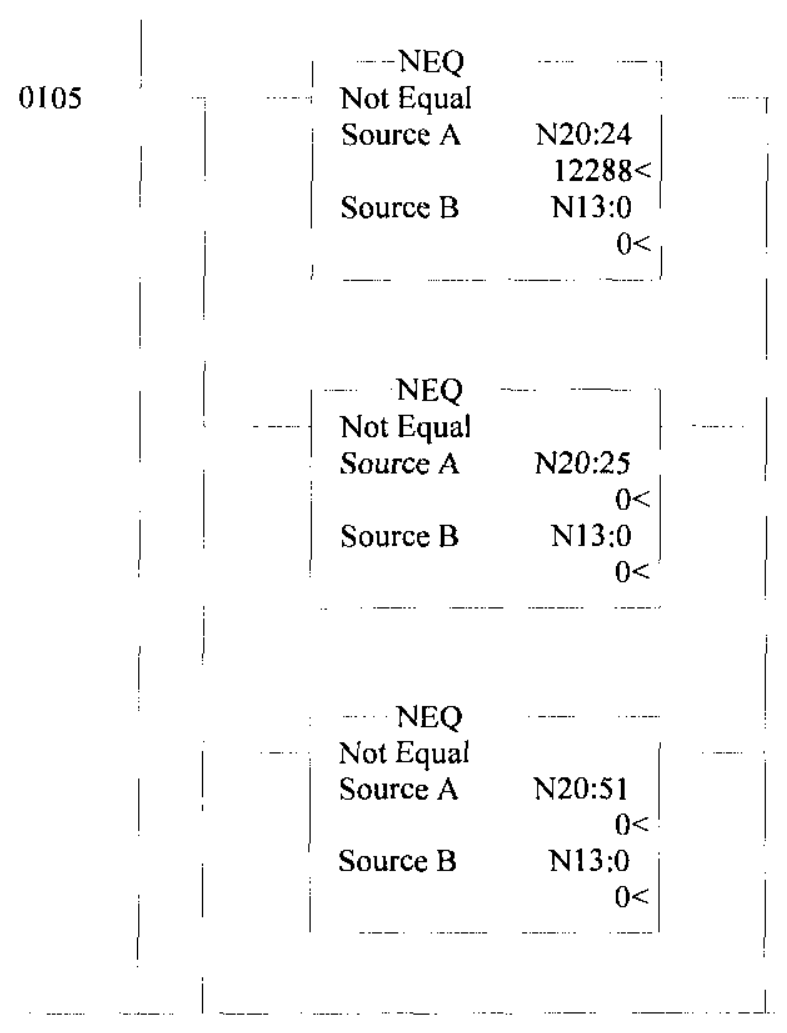

COLOR CHANGE FOR ALARM CONDITIONS 


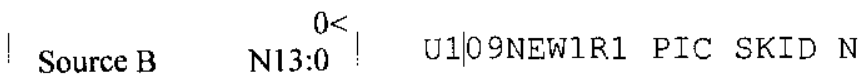

LAD 5 - -- Total Rungs in File $=110$
RPP-5492

REVISION 0

APPENDIX B

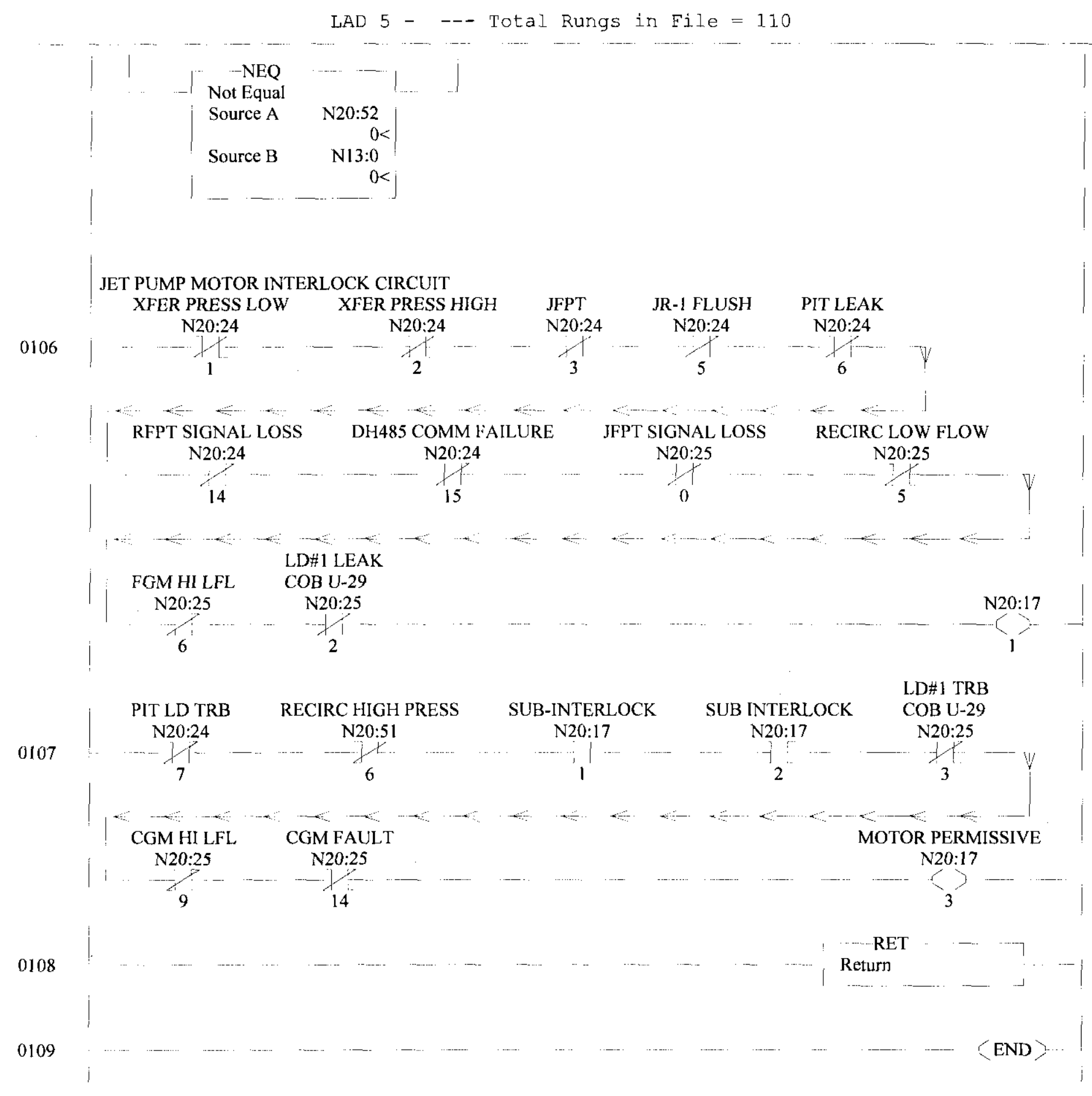

08:14:19 PAGE 28 wednesday, March 15, 2008AGE B 39 OF B 90 


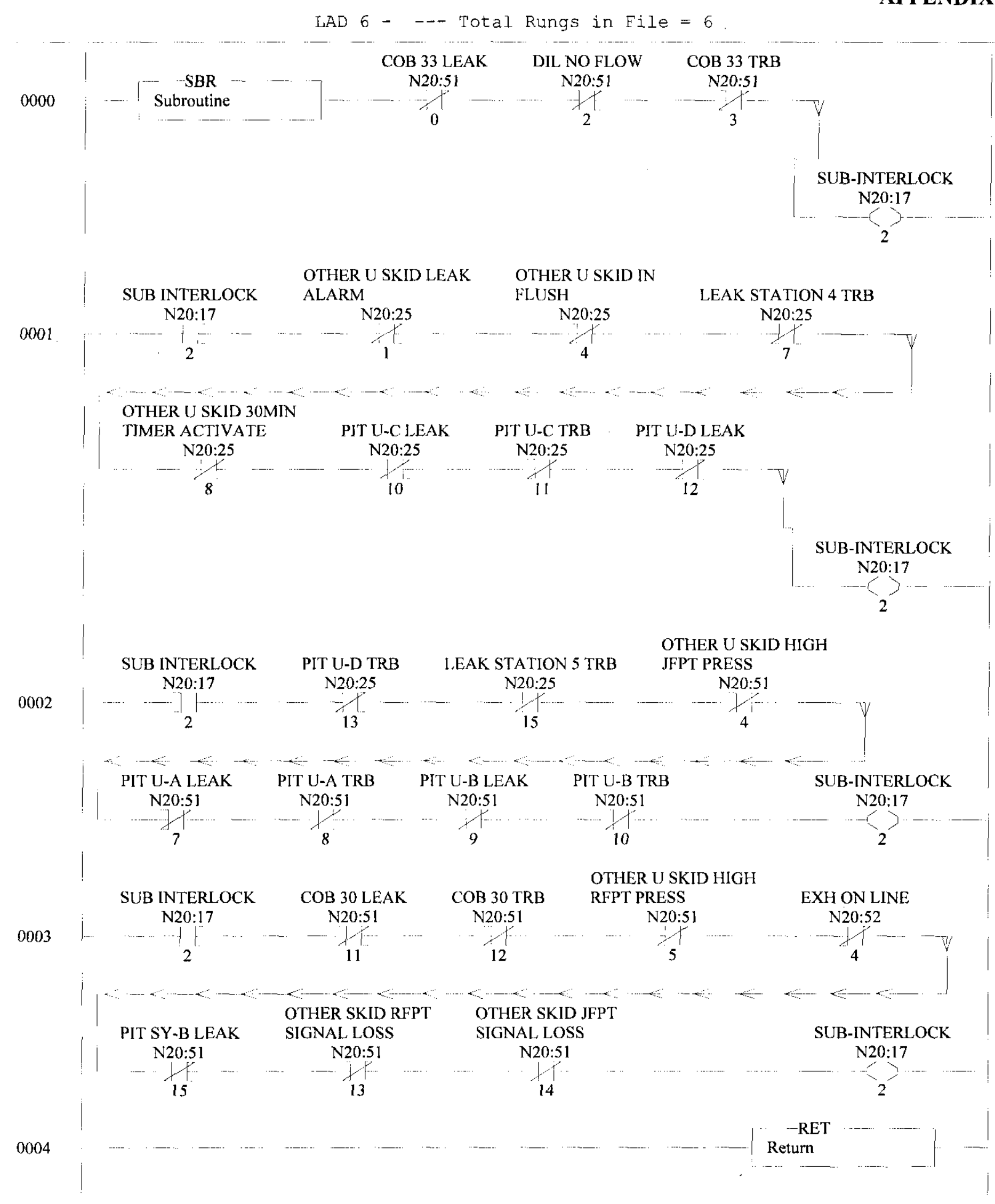


LAD 6 - -- Total Rungs in File $=6$ 


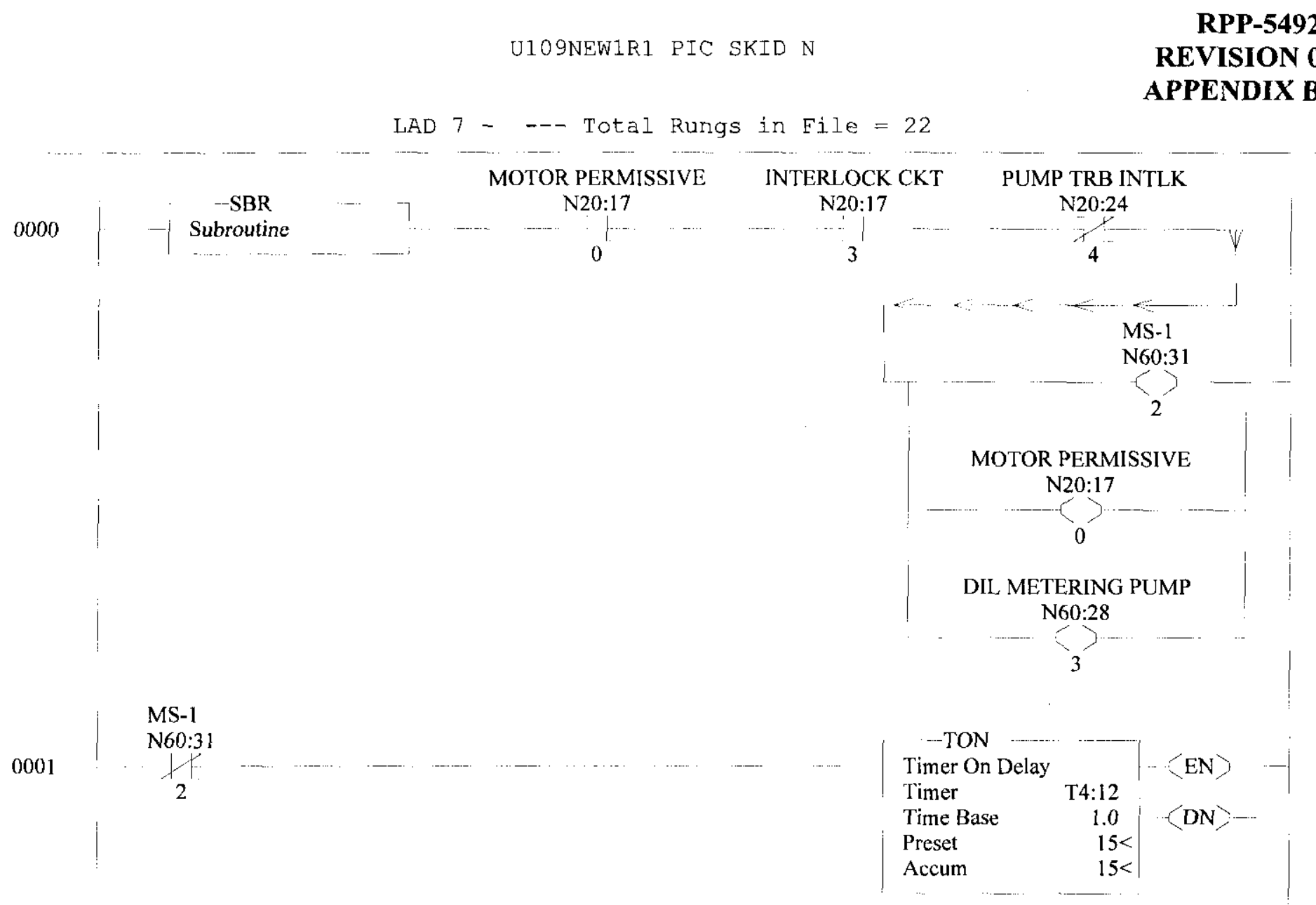

MS- 1

DIL CONTACTOR

$\mathrm{N} 60: 31$

0002

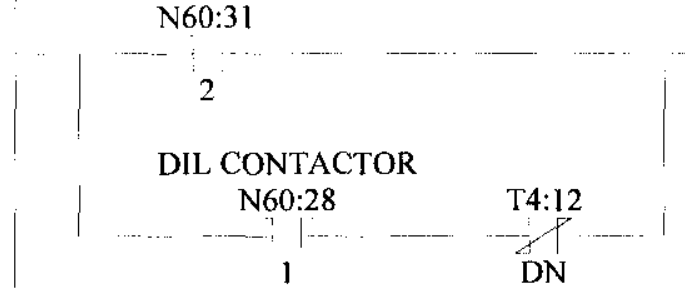

0003

MOTOR PERMISSIVE

$\begin{array}{cc}\mathrm{N} 20: 17 & \mathrm{~N} 16: 6 \\ 0 & \text { OSR } \\ 0 & 1\end{array}$

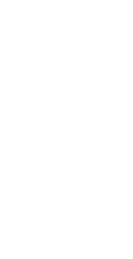

0004

$\mathrm{N} 20: 17$
$j$
7

-.... JSR

Jump To Subroutine

SBR File Number

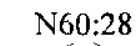


LAD 7 - - Total Rungs in File $=22$

0005
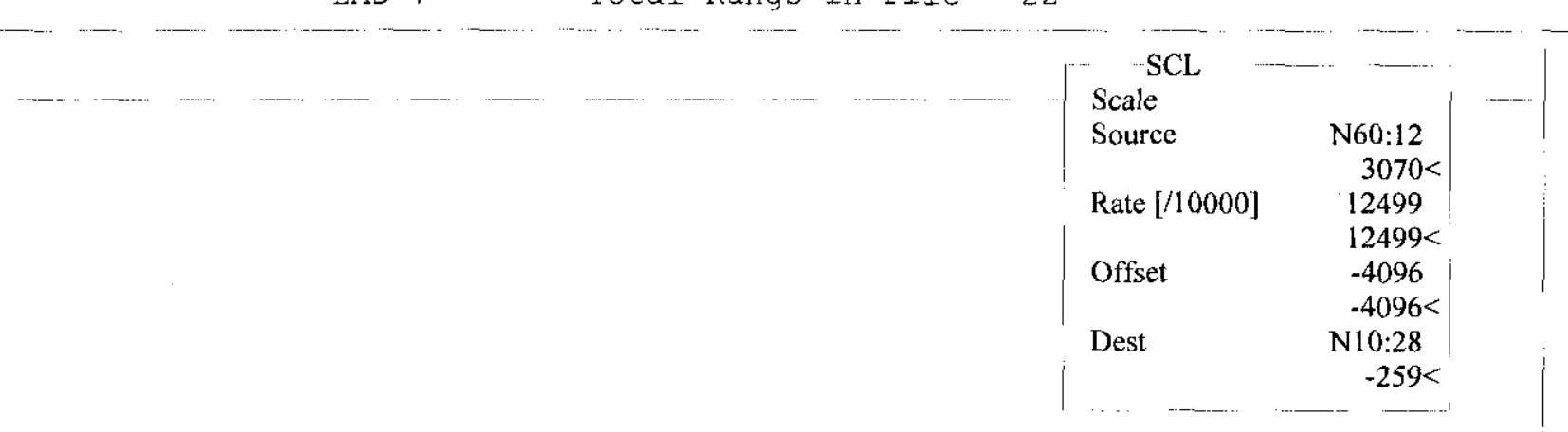

0006

SGT LOW

N20:24

0007

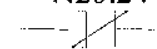

13

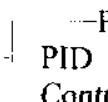

Control Block

Process Variable

Control Variable

Control Block Length
Setup Screen

N10:0

N10:28

N10:29

23

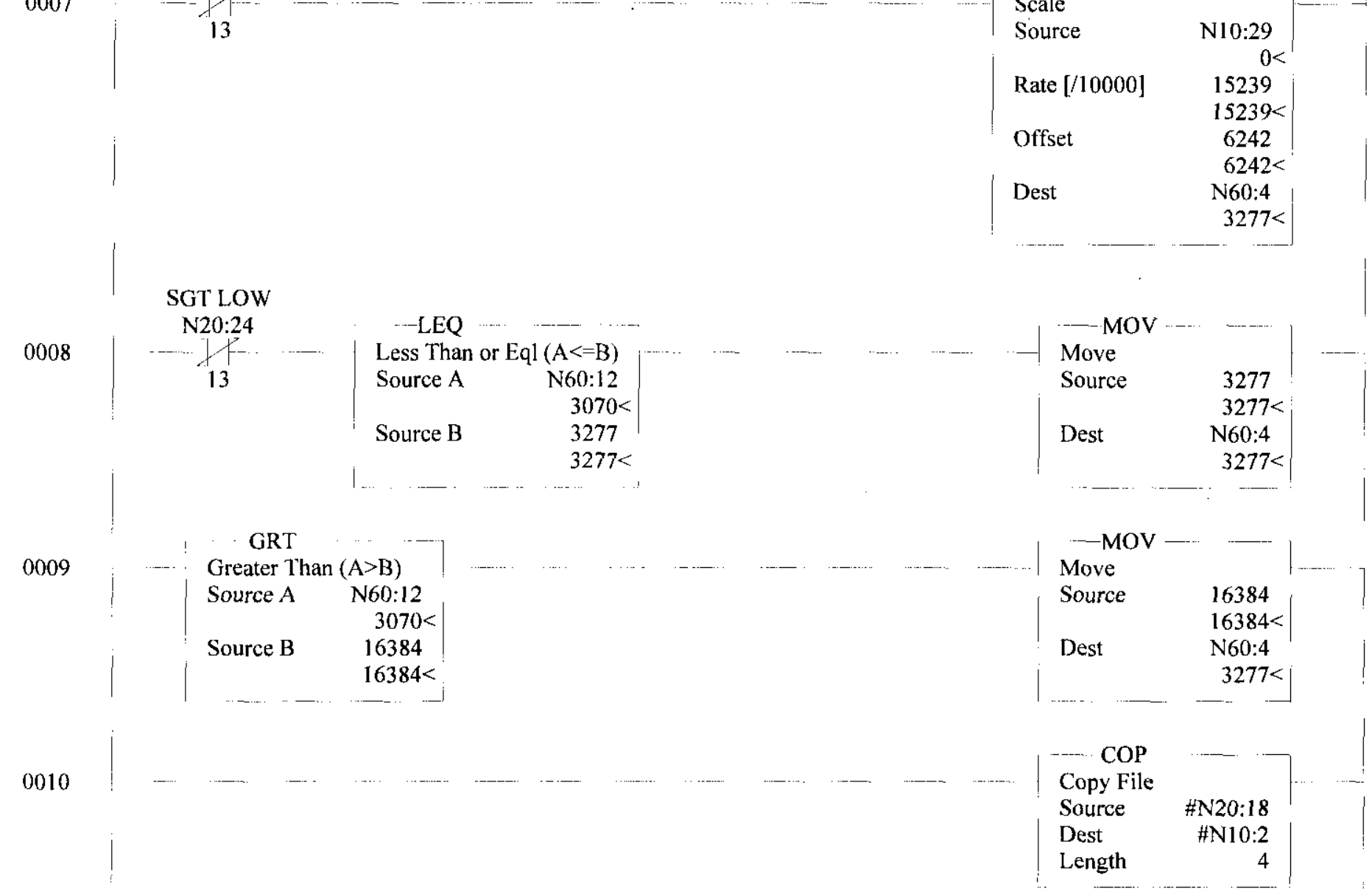


0011
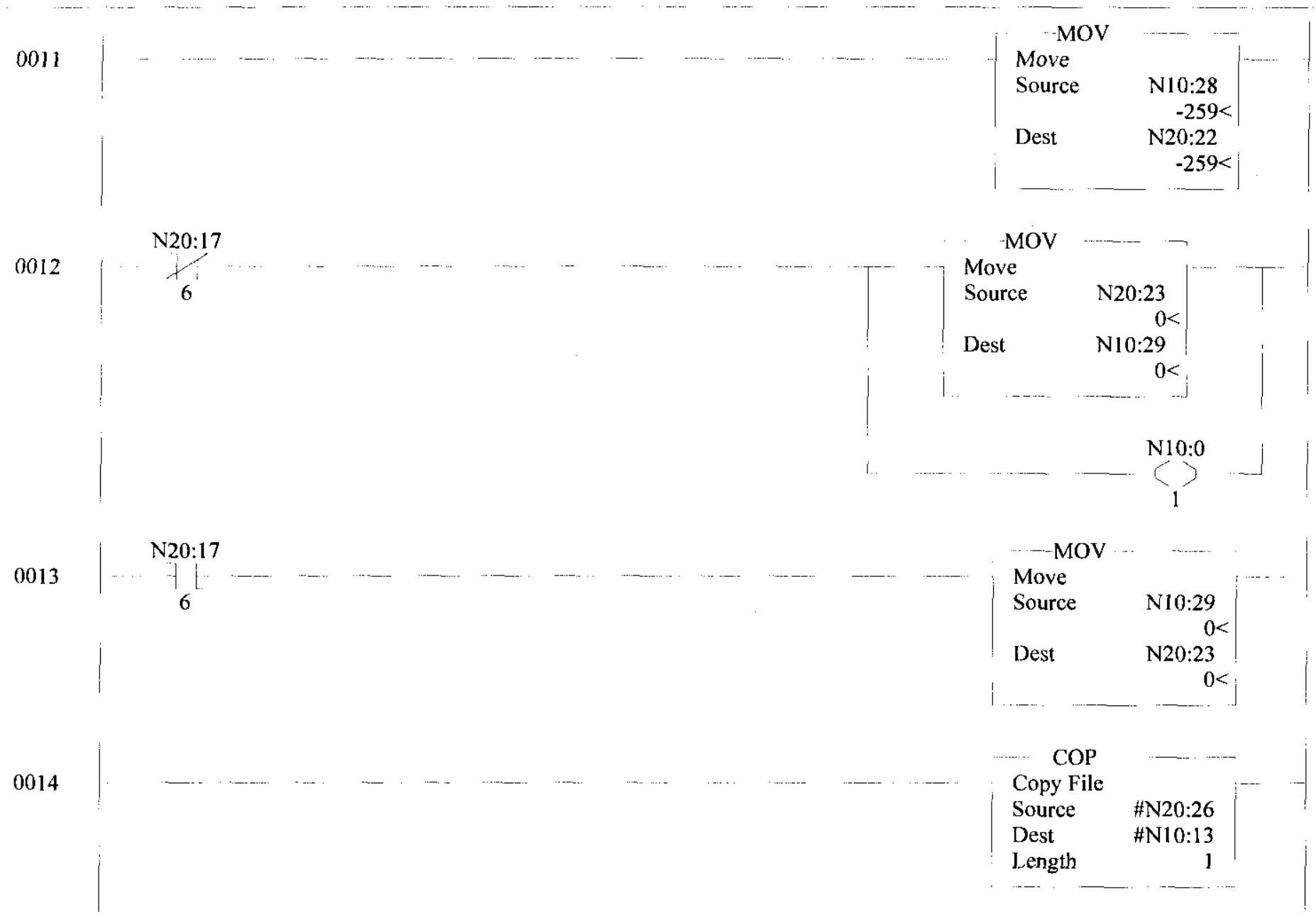

$\mathrm{N} 20: 17$
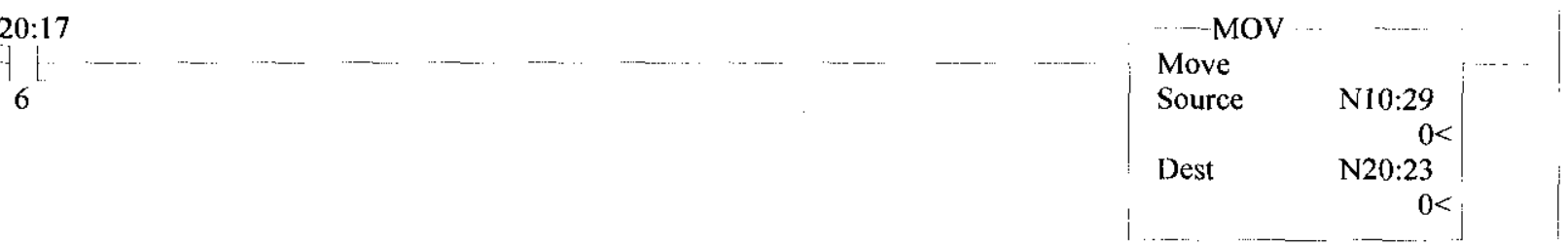

0015
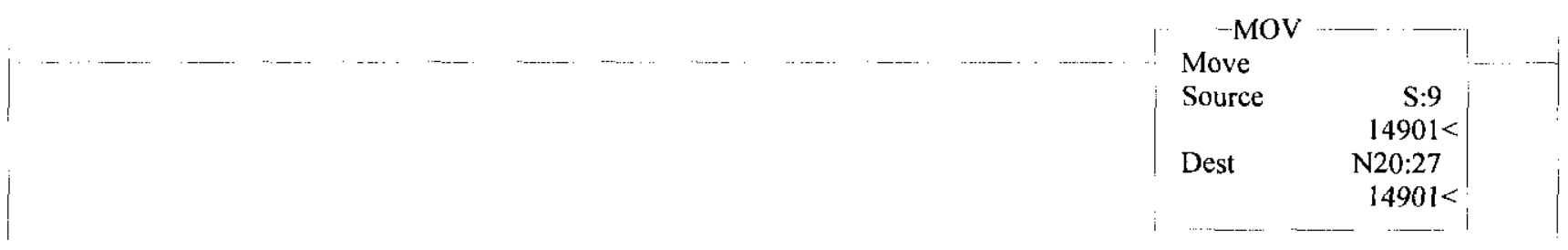

0016

0017

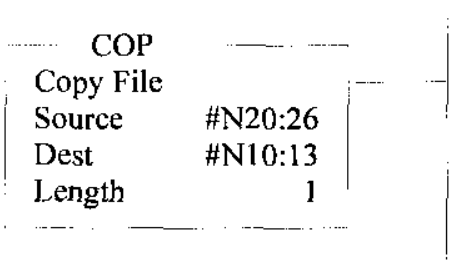

$-\mathrm{MOV}$

Move

Source N20:28

Dest

$14388<$

N15:0

$14388<$

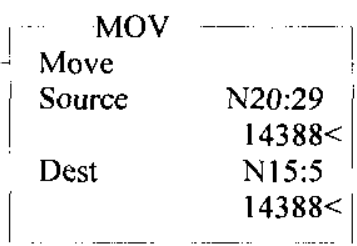




\section{U109NEW1RI PIC SKID N}

LAD 7 - - Total Rungs in File $=22$

0018

0019

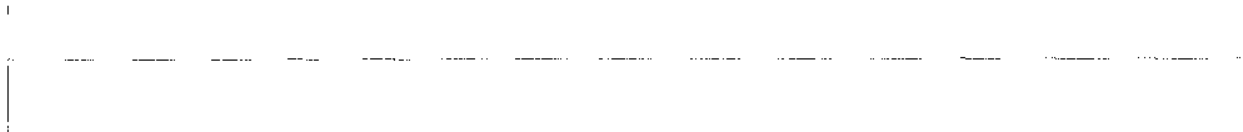

(2)

0020

0021
RPP-5492

REVISION 0

APPENDIX B
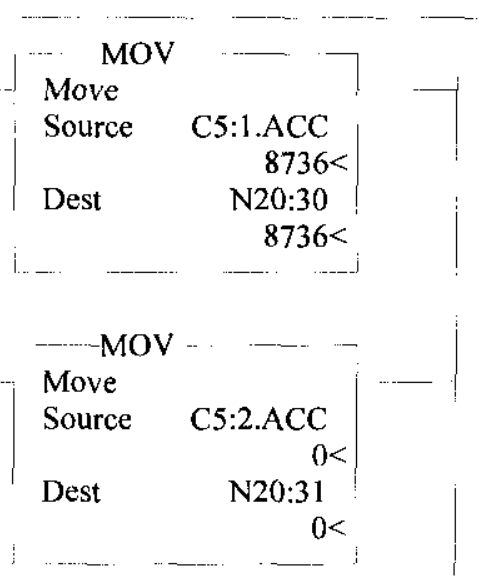

Return

08:14:20 PAGE 4 wednesday, March 15, 2000PAGE B 45 OF B 90 


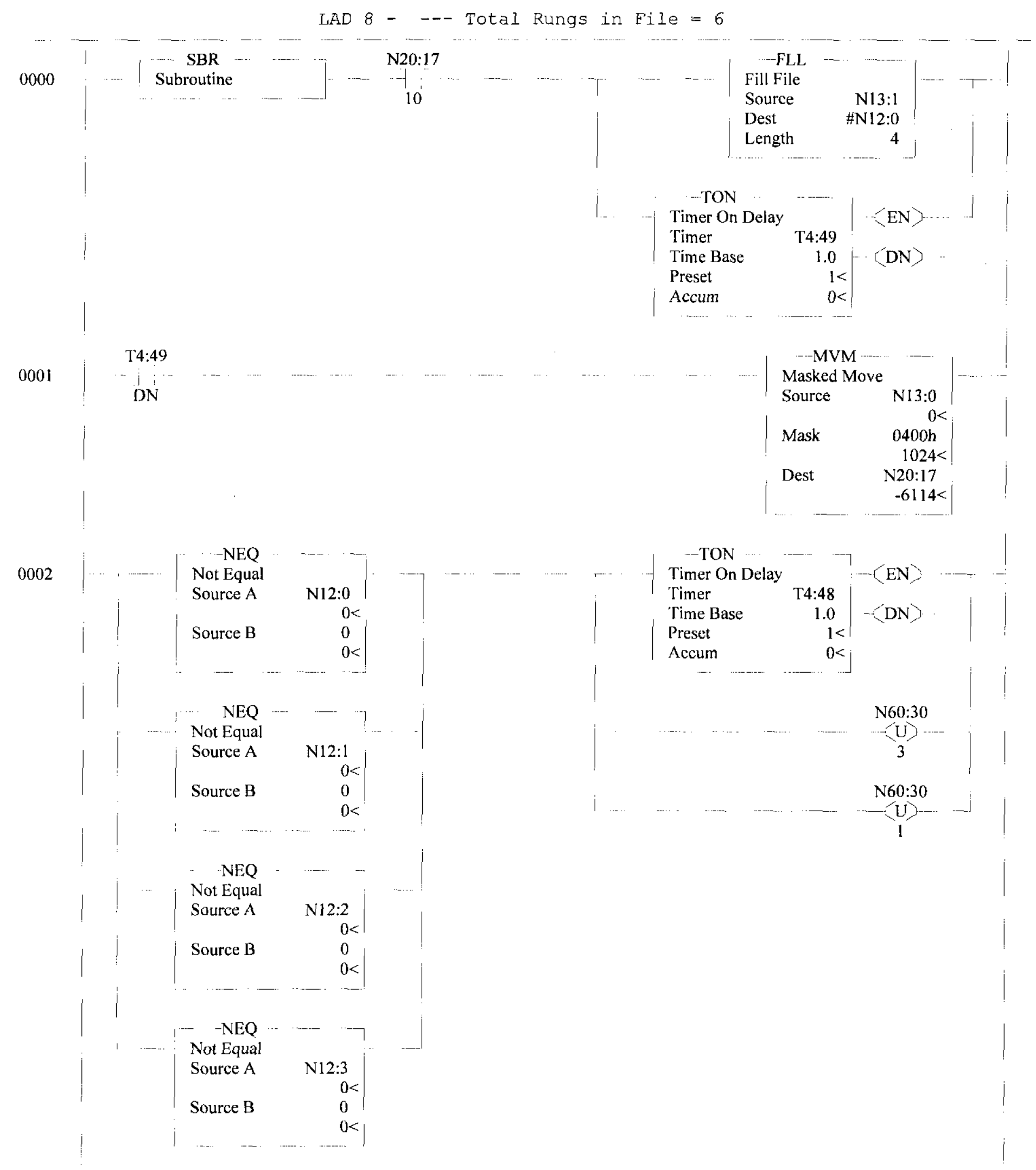


RPP-5492

LAD 8 - - - Total Rungs in File $=6$

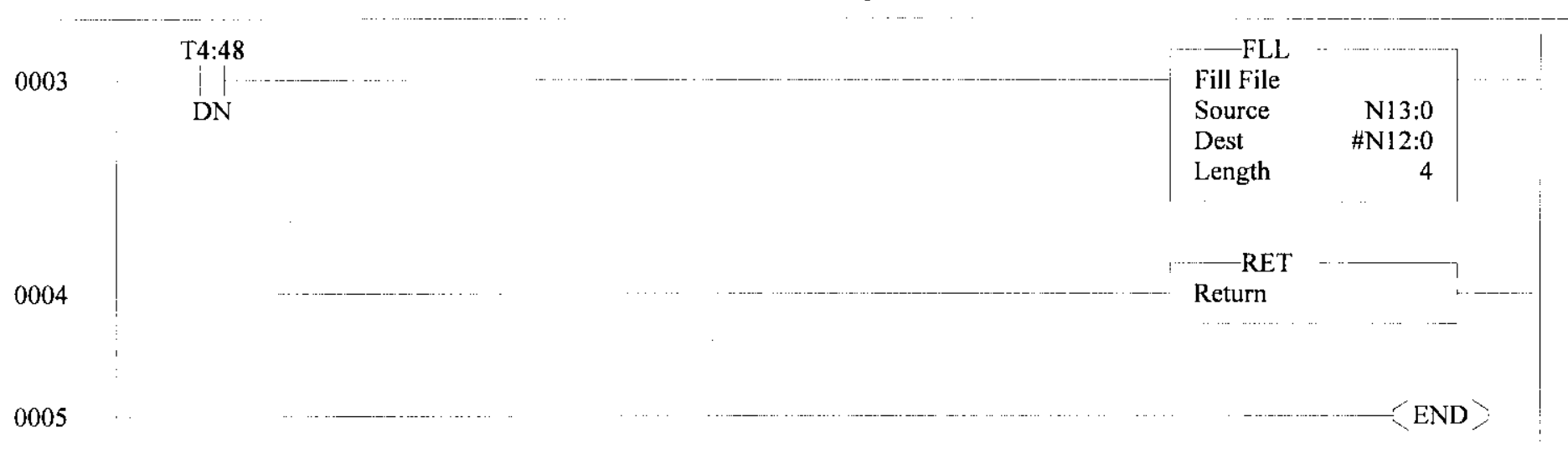




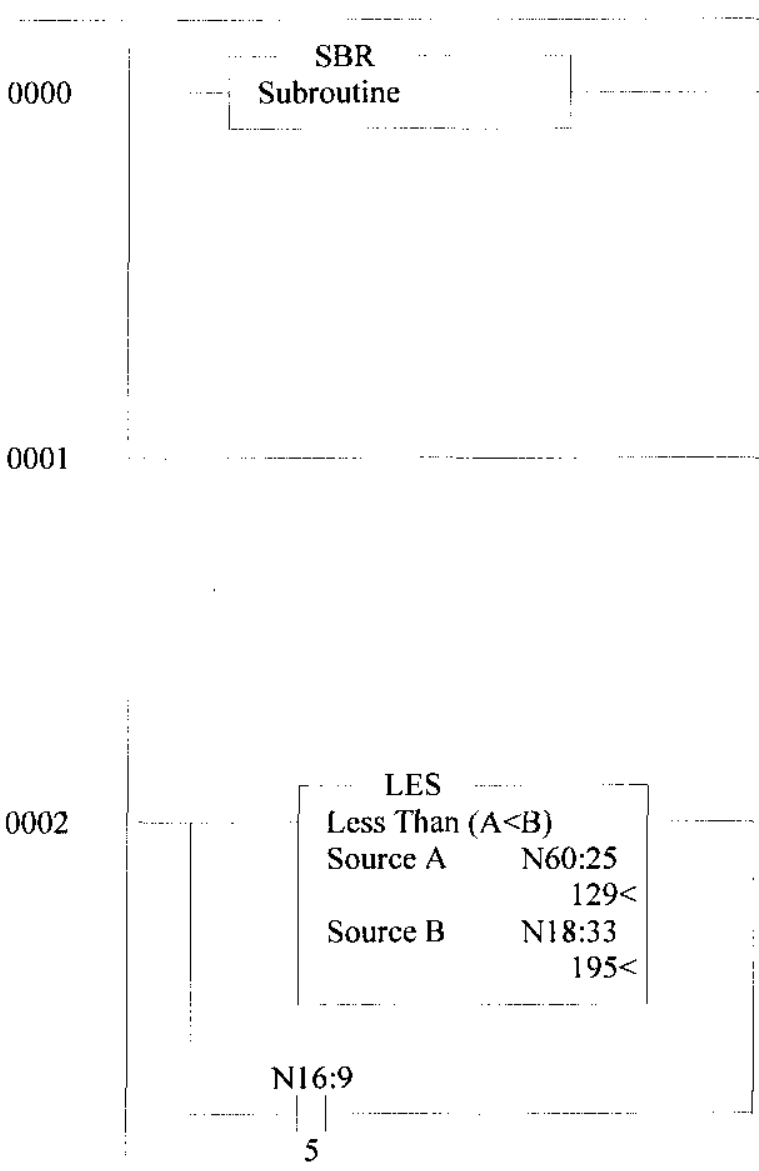

Total Rungs in File $=6$

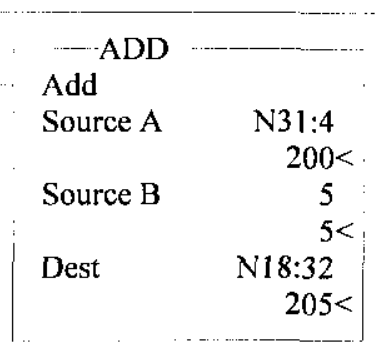

$\begin{array}{lc}-1 \text { SUB } & \\ \text { Subtract } & \\ \text { Source A } & \text { N31:4 } \\ \text { Source B } & 200< \\ & 5 \\ \text { Dest } & \text { N18:33 } \\ & 195<\end{array}$

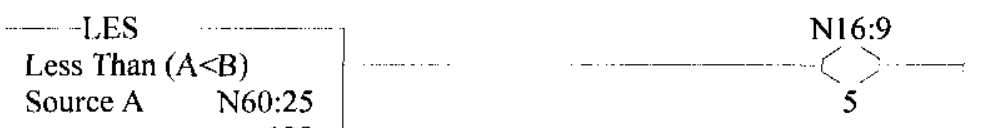

0003
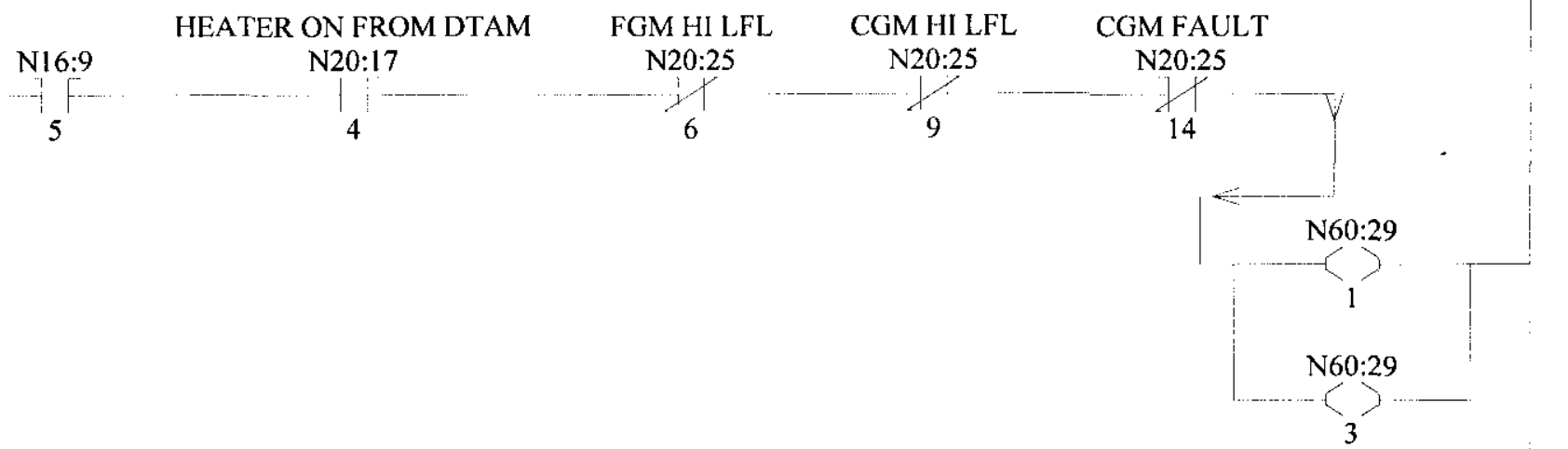

0004

$\begin{array}{cc} & 129< \\ \text { Source B } & \mathrm{N} 18: 32 \\ & 205<\end{array}$

$205<$ 
U109NEW1R1 PIC SKID N

LAD 10 - -- Total Rungs in File $=14$
RPP-5492

REVISION 0

APPENDIX B

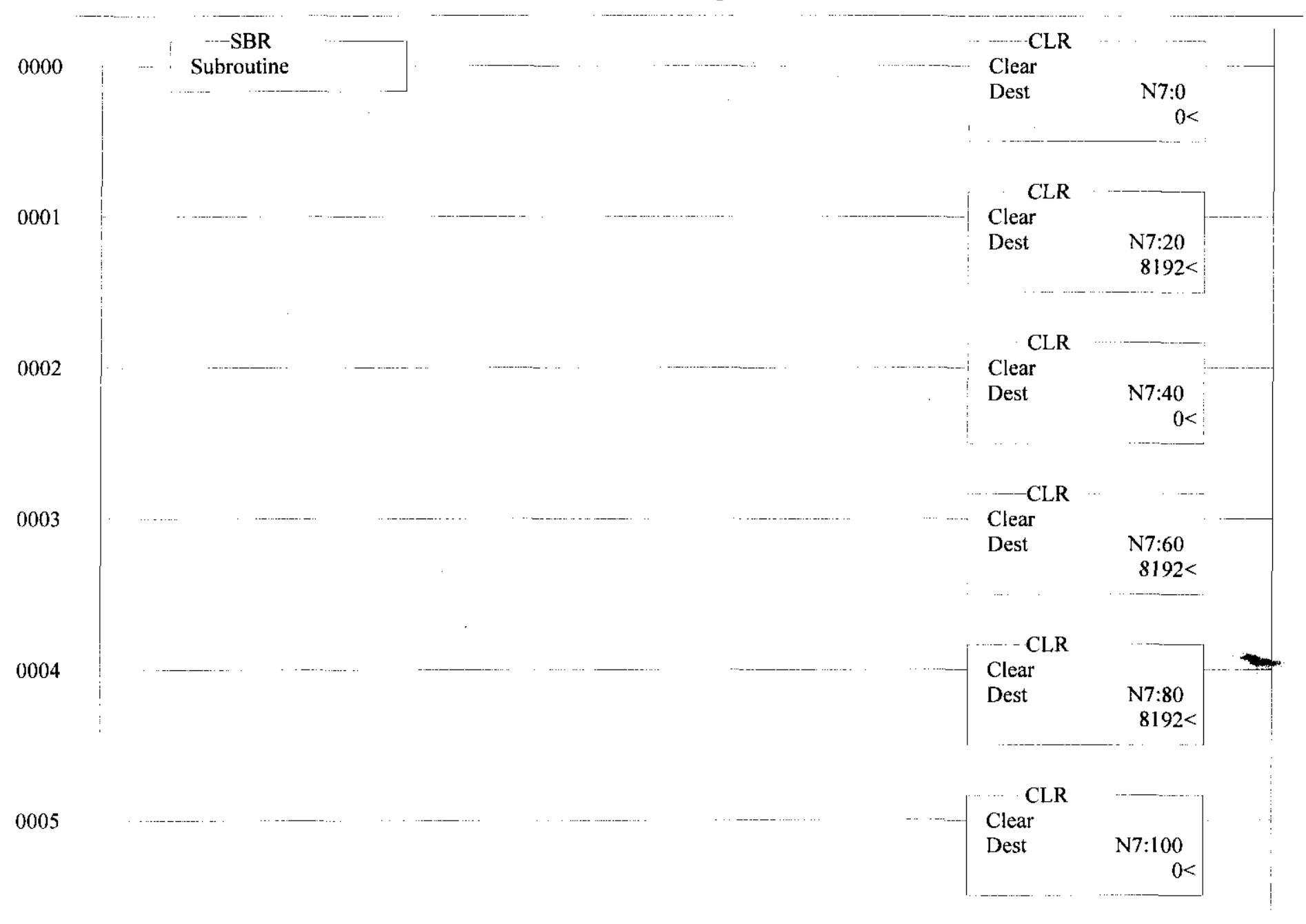

0006

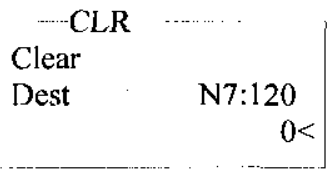

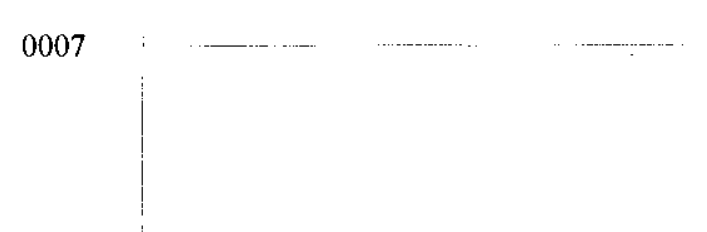

CLR

Clear

Dest
N7:140
$0<$

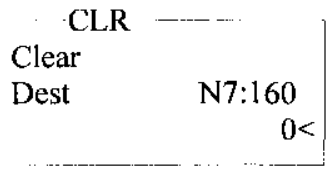

Clear

Dest

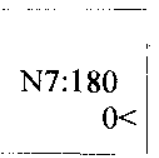


LAD 10 - - Total Rungs in File $=14$
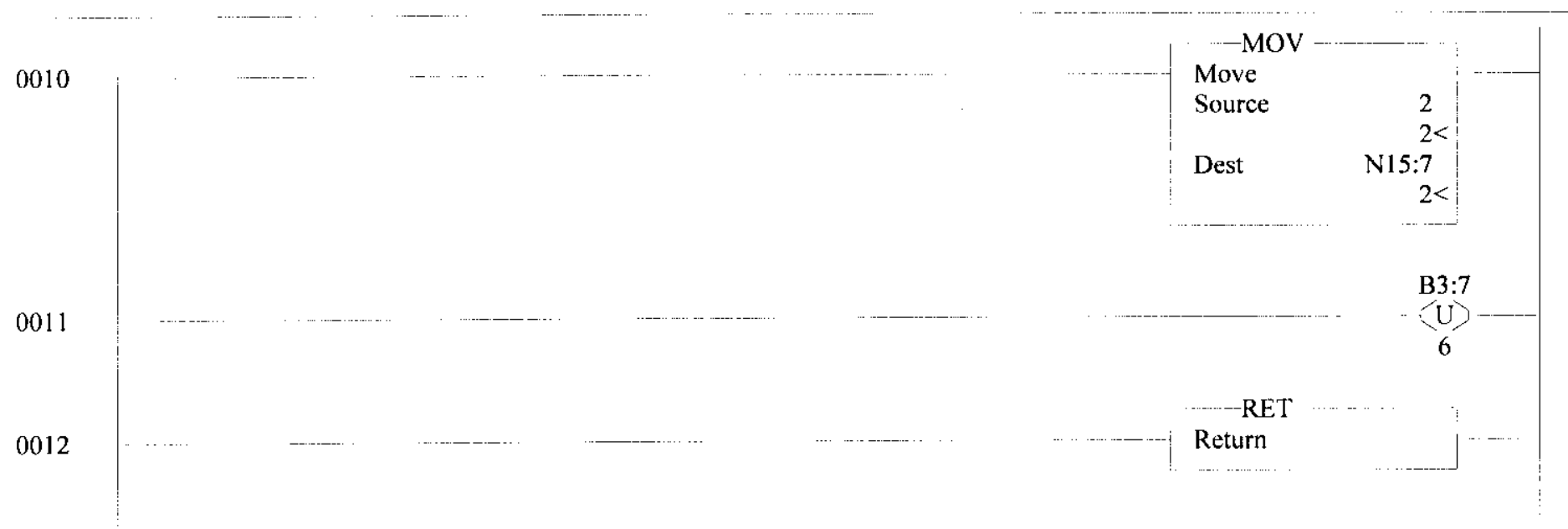

0013
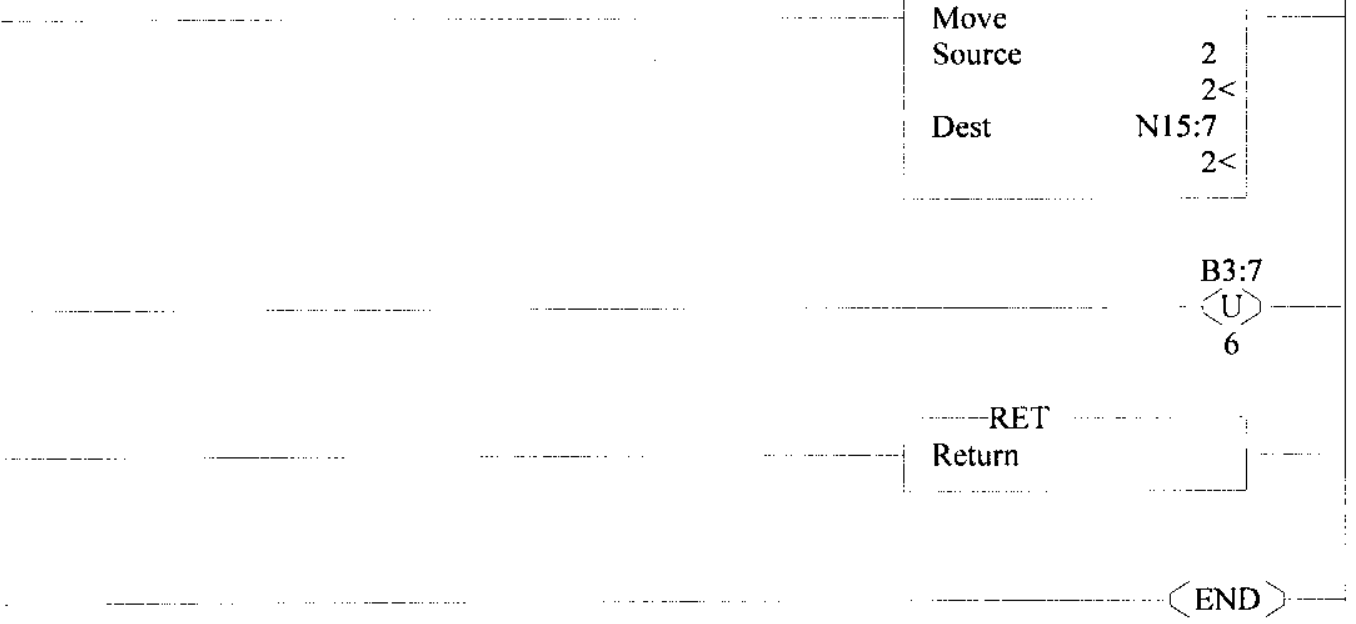

08:14:21 PAGE 2 Wednesday, March 15, 2000PAGE B 50 OF B 90 
LAD 11 - -- Total Rungs in File = 3

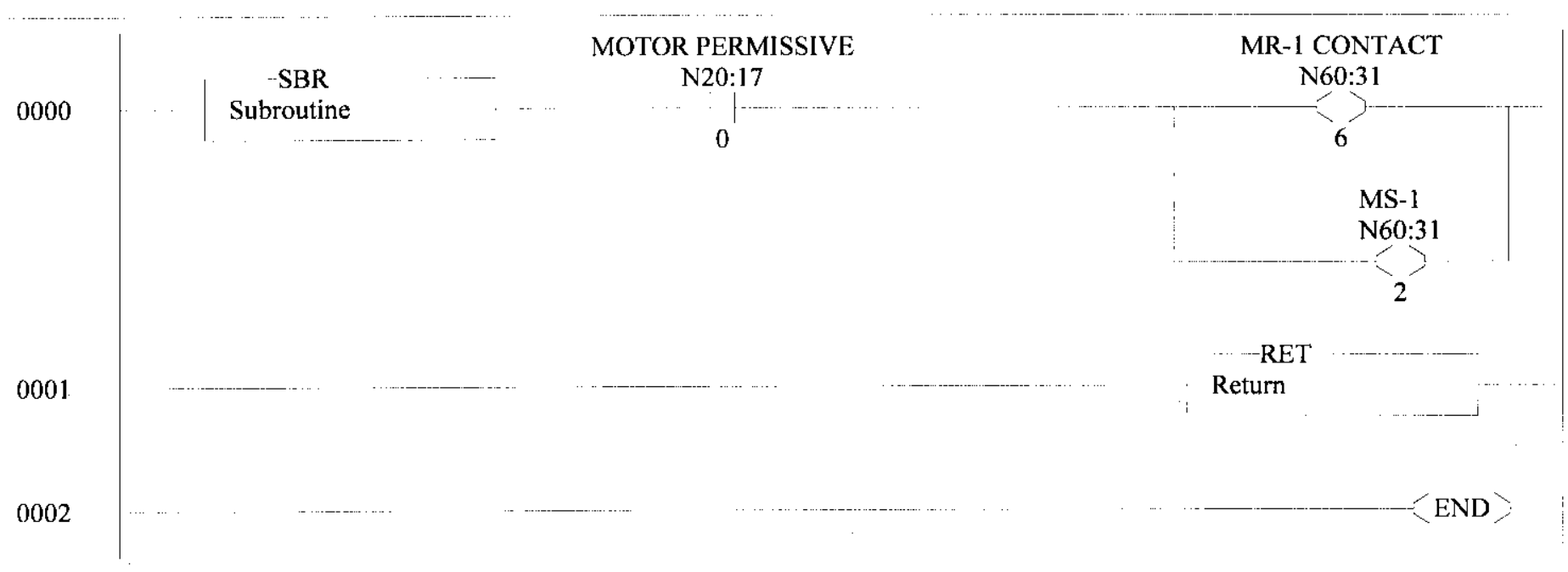




0000

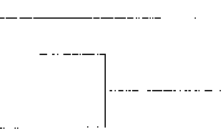

0001

$\left|\begin{array}{cc}\text { Not Equal } & \\ \text { Source A } & \text { N20:32 } \\ & 4< \\ \text { Source B } & \text { N40:15 } \\ & 4<\end{array}\right|$

-......JSR

Jump To Subroutine SBR File Number

$\mathrm{U}: 10$

\begin{tabular}{|lc} 
MOV & \\
Move & \\
Source & N20:32 \\
Dest & N40:16 \\
& $4<$
\end{tabular}

.......-.JSR

Jump To Subroutine

SBR File Number

$\begin{array}{lr}\quad \text { AND } & \\ \text { Bitwise AND } & \\ \text { Source A } & \text { S:9 } \\ & 3 \mathrm{~A} 35 \mathrm{~h}< \\ \text { Source B } & \mathrm{N} 15: 8 \\ & 0034 \mathrm{~h}< \\ \text { Dest } & \mathrm{N} 15: 9 \\ & 0034 \mathrm{~h}<\end{array}$

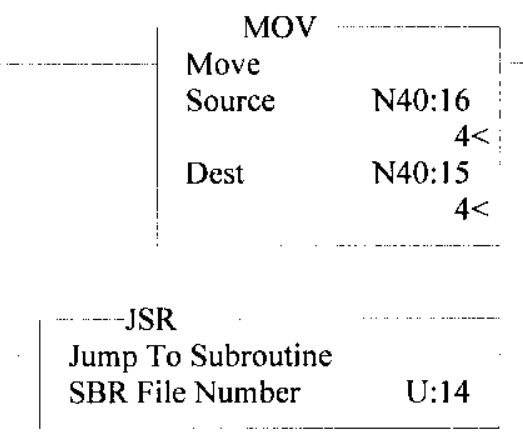

T4:32

RES

0002

NEQ

Not Equal

Source A

Source B
N15

$52<$

N15:11

$52<$
…....JSR

Jump To Subroutine SBR File Number

$\mathrm{U}: 10$

JSR

Jump To Subroutine SBR File Number

$\mathrm{U}: 14$

$\left|\begin{array}{lr}\text { Move } & \\ \text { Source } & \text { N15:9 } \\ & 52< \\ \text { Dest } & \text { N15:11 } \\ & 52<\end{array}\right|$

T4:32

RES 
LAD 12 - - Total Rungs in File $=10$

B3:7

0003 0004

0005

0006

0007
6
N20:17

$\mathrm{T} 4: 32$
JSR Jump To Subroutine SBR File Number
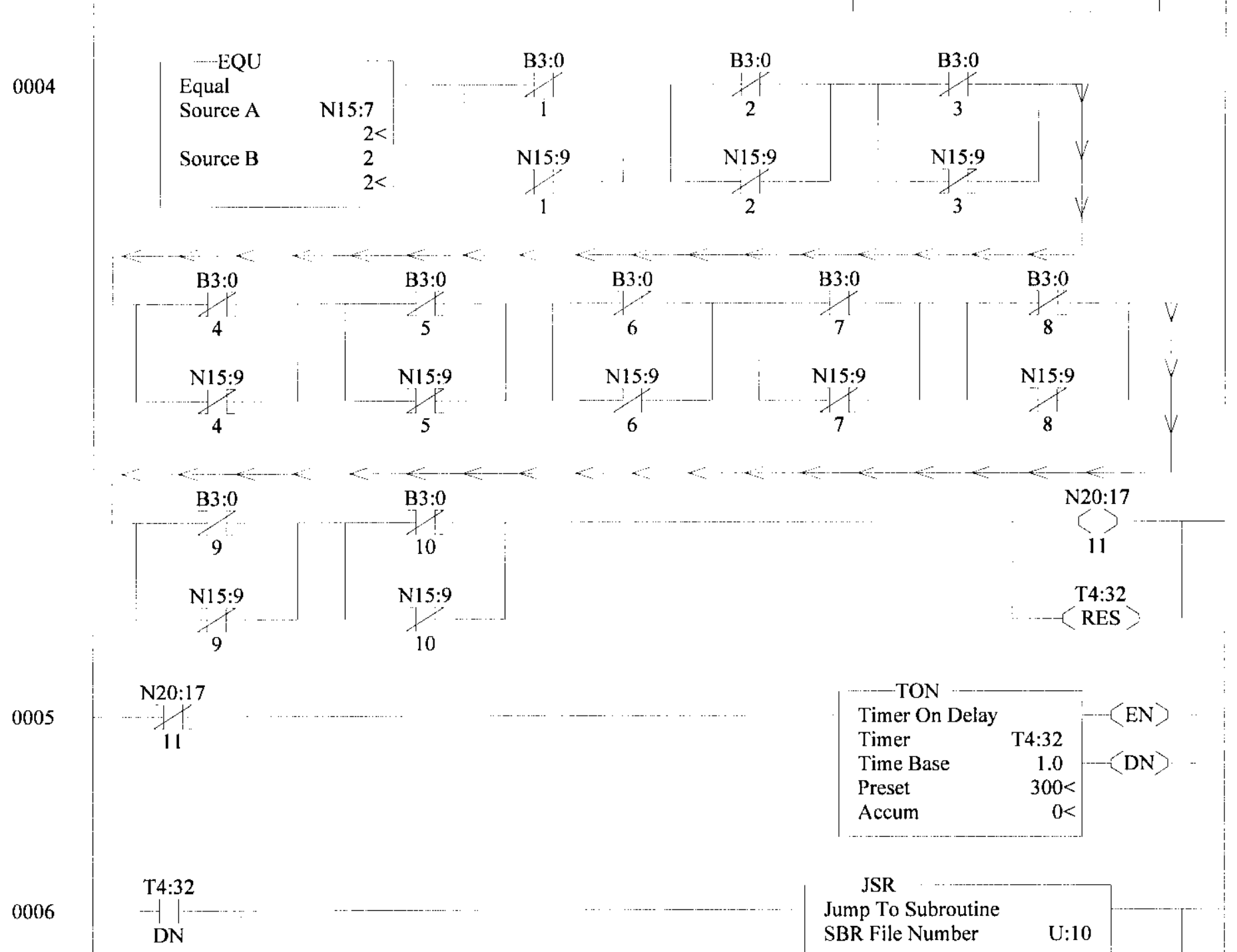

JSR Jump To Subroutine SBR File Number

U:10

SR

Jump To Subroutine SBR File Number U:14

T4:32 RES

NEQ Not Equal Source A Source B

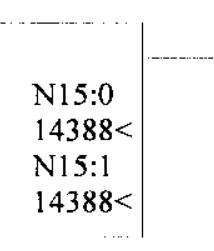

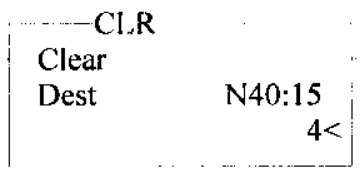




$$
\text { LAD } 12 \text { - -- Total Rungs in File }=10
$$

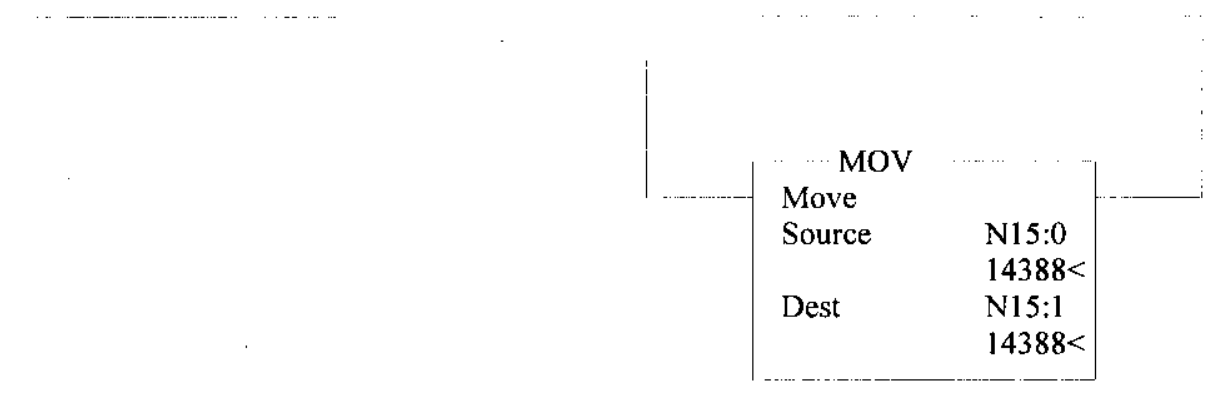


RPP-5492

U109NEW1RI PIC SKID N

REVISION 0

APPENDIX B

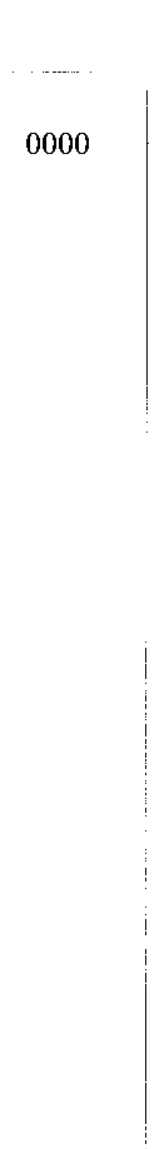

LAD 13 - -- Total Rungs in File $=6$
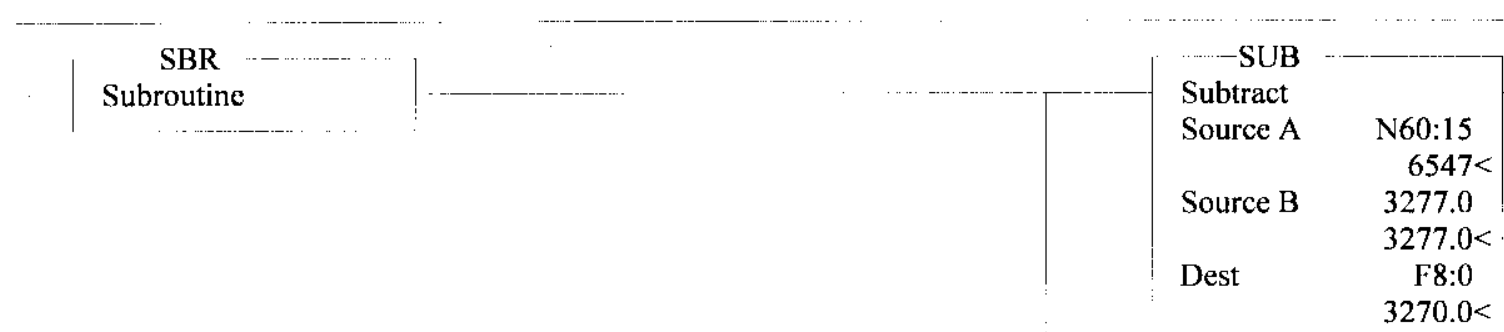

$$
\text { -.-DIV }
$$

Divide

Source A

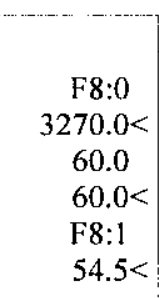

Source B

Dest $54.5<$

$\begin{array}{lc}\quad \text { ADD } & \\ \text { Add } & \mathrm{F} 8: 1 \\ \text { Source A } & 54.5< \\ & \mathrm{F} 8: 2 \\ \text { Source B } & \mathrm{F} 8: 2 \\ & 90.65894< \\ \text { Dest } & 90.65894<\end{array}$

GRT

0001

Greater Than $(\mathrm{A}>\mathrm{B})$

Source A

$\mathrm{F} 8: 2$

$\begin{array}{ll} & 90.65894< \\ \text { Source B } & 1638.37 \\ & 1638.37<\end{array}$

CTU

Count Up

Counter

Preset

Accum

$\begin{array}{lc}\text { SUB } & \\ \text { Subtract } & \\ \text { Source A } & \text { F8:2 } \\ & 90.65894< \\ \text { Source B } & 1638.37 \\ & 1638.37< \\ \text { Dest } & \text { F8:2 } \\ & 90.65894<\end{array}$

C5:1

CU

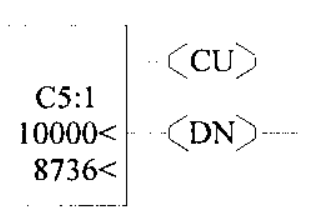

C5:1

0002

DN

C5:2

CU 
LAD 13 - --- Total Rungs in File $=6$
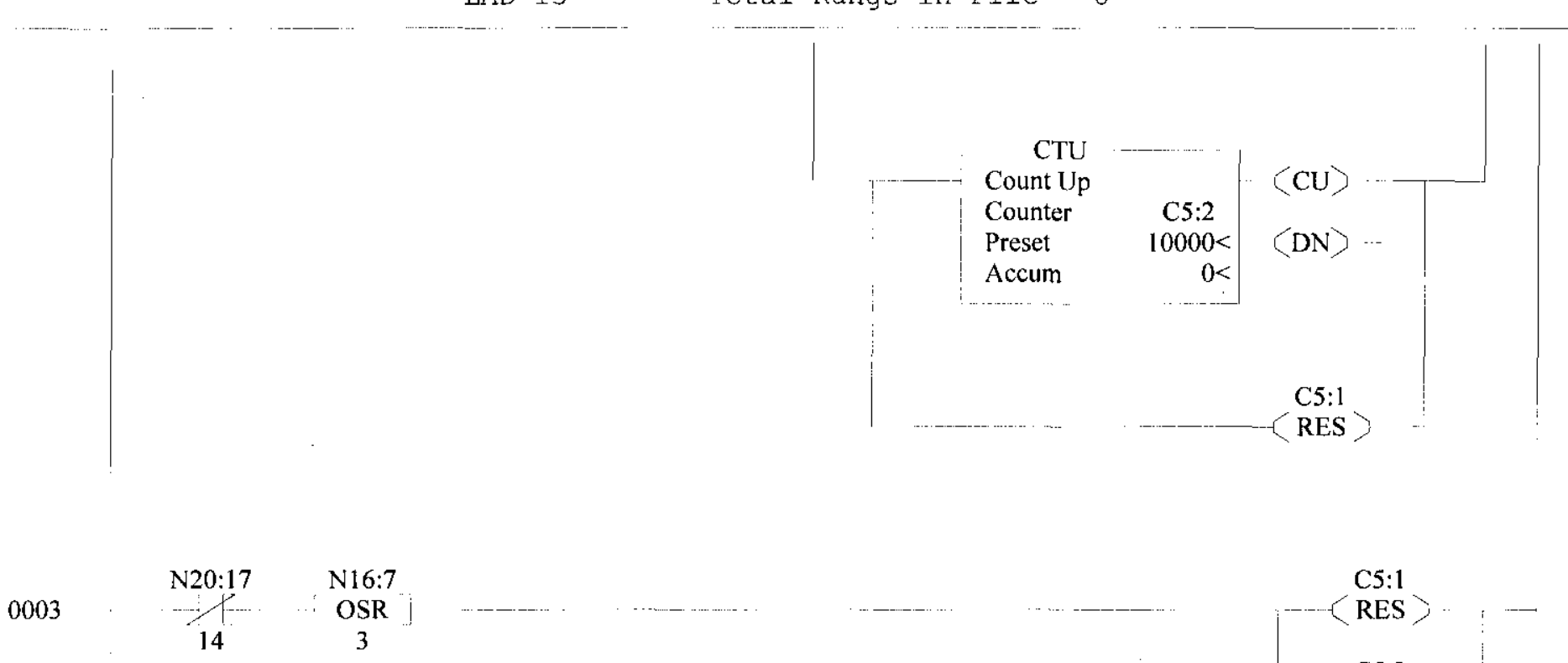

0004
0005

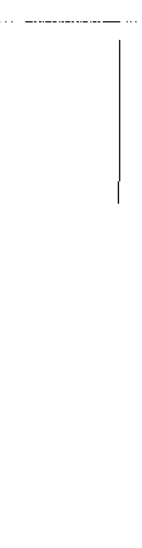

$$
14 \quad 3
$$
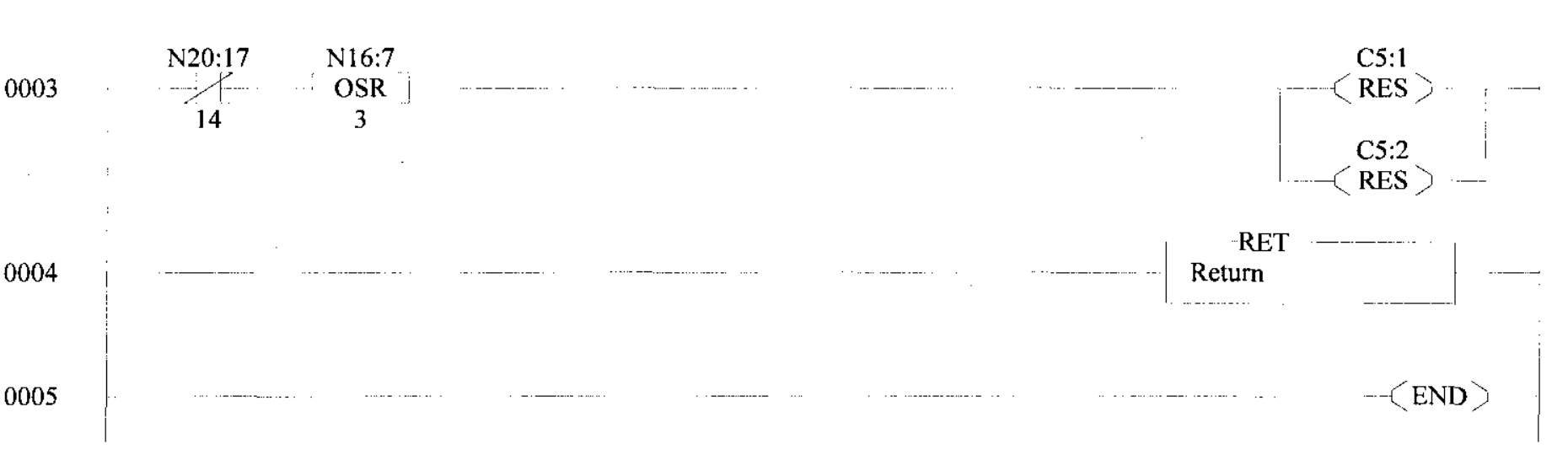

08:14:21 PAGE 2 Wednesday, March 15, 2000PAGE B 5 6 OF B 90 
RPP-5492

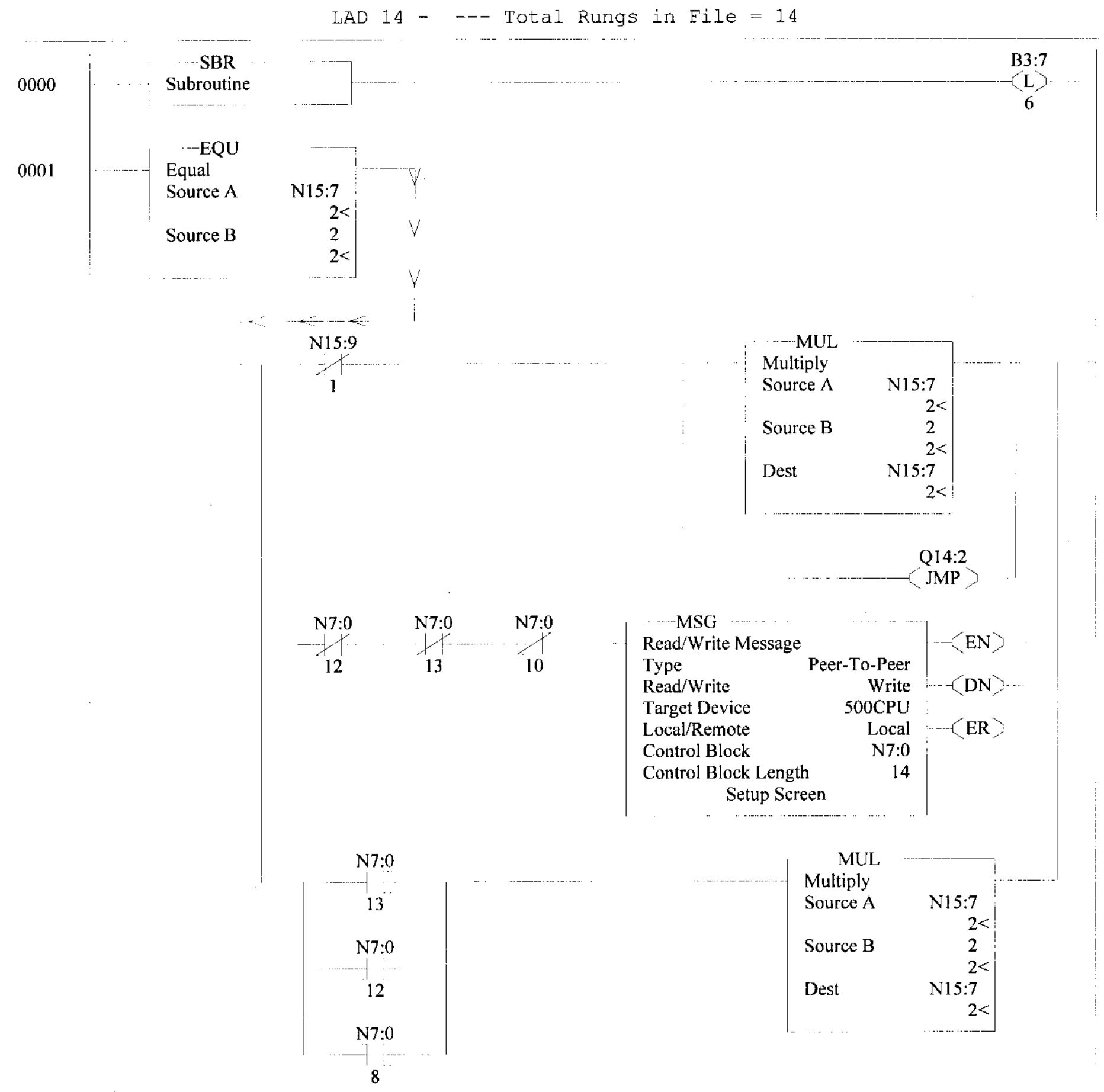

Multiply

Source A

Source B

Dest

6
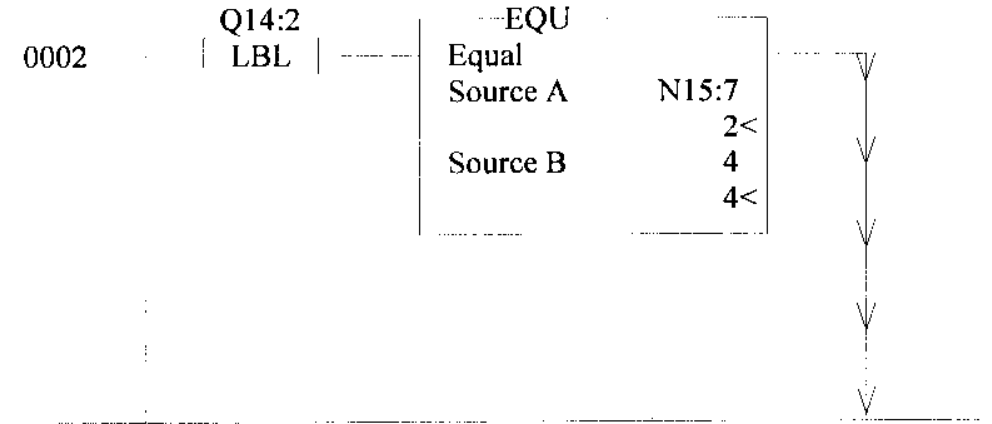
LAD 14 - -- Total Rungs in File $=14$
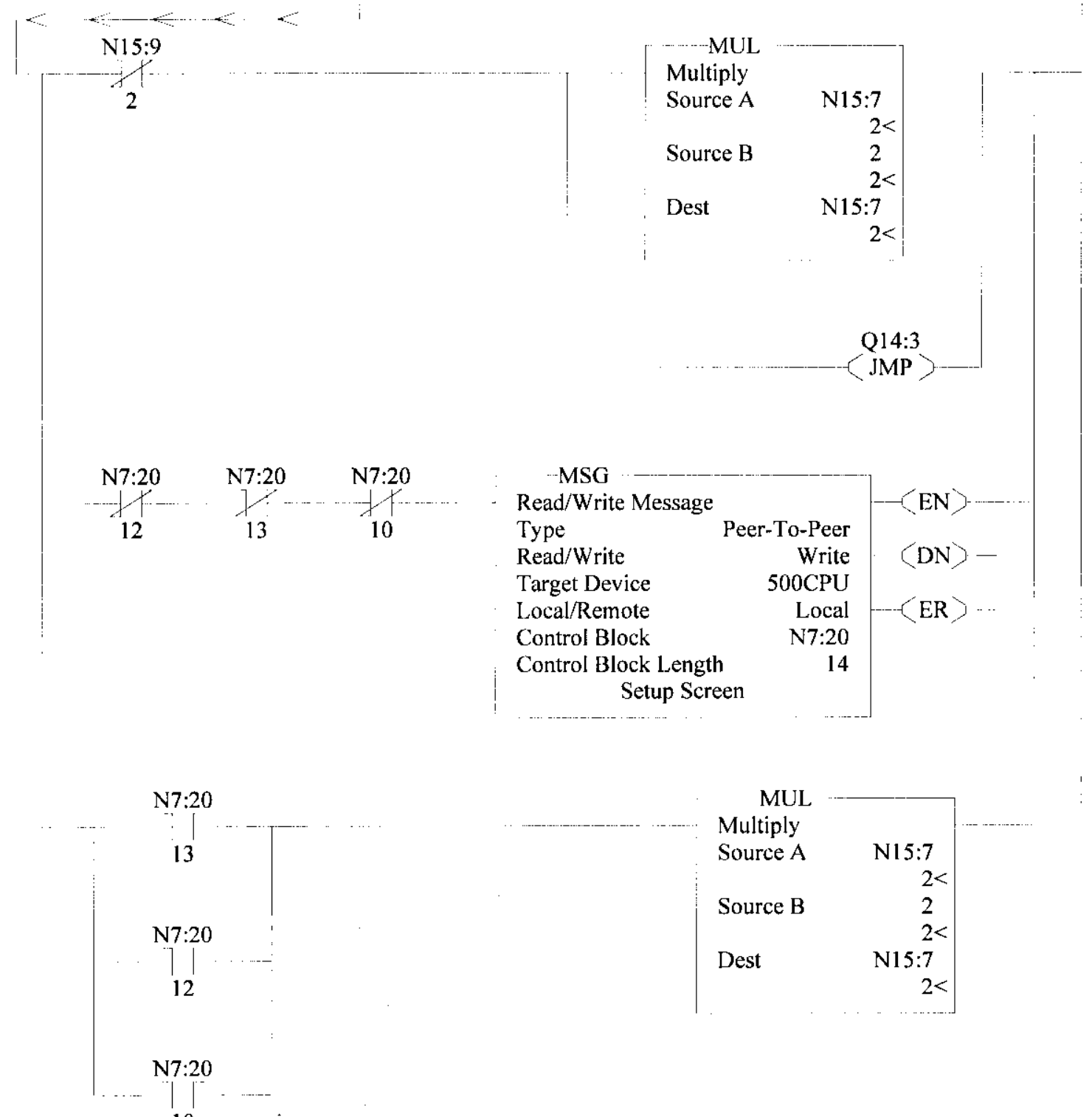

MUL

Multiply

Source A

Source B

Dest

\begin{tabular}{c}
\hline N15:7 \\
$2<$ \\
2 \\
$2<$ \\
N15:7 \\
$2<$
\end{tabular}

10

Q14:3

LBL |

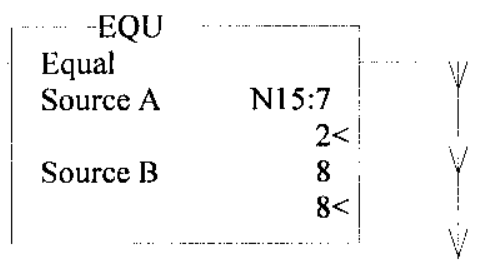

PAge B 58 of B 90 
LAD 14 - -- Total Rungs in File $=14$

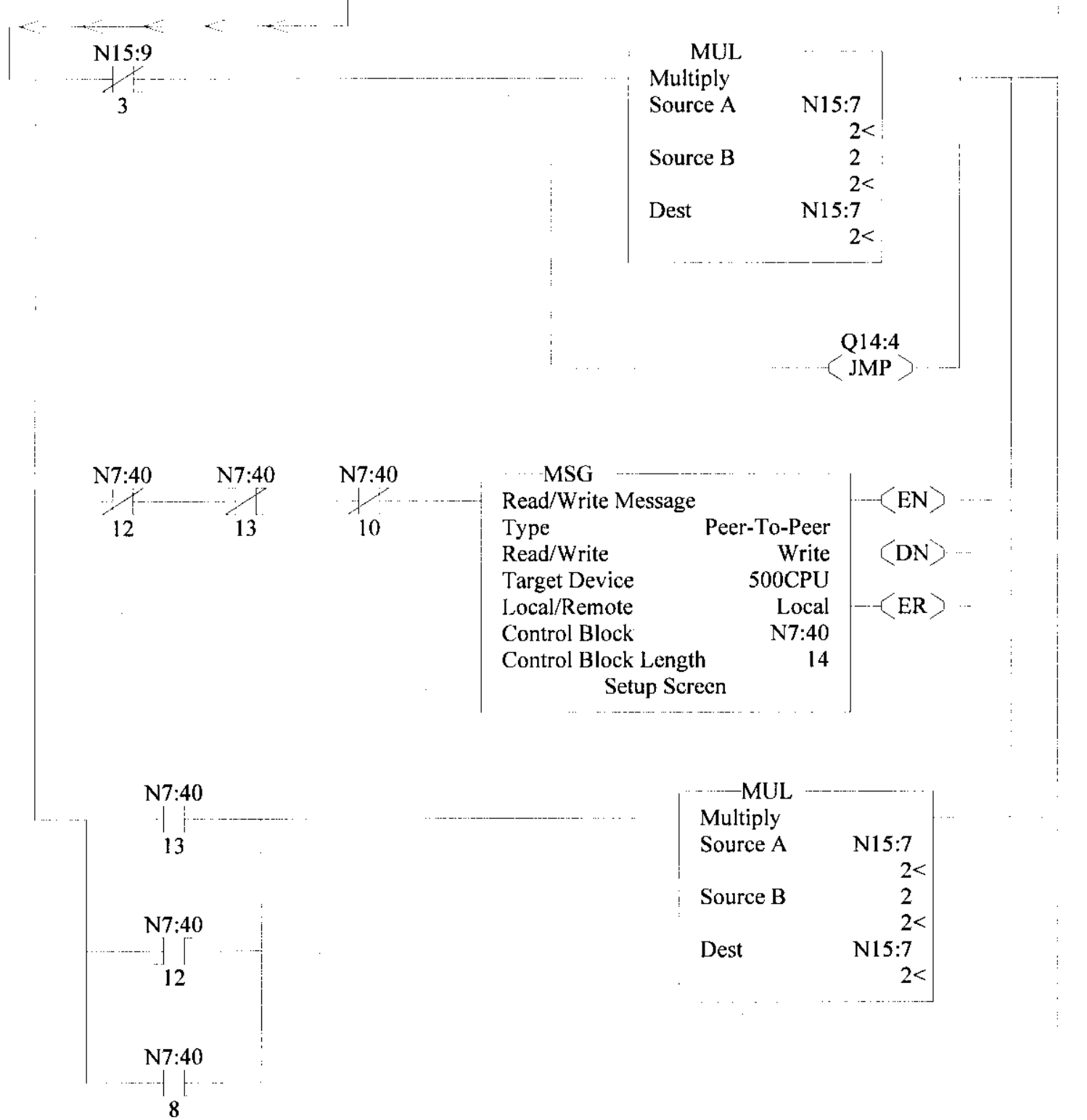

0004

Q14:4

LBL _ I

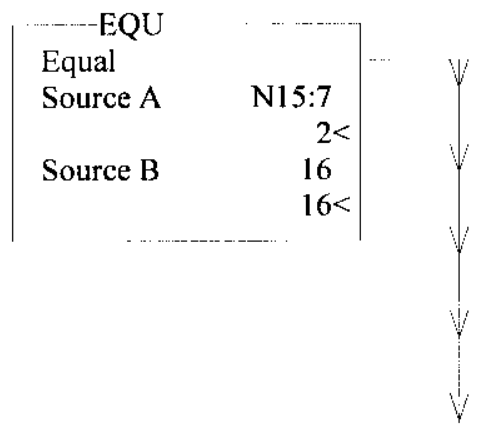


LAD 14 - - Total Rungs in File $=14$

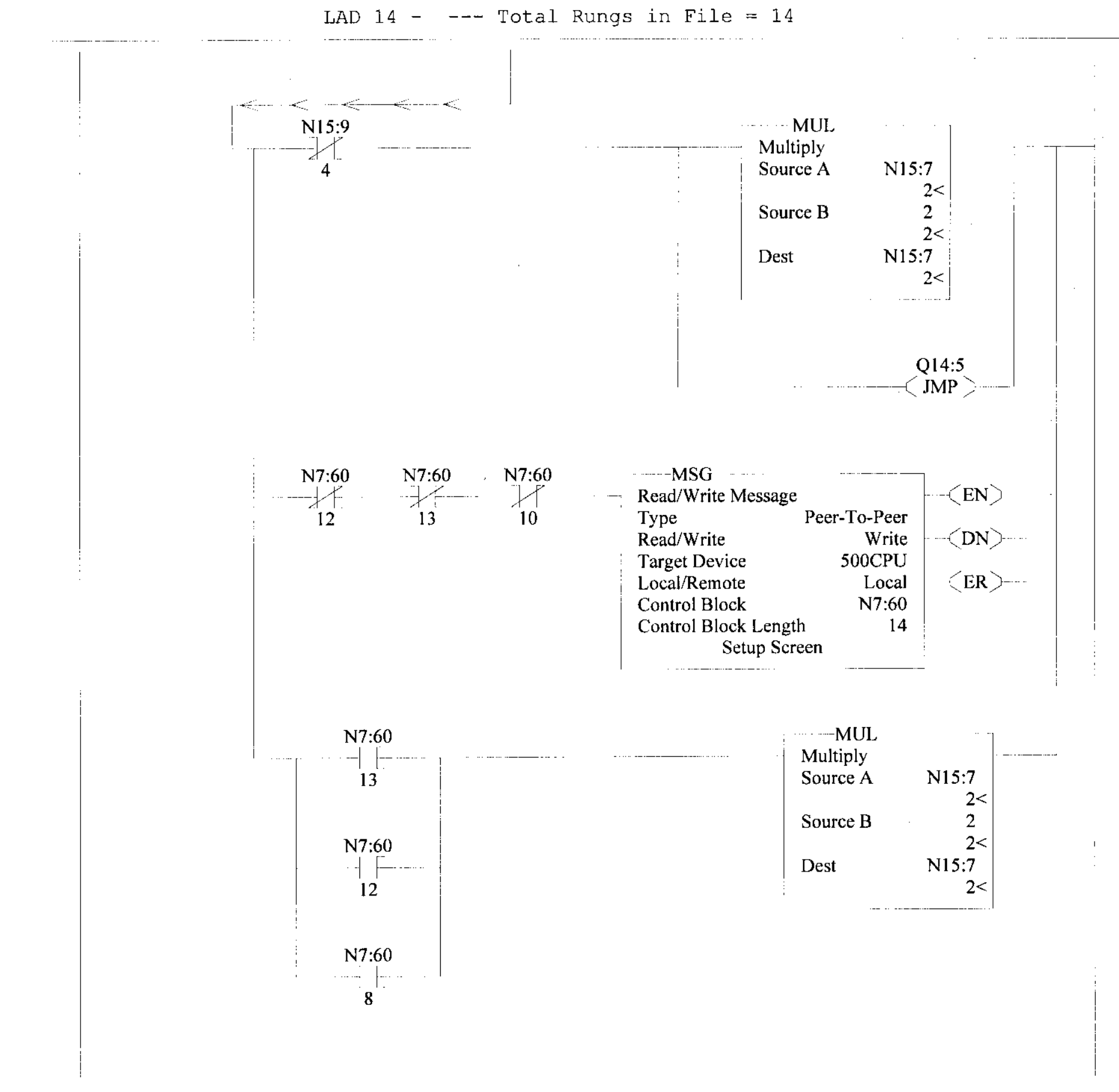

0005

Q14:5

$-\mathrm{EQU}$

Equal

Source A N15:7

Source B

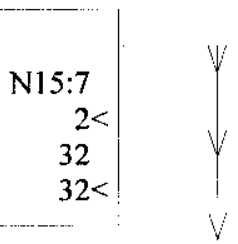

Multiply

Source A

Source B

Dest

$\begin{array}{cc}\text { LBL } & \text { Equal } \\ \text { Source A } & \text { N15:7 } \\ & 2< \\ \text { Source B } & 32 \\ & 32<\end{array}$

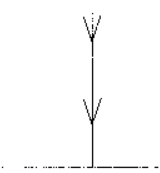




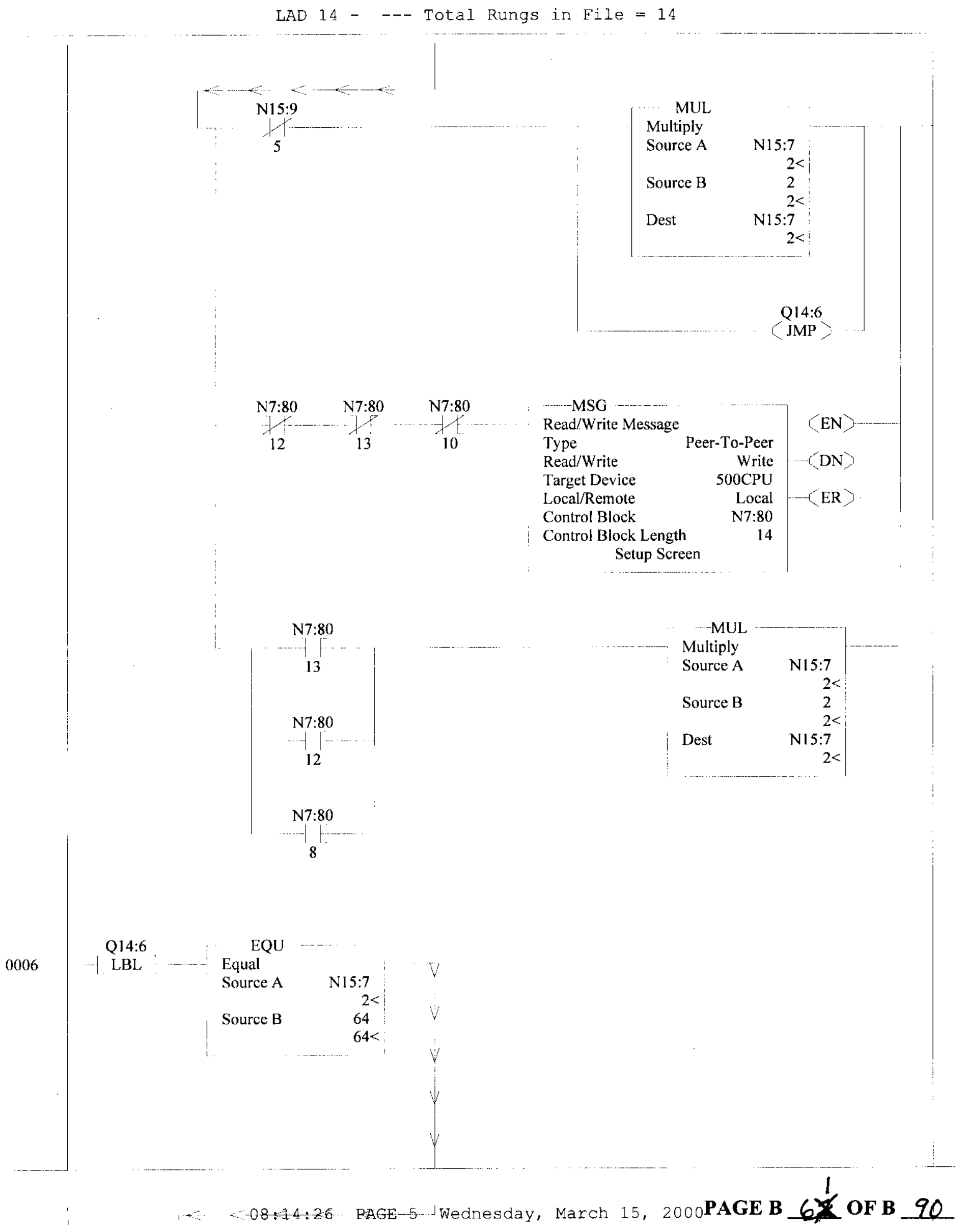




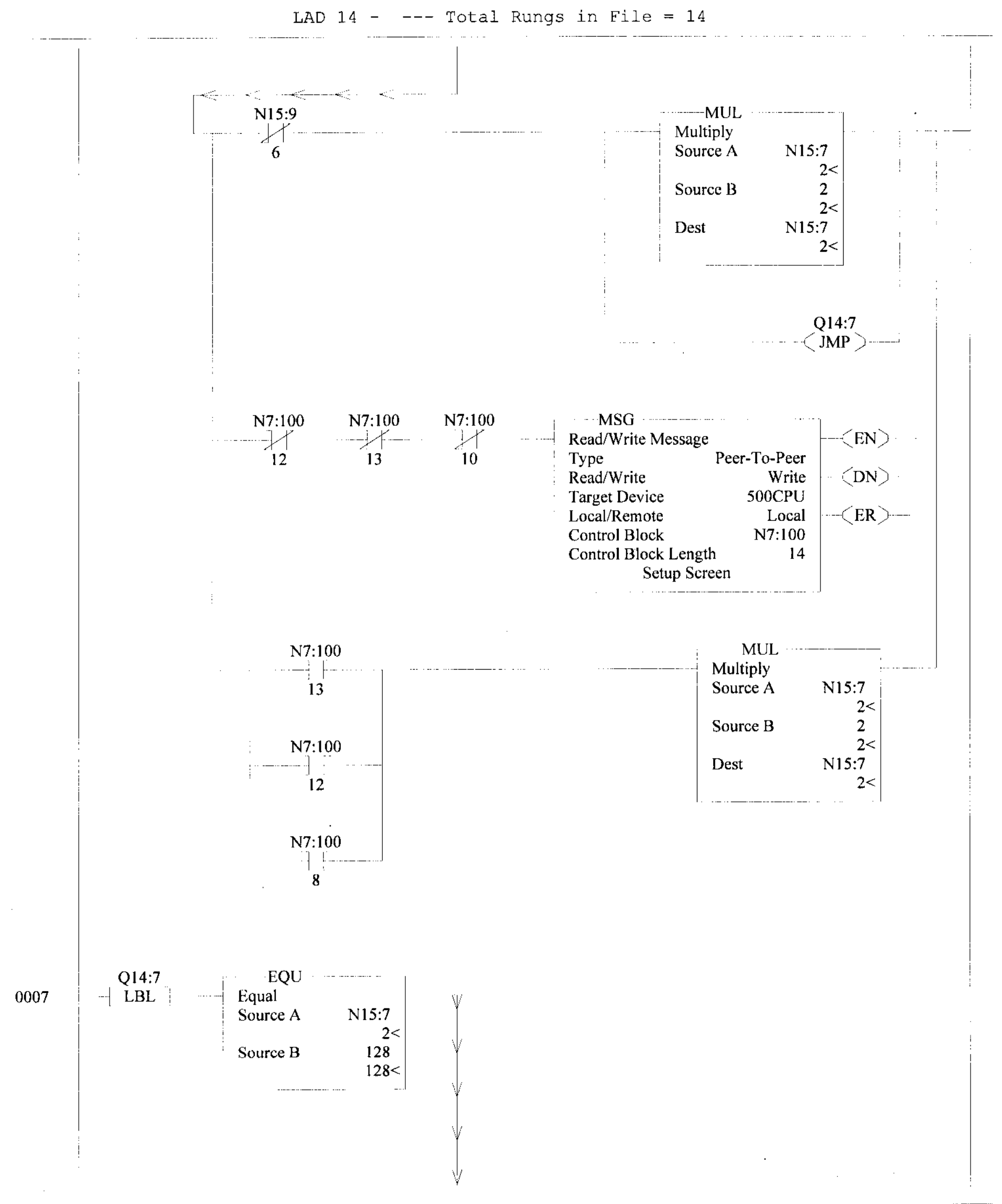


LAD 14 - -- Total Rungs in File = 14
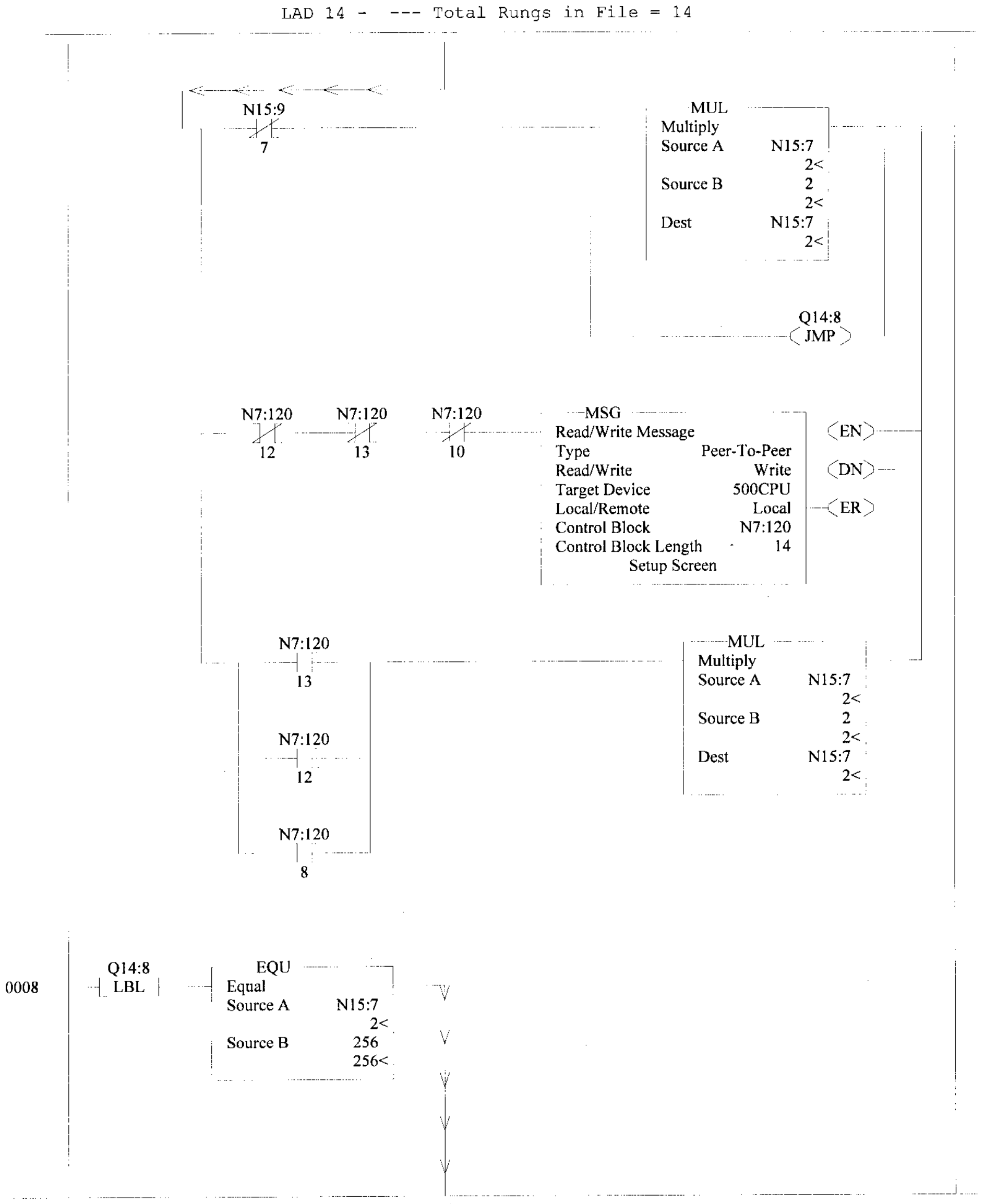
LAD 14 - -- Total Rungs in File $=14$

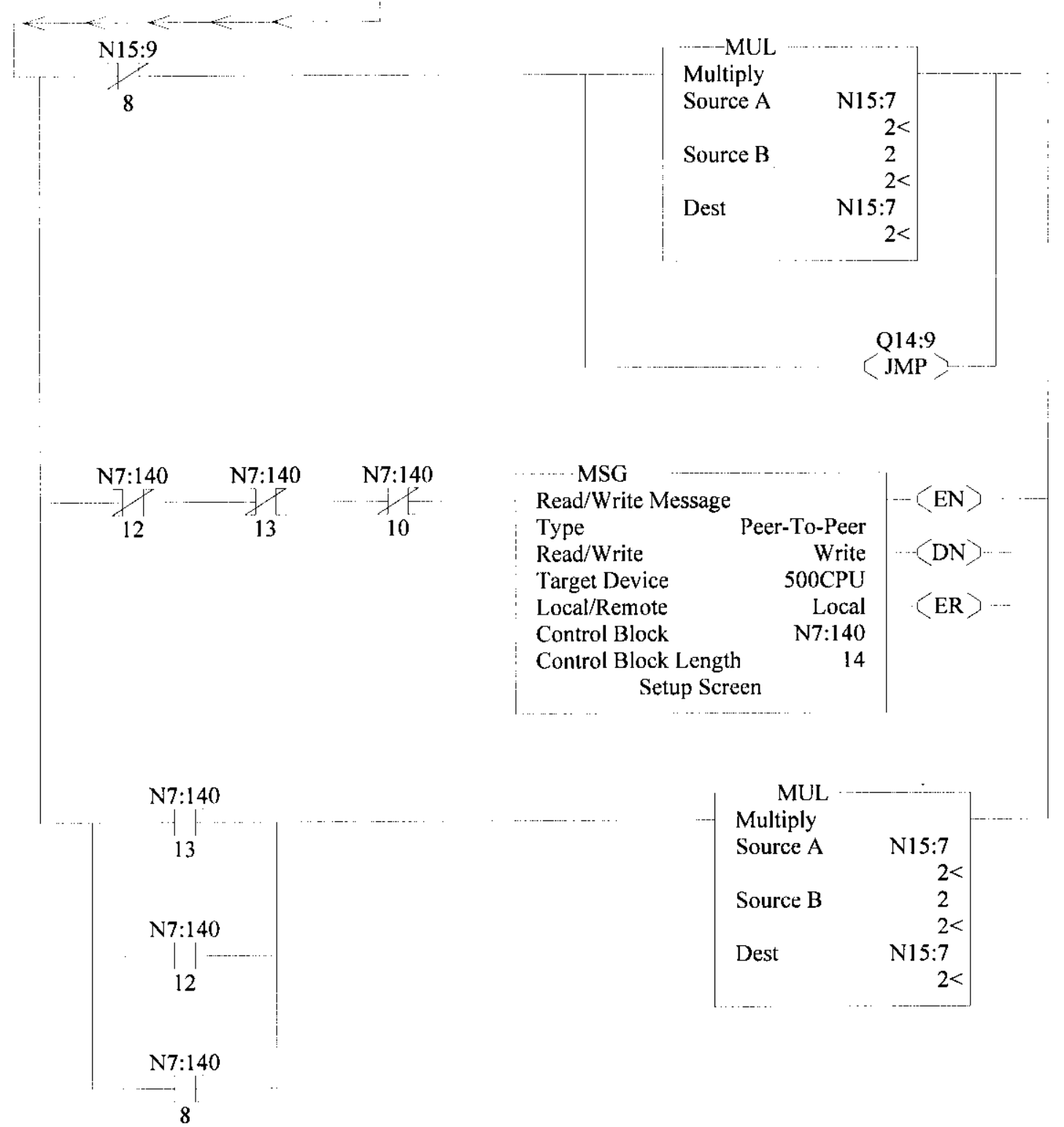

Q14:9

LBL

$\begin{array}{lc}\quad \text { EQU } & \\ \text { Equal } & \text { N15:7 } \\ \text { Source A } & 2< \\ & 512 \\ \text { Source B } & 512< \\ & \end{array}$

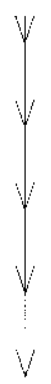


LAD 14 - $\ldots$ Total Rungs in File $=14$

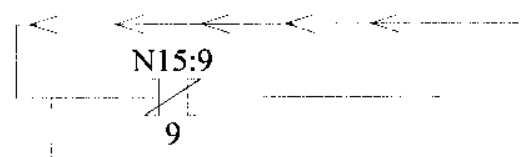

$\begin{array}{lc}\text { Multiply } & \\ \text { Source A } & \text { N15:7 } \\ & 2< \\ \text { Source B } & 2 \\ & 2< \\ \text { Dest } & \text { N15:7 } \\ & 2<\end{array}$
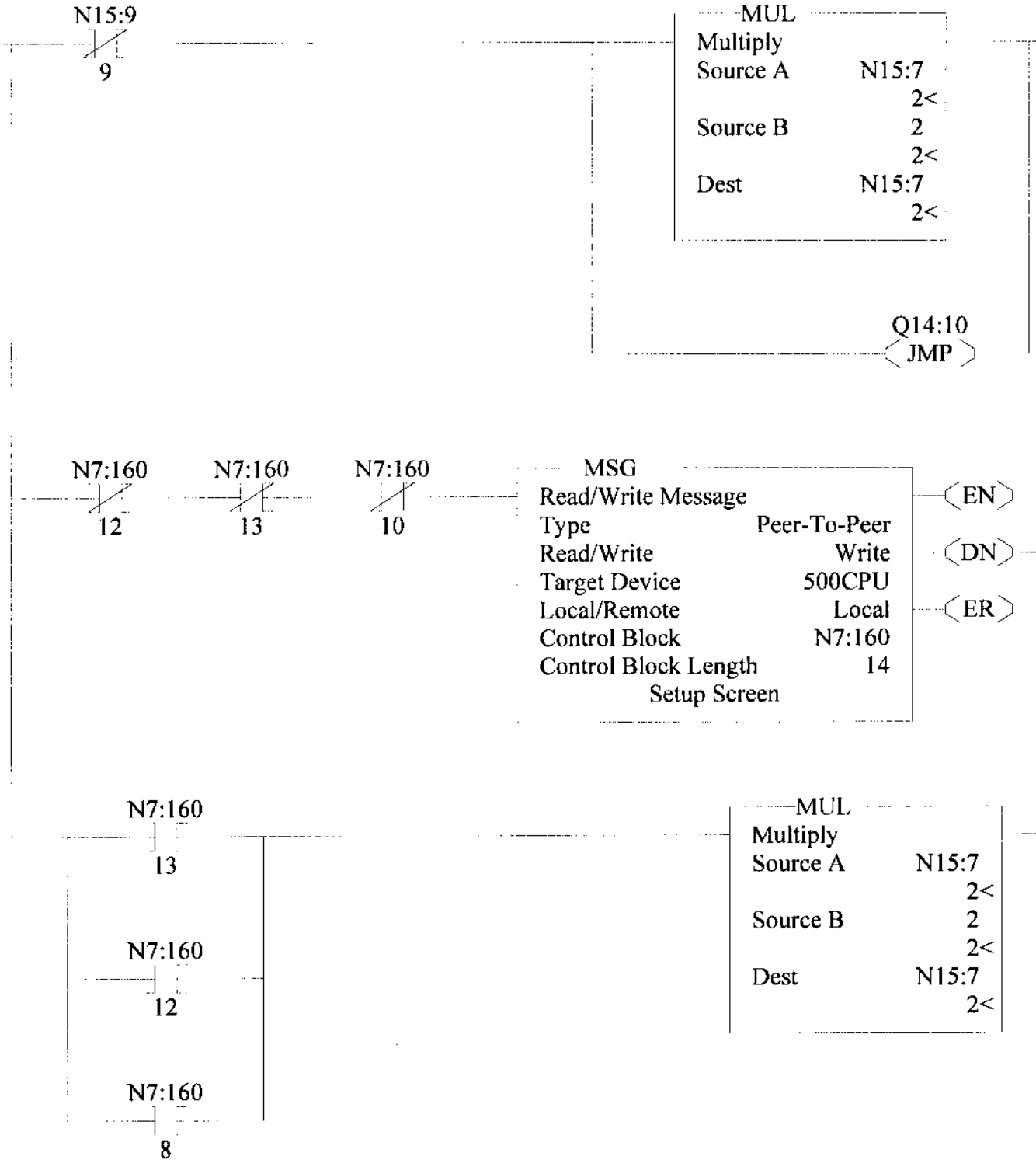

\begin{tabular}{lc|}
$\begin{array}{l}\text { Multiply } \\
\text { Mult }\end{array}$ & \\
Source A & N15:7 \\
Source B & $2<$ \\
& 2 \\
Dest & N15:7 \\
& $2<$
\end{tabular}

0010

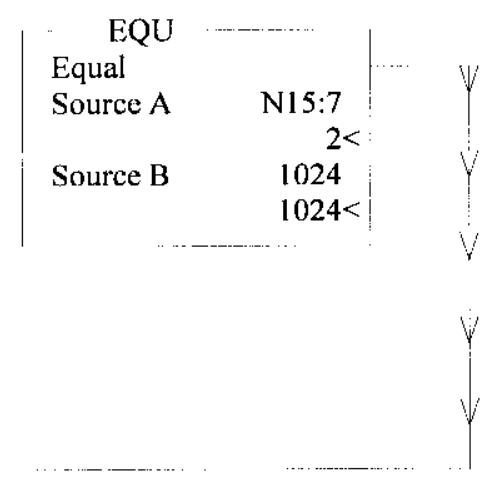

Q14:10

LBL

$1<08 \times 14: 26$ PAGE 9 Wednesday, March 15,2000 PAGE B 66 OF B 90 
LAD 14 - -- Total Rungs in File $=14$

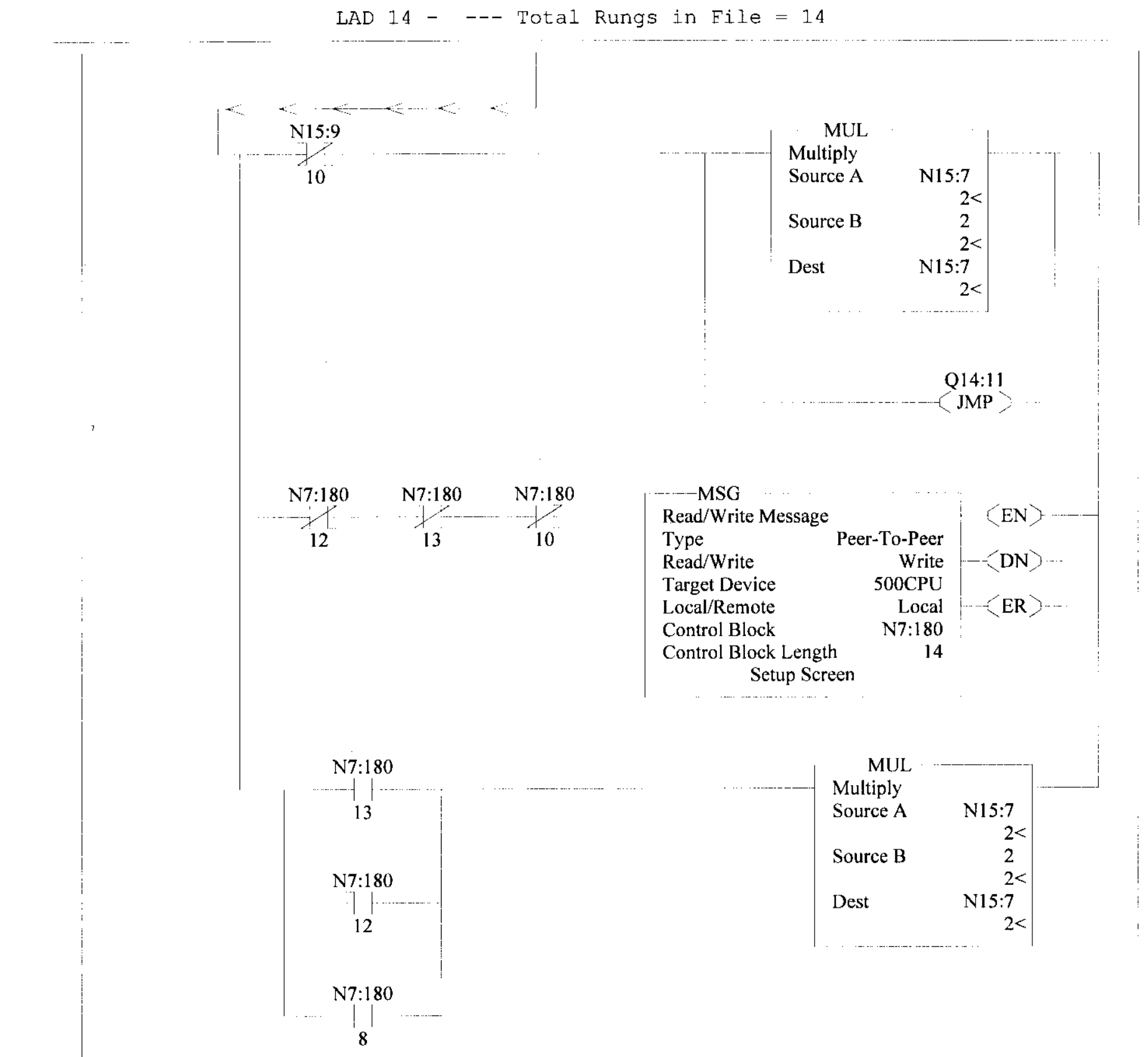

Q14:11

LBL

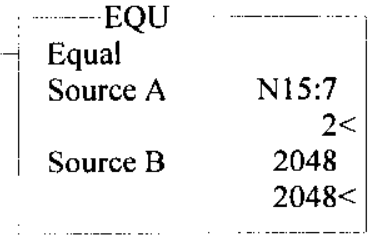

Read/Write

Read/Write

Target Device

ntrol Block

Setup Screen

Source A

Source B

Dest

5:7
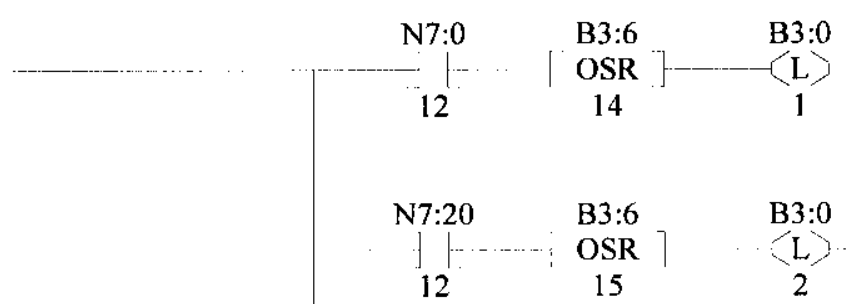

08:14:26 PAGE 10 Wednesday, March 15, 200 PAGE B 6 Z OF B 90 


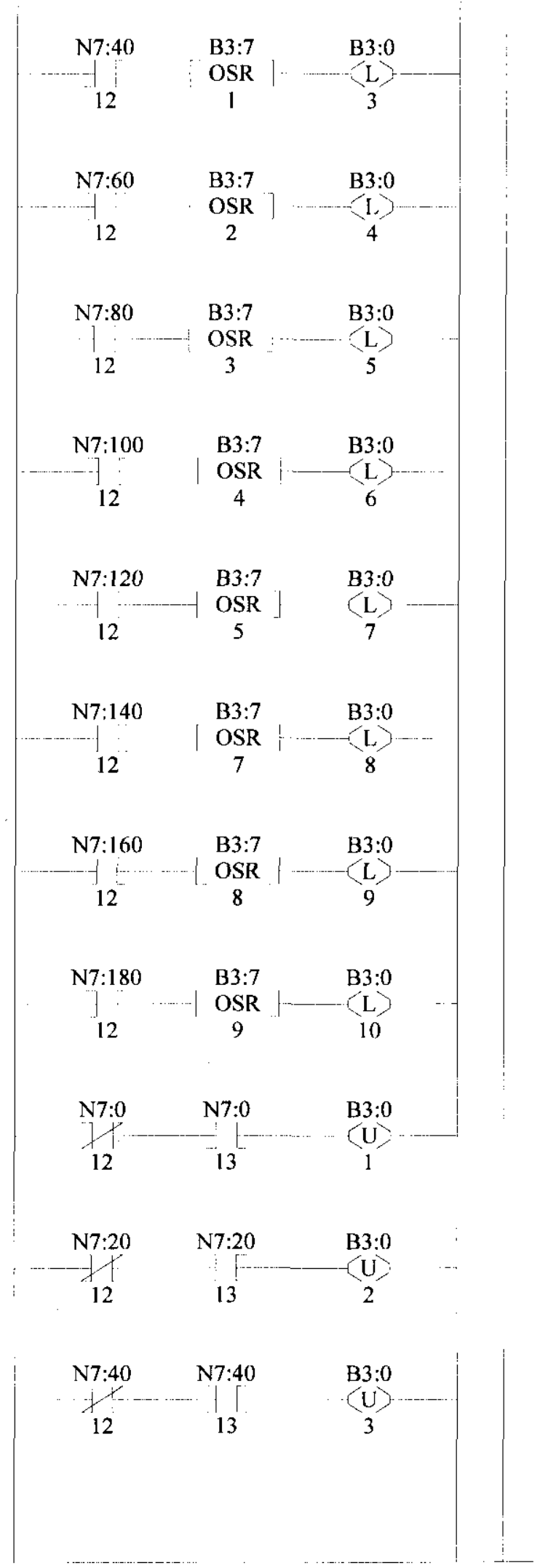


LAD 14 - -- Total Rungs in File $=14$

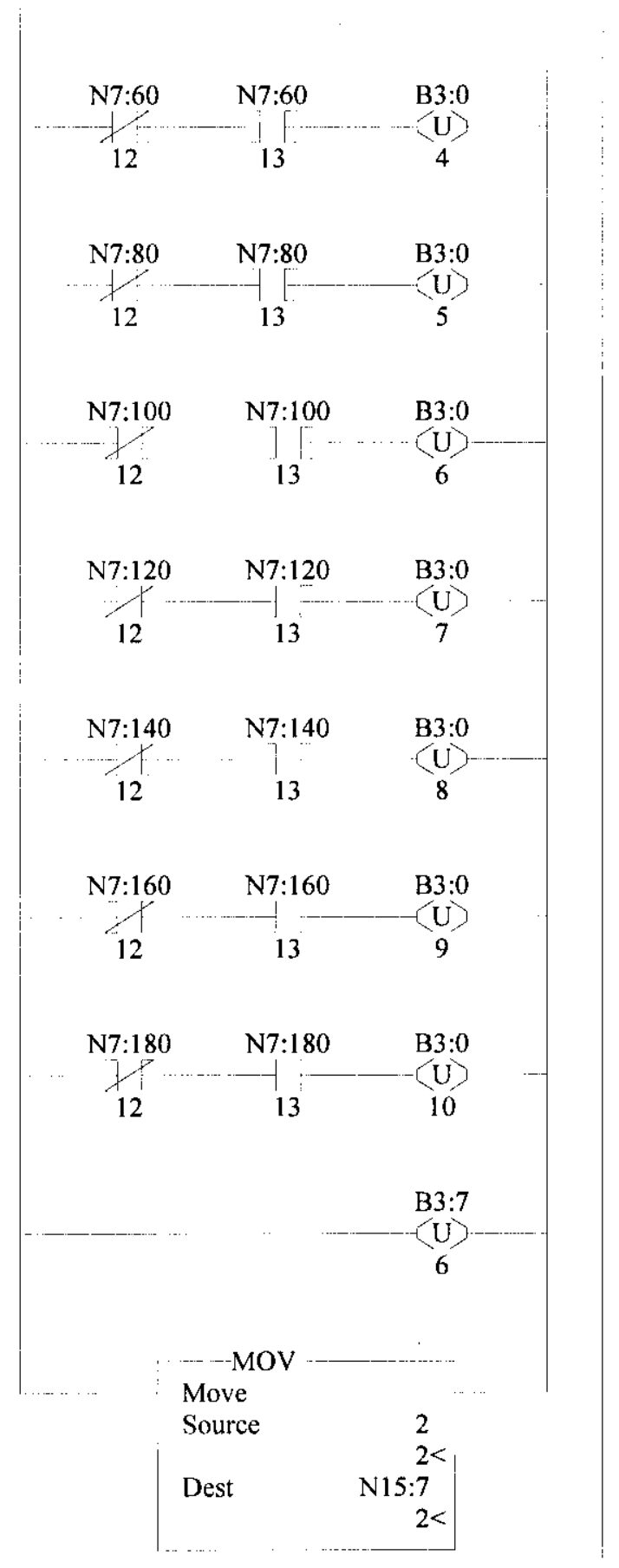

0012

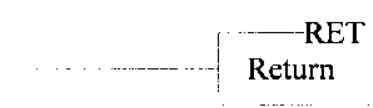

0013 


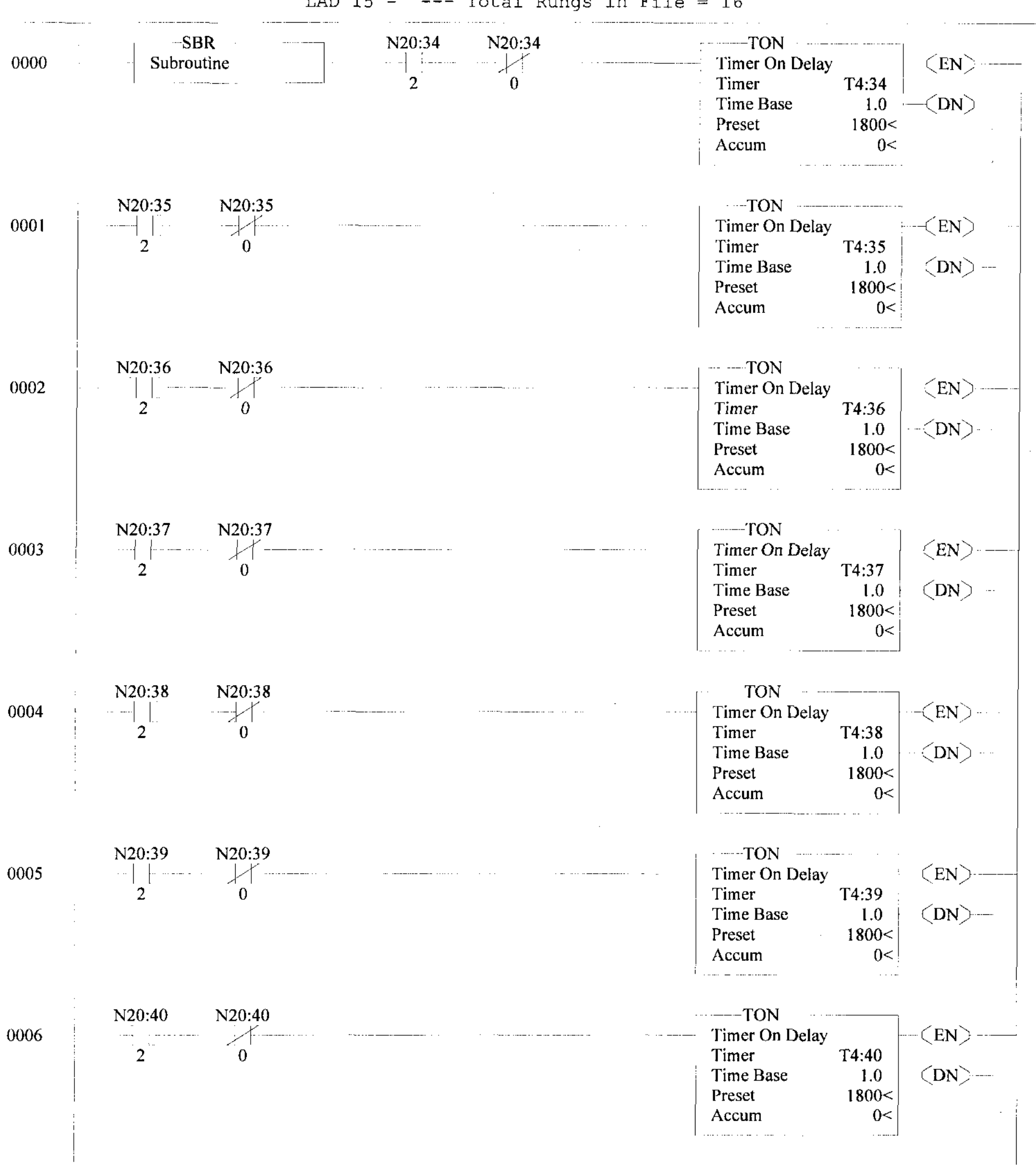


LAD 15 - -- Total Rungs in File $=16$

\begin{tabular}{c|cc} 
& $\mathrm{N} 20: 41$ & $\mathrm{~N} 20: 41$ \\
0007 & 2 & 0 \\
0008 & & \\
& & \\
& $\mathrm{~N} 20: 42$ & $\mathrm{~N} 20: 42$ \\
2 & 0
\end{tabular}

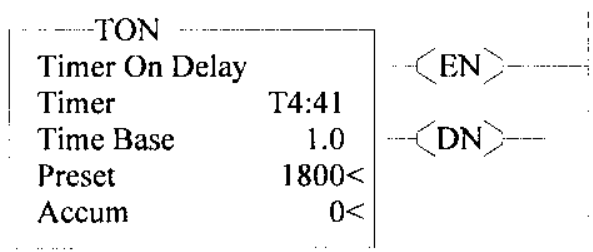

0009

N20:43 N20:43

2

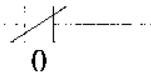

$$
\text { -...- TON }
$$

Timer On Delay

Timer

Time Base

Preset

Accum

A.........

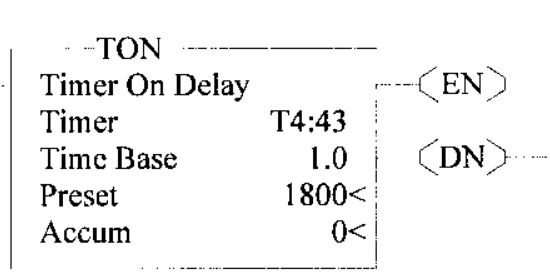

$0010 \quad \stackrel{\mathrm{N} 20: 44}{2} \quad{ }_{0}^{\mathrm{N} 20: 44}$

0011
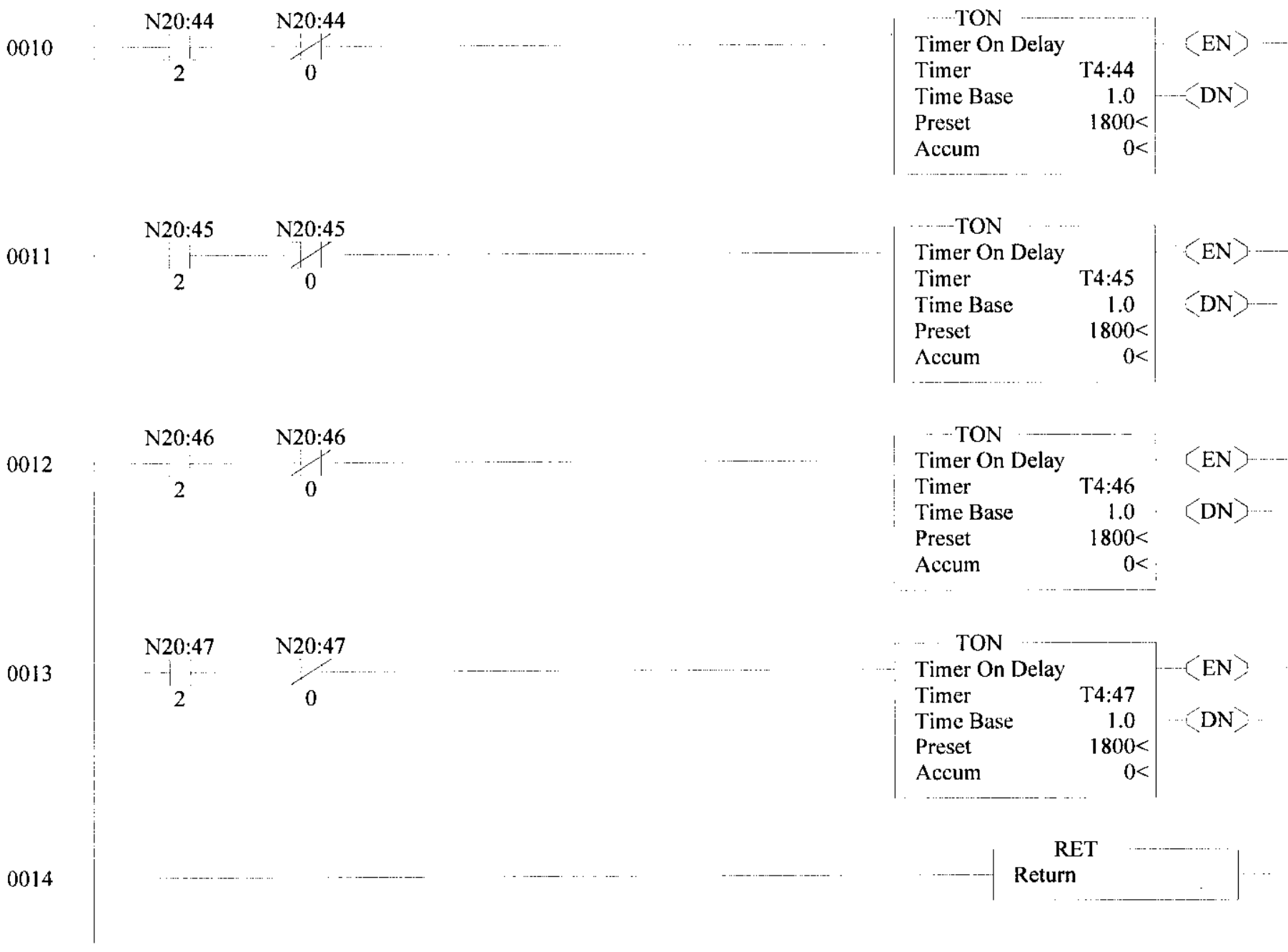

Accum

$$
\text { -.. TON }
$$

Timer On Delay

Timer

Time Base

Preset

Accum
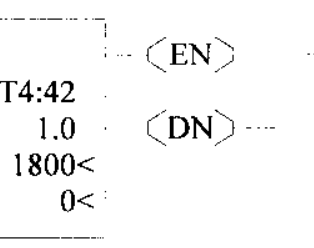


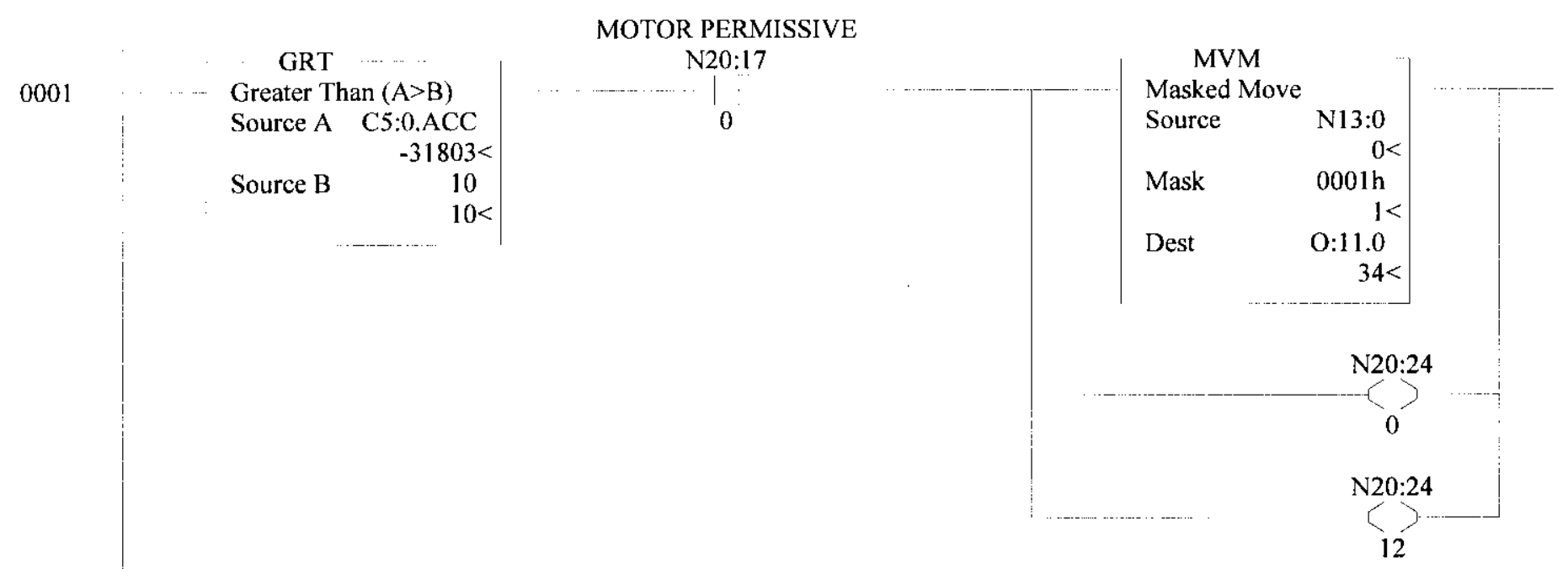

\section{Major}

Error

Fault

Code

$$
\begin{aligned}
& \text { Equal } \\
& \text { Source A }
\end{aligned}
$$

Source B
Overflow

Trap

$$
\text { S:5 }
$$$$
0
$$

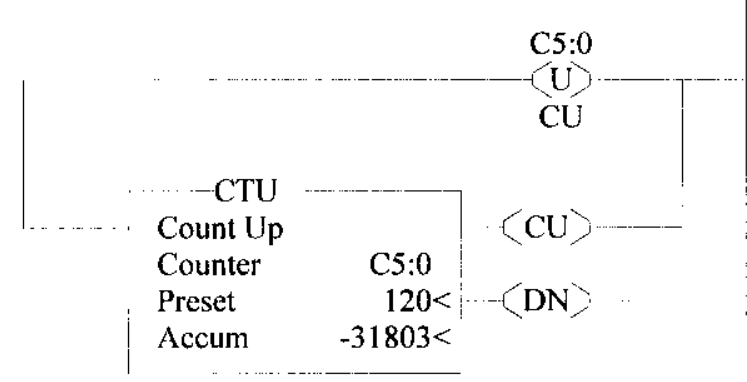

MOTOR PERMISSIVE

MOTOR PERMISSIVE N20:17

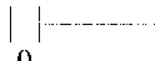

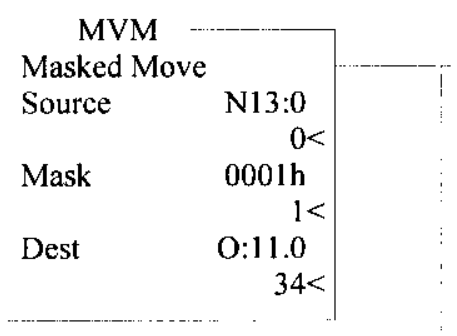

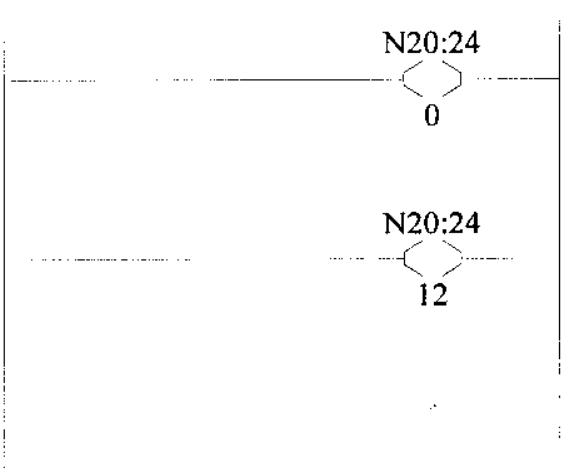


RPP-5492

U109NEW1R1 PIC SKID N

REVISION 0

APPENDIX B
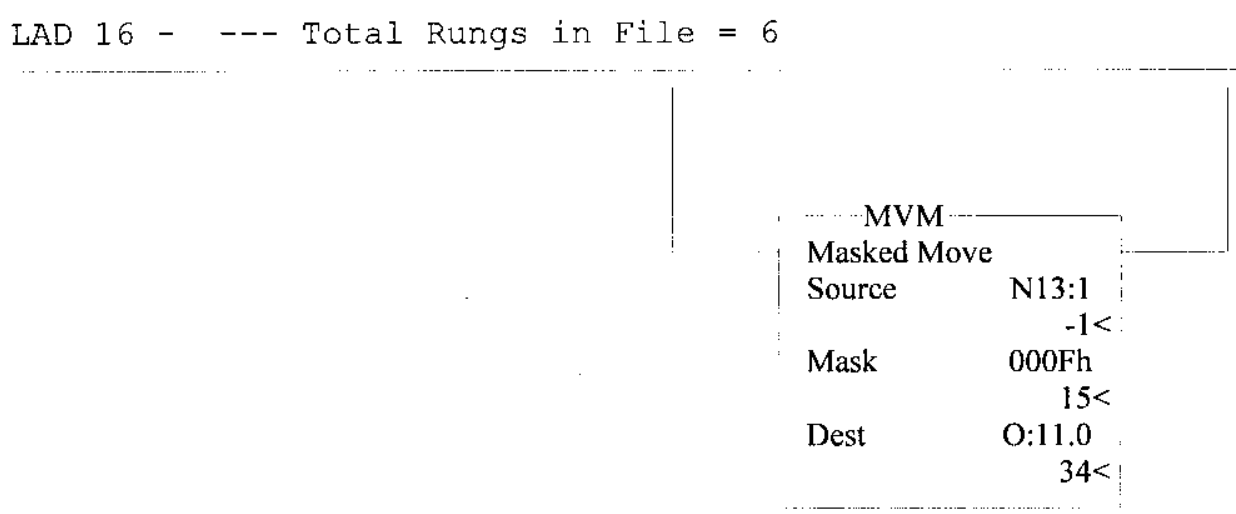

Overflow

Trap

0003

S:5

0

0004

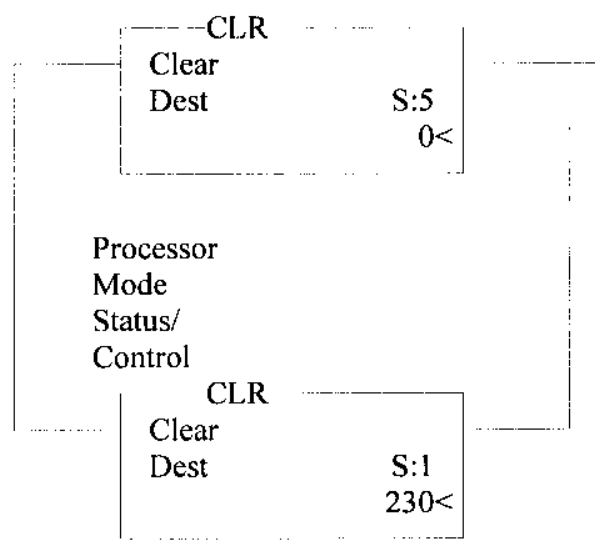

RET

Return

0005

- $\langle$ END $\rangle$

$08: 14: 27$ PAge 2 Wednesday, March 15, 2000PAGE B 79 OF B 90 
LAD 17 - -- Total Rungs in File $=65$

PXPT LOW

N12:0

${ }_{1}$
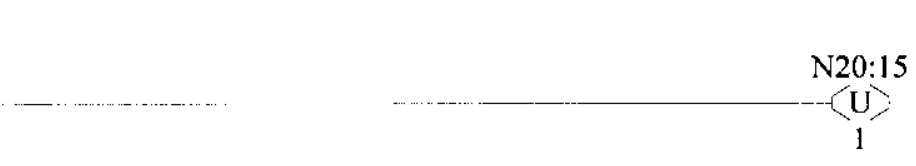

PXPT HIGH

ALARM 2

N12:0

0001

2

N20:15

(U)

JFPT(PS-2)

N12:0

0002

13

$\mathrm{N} 20: 15$

(U)

PUMP TROUBLE

0003

$$
\text { N12:0 }
$$

4

N20:15

(U)

JR-1 FLUSH POS

$$
\text { N12:0 }
$$

0004

5

$20: 15$

(U)

5

PIT LEAK

N12:0

0005

6

N20:15

(U)

PIT LEAK DET. TRB

$$
\text { N12:0 }
$$

0006

7

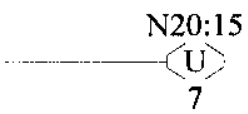

JUMPER HT TRB

N12:0

0007

$$
8
$$

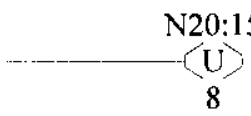

WATER TK LOW LEVEL

0008

$$
\text { N12:0 }
$$

9

N20:15

(U)

INSTR ENCL HI TEMP

$$
\text { N12:0 }
$$

0009

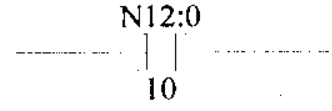

AIR COMPR HI TEMP

0010

N12:0

11

$\mathrm{N} 20 \cdot 15$

(U)

PUMP SHUTDOWN

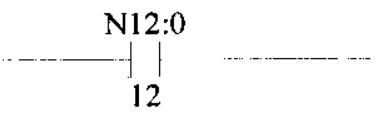

N20:15

U

12

0011

12 
LAD 17 - -- Total Rungs in File $=65$

SALTWELL LOW LEVEL

0012

N12:0

$\mathrm{N} 20 \cdot 15$

(U)

13

13

RFPT SIGNAL LOSS

RFPT SIG LOSS ALARM N12:0 N20:15

0013 14

(U)

DH485 FAILURE

$$
\text { N12:0 }
$$

0014

15

JFPT SIGNAL LOSS

N12:1

0015

0

(20:15

U

JFPT SIG LOSS ALARM N20:16

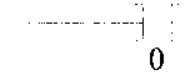

OTHER U LEAK ALARMS
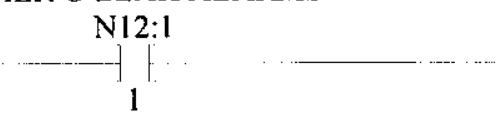

0016

COB U29 LEAK

0017

N12:1

$-1$

$$
2
$$

(U)

2

COB U29 DET TRB

N12:1

0018

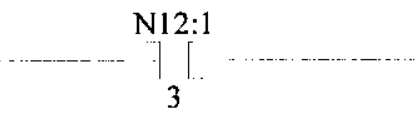

$\mathrm{N} 20: 16$

1

OTHER U SKID IN

FLUSH

N12:1

0019<smiles>[C]1C[As]C1</smiles>

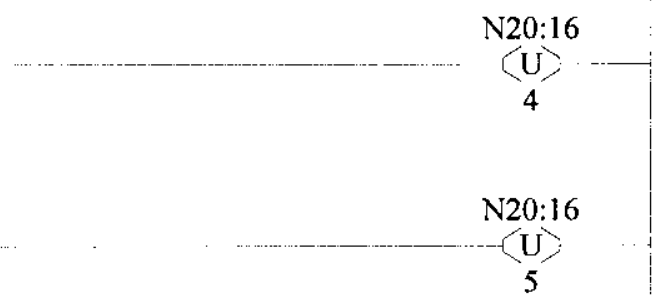

FGM HI LFL

$$
\mathrm{N} 12: 1
$$

0021

6

N20:16

LEAK STATION 4 TRB

0022

N12:1

7

$\mathrm{N} 20: 16$ 
LAD 17 - --- Total Rungs in File $=65$

\begin{tabular}{c|c} 
& $\begin{array}{c}\text { OTHER U SKID } \\
\text { SHUTDOWN } \\
\text { N12:1 }\end{array}$ \\
0023 & $\vdots$ \\
$\mathbf{8}$
\end{tabular}

CGM HI LFL

$0024 \quad$ N12:1

VALVE PIT U-C LEAK

0025 N12:1

10

VALVE PIT U.C TRB

0026

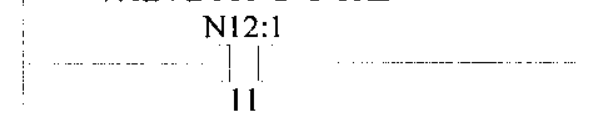

VALVE PIT U-D LEAK

0027

N12:1

12

VALVE PIT U-D TRB N12:1

0028

13

CGM FAULT

N12:1

0029

14

LEAK STATION 5 TRB

0030

N12:1

15

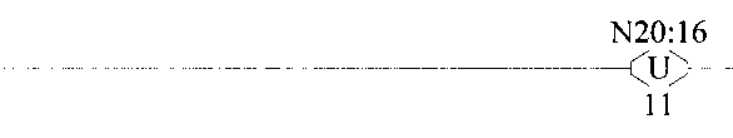

N20:16

(U)

12

N20:16

(U)

13

N20:16

(U)

14

$\mathrm{N} 20: 16$

(U)

15

N20:15

(U)

\begin{tabular}{c}
$\mathrm{N} 12: 0$ \\
\hdashline \\
0
\end{tabular}

COB U-33 LEAK

N12:2

0032

0

N20:55

(U)

DILUTION TK NO FLOW

N12:2

0033

2

$\mathrm{N} 20: 55$

(U)

2

COB U-33 TRB

$\mathrm{N} 12: 2$

3 
LAD 17 - -- Total Rungs in File $=65$

APPENDIX B

OTHER U SKID HI

FLUSH PRESS

0035

$\mathrm{N} 12: 2$

4

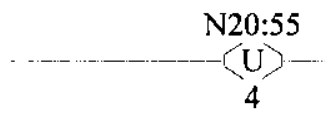

OTHER U SKIDS HI

RECIRC PRESS

0036

$\mathrm{N} 12: 2$

5

N20:55

(U)

RECIRC HIGH PRESS

0037

N12:2

N20:55

6

- (U)

6

VALVE PIT U-A LEAK N12:2

0038

7

N20:55

(U)

7

VALVE PIT U-A TRB

0039

N12:2

N20:55

(U)

8

VALVE PIT U-B LEAK N12:2

$\mathrm{N} 20: 55$

0040 $9^{1}$

VALVE PIT U-B TRB

0041 N12:2

10

COB U-30 LEAK

$\mathrm{N} 12: 2$

0042

11

N20:55

(U)

COB U-30 TRB

N12:2

0043

12

N20:55

12

OTHER U SKID RFPT

SIGNAL LOSS

$\mathrm{N} 12: 2$

0044

13

N20:55

- U

OTHER U SKID JFPT

SIGNAL LOSS

0045 N12:2

14 
RPP-5492

LAD 17 - -- Total Rungs in File $=65$

0046

VALVE PIT SY-B LEAK

N12:2

15

$\mathrm{N} 20: 55$

(U)

15

WATER CAB TEMP LOW

$\mathrm{N} 12: 3$

0047

0

N20:56

(U)

WFIE CAB TEMP LOW N12:3

0048

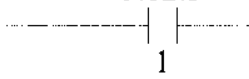

N20:56

(U)

N12:3

0049

i

N20:56

(U)

N12:3

0050

$\left.\right|_{3}$

N20:56

$$
3
$$

EXHAUSTER ON LINE

0051

N12:3

4

N20:56

4

N12:3

N20:56

(U)

5

5

N12:3

0053

$-1+$

N20:56

(U)

N12:3

N20:56

(U)

7

N12:3

N20:56

0055

8

(U)

N12:3

0056

9

N20:56

(U)

9

N12:3

0057

10

N20:56

(U)

N12:3

0058<smiles>[Tl]C1CCCC1</smiles>

$\mathrm{N} 20: 56$

11

(U)

N20:56

(U)

12 
RPP-5492

U109NEW1R1 PIC SKID N

REVISION 0

APPENDIX B

LAD 17 - -- Total Rungs in File $=65$

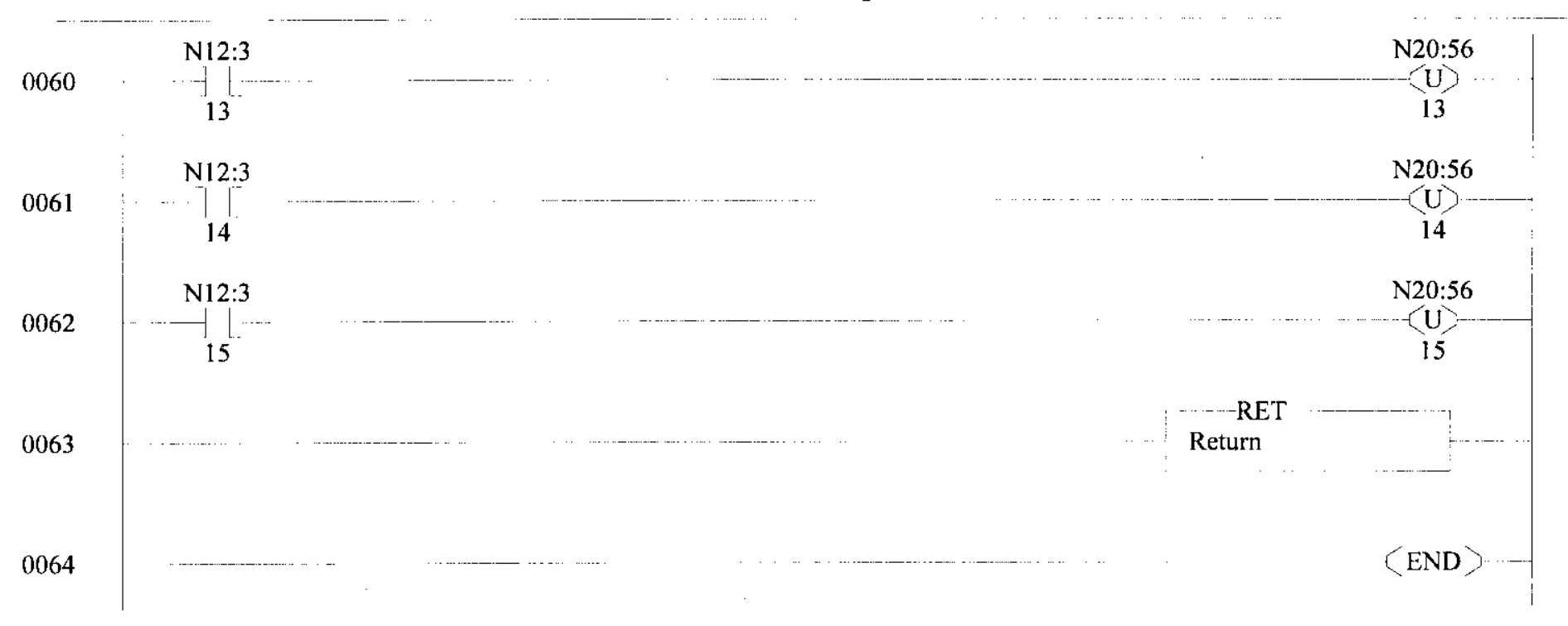

08:14:28 PAGE 6 wednesday, March 15, 2000PAGE B 86 OF B 90 
RPP-5492

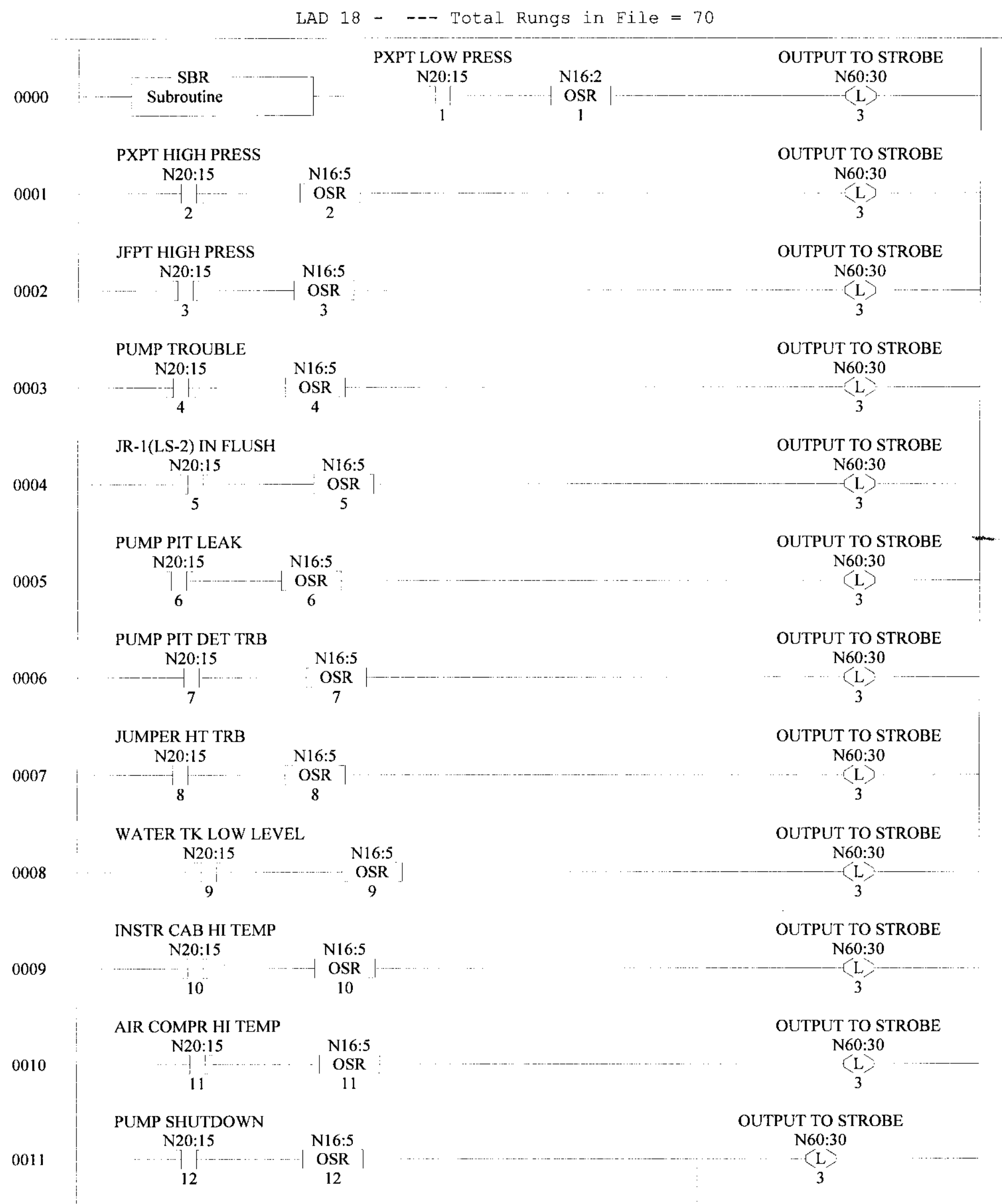


PUMP SHUTDOWN

N20:15
N16:5 U109NEW1R1 PIC SKID N

LAD 18 - -- Total Rungs in File $=70$
OUTPUT TO STROBE RPP-5492

N60:30 REVISION 0

APPENDIX B

0012

SALTWELL LOW LEVEL

\begin{tabular}{c|c}
$\mathrm{N} 20: 15$ & $\begin{array}{c}\mathrm{N} 16: 5 \\
\mathrm{OSR} \\
13\end{array}$ \\
13 &
\end{tabular}

RFPT SIG LOSS ALARM $\mathrm{N} 20: 15$

0013

14

$$
\text { N16:5 }
$$

OSR

14

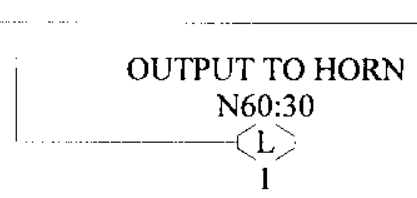

DH485 COMM FAILURE

0014 $\mathrm{N} 20: 15$

15

JFPT SIG LOSS ALARM

0015

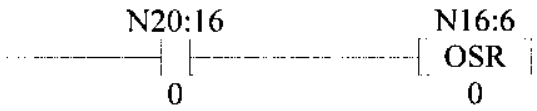

OTHER U SKID LEAKS

0016

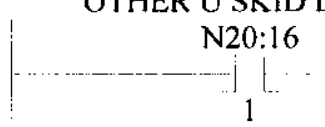

COB U-29 LEAK

0017

0018<smiles>[Mg][Mg][Mg]</smiles>

2

N16:6

2

N16:5

OSR

15

OUTPUT TO STROBE

$\mathrm{N} 60: 30$

3

OUTPUT TO STROBE

N60:30

(L)

3

OUTPUT TO STROBE N60:30

(L)

OUTPUT TO STROBE $\mathrm{N} 60: 30$

(L)

3

OUTPUT TO STROBE N60:30

N16:9
OSR

10

(L)

OUTPUT TO STROBE

N60:30

(L)

COB U-29 DET TRB N20:16

3

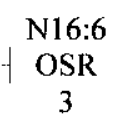

OUTPUT TO STROBE N60:30

(L)

OTHER U SKIDS IN

FLUSH POS.

0019
$\mathrm{~N} 20: 16$

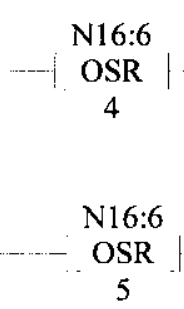

RECIRC LOW FLOW

$\begin{array}{cc}\text { N20:16 } & \text { N16:6 } \\ 5 & \text { OSR }\end{array}$

FGM HIGH LFL

0021

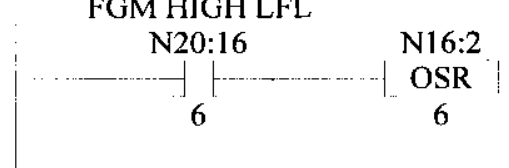

OUTPUT TO STROBE N60:30

(L)

OUTPUT TO STROBE N60:30

(L)

OUTPUT TO STROBE N60:30

3 
LAD 18 - -- Total Rungs in File $=70$

0022

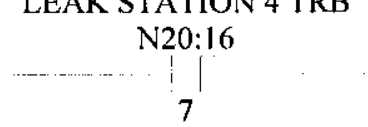

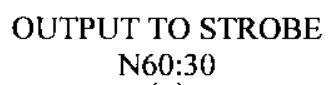

OUTPUT TO STROBE N60:30

OUTPUT TO STROBE N60:30

$$
\begin{gathered}
\text { N16:9 } \\
\text { OSR } \\
11
\end{gathered}
$$

OSR

7

- (L)

3

OUTPUT TO STROBE $\mathrm{N} 60: 30$

(L)

OUTPUT TO STROBE N60:30<smiles>C1=C[As]2C=C[C]12</smiles>

10

OUTPUT TO STROBE N60:30

(L)

OUTPUT TO STROBE N60:30

(L)

OUTPUT TO STROBE N60:30

N16:7

OSR
0

3

OUTPUT TO STROBE N60:30

(L)

3

OUTPUT TO STROBE N60:30

(L)

OUTPUT TO STROBE N60:30

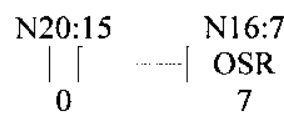

COB U-33 LEAK N20:55

N16:7

N16:9

14
L

OUTPUT TO STROBE N60:30

(L) 


\begin{tabular}{|c|c|c|c|}
\hline 0033 & \begin{tabular}{cc}
$\mathrm{N} 20: 55$ & $\mathrm{~N} 16: 7$ \\
\hdashline & $\mathrm{OSR}$ \\
1 & 11
\end{tabular} & ...... & $\begin{array}{c}\text { OUTPUT TO STROBE } \\
\text { N60:30 } \\
\text { (L) } \\
3\end{array}$ \\
\hline 0034 & $\begin{array}{cc}\text { DILUTION NO FLOW } \\
\text { N20:55 } & \text { N16:7 } \\
2 & \text { OSR } \\
& 12\end{array}$ & & $\begin{array}{c}\text { OUTPUT TO STROBE } \\
\text { N60:30 } \\
-(\mathbf{L}) \\
3\end{array}$ \\
\hline 0035 & \begin{tabular}{lc} 
COB U-33 TRB & \\
N20:55 & N16:7 \\
\hdashline 3 & OSR $\mid-13$
\end{tabular} & $\ldots \ldots$ & $\begin{array}{c}\text { OUTPUT TO STROBE } \\
\text { N60:30 } \\
\qquad \mathrm{L} \\
3\end{array}$ \\
\hline 0036 & $\begin{array}{l}\text { OTHER U SKIDS HI } \\
\text { FLUSH PRESS } \\
\text { N20:55 } \\
\left.\right|_{4} ^{\mid}\end{array}$ & & $\begin{array}{c}\text { OUTPUT TO STROBE } \\
\text { N60:30 } \\
\mathbf{3}\end{array}$ \\
\hline 0037 & $\begin{array}{l}\text { OTHER U SKIDS HI } \\
\text { RECIRC PRESS } \\
\qquad \begin{array}{c|c}\text { N20:55 } & \text { OSR } \\
\hdashline 5 & 15\end{array}\end{array}$ & $\ldots \ldots$ & $\begin{array}{c}\text { OUTPUT TO STROBE } \\
\text { N60:30 } \\
3 \\
1\end{array}$ \\
\hline 0038 & $\begin{array}{cc}\text { RECIRC HIGH PRESS } \\
\qquad 20: 55 & \text { N16:8 } \\
& \text { OSR }\end{array}$ & & $\begin{array}{c}\text { OUTPUT TO STROBE } \\
\text { N60:30 } \\
\text { L } \\
3\end{array}$ \\
\hline 0039 & 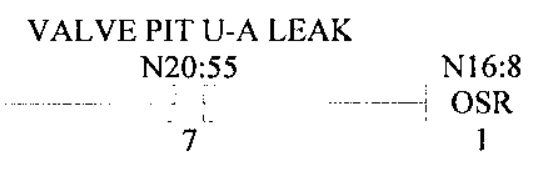 & . & $\begin{array}{l}\text { OUTPUT TO STROBE } \\
\text { N60:30 } \\
(\mathrm{L}) \\
3\end{array}$ \\
\hline 0040 & $\begin{array}{cc}\text { VALVE PIT U-A TRB } \\
\text { N20:55 } \\
\qquad \begin{array}{c}\text { N16:8 } \\
8\end{array} & 2\end{array}$ & & $\begin{array}{c}\text { OUTPUT TO STROBE } \\
\text { N60:30 } \\
3 \\
-(L)-\end{array}$ \\
\hline 0041 & $\begin{array}{cc}\text { VALVE PIT U-B LEAK } \\
\text { N20:55 } \\
9\end{array}$ & 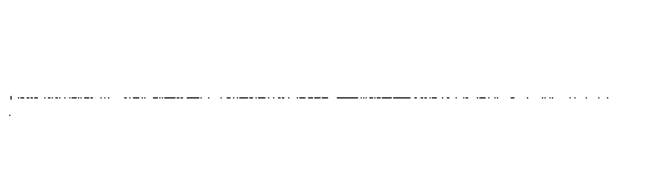 & $\begin{array}{c}\text { OUTPUT TO STROBE } \\
\text { N60:30 } \\
\langle(L) \\
3\end{array}$ \\
\hline 0042 & $\begin{array}{cc}\text { VALVE PIT U-B TRB } \\
\qquad 10 & \text { N16:8 } \\
10: 55 & 4\end{array}$ & $\ldots \ldots$ & $\begin{array}{c}\text { OUTPUT TO STROBE } \\
\text { N60:30 } \\
\qquad(L)-(-)\end{array}$ \\
\hline 0043 & $\begin{array}{l}\text { COB U-30 LEAK } \\
\prod_{11}^{N 20: 55} \\
\begin{array}{c}\text { OSR } \\
5\end{array}\end{array}$ & & $\begin{array}{c}\text { OUTPUT TO STROBE } \\
\text { N60:30 } \\
\mathbf{L}> \\
3\end{array}$ \\
\hline
\end{tabular}


RPP-5492

LAD 18 - -- Total Rungs in File $=70$

0044

0045

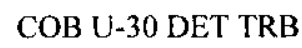

COB U-30 DET TRB

N20:55

12

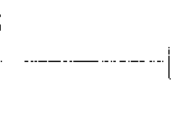

N16:8

6

OTHER U SKIDS RFPT

SIGNAL LOSS

N20:55

13

N16:8

OSR

7

OTHER U SKIDS JFPT

SIGNAL LOSS

0046

N20:55

14

VALVE PIT SY-B LEAK

0047

N20:55

15

WATER CAB LOW TEMP

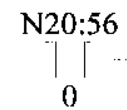

0048

0049

0050

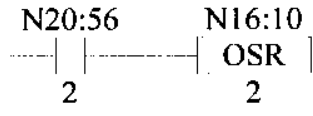

WFIE CAB LOW TEMP

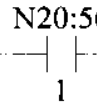

N16:8

OSR

8

N16:9

OSR

15

N16:10

OSR

0

N16:10
OSR
1

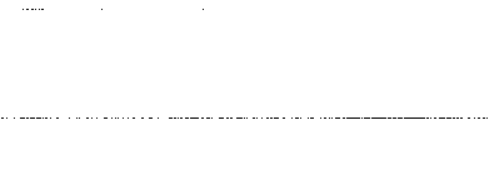

(2)


LAD 18 - - Total Rungs in File $=70$

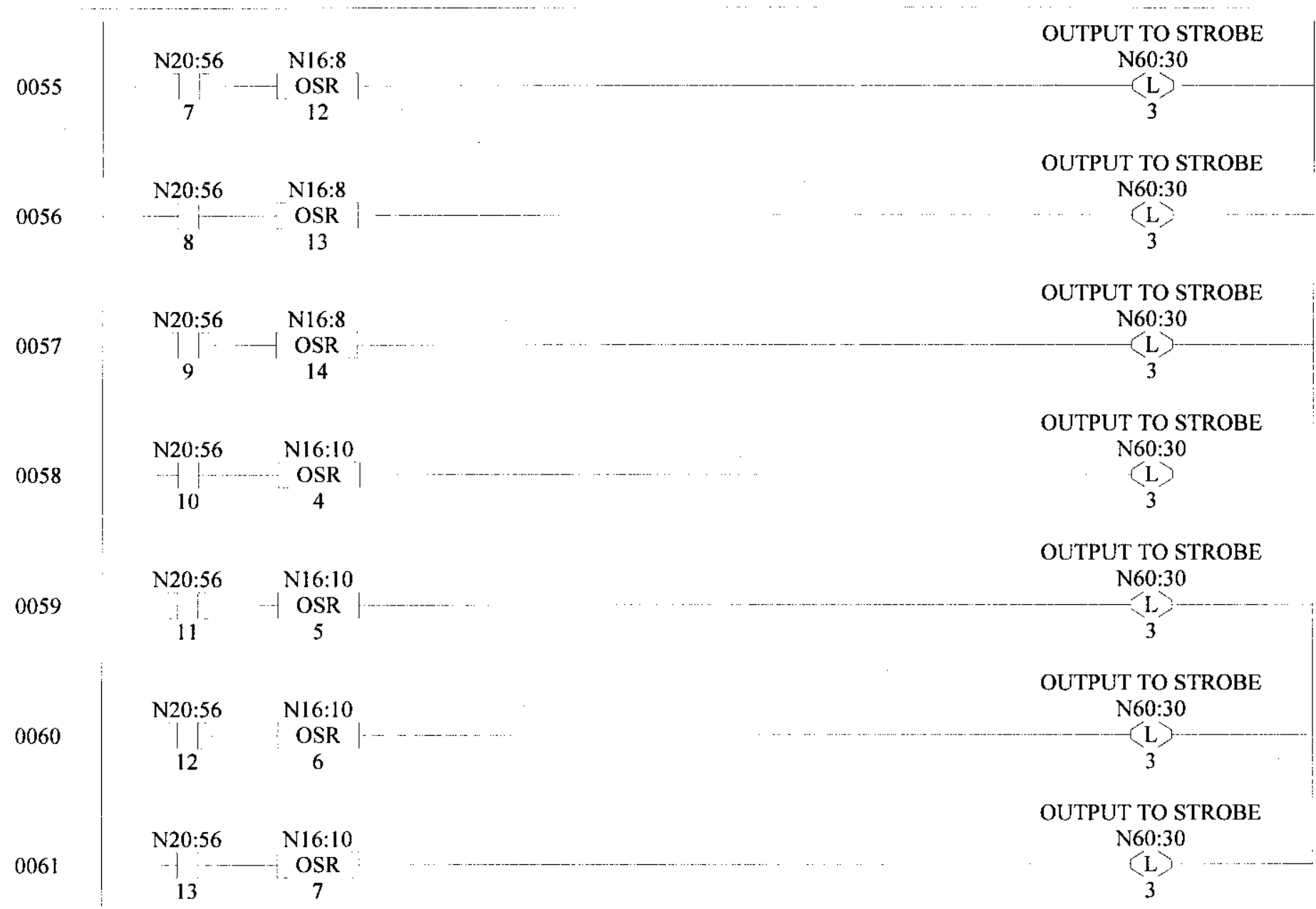

0062

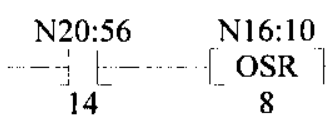

OUTPUT TO STROBE

0062

0063

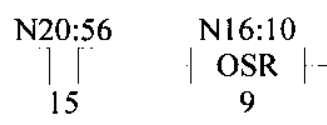

OUTPUT TO STROBE

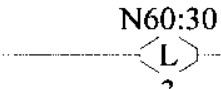

3

3

AMBER LIGHT ON WHEN PXPT LOW PRESS

CHECK IF PXPT IS

LESS THAN 15PSIG

$0064 \quad$ Less Than $(\mathrm{A}<\mathrm{B})$

Source A N60:18

$\begin{array}{ll} & 3352 \\ \text { Source B } & 3932\end{array}$

OUTPUT TO AMBER LAMP N60:31 
LAD 18 - -- Total Rungs in File $=70$

\section{APPENDIX B}

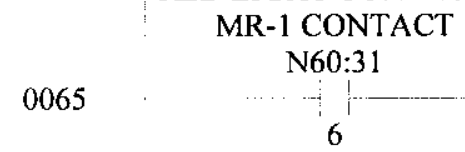

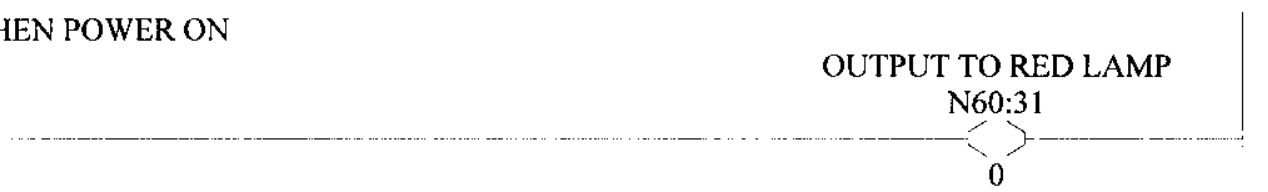

\section{OUTPUT TO BLUE LAMP} N60:31

$$
3
$$

\section{OUTPUT TO GREEN LAMP} N60:31<smiles>C1CSC1</smiles>

RET

Return 
RPP-5492

LAD 19 - -.. Total Rungs in File $=8$

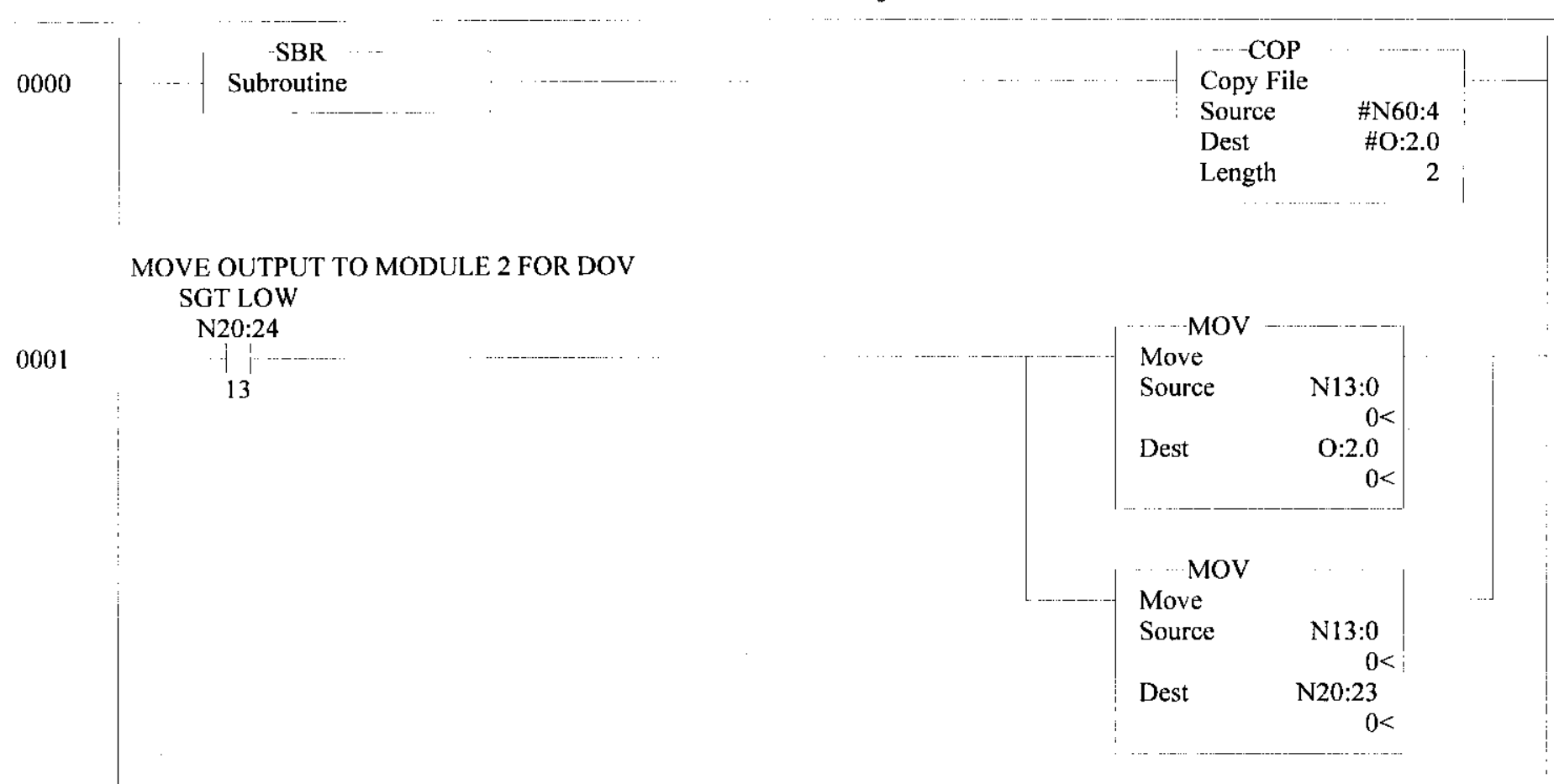

MOVE OUTPUTS TO MODULE 8

0002

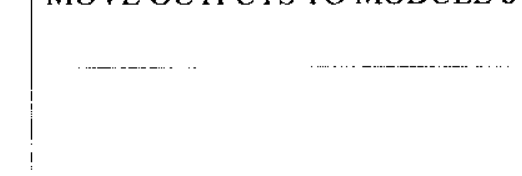

\begin{tabular}{lc}
\multicolumn{2}{c}{ MVM } \\
Masked Move \\
Source & N60:28 \\
& $1280<$ \\
Mask & $003 \mathrm{Fh}$ \\
& $63<$ \\
Dest & $0: 8.0$ \\
& $0<$
\end{tabular}

MOVE OUTPUTS TO MODULE 9
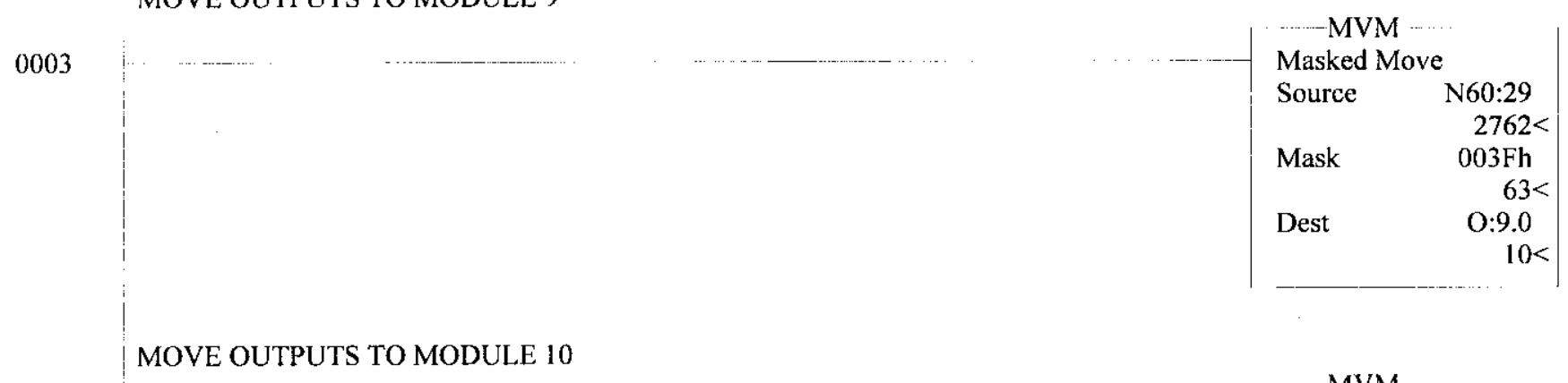

0004

MOVE OUTPUTS TO MODULE 10

\begin{tabular}{lc}
\multicolumn{2}{l}{ Masked Move } \\
Source & N60:30 \\
& $1280<$ \\
Mask & $003 \mathrm{Fh}$ \\
& $63<$ \\
Dest & $\mathrm{O}: 10.0$ \\
& $0<$
\end{tabular}


RPP-5492

U109NEW1RI PIC SKID N

REVISION 0

APPENDIX B

LAD 19 - ... Total Rungs in File $=8$

MOVE OUTPUTS TO MODULE 11

0005
0006

MVM

Masked Move

Source

Mask

Dest

RET

Return

0007 


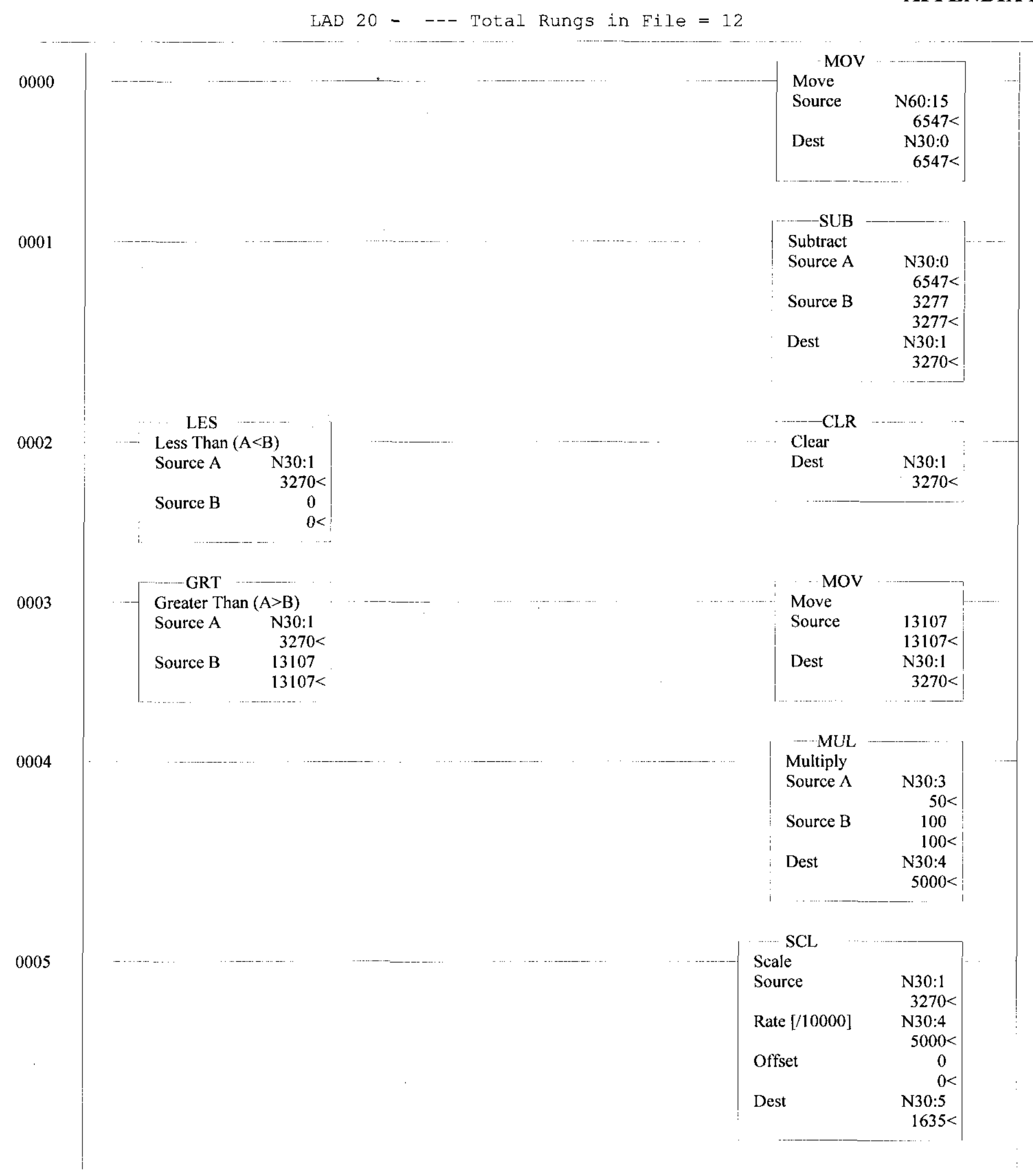


LAD 20 - - Total Rungs in File $=12$

\begin{tabular}{l|ll}
0006 & \multicolumn{2}{|c}{ GRT } \\
Greater Than $(\mathrm{A}>\mathrm{B})$ \\
Source A & N30:5 \\
& $1635<$ \\
Source B & 4096 \\
& $4096<$
\end{tabular}

$\begin{array}{lc}\text { Move } & \\ \text { Source } & 4095 \\ & 4095< \\ \text { Dest } & N 30: 5 \\ & 1635<\end{array}$

SCALE DILUTION UNIT(GPM) TO PLC OUTPUT UNIT (6242 - 31208) 4-20mA

0007

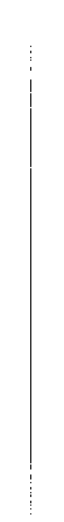

\begin{tabular}{|lc|}
$\mid \begin{array}{l}|c| \\
\text { SCP }\end{array}$ \\
Input & \\
& N30:Parameters \\
Input Min. & $1635<$ \\
& 41 \\
& $41<$ \\
Input Max. & 4095 \\
& $4095<$ \\
Scaled Min. & 6242 \\
& $6242<$ \\
Scaled Max. & 31208 \\
& $31208<$ \\
Output & N30:2 \\
& $16058<$ \\
\hline
\end{tabular}

IF CALCULATED DILUTION OUTPUT IS LESS THAN THE MINIMUM FLOW INPUT FROM THE DTAM, THEN FORCE DILUTION OUTPUT TO BE THE MINIMUM FLOW VAIUUE

0008 LES

Less Than $(A<B)$

Source A N30:2

$\begin{array}{ll} & 16058< \\ \text { Source B } & \text { N30:6 }\end{array}$

$6240<$

0009

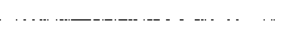

$\begin{array}{|lc|}\text { Move } & \\ \text { Source } & \text { N30:6 } \\ & 6240< \\ \text { Dest } & \text { N30:2 } \\ & 16058<\end{array}$

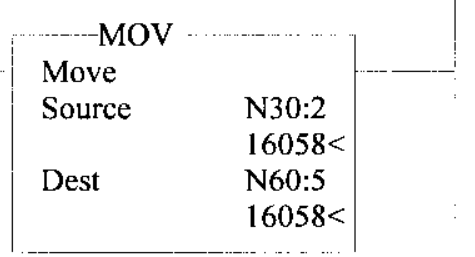

0010

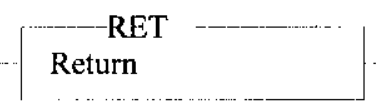

0011 


\section{RPP-5492 REVISION 0, APPENDIX C}

Mar 1500

FILE: U109NEW1. CFG

PAGE

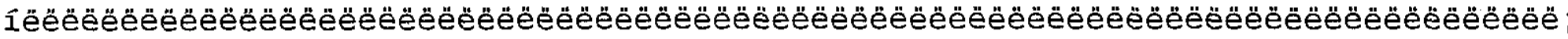

$\circ$

$\circ$

$\circ$

$\circ$

a

- SLC TYPE:

PROGRAM MEMORY USED:

NUMBER OF SCREENS USED:
DTAM Plus

PROGRAM FILE DOCUMENTATION

Allen-Bradley Company, Inc.

$\circ$

- UPDATE DELAY:

- MASTER SECURITY CODE:

- COM pORT PARAMETERS :

- PRINTER PORT PARAMETERS:

a

0

$\circ$

o

$\circ$

0

$\circ$$$
\text { : }
$$

$$
\text { ááa }
$$




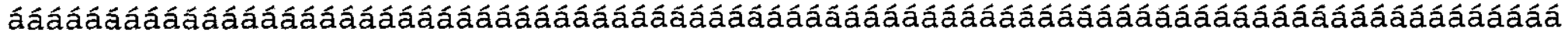

BACKGROUND REGISTER 1: BIT DATA

MONITOR REGISTER NUMBER: N20:15

BIT\# ALARM STATE GOTO SCREEN\# BIT\# ALARM STATE GOTO SCREEN\#

$\begin{array}{llllll}0 & \text { ON (1) } & 100 & 8 & \text { ON (1) } & 108 \\ 1 & \text { ON (1) } & 101 & 9 & \text { ON (1) } & 109 \\ 2 & \text { ON (1) } & 102 & 10 & \text { ON (1) } & 110 \\ 3 & \text { ON }(1) & 103 & 11 & \text { ON (1) } & 111 \\ 4 & \text { ON (1) } & 104 & 12 & \text { ON (1) } & 112 \\ 5 & \text { ON (1) } & 105 & 13 & \text { ON (1) } & 113 \\ 6 & \text { ON (1) } & 106 & 14 & \text { ON (1) } & 114 \\ 7 & \text { ON (1) } & 107 & 15 & \text { ON (1) } & 115\end{array}$

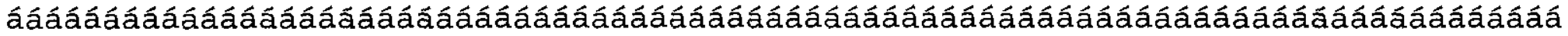
BACKGROUND REGISTER 2: BIT DATA

MONITOR REGISTER NUMBER: N20:16

BIT\# ALARM STATE GOTO SCREEN\# BIT\# ALARM STATE GOTO SCREEN\#

$\begin{array}{llllll}0 & \text { ON (1) } & 116 & 8 & \text { ON (1) } & 124 \\ 1 & \text { ON (1) } & 117 & 9 & \text { ON (1) } & 125 \\ 2 & \text { ON (1) } & 118 & 10 & \text { ON (1) } & 126 \\ 3 & \text { ON (1) } & 119 & 11 & \text { ON (1) } & 127 \\ 4 & \text { ON (1) } & 120 & 12 & \text { ON (1) } & 128 \\ 5 & \text { ON (1) } & 121 & 13 & \text { ON (1) } & 129 \\ 6 & \text { ON (1) } & 122 & 14 & \text { ON (1) } & 131 \\ 7 & \text { ON (1) } & 123 & 15 & \text { ON (1) } & 132\end{array}$

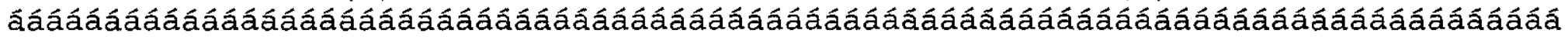
BACKGROUND REGISTER 3: BIT DATA

MONITOR REGISTER NUMBER: N20:55

BIT\# ALARM STATE GOTO SCREEN\# BIT\# ALARM STATE GOTO SCREEN\#

$\begin{array}{llllll}0 & \text { ON (1) } & 133 & 8 & \text { ON (1) } & 141 \\ 1 & \text { ON (1) } & 134 & 9 & \text { ON (1) } & 142 \\ 2 & \text { ON (1) } & 135 & 10 & \text { ON (1) } & 143 \\ 3 & \text { ON (1) } & 136 & 11 & \text { ON (1) } & 144 \\ 4 & \text { ON (1) } & 137 & 12 & \text { ON (1) } & 145 \\ 5 & \text { ON (1) } & 138 & 13 & \text { ON (1) } & 146 \\ 6 & \text { ON (1) } & 139 & 14 & \text { ON (1) } & 147 \\ 7 & \text { ON (1) } & 140 & 15 & \text { ON (1) } & 148\end{array}$

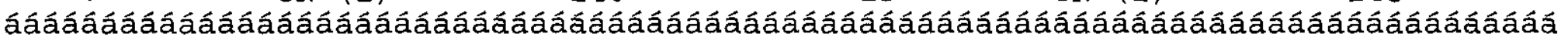
BACKGROUND REGISTER 4 : BIT DATA

MONITOR REGISTER NUMBER: N20:56

BIT\# ALARM STATE GOTO SCREEN\# BIT\# ALARM STATE GOTO SCREEN\#

$\begin{array}{llllcl}0 & \text { ON (1) } & 149 & 8 & \text { ON (1) } \\ 1 & \text { ON (1) } & 150 & 9 & \text { THIS BIT IS NOT MONITORED } \\ 2 & \text { ON }(1) & 151 & 10 & \text { THIS BIT IS NOT MONITORED } \\ 3 & \text { ON (1) } & 152 & 11 & \text { THIS BIT IS NOT MONITORED } \\ 4 & \text { ON (1) } & 153 & 12 & \text { THIS BIT IS NOT MONITORED } \\ 5 & \text { ON }(1) & 1 \text { PAGE C 2 OF } & \end{array}$


Mar 1500

FILE : U109NEW1. CFG

PAGE 3

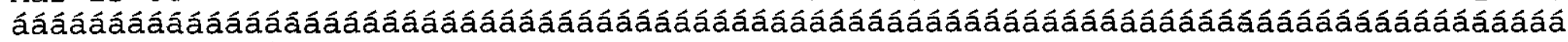
SCREEN\# TYPE

001

MAIN MENU

SCREEN\#

010

SUB-MENU

116

TYPE

011

012

DATA ENTRY

117

SUB-MENU

118

013

014

015

016

017

018

019

020

021

022

023

024

025

026

027

028

029

030

031

032

033

034

035

036

037

040

041

042

043

044

045

046

048

050

052

053

054

055

056

057

058

DATA DISPLAY

120

DATA ENTRY

121

DATA ENTRY

122

SECUR ITY

123

SUB-MENU

124

DATA ENTRY 125

DATA ENTRY 126

DATA DISPLAY 127

DATA ENTRY

128

DATA ENTRY

129

DATA ENTRY

130

DATA DISPLAY 131

DATA DISPLAY 132

DATA DISPLAY 133

DATA DISPLAY 134

DATA DISPLAY 135

DATA DISPLAY

136

SUB-MENU

137

DATA ENTRY

138

DATA ENTRY

139

DATA DISPLAY

SUB-MENU

140

141

DATA ENTRY 142

DATA ENTRY 143

DATA DISPLAY 144

SUB-MENU

DATA DISPLAY

145

DATA DISPLAY

146

DATA DISPLAY

147

DATA DISPLAY

148

149

DATA DISPLAY

150

DATA DISPLAY 151

DATA DISPLAY 152

DATA DISPLAY 153

DATA DISPLAY 154

DATA DISPLAY 155

DATA DISPLAY 156

DATA DISPLAY 157

DATA DISPLAY 158

DATA DISPLAY 159

DATA DISPLAY 200

ALARM

ALARM

ALARM

ALARM

ALARM

ALARM

ALARM

ALARM

ALARM

ALARM

ALARM

ALARM

ALARM

ALARM

ALARM

ALARM

ALARM

ALARM

ALARM

ALARM

ALARM

ALARM

ALARM

ALARM

ALARM

ALARM

ALARM

ALARM

ALARM

ALARM

ALARM

ALARM

ALARM

ALARM

ALARM

ALARM

ALARM

ALARM

ALARM

ALARM

ALARM

ALARM

ALARM

ALARM

100 ALARM

101 ALARM

102 ALARM

103 ALARM

104 ALARM

105 ALARM

106 ALARM

107 ALARM

108 ALARM

109 ALARM

110 ALARM

111 ALARM

PAGE C 4 OFC 50 
112

113

114

ALARM

ALARM

ALARM

\section{RPP-5492 REVISION 0, APPENDIX C}

PAGE C 5 OF C 50 
Mar 1500

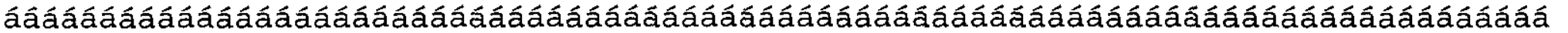

$\begin{array}{ll}\text { P-A/D SECURITY } & \\ \text { SCREEN MEMORY USED: } & 61 \text { BYTES } \\ & \\ \text { PREVIOUS MENU : } & \text { UNLINKED } \\ \text { NEXT SCREEN: } & \text { UNLINKED } \\ \text { PREVIOUS SCREEN: } & \text { UNLINKED } \\ \text { SECURITY CODE \#1: } & 12 \text { ????? } \\ \text { SECURITY CODE \#2: } & 34 \text { ????? } \\ \text { SECURITY CODE \#3: } & 87 ? ? ? ? ? ?\end{array}$

İeëëëëëëëëëëëe̋ëëëëë;

$\circ$ *RESTRICTED ACCESS *०

- ENTER CODE

0

åëëëëëëëëëe̋ëëëëëëëëf

SECURITY CODE \#1: 12??????

$\begin{array}{ll}\text { SECURITY CODE \#2: } & \text { 34?????? } \\ \text { SECURITY CODE \#3: } & \text { 87?????? }\end{array}$

PAGE C 6 OF C 50 
ááááááááááăááááááááááááááááááááááááááááááááááááááááááááááááááááá

SCREEN \#001: MAIN MENU

SCREEN MEMORY USED: 88 BYTES 1́̈ëëëëëëëëëëëëëëëëëë; U-109

1. Pump 4. Alarms ${ }^{\circ}$

$\circ 2$. Util 5. Clear

3. HtTrace 6. Dratio

ฉ̊ëëëëëëëëëëëëëëëëëëë $f$

\begin{abstract}
Item/Key \# 1 links to screen 010
Item/Key \# 2 links to screen 020

Item/Key \# 3 links to screen 030

Item/Key \# 4 links to screen 040
\end{abstract}

\begin{abstract}
Item/Key \# 5 links to screen 023
Item/Key \# 6 links to screen 034

Item/Key \# 7 links to screen 024

Item/Key \# 8 is unlinked
\end{abstract}

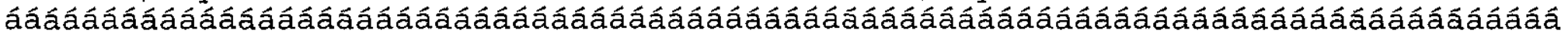

SCREEN \#010: SUB-MENU

SCREEN MEMORY USED: 86 BYTES

PREVIOUS MENU: 001

Item/Key \# 1 links to screen 011

Item/Key \# 2 links to screen 012

Item/Key \# 3 links to screen 013

Item/Key \# 4 links to screen 022 1́ëëëëëëëëëëëëëëëëëëë

-1 Pump On/Off

$\circ 2$ DOV Sub-Menu

-3 CVT, WFT, \& SPGT $\circ$

-4 DOV Manual Output ${ }^{\circ}$

ล̊ëëëëëëëëëëëëëëëëëëë $f$

ááááááááááááááááááááááâááááááăáááááááááááááâááááááááááááááááááăáá

SCREEN \#011: DATA ENTRY

SCREEN MEMORY USED: 98 BYTES

PREVIOUS MENU: $\quad 010$

NEXT SCREEN: 015

PREVIOUS SCREEN: UNLINKED

DATA POSITION \#1: BIT DISPLAY
REGISTER :
$\mathrm{N} 60: 31$
BIT NUMBER:
6
TEXT LENGTH:
3
OFF BIT TEXT:
ON BIT TEXT:
OFF
ON

DATA POSITION \#2: BIT ENTRY

REGISTER: $\quad$ N20:17

BIT NUMBER:
0

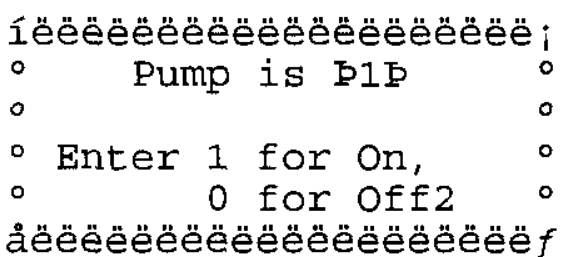

ฉ̊e̊ëëëëëëëëëëëëëëëëëëë $f$

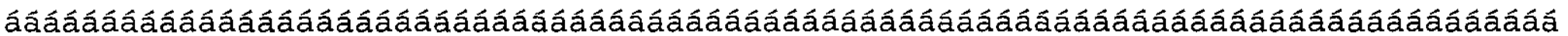

SCREEN \#012: SUB-MENU

SCREEN MEMORY USED: 76 BYTES

PREVIOUS MENU: 010

ENTRY FORMAT:

DEFAULT:

$1 \& 0$ KEYS NONE 


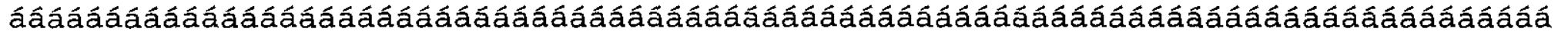

SCREEN \#013: DATA DISPLAY

SCREEN MEMORY USED: 238 BYTES

PREVIOUS MENU: $\quad 010$

NEXT SCREEN : 022

PREVIOUS SCREEN : UNLINKED

DATA POSITION \#I: 16 BIT SIGNED INTEGER DISPLAY 1́ëëëëëëëëëëëëëëëëëë ${ }^{\circ} \mathrm{DOV}$ DS1D $\div$ इ2ए $3 \circ$ - (CVT) DS4DD gpm

-WFT DD5DE in

- SPGR : DDD6DD in åëëëëëëëëëëëëëëëëëëë $f$
REGISTER: $\quad$ N20:23

DECIMAL RIGHT: 0

DECIMAL LEFT: $\quad 3$

SHOW SIGN

LEADING ZEROS NOT SHOWN
REGISTER LOW: $\quad 0$

REGISTER HIGH : $\quad 16383$

DISPLAY LOW: 0

DISPLAY HIGH: $\quad 100$

DATA POSITION \#2: BIT DISPLAY

$\begin{array}{llll}\text { REGISTER : } & \text { N60:31 } & \text { OFF BIT TEXT: } & \text { Off } \\ \text { BIT NUMBER : } & 6 & \text { ON BIT TEXT: } & \text { On } \\ \text { TEXT LENGTH : } & 3 & \end{array}$

DATA POSITION \#3: BIT DISPLAY

$\begin{array}{llll}\text { REGISTER : } & \text { N20:17 } & \text { OFF BIT TEXT: } & \text { M } \\ \text { BIT NUMBER : } & 6 & \text { ON BIT TEXT: } & \text { A } \\ \text { TEXT LENGTH : } & 1 & & \end{array}$

DATA POSITION \#4: 16 BIT SIGNED INTEGER DISPLAY

$\begin{array}{llll}\text { REGISTER : } & \text { N60:15 } & \text { REGISTER LOW: } & 3277 \\ \text { DECIMAL RIGHT: } & 3 & \text { REGISTER HIGH: } & 16384 \\ \text { DECIMAL LEFT: } & 1 & \text { DISPLAY LOW: } & 0.000 \\ \text { NO SIGN PLACE } & & \text { DISPLAY HIGH: } & 8.000\end{array}$

DATA POSITION \#5: 16 BIT UNSIGNED INTEGER DISPLAY

$\begin{array}{llll}\text { REGISTER : } & \text { N60:13 } & \text { REGISTER LOW: } & 3277 \\ \text { DECIMAL RIGHT: } & 1 & \text { REGISTER HIGH: } & 16384 \\ \text { DECIMAL LEFT: } & 3 & \text { DISPLAY LOW: } & 0.0 \\ \text { NO SIGN PLACE } & & \text { DISPLAY HIGH : } & 500.0\end{array}$

LEADING ZEROS NOT SHOWN

DATA POSITION \#6: 16 BIT UNSIGNED INTEGER DISPLAY

$\begin{array}{llll}\text { REGISTER : } & \text { N60:12 } & \text { REGISTER LOW: } & 3277 \\ \text { DECIMAL RIGHT: } & 2 & \text { REGISTER HIGH: } & 16384 \\ \text { DECIMAL LEFT: } & 3 & \text { DISPLAY LOW : } & 0.00 \\ \text { NO SIGN PLACE } & & \text { DISPLAY HIGH : } & 20.00 \\ \text { LEADING ZEROS NOT SHOWN } & & \end{array}$

PAGE C $\_$OF C $\_$50 


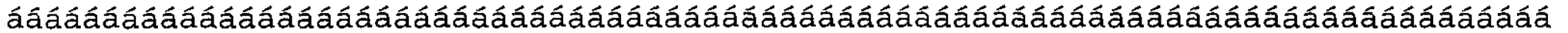

SCREEN \#014: DATA ENTRY

SCREEN MEMORY USED: 207 BYTES

PREVIOUS MENU: 012

NEXT SCREEN: $\quad 001$

PREVIOUS SCREEN : UNLINKED

DATA POSITION \#1: 16 BIT UNSIGNED INTEGER DISPLAY

$\begin{array}{llll}\text { REGISTER : } & \text { N20:23 } & \text { REGISTER LOW: } & 0 \\ \text { DECIMAL RIGHT: } & 0 & \text { REGISTER HIGH : } & 100 \\ \text { DECIMAL LEFT: } & 3 & \text { DISPLAY LOW : } & 0 \\ \text { NO SIGN PLACE } & & \text { DISPLAY HIGH : } & 100\end{array}$

DATA POSITION \#2: 16 BIT UNSIGNED INTEGER DISPLAY

$\begin{array}{llll}\text { REGISTER : } & \text { N60:12 } & \text { REGISTER LOW: } & 3277 \\ \text { DECIMAL RIGHT: } & 2 & \text { REGISTER HIGH: } & 16384 \\ \text { DECIMAL LEFT: } & 3 & \text { DISPLAY LOW: } & 0.00 \\ \text { NO SIGN PLACE } & & \text { DISPLAY HIGH : } & 20.00\end{array}$

DATA POSITION \#3: 16 BIT UNSIGNED INTEGER DISPLAY

$\begin{array}{llll}\text { REGISTER : } & \text { N20:18 } & \text { REGISTER LOW : } & 0 \\ \text { DECIMAL RIGHT: } & 1 & \text { REGISTER HIGH: } & 16383 \\ \text { DECIMAL LEFT: } & 2 & \text { DISPLAY LOW: } & 0.0 \\ \text { NO SIGN PLACE } & & \text { DISPLAY HIGH : } & 20.0 \\ \text { TEATIN ZEROS NOT SHOWN } & & \end{array}$

DATA POSITION \#4: 16 BIT UNSIGNED INTEGER ENTRY

$\begin{array}{llll}\text { REGISTER : } & \text { N20:18 } & \text { REGISTER LOW: } & 0 \\ \text { DECIMAL RIGHT : } & 1 & \text { REGISTER HIGH : } & 16383 \\ \text { DECIMAL LEFT: } & 2 & \text { DISPLAY LOW : } & 0.0 \\ \text { NO SIGN PLACE } & & \text { DISPLAY HIGH: } & 20.0 \\ \text { DEFAULT : } & \text { NONE } & \text { LOW INPUT LIMIT: } & 4.0 \\ & & \text { HIGH INPUT LIMIT: } 9.0\end{array}$

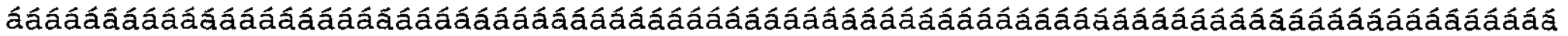

SCREEN \#015: DATA ENTRY

SCREEN MEMORY USED: 114 BYTES

PREVIOUS MENU: $\quad 012$

NEXT SCREEN : 013

PREVIOUS SCREEN : UNLINKED

1̇ëëëëëëëëëëëëëëëëëë ;

- DOV is in DDDIDP.

- Enter 1 for Auto o

- 0 for Manual o

-Enter: 2

åëëëëëëëëëëëëëëëëëë $f$

DATA POSITION \#1: BIT DISPLAY
REGISTER :
$\mathrm{N} 20: 17$
BIT NUMBER:
6
TEXT LENGTH :
6
OFF BIT TEXT: Manual
ON BIT TEXT: Auto

DATA POSITION \#2: BIT ENTRY

PAGE C 9 OF C 50 
REGISTER :

BIT NUMBER :
N2 $0: 17$

6
ENTRY FORMAT :

DEFAULT :
$1 \& 0$ KEYS

NONE

PAGE C 10 OF C 50 


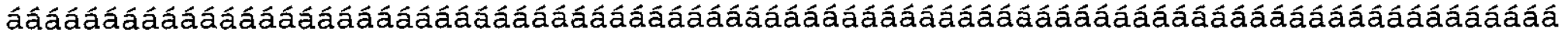
SCREEN \#016: SECURITY
SCREEN MEMORY USED: 61 BYTES
PREVIOUS MENU: 012
NEXT SCREEN: 017
PREVIOUS SCREEN : UNLINKED
SECURITY CODE \#1: 18??????
SECURITY CODE \#2: 22??????
SECURITY CODE \#3: 44 ??????

1́ëëëëëëëëëëëëëëëëëë i

$\circ$ *RESTRICTED ACCESS* 0

$\begin{array}{lll}\circ & \text { ENTER CODE } & \circ \\ \circ & : & \circ\end{array}$

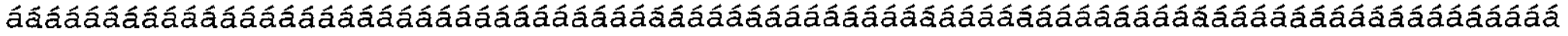

SCREEN \#017: SUB-MENU

\{́ëëëëëëëëëëëëëëëëëè i

SCREEN MEMORY USED: 70 BYTES

PREVIOUS MENU: 012

- DOV PID SUBMENU

${ }^{\circ} 1$ Prop Gain

-2 Reset

-3 Derivative

åëëëëëëëëëëëëëëëëëëë $f$

$\begin{array}{llll}\text { Item/Key \# } & 1 & \text { links to screen } 018 \\ \text { Item/Key \# } & 2 & \text { links to screen } 019 \\ \text { Item/Key \# } 3 & \text { links to screen } 021 \\ \text { Item/Key \# } 4 & \text { is unlinked }\end{array}$

$\begin{array}{lll}\text { Item/Key \# } 5 & \text { is unlinked } \\ \text { Item/Key \# } 6 & \text { is unlinked } \\ \text { Item/Key \# } 7 & \text { is unlinked } \\ \text { Item/Key \# } 8 & \text { is unlinked }\end{array}$

Item/Key \# 5 is unlinked

Item/Key \# 6 is unlinked

Item/Key \# 8 is unlinked

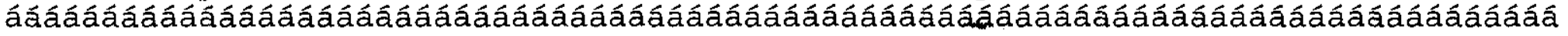

SCREEN \#018: DATA ENTRY

SCREEN MEMORY USED: 185 BYTES

PREVIOUS MENU: $\quad 017$

NEXT SCREEN : 019

PREVIOUS SCREEN : UNLINKED

DATA POSITION \#1: 16 BIT UNSIGNED INTEGER DISPLAY

REGISTER : $\mathrm{N} 20: 23$

DECIMAL RIGHT:

DECIMAL LEFT:

NO SIGN PLACE

LEADING ZEROS NOT SHOWN \{́ëëëëëëëëëëëëëëëëëë ;

- Gain (0.01-327.67) 。

-DOV OUTPUT: D15\%

- Prop. Gain DDL2D50

- Enter DDS350

åëëëëëëëëëëëẹëëëëëëe $f$

DATA POSITION \#2: 16 BIT UNSIGNED INTEGER DISPLAY
REGISTER :
N20 $: 19$
DECIMAL RIGHT: 2
DECIMAL LEFT: 3
REGISTER LOW: 1
REGISTER HIGH : 16383
DISPLAY LOW:
0.01
NO SIGN PLACE
LEADING ZEROS NOT SHOWN

REGISTER LOW: 0

REGISTER HIGH : 16383

DISPLAY LOW :

0

100

DATA POSITION \#3: 16 BIT UNSIGNED INTEGER ENTRY

$\begin{array}{ll}\text { REGISTER : } & \text { N20:19 } \\ \text { DECIMAL RIGHT: } & 2 \\ \text { DECIMAL LEFT: } & 3 \\ \text { NO SIGN PLACE } & \\ \text { DEFAULT : } & \text { NONE }\end{array}$

$\begin{array}{ll}\text { REGISTER LOW : } & 1 \\ \text { REGISTER HIGH : } & 16383 \\ \text { DISPLAY LOW: } & 0.01 \\ \text { DISPIAY HIGH: } & 327.67 \\ \text { LOW INPUT LIMIT: } & 0.01 \\ \text { HIGH INPUT LIMIT: } & 327.67\end{array}$

PAGE C 11 OF C 50 


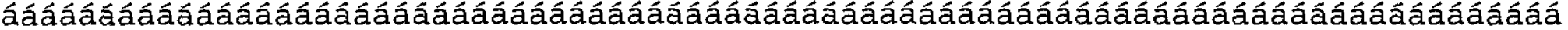

SCREEN \#019: DATA ENTRY

SCREEN MEMORY USED: 177 BYTES

PREVIOUS MENU: 017

NEXT SCREEN : 021

PREVIOUS SCREEN: UNLINKED

DATA POSITION \#1: 16 BIT UNSIGNED INTEGER DISPLAY

$\begin{array}{llll}\text { REGISTER : } & \text { N20:23 } & \text { REGISTER LOW: } & 0 \\ \text { DECIMAL RIGHT: } & 0 & \text { REGISTER HIGH : } & 16383 \\ \text { DECIMAL LEFT: } & 3 & \text { DISPLAY LOW: } & 0 \\ \text { NO SIGN PLACE } & & \text { DISPLAY HIGH : } & 100\end{array}$

乏́ëëëëëëëëëëëëëëëëëë i

${ }^{\circ}$ Reset 0.1 to $327.67 \circ$

${ }^{\circ} \mathrm{DOV}$ OUTPUT: $\$ 1 \mathrm{5}$ - Reset: DDS2DS in/rpo åëëëëëëëëëëëëëëëëëë $f$

LEADING ZEROS NOT SHOWN

DATA POSITION \#2: 16 BIT UNSIGNED INTEGER DISPLAY

$\begin{array}{llll}\text { REGISTER : } & \text { N20:20 } & \text { REGISTER LOW: } & 1 \\ \text { DECIMAL RIGHT: } & 2 & \text { REGISTER HIGH: } & 16383 \\ \text { DECIMAL LEFT: } & 3 & \text { DISPLAY LOW: } & 0.01 \\ \text { NO SIGN PLACE } & & \text { DISPLAY HIGH: } & 327.67 \\ \text { LEADING ZEROS NOT SHOWN } & & \end{array}$

DATA POSITION \#3: 16 BIT UNSIGNED INTEGER ENTRY

$\begin{array}{llll}\text { REGISTER : } & \text { N20:20 } & \text { REGISTER LOW: } & 1 \\ \text { DECIMAL RIGHT: } & 2 & \text { REGISTER HIGH: } & 16383 \\ \text { DECIMAL LEFT : } & 3 & \text { DISPLAY LOW : } & 0.01 \\ \text { NO SIGN PLACE } & & \text { DISPLAY HIGH : } & 327.67 \\ \text { DEFAULT : } & \text { NONE } & \text { LOW INPUT LIMIT : } 0.01 \\ & & \text { HIGH INPUT LIMIT : } 327.67\end{array}$

PAGE C 12 OF C 50 


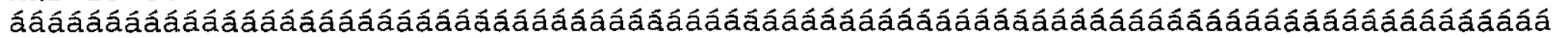

SCREEN \#020: DATA DISPLAY

SCREEN MEMORY USED: 203 BYTES

PREVIOUS MENU: $\quad 001$

NEXT SCREEN : 027

PREVIOUS SCREEN : UNLINKED

DATA POSITION \#1: 16 BIT UNSIGNED INTEGER DISPLAY

$\begin{array}{lll}\text { REGISTER : } & \mathrm{N} 60: 14 \\ \text { DECIMAL } & \text { RIGHT: } & 1 \\ \text { DECIMAL } & \text { LEFT: } & 2 \\ \text { NO SIGN } & \text { PLACE } & \\ \text { LEADING } & \text { ZEROS NOT } & \text { SHOWN }\end{array}$

\{́ëëëëëëëëëëëëëëëëë i

- Water Tank BDID in

-PLC Temp BS25 F o

- Compr Temp BD3 F 。

- Pump Temp DD4P F åëëëëëëëëëëëëëëëëëë $f$

DATA POSITION \#2: 16 BIT SIGNED INTEGER DISPLAY

$\begin{array}{llll}\text { REGISTER : } & \text { N60:27 } & \text { REGISTER LOW : } & -454 \\ \text { DECIMAL RIGHT : } & 0 & \text { REGISTER HIGH : } & 752 \\ \text { DECIMAL LEFT : } & 3 & \text { DISPLAY LOW : } & -454 \\ \text { SHOW SIGN } & & \text { DISPLAY HIGH : } & 752\end{array}$

LEADING ZEROS NOT SHOWN

REGISTER LOW: $\quad 3277$

REGISTER HIGH : $\quad 16384$

DISPLAY LOW: 0.0

DISPLAY HIGH: $\quad 62.0$

DATA POSITION \#3: 16 BIT SIGNED INTEGER DISPLAY

$\begin{array}{llll}\text { REGISTER : } & \text { N60:26 } & \text { REGISTER LOW: } & -454 \\ \text { DECIMAL RIGHT: } & 0 & \text { REGISTER HIGH: } & 752 \\ \text { DECIMAL LEFT : } & 3 & \text { DISPLAY LOW : } & -454 \\ \text { SHOW SIGN } & & \text { DISPLAY HIGH: } & 752 \\ \text { LEADING ZEROS NOT SHOWN } & & \end{array}$

DATA POSITION \#4: 16 BIT SIGNED INTEGER DISPLAY

$\begin{array}{llll}\text { REGISTER : } & \text { N60:24 } & \text { REGISTER LOW : } & -454 \\ \text { DECIMAL RIGHT: } & 0 & \text { REGISTER HIGH: } & 752 \\ \text { DECIMAL LEFT: } & 3 & \text { DISPLAY LOW : } & -454 \\ \text { SHOW SIGN } & & \text { DISPLAY HIGH: } & 752 \\ \text { LEADING ZEROS NOT SHOWN } & & \end{array}$

PAGE C 13 OF C 50 


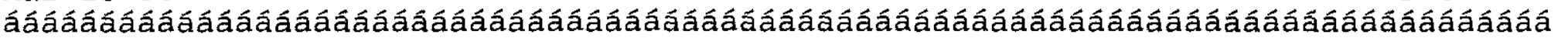

SCREEN \#021: DATA ENTRY

SCREEN MEMORY USED: 185 BYTES

PREVIOUS MENU: 017

NEXT SCREEN : 018

PREVIOUS SCREEN: UNLINKED

DATA POSITION \#1: 16 BIT UNSIGNED INTEGER DISPLAY

$\begin{array}{llll}\text { REGISTER : } & \text { N20:23 } & \text { REGISTER LOW: } & 0 \\ \text { DECIMAL RIGHT: } & 0 & \text { REGISTER HIGH : } & 16383 \\ \text { DECIMAL LEFT: } & 5 & \text { DISPLAY LOW : } & 0 \\ \text { NO SIGN PLACE } & & \text { DISPLAY HIGH: } & 100 \\ \text { LEADING ZEROS NOT SHOWN } & & \end{array}$

DATA POSITION \#2: 16 BIT UNSIGNED INTEGER DISPLAY

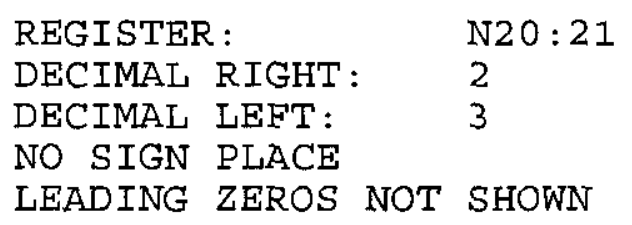

DATA POSITION \#3: 16 BIT UNSIGNED INTEGER ENTRY

$\begin{array}{llll}\text { REGISTER : } & \text { N20:21 } & \text { REGISTER LOW : } & 1 \\ \text { DECIMAL RIGHT: } & 2 & \text { REGISTER HIGH: } & 16383 \\ \text { DECIMAL LEFT: } & 3 & \text { DISPLAY LOW : } & 0.01 \\ \text { NO SIGN PLACE } & & \text { DISPLAY HIGH : } & 327.67 \\ \text { DEFAULT : } & \text { NONE } & \text { LOW INPUT LIMIT: } 0.01 \\ & & \text { HIGH INPUT LIMIT : } 327.67\end{array}$
REGISTER LOW:
1
REGISTER HIGH: $\quad 16383$
DISPLAY LOW: $\quad 0.01$
DISPLAY HIGH: $\quad 327.67$ DAR \{́ëëëëëëëëëëëëëëëëëë

${ }^{\circ}$ Derv 0.01 to $327.67^{\circ}$

${ }^{\circ} \mathrm{DOV}$ OUTPUT: $\mathrm{DD} \mathrm{BD} \div$

Derivative: DPD2B口。

- Enter: DDS3DD

åëëëëëëëëëëëëëëëëëëêf 


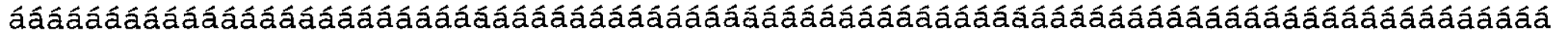

SCREEN \#022: DATA ENTRY

SCREEN MEMORY USED: 157 BYTES

PREVIOUS MENU: 010

NEXT SCREEN : 013

PREVIOUS SCREEN : UNLINKED

DATA POSITION \#1: BIT DISPLAY

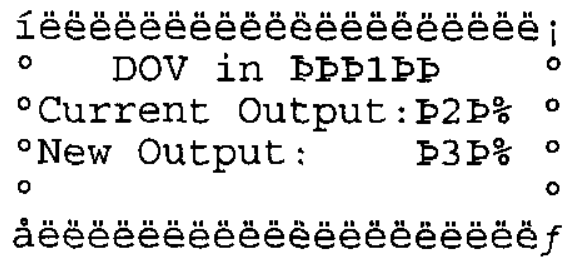

OFF BIT TEXT: Manual ON BIT TEXT: AUTO

DATA POSITION \#2: 16 BIT UNSIGNED INTEGER DISPLAY

$\begin{array}{llll}\text { REGISTER : } & \text { N20:23 } & \text { REGISTER LOW: } & 0 \\ \text { DECIMAL RIGHT: } & 0 & \text { REGISTER HIGH : } & 16383 \\ \text { DECIMAL LEFT: } & 3 & \text { DISPLAY LOW: } & 0 \\ \text { NO SIGN PLACE } & & \text { DISPLAY HIGH : } & 100\end{array}$

DATA POSITION \#3: 16 BIT UNSIGNED INTEGER ENTRY

$\begin{array}{llll}\text { REGISTER : } & \text { N20:23 } & \text { REGISTER LOW: } & 0 \\ \text { DECIMAL RIGHT: } & 0 & \text { REGISTER HIGH: } & 16384 \\ \text { DECIMAL LEFT: } & 3 & \text { DISPLAY LOW : } & 0 \\ \text { NO SIGN PLACE } & & \text { DISPLAY HIGH : } & 100 \\ \text { DEFAULT: } & \text { NONE } & \text { LOW INPUT LIMIT: } 0 \\ & & \text { HIGH INPUT LIMIT : } 100\end{array}$

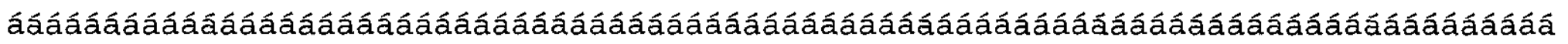

SCREEN \#023: DATA ENTRY

SCREEN MEMORY USED: 94 BYTES

PREVIOUS MENU: 001

NEXT SCREEN : 001

PREVIOUS SCREEN: UNLINKED

DATA POSITION \#1: BIT ENTRY

REGISTER: $\quad$ N2 $0: 17$

BIT NUMBER: $\quad 10$

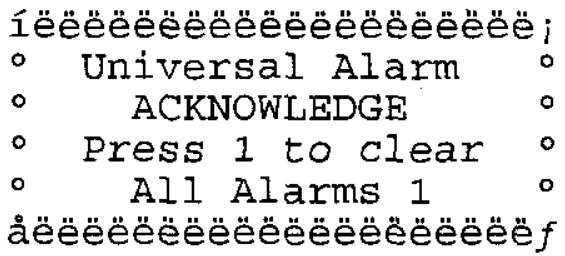

ENTRY FORMAT :

DEFAULT :
$1 \& 0$ KEYS NONE

PAGE C 15 OF C 50 


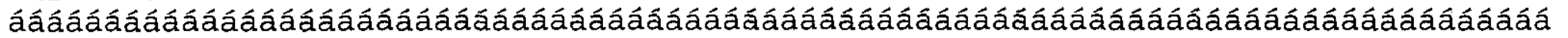

SCREEN \#024: DATA DISPLAY

SCREEN MEMORY USED: 194 BYTES

PREVIOUS MENU: 001

NEXT SCREEN : 025

PREVIOUS SCREEN : UNLINKED

DATA POSITION \#1: 16 BIT UNSIGNED INTEGER DISPLAY

REGISTER: $\quad$ N60:12

DECIMAL RIGHT: 0

DECIMAL LEFT: $\quad 5$

NO SIGN PLACE

LEADING ZEROS NOT SHOWN 1́ëëëëëëëëëëëëëëëëëë

- I: 2.0 BS1DD SGT

- I:2.1 DD2DD WFT

${ }^{\circ} \mathrm{I}: 2.2$ DS3ED CVT

○I: 2.3 DD4DD WTL

åëëëëëëëëëëëëëëëëëëëf

DATA POSITION \#2: 16 BIT UNSIGNED INTEGER DISPLAY

$\begin{array}{llll}\text { REGISTER : } & \text { N60:13 } & \text { REGISTER LOW : } & 0 \\ \text { DECIMAL RIGHT: } & 0 & \text { REGISTER HIGH : } & 32767 \\ \text { DECIMAL LEFT: } & 5 & \text { DISPLAY LOW : } & 0 \\ \text { NO SIGN PLACE } & & \text { DISPLAY HIGH : } & 32767\end{array}$

DATA POSITION \#3: 16 BIT UNSIGNED INTEGER DISPLAY

$\begin{array}{llll}\text { REGISTER : } & \text { N60:15 } & \text { REGISTER LOW : } & 0 \\ \text { DECIMAL RIGHT: } & 0 & \text { REGISTER HIGH : } & 32767 \\ \text { DECIMAL LEFT: } & 5 & \text { DISPLAY LOW : } & 0 \\ \text { NO SIGN PLACE } & & \text { DISPLAY HIGH : } & 32767\end{array}$

LEADING ZEROS NOT SHOWN

DATA POSITION \#4: 16 BIT UNSIGNED INTEGER DISPLAY

$\begin{array}{llll}\text { REGISTER : } & \text { N60:14 } & \text { REGISTER LOW: } & 0 \\ \text { DECIMAL RIGHT: } & 0 & \text { REGISTER HIGH: } & 32767 \\ \text { DECIMAL LEFT: } & 5 & \text { DISPLAY LOW: } & 0 \\ \text { NO SIGN PLACE } & & \text { DISPLAY HIGH : } & 32767 \\ \text { LEADING ZEROS NOT SHOWN } & & \end{array}$

PAGE C 16 OF C 50 


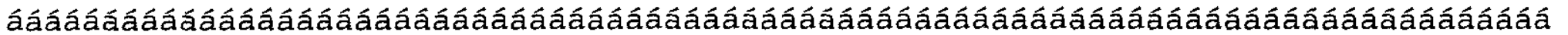

SCREEN \#025: DATA DISPLAY

SCREEN MEMORY USED: 210 BYTES

PREVIOUS MENU: UNLINKED

NEXT SCREEN: 026

PREVIOUS SCREEN : UNLINKED

DATA POSITION \#1: 16 BIT SIGNED INTEGER DISPLAY

$\begin{array}{llll}\text { REGISTER : } & \text { N60:26 } & \text { REGISTER LOW : } & -454 \\ \text { DECIMAL RIGHT : } & 0 & \text { REGISTER HIGH : } & 752 \\ \text { DECIMAL LEFT : } & 3 & \text { DISPLAY LOW : } & -454 \\ \text { SHOW SIGN } & & \text { DISPLAY HIGH : } & 752\end{array}$

\{́ëëëëëëëëëëëëëëëëëë

- COMPRS temp DEID F。

- PUMP temp DE2D F 。

- JMPR temp DD3D F 。

${ }^{\circ} \mathrm{PLC}$ CAB temp DS4D F 0 åëëëëëëëëëëëëëëëëëëf

LEADING ZEROS NOT SHOWN

DATA POSITION \#2: 16 BIT SIGNED INTEGER DISPLAY

$\begin{array}{llll}\text { REGISTER: } & \text { N60:24 } & \text { REGISTER LOW : } & -454 \\ \text { DECIMAL RIGHT: } & 0 & \text { REGISTER HIGH: } & 752 \\ \text { DECIMAI LEFT : } & 3 & \text { DISPLAY LOW : } & -454 \\ \text { SHOW SIGN } & & \text { DISPLAY HIGH : } & 752\end{array}$

LEADING ZEROS NOT SHOWN

DATA POSITION \#3: 16 BIT SIGNED INTEGER DISPLAY

$\begin{array}{llll}\text { REGISTER : } & \text { N60:25 } & \text { REGISTER LOW : } & -454 \\ \text { DECIMAL RIGHT : } & 0 & \text { REGISTER HIGH : } & 752 \\ \text { DECIMAL LEFT : } & 3 & \text { DISPLAY LOW : } & -454 \\ \text { SHOW SIGN } & & \text { DISPLAY HIGH : } & 752\end{array}$

DATA POSITION \#4: 16 BIT SIGNED INTEGER DISPLAY

$\begin{array}{llll}\text { REGISTER : } & \text { N60:27 } & \text { REGISTER LOW: } & -454 \\ \text { DECIMAL RIGHT : } & 0 & \text { REGISTER HIGH: } & 752 \\ \text { DECIMAL LEFT: } & 3 & \text { DISPLAY LOW : } & -454 \\ \text { SHOW SIGN } & & \text { DISPLAY HIGH: } & 752 \\ \text { LEADING ZEROS NOT SHOWN } & & \end{array}$

LEADING ZEROS NOT SHOWN

PAGE C 17 OF C 50 


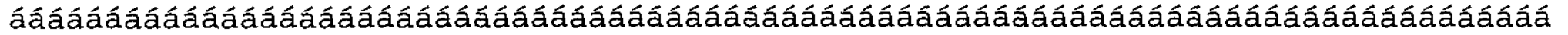

SCREEN \#026: DATA DISPLAY

1́ëëëëëëëëëëëëëëëëëë i

SCREEN MEMORY USED: 239 BYTES

PREVIOUS MENU: UNLINKED

NEXT SCREEN : 001

PREVIOUS SCREEN : UNLINKED

- PMP DISC D1P psi。

-PMP FLOW \$D2D gpm ०

-TOTAL DE3DDSL gal。

- FGM DS5D \%

ฉ̊ëëëëëëëëëëëëëëëëëë $f$

DATA POSITION \#1: 16 BIT UNSIGNED INTEGER DISPIAY

$\begin{array}{llll}\text { REGISTER : } & \text { N60:6 } & \text { REGISTER LOW: } & 3277 \\ \text { DECIMAL RIGHT: } & 0 & \text { REGISTER HIGH : } & 16383 \\ \text { DECIMAL LEFT: } & 3 & \text { DISPLAY LOW : } & 0 \\ \text { NO SIGN PLACE } & & \text { DISPLAY HIGH : } & 300\end{array}$

LEADING ZEROS NOT SHOWN

DATA POSITION \#2: 16 BIT UNSIGNED INTEGER DISPLAY

$\begin{array}{llll}\text { REGISTER: } & \text { N60:15 } & \text { REGISTER LOW : } & 3277 \\ \text { DECIMAL RIGHT: } & 2 & \text { REGISTER HIGH : } & 16383 \\ \text { DECIMAL LEFT : } & 1 & \text { DISPLAY LOW : } & 0.00 \\ \text { NO SIGN PLACE } & & \text { DISPLAY HIGH : } & 8.00\end{array}$

LEADING ZEROS NOT SHOWN

DATA POSITION \#3: 16 BIT UNSIGNED INTEGER DISPLAY

$\begin{array}{llll}\text { REGISTER : } & \text { C5:2.2 } & \text { REGISTER LOW : } & 0 \\ \text { DECIMAL RIGHT: } & 0 & \text { REGISTER HIGH : } & 9999 \\ \text { DECIMAL LEFT: } & 4 & \text { DISPLAY LOW : } & 0 \\ \text { NO SIGN PLACE } & & \text { DISPLAY HIGH : } & 9999 \\ \text { LEADING ZEROS NOT SHOWN } & & \end{array}$

DATA POSITION \#4: 16 BIT UNSIGNED INTEGER DISPLAY

$\begin{array}{llll}\text { REGISTER : } & \text { C5:1.2 } & \text { REGISTER LOW : } & 0 \\ \text { DECIMAL RIGHT: } & 0 & \text { REGISTER HIGH : } & 9999 \\ \text { DECIMAL LEFT: } & 4 & \text { DISPLAY LOW : } & 0 \\ \text { NO SIGN PLACE } & & \text { DISPLAY HIGH : } & 9999\end{array}$

DATA POSITION \#5: 16 BIT UNSIGNED INTEGER DISPLAY

$\begin{array}{llll}\text { REGISTER : } & \text { N60:2 } & \text { REGISTER LOW: } & 3277 \\ \text { DECIMAL RIGHT: } & 1 & \text { REGISTER HIGH: } & 16383 \\ \text { DECIMAL LEFT: } & 2 & \text { DISPLAY LOW: } & 0.0 \\ \text { NO SIGN PLACE } & & \text { DISPLAY HIGH: } & 30.0\end{array}$

LEADING ZEROS NOT SHOWN

PAGE C 18 OF C 50 


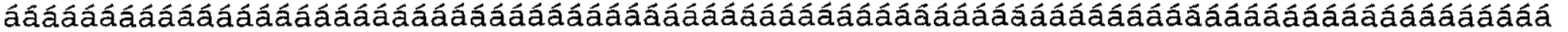

SCREEN \#027: DATA DISPLAY

SCREEN MEMORY USED: 241 BYTES

PREVIOUS MENU: $\quad$ UNLINKED

NEXT SCREEN : 028

PREVIOUS SCREEN: UNLINKED

DATA POSITION \#1: 16 BIT SIGNED INTEGER DISPLAY 1́ëëëëëëëëëëëëëëëëëe - JMPER TEMP DDID F。

- PUMP DISC D2D psi० -PUMP FLOW DS3D gpm -TOTAL DD4DDL5D gal。 åëëëëëëëëëëëëëëëëëë f

$\begin{array}{llll}\text { REGISTER : } & \text { N60:25 } & \text { REGISTER LOW : } & -454 \\ \text { DECIMAL RIGHT : } & 0 & \text { REGISTER HIGH : } & 752 \\ \text { DECIMAL LEFT : } & 3 & \text { DISPLAY LOW : } & -454 \\ \text { SHOW SIGN } & & \text { DISPLAY HIGH : } & 752\end{array}$

LEADING ZEROS NOT SHOWN

DATA POSITION \#2: 16 BIT UNSIGNED INTEGER DISPLAY

$\begin{array}{llll}\text { REGISTER : } & \text { N60:6 } & \text { REGISTER LOW: } & 3277 \\ \text { DECIMAL RIGHT: } & 0 & \text { REGISTER HIGH : } & 16383 \\ \text { DECIMAL LEFT: } & 3 & \text { DISPLAY LOW : } & 0 \\ \text { NO SIGN PLACE } & & \text { DISPLAY HIGH: } & 300\end{array}$

LEADING ZEROS NOT SHOWN

DATA POSITION \#3: 16 BIT UNSIGNED INTEGER DISPLAY

$\begin{array}{llll}\text { REGISTER: } & \text { N60:15 } & \text { REGISTER LOW: } & 3277 \\ \text { DECIMAL RIGHT: } & 2 & \text { REGISTER HIGH: } & 16383 \\ \text { DECIMAL LEFT: } & 1 & \text { DISPLAY LOW: } & 0.00 \\ \text { NO SIGN PLACE } & & \text { DISPLAY HIGH: } & 8.00\end{array}$

DATA POSITION \#4: 16 BIT UNSIGNED INTEGER DISPLAY

$\begin{array}{llll}\text { REGISTER : } & \text { C5:2.2 } & \text { REGISTER LOW : } & 0 \\ \text { DECIMAL RIGHT: } & 0 & \text { REGISTER HIGH : } & 9999 \\ \text { DECIMAL LEFT: } & 4 & \text { DISPLAY LOW: } & 0 \\ \text { NO SIGN PLACE } & & \text { DISPLAY HIGH : } & 9999\end{array}$

LEADING ZEROS NOT SHOWN

DATA POSITION \#5: 16 BIT UNSIGNED INTEGER DISPLAY

$\begin{array}{llll}\text { REGISTER : } & \text { C5:1.2 } & \text { REGISTER LOW : } & 0 \\ \text { DECIMAL RIGHT: } & 0 & \text { REGISTER HIGH : } & 9999 \\ \text { DECIMAL LEFT: } & 4 & \text { DISPLAY LOW : } & 0 \\ \text { NO SIGN PLACE } & & \text { DISPLAY HIGH : } & 9999\end{array}$

LEADING ZEROS NOT SHOWN

PAGE C 19 OF C 50 


\section{RPP-5492 REVISION 0, APPENDIX C}

Mar 1500

FILE: U109NEW1. CFG

PAGE 17

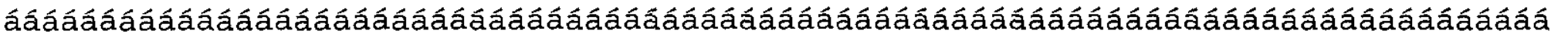

SCREEN \#028: DATA DISPLAY

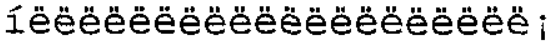

SCREEN MEMORY USED: 172 BYTES

PREVIOUS MENU: UNLINKED

$\begin{array}{ll}\text { NEXT SCREEN : } & 029 \\ \text { PREVIOUS SCREEN : } & \text { UNLINKED }\end{array}$

-PIT CGM DD1DD\%。

${ }^{\circ} \mathrm{DOME}$ FGM $\mathrm{DS} 2 \mathrm{5} \div$

ODIL RATIO D3\%

-TIME $08: 50 \circ$

åëëëëëëëëëëëëëëëëëëf

DATA POSITION \#1: 16 BIT UNSIGNED INTEGER DISPLAY

$\begin{array}{llll}\text { REGISTER : } & \text { N60:2 } & \text { REGISTER LOW: } & 3277 \\ \text { DECIMAL RIGHT: } & 1 & \text { REGISTER HIGH: } & 16383 \\ \text { DECIMAL LEFT: } & 3 & \text { DISPLAY LOW: } & 0.0 \\ \text { NO SIGN PLACE } & & \text { DISPLAY HIGH: } & 100.0\end{array}$

DATA POSITION \#2: 16 BIT UNSIGNED INTEGER DISPLAY

$\begin{array}{llll}\text { REGISTER : } & \text { N60:3 } & \text { REGISTER LOW: } & 3277 \\ \text { DECIMAL RIGHT: } & 1 & \text { REGISTER HIGH : } & 16383 \\ \text { DECIMAL LEFT: } & 3 & \text { DISPLAY LOW: } & 0.0 \\ \text { NO SIGN PLACE } & & \text { DISPLAY HIGH: } & 100.0\end{array}$

DATA POSITION \#3: 16 BIT SIGNED INTEGER DISPLAY

$\begin{array}{llll}\text { REGISTER : } & \text { N30:3 } & \text { REGISTER LOW : } & 0 \\ \text { DECIMAL RIGHT : } & 0 & \text { REGISTER HIGH : } & 50 \\ \text { DECIMAL LEFT : } & 2 & \text { DISPLAY LOW : } & 0 \\ \text { NO SIGN PLACE } & & \text { DISPLAY HIGH : } & 50\end{array}$

SHOW LEADING ZEROS

PAGE C 20 OF C 50 


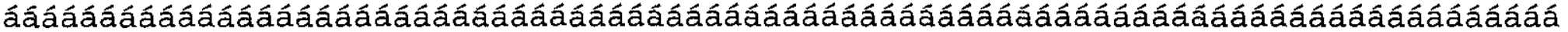

SCREEN \#029: DATA DISPLAY

SCREEN MEMORY USED: 210 BYTES

PREVIOUS MENU: UNLINKED

NEXT SCREEN : $\quad 037$

PREVIOUS SCREEN: 028

DATA POSITION \#1: 16 BIT UNSIGNED INTEGER DISPLAY \{́ëëëëëëëëëëëëëëëëëë; - RECR FL PR DS1D PSI。 ${ }^{\circ}$ PS2 FL PR DE25 PSI $\circ$ $X$ XFR PRESS B3P PSI $\circ$ -MIN DIL DD4P GPM ० åëëëëëëëëëëëëëëëëëë $f$

$\begin{array}{llll}\text { REGISTER : } & \text { N60:16 } & \text { REGISTER LOW: } & 3277 \\ \text { DECIMAL RIGHT: } & 1 & \text { REGISTER HIGH: } & 16383 \\ \text { DECIMAL LEFT: } & 2 & \text { DISPLAY LOW: } & 0.0 \\ \text { NO SIGN PLACE } & & \text { DISPLAY HIGH: } & 30.0\end{array}$

DATA POSITION \#2: 16 BIT UNSIGNED INTEGER DISPLAY

$\begin{array}{llll}\text { REGISTER : } & \text { N60:17 } & \text { REGISTER LOW: } & 3277 \\ \text { DECIMAL RIGHT: } & 1 & \text { REGISTER HIGH : } & 16383 \\ \text { DECIMAL LEFT: } & 2 & \text { DISPLAY LOW : } & 0.0 \\ \text { NO SIGN PLACE } & & \text { DISPLAY HIGH : } & 30.0\end{array}$

DATA POSITION \#3: 16 BIT UNSIGNED INTEGER DISPLAY

$\begin{array}{llll}\text { REGISTER : } & \text { N60:18 } & \text { REGISTER LOW : } & 3277 \\ \text { DECIMAL RIGHT: } & 0 & \text { REGISTER HIGH: } & 16383 \\ \text { DECIMAL LEFT: } & 3 & \text { DISPLAY LOW: } & 0 \\ \text { NO SIGN PLACE } & & \text { DISPLAY HIGH : } & 300\end{array}$

DATA POSITION \#4: 16 BIT UNSIGNED INTEGER DISPLAY

$\begin{array}{llll}\text { REGISTER : } & \text { N30:6 } & \text { REGISTER LOW : } & 6240 \\ \text { DECIMAL RIGHT: } & 2 & \text { REGISTER HIGH : } & 31208 \\ \text { DECIMAL LEFT: } & 1 & \text { DISPLAY LOW : } & 0.00 \\ \text { NO SIGN PLACE } & & \text { DISPLAY HIGH : } & 2.50\end{array}$

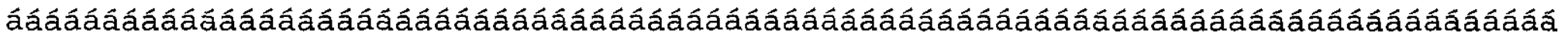

SCREEN \#030: SUB-MENU

SCREEN MEMORY USED: 64 BYTES

PREVIOUS MENU: 001

Item/Key \# 1 links to screen 031

Item/Key \#2 links to screen 032

Item/Key \# 3 links to screen 033

Item/Key \# 4 is unlinked

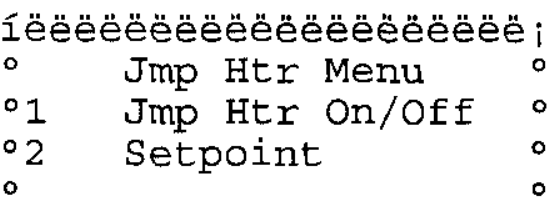

åëëëëëëëëëëëëëëëëëëëf

Item/Key \# 5 is unlinked

Item/Key \# 6 is unlinked

Item/Key \# 7 is unlinked

Item/Key \# 8 is unlinked

PAGE C 21 OF C 50 


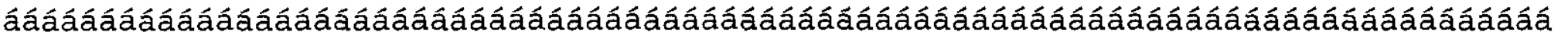

SCREEN \#031: DATA ENTRY

SCREEN MEMORY USED: 111 BYTES

PREVIOUS MENU: $\quad 030$

NEXT SCREEN : 001

PREVIOUS SCREEN : UNLINKED

DATA POSITION \#1: BIT DISPLAY \{́ëëëëëëëëëëëëëëëëëë i

- U-109 Jmp Htr o

- Jmp Her is DID

- Enter 1 for On

- 0 for OFF: 2 o

åe̊ëëëëëëëëëëëëëëëëë $f$
OFF BIT TEXT: OFF

ON BIT TEXT: ON

DATA POSITION \#2: BIT ENTRY
REGISTER :
N2 $0: 17$
BIT NUMBER :
4
ENTRY FORMAT:
DEFAULT :
$1 \& 0$ KEYS
NONE

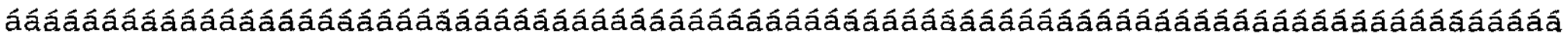

SCREEN \#032: DATA ENTRY

SCREEN MEMORY USED: 167 BYTES

PREVIOUS MENU: $\quad 030$

NEXT SCREEN : 001

PREVIOUS SCREEN : UNLINKED

DATA POSITION \#1: 16 BIT SIGNED INTEGER DISPLAY 1́ëëëëëëëëëëëëëëëëëë;

- JumperTemp: DDDIDSF'

$\circ$

-Old Setpoint:

- New setpoint: $\mathrm{DS} 3 \mathrm{DSF}{ }^{\circ}$

åëëëëëëëëëëëëëëëëëë $f$
REGISTER :
N60 $: 25$
DECIMAL RIGHT: 0
DECIMAL LEFT: 5
REGISTER LOW: $\quad-454$
REGISTER HIGH : 752
SHOW SIGN
DISPLAY LOW:
$-454$
DISPLAY HIGH : $\quad 752$

LEADING ZEROS NOT SHOWN

DATA POSITION \#2: 16 BIT SIGNED INTEGER DISPLAY

$\begin{array}{llll}\text { REGISTER : } & \text { N31:4 } & \text { REGISTER LOW : } & -454 \\ \text { DECIMAL RIGHT : } & 0 & \text { REGISTER HIGH : } & 752 \\ \text { DECIMAL LEFT: } & 5 & \text { DISPLAY LOW : } & -454 \\ \text { SHOW SIGN } & & \text { DISPLAY HIGH : } & 752 \\ \text { LEADING ZEROS NOT SHOWN } & & \end{array}$

DATA POSITION \#3: 16 BIT SIGNED INTEGER ENTRY

$\begin{array}{llll}\text { REGISTER : } & \text { N31:4 } & \text { REGISTER LOW : } & -454 \\ \text { DECIMAL RIGHT: } & 0 & \text { REGISTER HIGH : } & 752 \\ \text { DECIMAL LEFT : } & 5 & \text { DISPLAY LOW : } & -454 \\ \text { SHOW SIGN } & & \text { DISPLAY HIGH : } & 752 \\ \text { DEFAULT : } & \text { NONE } & \text { LOW INPUT LIMIT: } & 50 \\ & & \text { HIGH INPUT LIMIT : } 200\end{array}$

PAGE C 22 OF C 50 


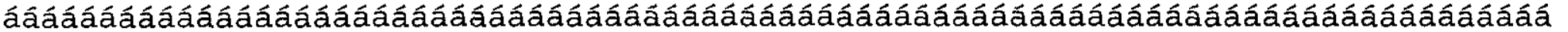

SCREEN \#033: DATA DISPLAY

SCREEN MEMORY USED: 211 BYTES

PREVIOUS MENU: $\quad 030$

NEXT SCREEN: 001

PREVIOUS SCREEN: UNLINKED

DATA POSITION \#1: 16 BIT SIGNED INTEGER DISPLAY 1́ëëëëëëëëëëëëëëëëë

-Shutoff T/C: DDDIDD F०

'Shutoff S.P: DDS2DD F०

- Control T/C: DES3ED Fo

- Control S.P: DSL4DS Fo åëëëëëëëëëëëëëëëëëëë $f$

$\begin{array}{llll}\text { REGISTER : } & \text { N31:2 } & \text { REGISTER LOW : } & -454 \\ \text { DECIMAL RIGHT: } & 0 & \text { REGISTER HIGH: } & 752 \\ \text { DECIMAL LEFT : } & 5 & \text { DISPLAY LOW : } & -454 \\ \text { SHOW SIGN } & & \text { DISPLAY HIGH : } & 752\end{array}$

LEADING ZEROS NOT SHOWN

DATA POSITION \#2: 16 BIT SIGNED INTEGER DISPLAY

$\begin{array}{llll}\text { REGISTER : } & \text { N31:5 } & \text { REGISTER LOW : } & -454 \\ \text { DECIMAL RIGHT : } & 0 & \text { REGISTER HIGH : } & 752 \\ \text { DECIMAL LEFT : } & 5 & \text { DISPLAY LOW : } & -454 \\ \text { SHOW SIGN } & & \text { DISPLAY HIGH : } & 752\end{array}$

DATA POSITION \#3: 16 BIT SIGNED INTEGER DISPLAY

$\begin{array}{llll}\text { REGISTER: } & \text { N31:1 } & \text { REGISTER LOW: } & -454 \\ \text { DECIMAL RIGHT: } & 0 & \text { REGISTER HIGH: } & 752 \\ \text { DECIMAL LEFT: } & 5 & \text { DISPLAY LOW : } & -454 \\ \text { SHOW SIGN } & & \text { DISPLAY HIGH : } & 752 \\ \text { LEADING ZEROS NOT SHOWN } & & \end{array}$

DATA POSITION \#4: 16 BIT SIGNED INTEGER DISPLAY

$\begin{array}{llll}\text { REGISTER : } & \text { N31:4 } & \text { REGISTER LOW : } & -454 \\ \text { DECIMAL RIGHT : } & 0 & \text { REGISTER HIGH : } & 752 \\ \text { DECIMAL LEFT : } & 5 & \text { DISPLAY LOW : } & -454 \\ \text { SHOW SIGN } & & \text { DISPLAY HIGH : } & 752 \\ \text { LEADING ZEROS NOT SHOWN } & & \end{array}$

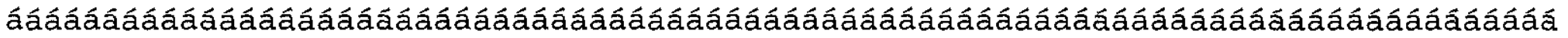

SCREEN \#034: SUB-MENU

1́ëëëëëëëëëëëëëëëëëë;

SCREEN MEMORY USED: 68 BYTES

PREVIOUS MENU: 001

- DILUTION MENU

- 1. DILUTION RATIO ०

- 2. MIN DIL FLOW

åëëëëëëëëëëëëëëëëëëëf

Item/Key \# 1 links to screen 035

Item/Key \# 5 is unlinked

Item/Key \# 2 links to screen 036

Item/Key \# 6 is unlinked

Item/Key \# 3 is unlinked

Item/Key \# 4 is unlinked

Item/Key \# 7 is unlinked

Item/Key \# 8 is unlinked

PAGE C 23 OF C 50 


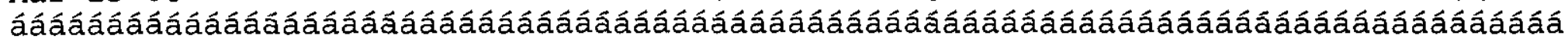

SCREEN \#035: DATA ENTRY

SCREEN MEMORY USED: 133 BYTES

PREVIOUS MENU: 034

NEXT SCREEN: 001

PREVIOUS SCREEN: UNLINKED

DATA POSITION \#1: 16 BIT SIGNED INTEGER DISPLAY \{َëëëëëëëëëëëëëëëëëë i

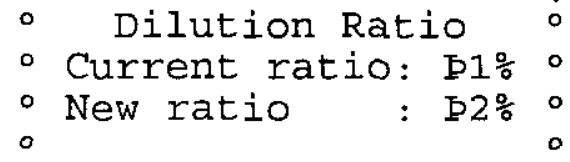

åëëëëëëëëëëëëëëëëëëf

$\begin{array}{llll}\text { REGISTER : } & \text { N30:3 } & \text { REGISTER LOW : } & 0 \\ \text { DECIMAL RIGHT: } & 0 & \text { REGISTER HIGH : } & 50 \\ \text { DECIMAL LEFT: } & 2 & \text { DISPLAY LOW: } & 0 \\ \text { NO SIGN PLACE } & & \text { DISPLAY HIGH : } & 50\end{array}$

DATA POSITION \#2: 16 BIT SIGNED INTEGER ENTRY

$\begin{array}{llll}\text { REGISTER : } & \text { N30:3 } & \text { REGISTER LOW : } & 0 \\ \text { DECIMAL RIGHT: } & 0 & \text { REGISTER HIGH : } & 50 \\ \text { DECIMAL LEFT: } & 2 & \text { DISPLAY LOW : } & 0 \\ \text { NO SIGN PLACE } & & \text { DISPLAY HIGH : } & 50 \\ \text { DEFAULT: } & \text { NONE } & \text { LOW INPUT LIMIT: } & 0 \\ & & \text { HIGH INPUT LIMIT: } 50\end{array}$

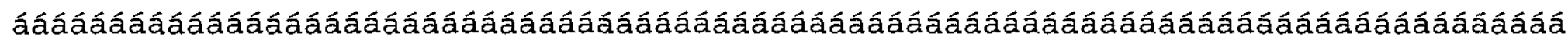

SCREEN \#036: DATA ENTRY

SCREEN MEMORY USED: 131 BYTES

PREVIOUS MENU: 034

NEXT SCREEN : 001

PREVIOUS SCREEN :

UNLINKED

DATA POSITION \#1: 16 BIT UNSIGNED INTEGER DISPLAY \{̇ëëëëëëëëëëëëëëëëëë

- MIN DIL FLOW

- MIN FLOW: DPID GPM 。

- NEW FLOW: DE2D GPM ०

åëëëëëëëëëëëëëëëëëëëf

$\begin{array}{llll}\text { REGISTER : } & \text { N30:6 } & \text { REGISTER LOW: } & 6240 \\ \text { DECIMAL RIGHT: } & 2 & \text { REGISTER HIGH : } & 31208 \\ \text { DECIMAL LEFT: } & 1 & \text { DISPLAY LOW : } & 0.00 \\ \text { NO SIGN PLACE } & & \text { DISPLAY HIGH : } & 2.50\end{array}$

LEADING ZEROS NOT SHOWN

DATA POSITION \#2: 16 BIT UNSIGNED INTEGER ENTRY

$\begin{array}{ll}\text { REGISTER: } & \text { N30:6 } \\ \text { DECIMAL RIGHT: } & 2 \\ \text { DECIMAL LEFT: } & 1 \\ \text { NO SIGN PLACE } & \\ \text { DEFAULT: } & 0.00\end{array}$

$\begin{array}{ll}\text { REGISTER LOW: } & 6240 \\ \text { REGISTER HIGH: } & 31208 \\ \text { DISPLAY LOW: } & 0.00 \\ \text { DISPLAY HIGH: } & 2.50 \\ \text { LOW INPUT LIMIT: } & 0.00 \\ \text { HIGH INPUT LIMIT: } & 2.50\end{array}$

PAGE C 24 OFC 50 


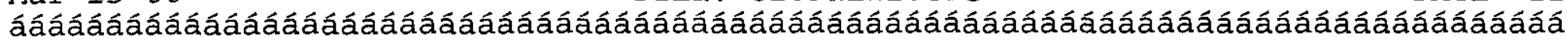

SCREEN \#037: DATA DISPLAY

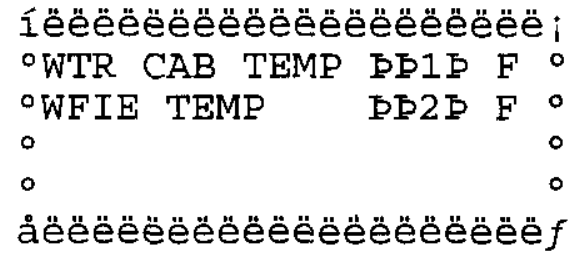

PREVIOUS MENU: UNLINKED

UNLINKED

029

PREVIOUS SCREEN :

DATA POSITION \#1: 16 BIT SIGNED INTEGER DISPLAY

$\begin{array}{llll}\text { REGISTER : } & \text { N60:32 } & \text { REGISTER LOW : } & -454 \\ \text { DECIMAL RIGHT: } & 0 & \text { REGISTER HIGH : } & 752 \\ \text { DECIMAL LEFT : } & 3 & \text { DISPLAY LOW: } & -454 \\ \text { SHOW SIGN } & & \text { DISPLAY HIGH: } & 752 \\ \text { LEADING ZEROS NOT SHOWN } & & \end{array}$

DATA POSITION \#2: 16 BIT SIGNED INTEGER DISPLAY

$\begin{array}{llll}\text { REGISTER : } & \text { N60:33 } & \text { REGISTER LOW : } & -454 \\ \text { DECIMAL RIGHT: } & 0 & \text { REGISTER HIGH : } & 752 \\ \text { DECIMAL LEFT : } & 3 & \text { DISPLAY LOW : } & -454 \\ \text { SHOW SIGN } & & \text { DISPLAY HIGH: } & 752 \\ \text { LEADING ZEROS NOT SHOWN } & & \end{array}$

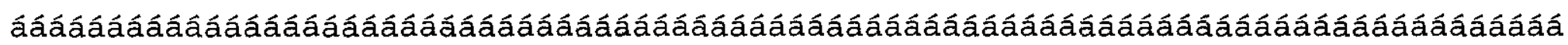

SCREEN \#040: SUB-MENU

1́ëëëëëëëëëëëëëëëëëë;

SCREEN MEMORY USED: 88 BYTES

PREVIOUS MENU: 001

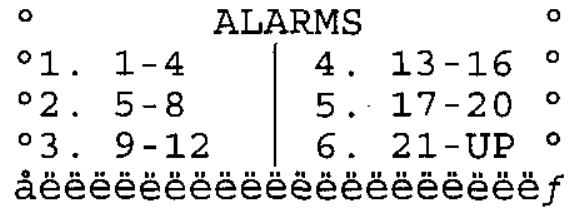

$\begin{array}{lllll}\text { Item/Key \# } & 1 & \text { links to screen } 041 \\ \text { Item/Key \# } & 2 & \text { links to screen } 042 \\ \text { Item/Key \# } & 3 & \text { links to screen } 043 \\ \text { Item/Key \# } 4 & \text { links to screen } 044\end{array}$

Item/Key \# 5 links to screen 045

Item/Key \# 6 links to screen 046

Item/Key \# 7 is unlinked

Item/Key \# 8 is unlinked 


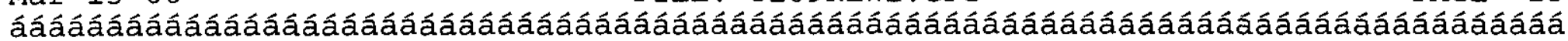

SCREEN \#041: DATA DISPLAY

SCREEN MEMORY USED: 181 BYTES

PREVIOUS MENU: NEXT SCREEN :

040 PREVIOUS SCREEN :

DATA POSITION \#1:

042 UNLINKED

REGISTER :

BIT NUMBER:

TEXT LENGTH:

DATA POSITION \#2:

REGISTER :

BIT NUMBER:

TEXT LENGTH:

DATA POSITION \#3:

REGISTER :

BIT NUMBER:

TEXT LENGTH :

DATA POSITION \#4:

REGISTER :

BIT NUMBER :

TEXT LENGTH :
$\mathrm{N} 20: 24$

1

4

BIT DISPLAY

$\mathrm{N} 20: 24$

2

4

BIT DISPLAY

$\mathrm{N} 20: 24$

3

4

BIT DISPLAY

$\mathrm{N} 20: 24$

4

7 \{́ëëëëëëëëëëëëëëëëëë

- XFR Pressure BDID

- XFR Pressure DL25。

- Flush Press ந\$3D。

- Pump DDBLDE ०

åęëëëëëëëëëëëëëëëëëëf

OFF BIT TEXT: norm

ON BIT TEXT: LOW

OFF BIT TEXT: norm

ON BIT TEXT: HIGH

OFF BIT TEXT: norm ON BIT TEXT: HIGH

OFF BIT TEXT:

norm

ON BIT TEXT:

TROUBLE

PAGE C 26 OF C 50 


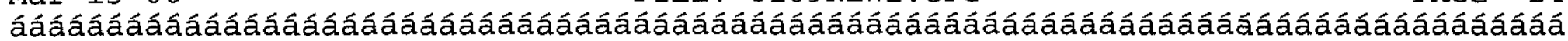

SCREEN \#042: DATA DISPLAY

SCREEN MEMORY USED: 191 BYTES

PREVIOUS MENU:

NEXT SCREEN :

040

PREVIOUS SCREEN :

DATA POSITION \#1:

043

UNLINKED

REGISTER :

BIT NUMBER:

TEXT LENGTH:

DATA POSITION \#2:

REGISTER :

BIT NUMBER:

TEXT LENGTH:

DATA POSITION \#3:

REGISTER :

BIT NUMBER:

TEXT LENGTH :

DATA POSITION \#4:

REGISTER :

BIT NUMBER: TEXT LENGTH :
8

7
N2 $0: 24$

5

11

\section{BIT DISPLAY}

$\mathrm{N} 20: 24$

6

4

OFF BIT TEXT:

ON BIT TEXT:

OFF BIT TEXT:

ON BIT TEXT:

OFF BIT TEXT:

ON BIT TEXT:

7

7

BIT DISPLAY

$\mathrm{N} 20: 24$

OFF BIT TEXT:

norm

ON BIT TEXT: norm

NON-PROCESS 


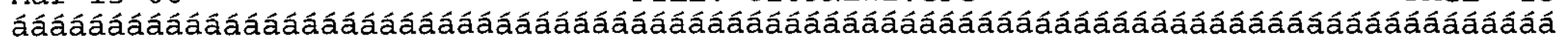

SCREEN \#043: DATA DISPLAY

SCREEN MEMORY USED: 182 BYTES

RREVIOUS MENU: $\quad 040$

NEXT SCREEN: $\quad 044$

PREVIOUS SCREEN : UNLINKED

DATA POSITION \#1: BIT DISPLAY

$\begin{array}{ll}\text { REGISTER: } & \text { N20:24 } \\ \text { BIT NUMBER : } & 9 \\ \text { TEXT LENGTH : } & 4\end{array}$

$\begin{array}{lr}\text { OFF BIT TEXT: } & \text { norm } \\ \text { ON BIT TEXT: } & \text { LOW }\end{array}$

\{́ëëëëëëëëëëëëëëëëëë

- PIC Water DD15。

- PLC Temp D\$25。

- CMPRSR Temp DS3E。

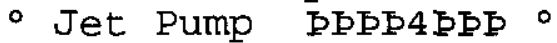
åëëëëëëëëëëëëëëëëëëë $f$

OFF BIT TEXT: norm ON BIT TEXT: HIGH

10

4

BIT NUMBER:

DATA POSITION \#3: BIT DISPLAY

REGISTER :

BIT NUMBER:

TEXT LENGTH:

$\mathrm{N} 20: 24$

11

4

OFF BIT TEXT: norm

ON BIT TEXT: HIGH

OFF BIT TEXT:

ON BIT TEXT:

norm

BIT NUMBER :

TEXT LENGTH:
N20:24

12

8

PAGE C 28 OF C 50 


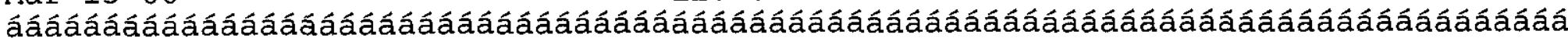

SCREEN \#044: DATA DISPLAY

SCREEN MEMORY USED: 181 BYTES

PREVIOUS MENU: $\quad 040$

NEX'T SCREEN :

PREVIOUS SCREEN :

DATA POSITION \#1:

REGISTER :

BIT NUMBER :

TEXT LENGTH :

045

UNLINKED

BIT DISPLAY

$\mathrm{N} 20: 24$

13

4

DATA POSITION \#2: BIT DISPLAY

$\begin{array}{ll}\text { REGISTER : } & \text { N20:24 } \\ \text { BIT NUMBER : } & 14 \\ \text { TEXT LENGTH : } & 4\end{array}$

DATA POSITION \#3: BIT DISPLAY

REGISTER :

BIT NUMBER:

TEXT LENGTH:

DATA POSITION \#4:

REGISTER :

BIT NUMBER:

TEXT LENGTH :
$\mathrm{N} 20: 24$

15

7

BIT DISPLAY

$\mathrm{N} 20: 25$

0

5 \{́ëëëëëëëëëëëëëëëëëë i

- SGT Level

DE150

- REPT SIGNAL DE2D 0

- Network DБВЗБББ

- JFPT SIGNAL DB4DE。

åëëëëëëëëëëëëëëëëëëf

OFF BIT TEXT:

ON BIT TEXT:

norm

LOW

OFF BIT TEXT: norm

ON BIT TEXT: LOST

OFF BIT TEXT:

norm

ON BIT TEXT:

FAILURE

OFF BIT TEXT:

ON BIT TEXT:

norm

LOST

PAGE C 29 OF C 50 


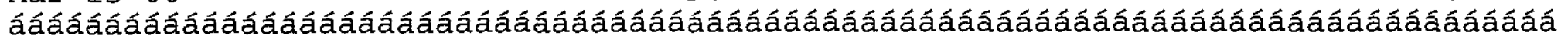

SCREEN \#045: DATA DISPLAY

SCREEN MEMORY USED: 182 BYTES

PREVIOUS MENU: NEXT SCREEN : PREVIOUS SCREEN :

DATA POSITION \#1:

REGISTER :

BIT NUMBER :

TEXT LENGTH:

DATA POSITION \#2:

REGISTER :

BIT NUMBER :

TEXT LENGTH:

DATA POSITION \#3:

REGISTER :

BIT NUMBER :

TEXT LENGTH :

DATA POSITION \#4:

REGISTER :

BIT NUMBER:

TEXT LENGTH :
040

046

UNLINKED

BIT DISPLAY

$\mathrm{N} 20: 25$

1

5

BIT DISPLAY

$\mathrm{N} 20: 25$

2

5

BIT DISPLAY

$\mathrm{N} 20: 25$

3

5

BIT DISPLAY

$\mathrm{N} 20: 25$

4

5 エૃëëëëëëëëëëëëëëëëëë i

- U Farm leak DP1DP 。

- Lk 1 COB U29 Dइ2Б5

- Leak $1 \mathrm{ck}$ БDЗББ。

- U Flush pos BD4DD åęëëëëëëëëëëëëëëëëëf

OFF BIT TEXT: norm ON BIT TEXT: alarm

OFF BIT TEXT: norm ON BIT TEXT: alarm

OFF BIT TEXT: norm ON BIT TEXT: Alarm

OFF BIT TEXT: norm

ON BIT TEXT: alarm 


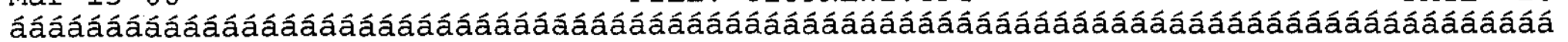

SCREEN \#046: DATA DISPLAY

SCREEN MEMORY USED: 182 BYTES

PREVIOUS MENU:

NEXT SCREEN:

PREVIOUS SCREEN :

DATA POSITION \#1:

REGISTER :

BIT NUMBER:

TEXT LENGTH:

DATA POSITION \#2:

REGISTER :

BIT NUMBER :

TEXT LENGTH:

DATA POSITION \#3:

REGISTER :

BIT NUMBER:

TEXT LENGTH:

DATA POSITION \#4:

REGISTER :

BIT NUMBER :

TEXT LENGTH:
040

048

UNLINKED

BIT DISPLAY

N20:25

5

5

BIT DISPLAY

$\mathrm{N} 20: 25$

6

5

OFF BIT TEXT:

ON BIT TEXT:

OFF BIT TEXT: ON BIT TEXT:

7

5

BIT DISPLAY

$\mathrm{N} 20: 25$

8

5 \{́丶龴ëëëëëëëëëëëëëëëëëë

- Recirc LoOp BD1ED 。

- FGM इD2DD。

- Leak ST\#4 SD3DD。

- U Shtdown DD4DP。

åëëëëëëëëëëëëëëëëëëë $f$

OFF BIT TEXT: norm

ON BIT TEXT: Alarm

OFF BIT TEXT: norm

ON BIT TEXT: Alarm

PAGE C 31 OF C 50 


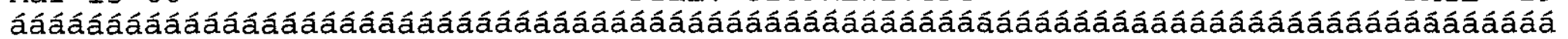

SCREEN \#048: DATA DISPLAY

SCREEN MEMORY USED: 178 BYTES

PREVIOUS MENU: $\quad 040$

NEXT SCREEN : $\quad 050$

PREVIOUS SCREEN : 046

DATA POSITION \#1: BIT DISPLAY

$\begin{array}{ll}\text { REGISTER: } & \text { N20:25 } \\ \text { BIT NUMBER: } & 9 \\ \text { TEXT LENGTH : } & 5\end{array}$

OFF BIT TEXT: ON BIT TEXT:

OFF BIT TEXT: ON BIT TEXT:

$\mathrm{N} 20: 25$

10

4

TEXT LENGTH:

DATA POSITION \#3:

REGISTER :

BIT NUMBER :

TEXT LENGTH:

DATA POSITION \#4:

REGISTER :

BIT NUMBER :

TEXT LENGTH :

N20:25

ON BIT TEXT:
BIT DISPLAY

$\mathrm{N} 20: 25$

11

5

OFF BIT TEXT: ON BIT TEXT:

norm

Alarm

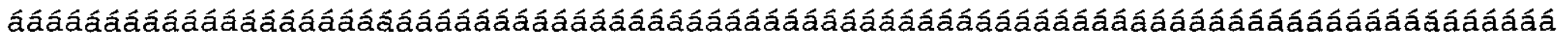

SCREEN \#050: DATA DISPLAY

乏̇ëëëëëëëëëëëëëëëëë;

SCREEN MEMORY USED: 139 BYTES

PREVIOUS MENU: UNLINKED

NEXT SCREEN : 052

OFF BIT TEXT: norm

Leak

- U-D Leak ck

- CGM FAULT

- Leak ST\#5

DEIDE。

DE2EE

$\circ$

048

åëëëëëëëëëëëëëëëëëëëf

DATA POSITION \#1: BIT DISPLAY

$\begin{array}{llll}\text { REGISTER : } & \text { N20:25 } & \text { OFF BIT TEXT: } & \text { norm } \\ \text { BIT NUMBER : } & 13 & \text { ON BIT TEXT: } & \text { Alarm } \\ \text { TEXT LENGTH : } & 5 & & \end{array}$

DATA POSITION \#2: BIT DISPLAY

$\begin{array}{llll}\text { REGISTER: } & \text { N20:25 } & \text { OFF BIT TEXT: } & \text { norm } \\ \text { BIT NUMBER : } & 14 & \text { ON BIT TEXT: } & \text { Alarm } \\ \text { TEXT LENGTH : } & 5 & & \end{array}$

DATA POSITION \#3: BIT DISPLAY

$\begin{array}{ll}\text { REGISTER : } & \text { N20:25 } \\ \text { BIT NUMBER: } & 15 \\ \text { TEXT LENGTH : } & 5\end{array}$

REGISTER :

TEXT LENGTH :
15
OFF BIT TEXT: ON BIT TEXT:

PAGE C 32 OF C 50 
ááááááááááááááâáááááááááăááááááááááááááááááááááááááááááááááááááááá

SCREEN \#052: DATA DISPLAY

SCREEN MEMORY USED: 182 BYTES

PREVIOUS MENU:

NEXT SCREEN : PREVIOUS SCREEN :

DATA POSITION \#1:

REGISTER :

BIT NUMBER:

TEXT LENGTH:

DATA POSITION \#2:

REGISTER :

BIT NUMBER:

TEXT LENGTH:

DATA POSITION \#3:

REGISTER :

BIT NUMBER:

TEXT LENGTH :

DATA POSITION \#4:

REGISTER :

BIT NUMBER :

TEXT LENGTH :
UNLINKED

053

UNLINKED

BIT DISPLAY

\{́ëëëëëëëëëëëëëëëëë ¡

- COB U33 Leak SD1DD

- Unused DD2DD 0

- Dilution tk DDЗDD。

- COB U33 ck DE4DD åëëëëëëëëëëëëëëëëëë $f$

OFF BIT TEXT: norm ON BIT TEXT: Alarm

OFF BIT TEXT: norm ON BIT TEXT: Alarm

OFF BIT TEXT: norm ON BIT TEXT: Alarm

OFF BIT TEXT: norm ON BIT TEXT: Alarm

PAGE C $\underline{33}$ OF C $\underline{50}$ 
SCREEN \#053: DATA DISPLAY

SCREEN MEMORY USED: 182 BYTES

$\begin{array}{ll}\text { PREVIOUS MENU : } & \text { UNLINKED } \\ \text { NEXT SCREEN : } & 054 \\ \text { PREVIOUS SCREEN : } & 052\end{array}$

DATA POSITION \#1: BIT DISPLAY

$\begin{array}{ll}\text { REGISTER : } & \mathrm{N} 20: 51 \\ \text { BIT NUMBER : } & 4 \\ \text { TEXT LENGTH : } & 5\end{array}$

DATA POSITION \#2: BIT DISPLAY

$\begin{array}{ll}\text { REGISTER : } & \mathrm{N} 20: 51 \\ \text { BIT NUMBER : } & 5 \\ \text { TEXT LENGTH : } & 5\end{array}$

DATA POSITION \#3: BIT DISPLAY

REGISTER :

BIT NUMBER:

TEXT LENGTH:

DATA POSITION \#4:

REGISTER :

BIT NUMBER:

TEXT LENGTH :

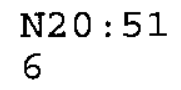

BIT DISPLAY

$\mathrm{N} 20: 51$

7

5

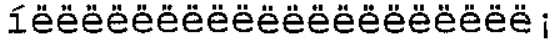

- U Flush Pres BDIDD 。

- U Recir Pres DD2DD。

- Recirc Press Б\$3DS。

- Pit U-A lk B\$45 ० åëëëëëëëëëëëëëëëëëëf

OFF BIT TEXT: norm ON BIT TEXT: Alarm

OFF BIT TEXT: norm ON BIT TEXT: Alarm

OFF BIT TEXT: norm ON BIT TEXT: Alarm

OFF BIT TEXT: norm ON BIT TEXT: Alarm 
SCREEN \#054: DATA DISPLAY

SCREEN MEMORY USED: 182 BYTES

\section{PREVIOUS MENU:}

NEXT SCREEN :

PREVIOUS SCREEN :

DATA POSITION \#1:

REGISTER :

BIT NUMBER:

TEXT LENGTH :

DATA POSITION \#2:

REGISTER :

BIT NUMBER :

TEXT LENGTH:

DATA POSITION \#3:

REGISTER :

BIT NUMBER:

TEXT LENGTH :

DATA POSITION \#4:

REGISTER :

BIT NUMBER :

TEXT LENGTH:

N20:51

$\mathrm{N} 20: 51$

10

5

BIT DISPLAY

N20:51

11

5
UNLINKED

055

053

BIT DISPLAY

8

5

BIT DISPLAY

N20:51

9

5

OFF BIT TEXT:

ON BIT TEXT:

OFF BIT TEXT:

ON BIT TEXT:

乏̇ëëëëëëëëëëëëëëëë i

- Pit U-A ck DDIDE

- Pit U-B lk DD2DD。

- Pit U-B ck БD3DE

- COB U-30 lk DD4DD 。

åëëëëëëëëëëëëëëëëëëë $f$

norm

Alarm

OFF BIT TEXT: norm

Alarm

ON BIT TEXT:

OFF BIT TEXT:

norm

ON BIT TEXT: Alarm 


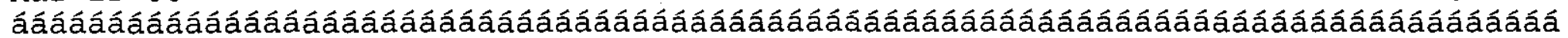

SCREEN \#055: DATA DISPLAY

SCREEN MEMORY USED: 182 BYTES

$\begin{array}{ll}\text { PREVIOUS MENU : } & \text { UNLINKED } \\ \text { NEXT SCREEN : } & 056 \\ \text { PREVIOUS SCREEN : } & 054\end{array}$

DATA POSITION \#1: BIT DISPLAY

$\begin{array}{ll}\text { REGISTER : } & \text { N20:51 } \\ \text { BIT NUMBER: } & 12 \\ \text { TEXT LENGTH : } & 5\end{array}$

DATA POSITION \#2: BIT DISPLAY

$\begin{array}{ll}\text { REGISTER : } & \text { N20:51 } \\ \text { BIT NUMBER : } & 13 \\ \text { TEXT LENGTH : } & 5\end{array}$

DATA POSITION \#3: BIT DISPLAY

REGISTER :

BIT NUMBER :

TEXT LENGTH :

DATA POSITION \#4:

REGISTER :

BIT NUMBER:

TEXT LENGTH:
N20:51

14

5

BIT DISPLAY

N20:51

15

5

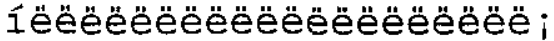

- COB U-30 ck DEIPB 。

- URecirc loss DE2DD。

- U Flush loss DD3DD。

- SY-B Leak al BP4DP 。

åëëëëëëëëëëëëëëëëëë $f$
OFF BIT TEXT: norm

ON BIT TEXT: Alarm
OFF BIT TEXT: norm ON BIT TEXT: Alarm
OFF BIT TEXT: norm ON BIT TEXT: Alarm
OFF BIT TEXT: norm

ON BIT TEXT: Alarm

PAGE C 36 OF C 50 


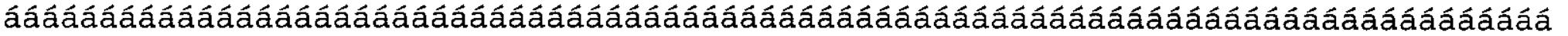

SCREEN \#056: DATA DISPLAY

SCREEN MEMORY USED: 178 BYTES

PREVIOUS MENU: $\quad$ UNLINKED

NEXT SCREEN:

PREVIOUS SCREEN : 055

DATA POSITION \#1: BIT DISPLAY

REGISTER :

BIT NUMBER:

TEXT LENGTH :

N20:52

0

4

DATA POSITION \#2: BIT DISPLAY

REGISTER :

BIT NUMBER:

TEXT LENGTH :

DATA POSITION \#3: BIT DISPLAY

REGISTER :

BIT NUMBER :

TEXT LENGTH:

$\mathrm{N} 20: 52$

2

5 \{́ëëëëëëëëëëëëëëëëë i

-WTR CAB TEMP DDID

'WFIE TEMP DE2D ०

- Unused DD3Б5

- Unused \$\$4Б口

åëëëëëëëëëëëëëëëëëëëf $\begin{array}{ll}\text { OFF BIT TEXT: } & \text { norm } \\ \text { ON BIT TEXT: } & \text { LOW }\end{array}$

OFF BIT TEXT:

ON BIT TEXT:

norm

LOW

DATA POSITION \#4: BIT DISPLAY
REGISTER :
$\mathrm{N} 20: 52$
BIT NUMBER:
TEXT LENGTH :
3
OFF BIT TEXT:
norm
ON BIT TEXT:
Alarm

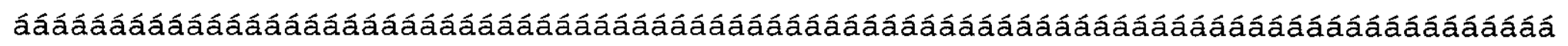

SCREEN \#057: DATA DISPLAY

SCREEN MEMORY USED: 110 BYTES

PREVIOUS MENU: UNLINKED

NEXT SCREEN : 058

PREVIOUS SCREEN :

DATA POSITION \#1: BIT DISPLAY

REGISTER :

BIT NUMBER:

TEXT LENGTH:

DATA POSITION \#2: BIT DISPLAY 乏̇ëëëëëëëëëëëëëëëëë i

- Exhst Online DD1DS。

- Unused

- Unused

- Unused

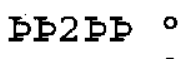

åëëe̋ëëëëëëëëëëëëëëëë $f$
OFF BIT TEXT: norm

ON BIT TEXT: Alarm
REGISTER :
$\mathrm{N} 20: 52$
BIT NUMBER:
6
OFF BIT TEXT:
norm
TEXT LENGTH :
5
ON BIT TEXT:
Alarm

PAGE C 37 OF C 50 


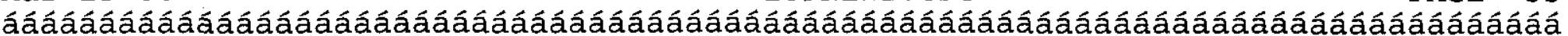

SCREEN \#058: DATA DISPLAY

İëëëëëëëëëëëëëëëëëë;

SCREEN MEMORY USED: 53 BYTES

PREVIOUS MENU: UNLINKED

NEXT SCREEN: UNLINKED

- Unused

$\mathrm{DP} \mathrm{DD} \circ$

PREVIOUS SCREEN :

057

DATA POSITION \#1: BIT DISPLAY
REGISTER :
$\mathrm{N} 20: 52$
BIT NUMBER:
8
OFF BIT TEXT:
norm
TEXT LENGTH :
5
ON BIT TEXT:
Alarm

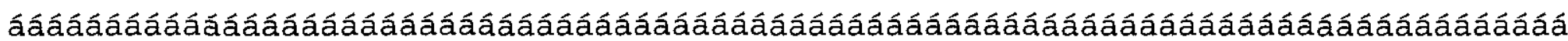

SCREEN \#100: ALARM 乏́ëëëëëëëëëëëëëëëëëë

SCREEN MEMORY USED: 68 BYTES

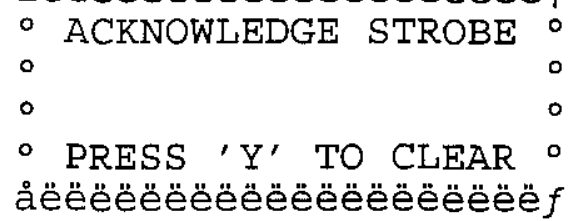

ACKNOWLEDGE ALARM TO REGISTER: N12:0

BIT NUMBER:

BIT WILL BE:

0

SET .

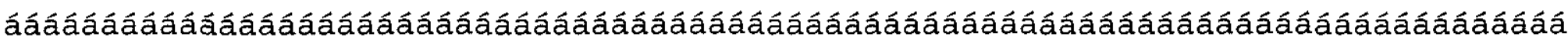

SCREEN \#101: ALARM

乏́ëëëëëëëëëëëëëëëëë ;

SCREEN MEMORY USED: 79 BYTES

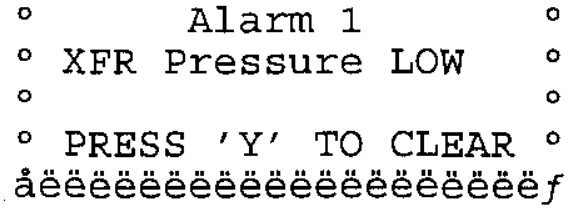

ACKNOWLEDGE ALARM TO REGISTER: N12:0

BIT NUMBER:

1

BIT WILL BE:

SET.

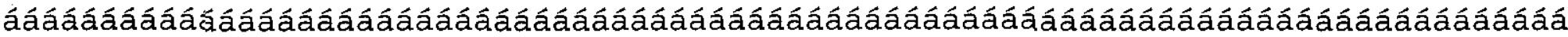

SCREEN \#102: ALARM

SCREEN MEMORY USED: 81 BYTES

\{́ëëëëëëëëëëëëëëëëëëë

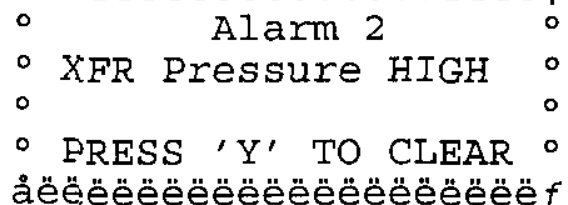

ACKNOWLEDGE ALARM TO REGISTER: N12:0

BIT NUMBER:

BIT WILL BE:

2

SET.

PAGE C 38 OF C 50 


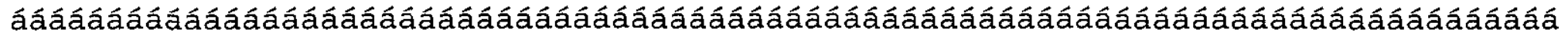

SCREEN \#103: ALARM

SCREEN MEMORY USED: 81 BYTES

ACKNOWLEDGE ALARM TO REGISTER: N12:0

BIT NUMBER:

BIT WILL BE: \{́ëëëëëëëëëëëëëëëëë;

- Alarm 3

- Flush Pressure HI

- Flush Pressure HI 0

- PRESS 'Y' TO CLEAR ०

åëëëëëëëëëëëëëëëëëëf

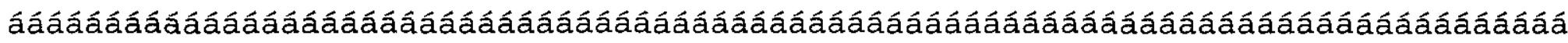

SCREEN \#104: ALARM

SCREEN MEMORY USED: 81 BYTES ட̇ëëëëëëëëëëëëëëëëë ;

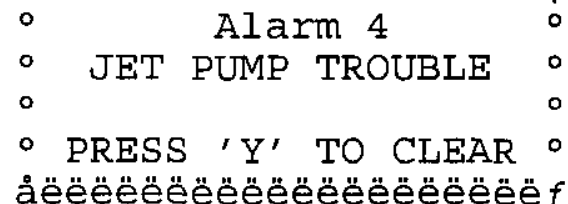

ACKNOWLEDGE ALARM TO REGISTER: N12:0

BIT NUMBER:

BIT WILL BE:

4

SET.

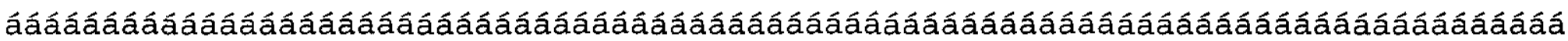

SCREEN \#105: ALARM

SCREEN MEMORY USED: 93 BYTES 1́ëëëëëëëëëëëëëëëëëë i

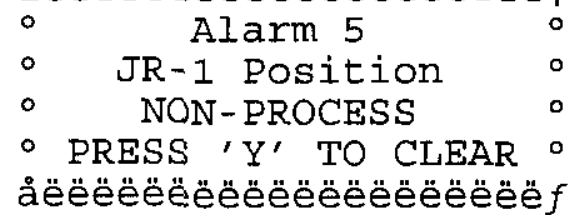

$\mathrm{N} 12: 0$

5

SET.

BIT WILL BE:

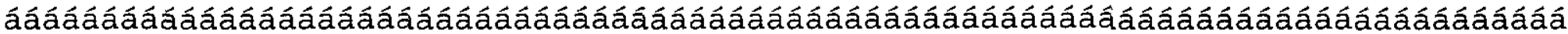

SCREEN \#106: ALARM

1́ëëëëëëëëëëëëëëëëëë ;

SCREEN MEMORY USED: 79 BYTES

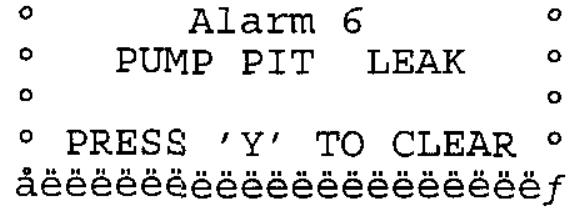

ACKNOWLEDGE ALARM TO REGISTER: N12:0

BIT NUMBER:

BIT WILL BE:

6

SET. 


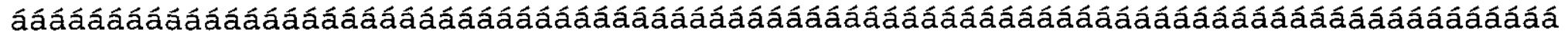

SCREEN \#107: ALARM

SCREEN MEMORY USED: 91 BYTES

ACKNOWLEDGE ALARM TO REGISTER: N12:0

BIT NUMBER:

BIT WILL BE : 乏́ëëëëëëëëëëëëëëëëëë

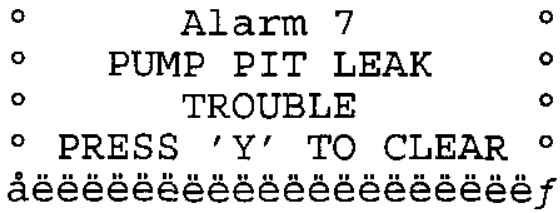

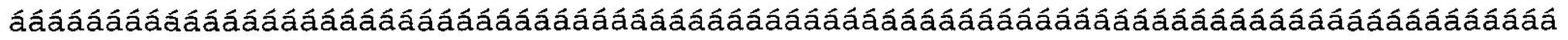

SCREEN \#108: ALARM

SCREEN MEMORY USED: 92 BYTES

乏́ëëëëëëëëëëëëëëëëë ;

$\circ$
$\circ$
$\circ$

ACKNOWLEDGE ALARM TO REGISTER: N12:0

BIT NUMBER:

BIT WILL BE:

8

SET.

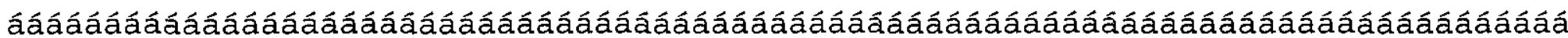

SCREEN \#109: ALARM

SCREEN MEMORY USED: 81 BYTES

\{́ëëëëëëëëëëëëëëëëëë i

- Alarm 9 o

-PIC Water Level LOW

- PRESS ' $Y$ ' TO CLEAR ○

åëëëëëëëëëëëëëëëëëëë $f$

ACKNOWLEDGE ALARM TO REGISTER: N12:0

BIT NUMBER: 9

BIT WILL BE:

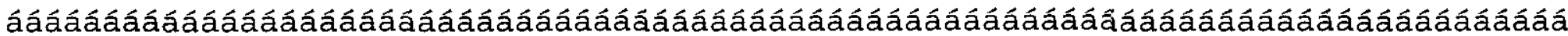

SCREEN \#110: ALARM

1̇ëëëëëëëëëëëëëëëëë i

SCREEN MEMORY USED: 81 BYTES

Alarm 10

- PLC Enclosure HI ०

- PRESS 'Y' TO CLEAR ○

åëëëëëëëëëëëëëëëëë $f$

ACKNOWLEDGE ALARM TO REGISTER: N12:0

BIT NUMBER :

BIT WILL BE:

10

SET. 


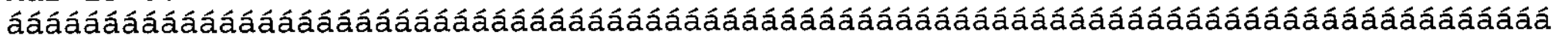

SCREEN \#111: ALARM

SCREEN MEMORY USED: 92 BYTES 1́ëëëëëëëëëëëëëëëëëë

-Air Compressor Temp 。

- HI

- PRESS 'Y' TO CLEAR ○

åëëëëëëëëëëëëëëëëëëf

ACKNOWLEDGE ALARM TO REGISTER: N12:0

BIT NUMBER :

11

BIT WILL BE:

SET.

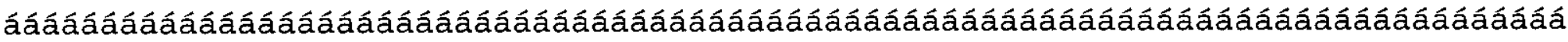

SCREEN \#112: ALARM

SCREEN MEMORY USED: 81 BYTES

\{́ëëëëëëëëëëëëëëëëëëëë

- JET PUMP SHUTDOWN

-

- PRESS 'Y' TO CLEAR ०

åëëëëëëëëëëëëëëëëëë $f$

ACKNOWLEDGE ALARM TO REGISTER: N12:0

BIT NUMBER :

12

BIT WILL BE:

SET.

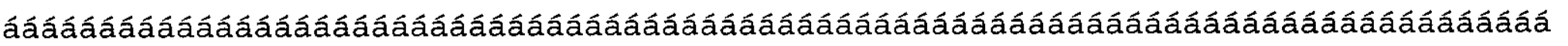

SCREEN \#113: ALARM

SCREEN MEMORY USED: 76 BYTES

1́ëëëëëëëëëëëëëëëëëë i

Alarm 13

- SGT LOW

- PRESS ' $Y$ ' TO CLEAR ०

åëëëëëëëëëëëëëëëëëëë $f$

ACKNOWLEDGE ALARM TO REGISTER: N12:0

BIT NUMBER:

13

BIT WILL BE:

SET.

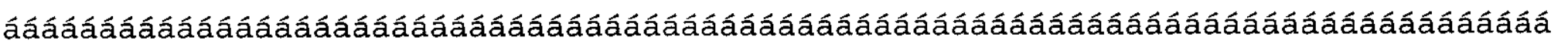

SCREEN \#114: ALARM

SCREEN MEMORY USED: 92 BYTES

\{

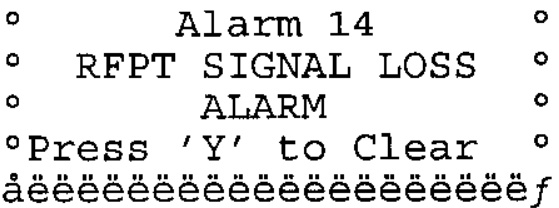

ACKNOWLEDGE ALARM TO REGISTER: N12:0

BIT NUMBER:

14

BIT WIIL BE:

SET.

PAGE C 41 OF C 50 


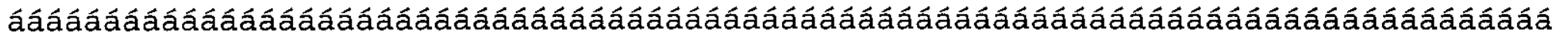

SCREEN \#115: ALARM

SCREEN MEMORY USED: 95 BYTES

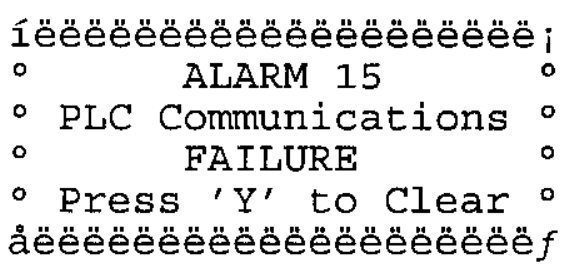

ACKNOWLEDGE ALARM TO REGISTER: N12:0

BIT NUMBER :

BIT WILL BE:
15

SET.

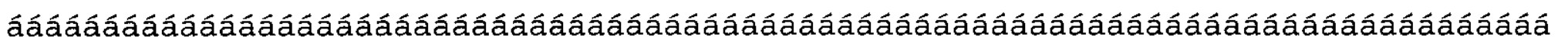

SCREEN \#116: ALARM

SCREEN MEMORY USED: 93 BYTES

ACKNOWLEDGE ALARM TO REGISTER: N12:1

BIT NUMBER:

BIT WILL BE:

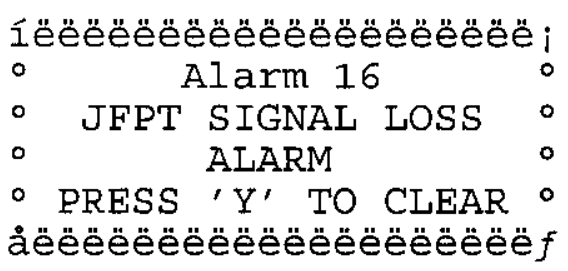

0

SET.

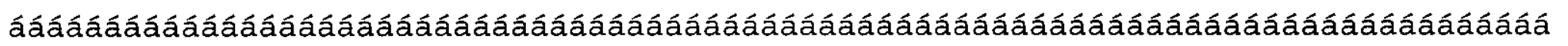

SCREEN \#117: ALARM

SCREEN MEMORY USED: 88 BYTES

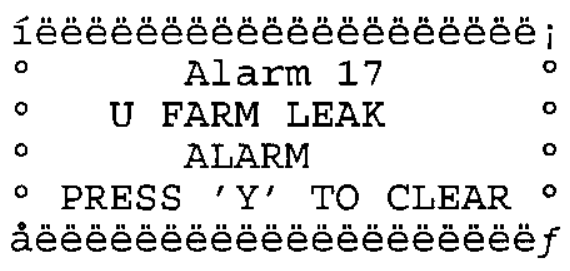

ACKNOWLEDGE ALARM TO REGISTER: N12:1

BIT NUMBER:

BIT WILL BE:

1

SET.

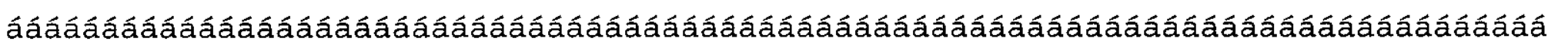

SCREEN \#118: ALARM

SCREEN MEMORY USED: 98 BYTES
BIT NUMBER:

BIT WILL BE:
ALARM TO REGISTER: N12:1
2
SET.

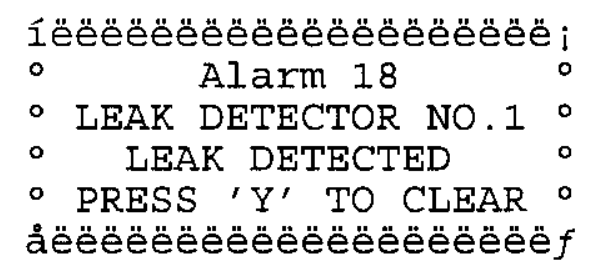

PAGEC 42 OFC 50 


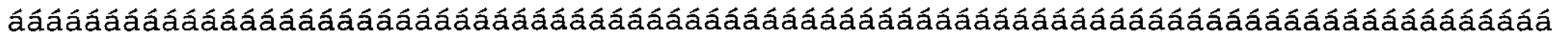

SCREEN \#119: ALARM

SCREEN MEMORY USED: 94 BYTES 1́ëëëëëëëëëëëëëëëëëëë

Alarm 19

LEAK DETECTOR NO.1

- TROUBLE

- PRESS Y TO CLEAR ०

åëëëëëëëëëëëëëëëëëëë $f$

ACKNOWLEDGE ALARM TO REGISTER: N12:1

BIT NUMBER:

BIT WILL BE:

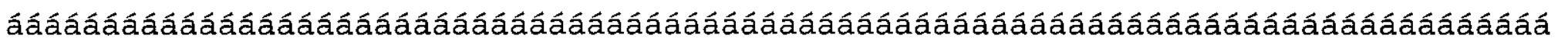

SCREEN \#120: ALARM

SCREEN MEMORY USED: 81 BYTES

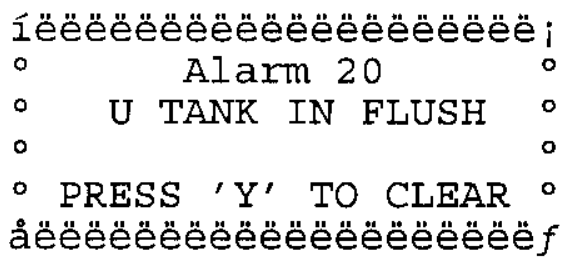

ACKNOWLEDGE ALARM TO REGISTER: N12:1

BIT NUMBER:

BIT WILL BE:

SET.

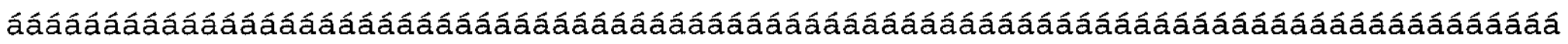

SCREEN \#121: ALARM

SCREEN MEMORY USED: 94 BYTES

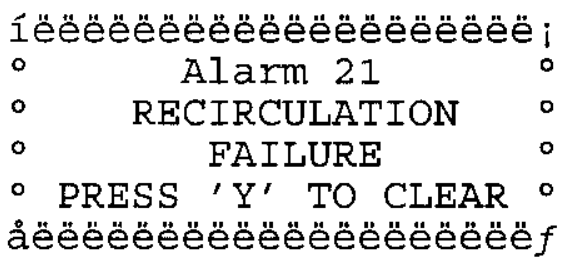

ACKNOWLEDGE ALARM TO REGISTER: N12:1

BIT NUMBER:

5

BIT WILL BE:

SET .

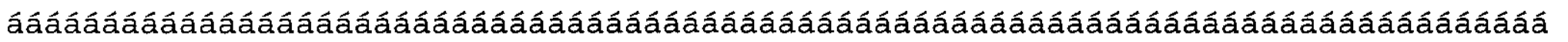

SCREEN \#122: ALARM

SCREEN MEMORY USED: 82 BYTES

1́ëëëëëëëëëëëëëëëëëë i

- Alarm 22 o

- FLAMMABLE GAS HIGH $\circ$

$\circ$

- PRESS 'Y' TO CLEAR ०

åëëëëëëëëëëëëëëëëëëf $f$

ACKNOWLEDGE ALARM TO REGISTER: N12:1

BIT NUMBER:

BIT WILL BE:

6

SET.

PAGE C 43 OF C 50 


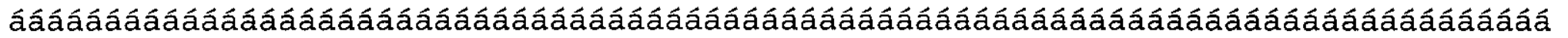

SCREEN \#123: ALARM

SCREEN MEMORY USED: 93 BYTES

ACKNOWLEDGE ALARM TO REGISTER: N12:1

BIT NUMBER:

BIT WILL BE :

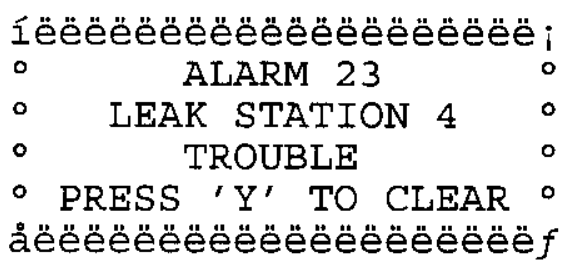

7

SET.

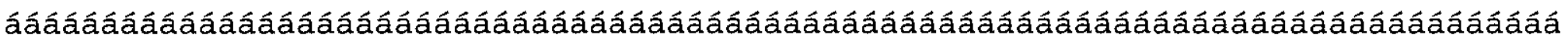

SCREEN \#124: ALARM

SCREEN MEMORY USED: 101 BYTES 1́ëëëëëëëëëëëëëëëëëë

- ALARM BIT 24 ०

- U TANK SHUTDOWN $\circ$

- timer ACTIVATED O

- PRESS 'Y' TO CLEAR ०

åëëëëëëëëëëëëëëëëëë $f$

ACKNOWLEDGE ALARM TO REGISTER: N12:1

BIT NUMBER:

BIT WILL BE: $\quad$ SET.

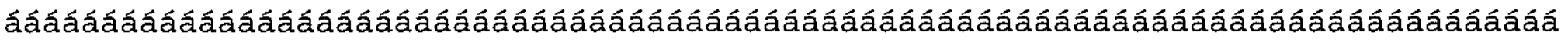

SCREEN \#125: ALARM

SCREEN MEMORY USED: 79 BYTES

\{́ëëëëëëëëëëëëëëëëë ;

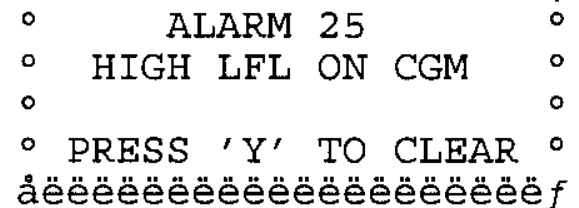

ACKNOWLEDGE ALARM TO REGISTER: N12:1

BIT NUMBER:

BIT WILL BE:

9

SET .

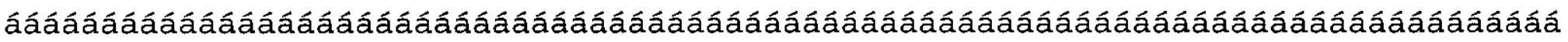

SCREEN \#126: ALARM

SCREEN MEMORY USED: 90 BYTES

ACKNOWLEDGE ALARM TO REGISTER: N12:1

BIT NUMBER:

BIT WILL BE:

10

SET. \{َëëëëëëëëëëëëëëëëëë;

$\begin{array}{lcl}\circ & \text { ALARM } 26 & \circ \\ \circ & \text { VALVE PIT U-C } & \circ \\ \circ & \text { LEAK } & \circ \\ \circ & \text { PRESS ' Y' TO CLEAR } \\ \text { åëëëëëëëëëëëëëëëëëëëf }\end{array}$




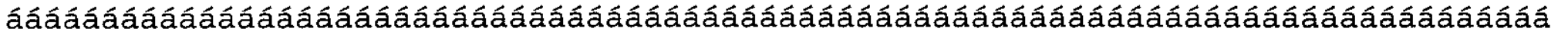

SCREEN \#127: ALARM

SCREEN MEMORY USED: 91 BYTES

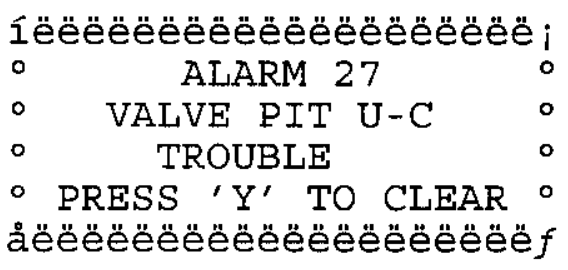

ACKNOWLEDGE ALARM TO REGISTER: N12:1

BIT NUMBER:

BIT WILL BE:
SET.

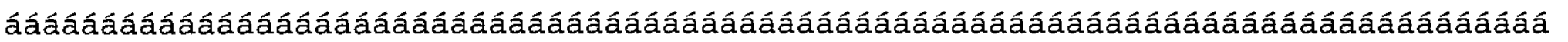

SCREEN \#128: ALARM

SCREEN MEMORY USED: 91 BYTES

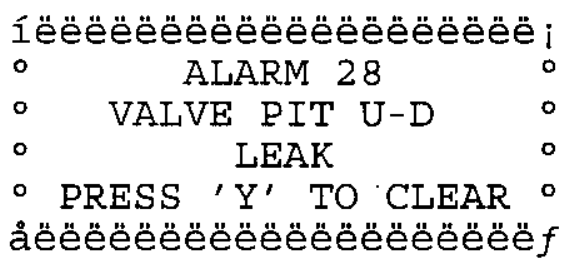

ACKNOWLEDGE ALARM TO REGISTER: N12:1

BIT NUMBER: 12

BIT WILL BE:
SET .

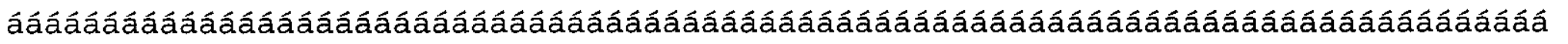

SCREEN \#129: ALARM

1ંëëëëëëëëëëëëëëëëëë i

SCREEN MEMORY USED: 91 BYTES

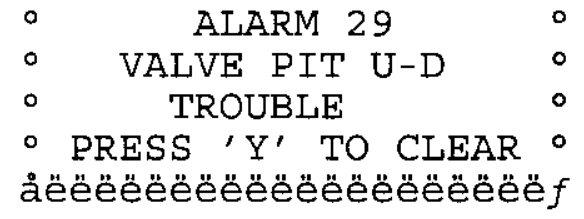

ACKNOWLEDGE ALARM TO REGISTER: N12:1

BIT NUMBER: 13

BIT WILL BE: SET.

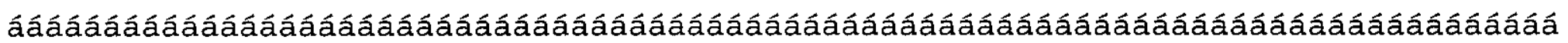

SCREEN \#130: ALARM

SCREEN MEMORY USED: 63 BYTES 1́ëëëëëëëëëëëëëëëëëëë

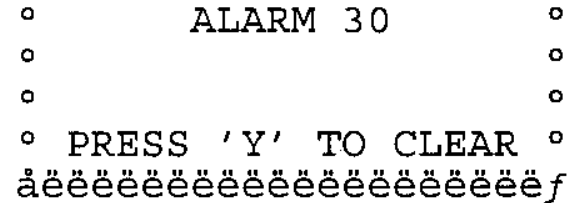

ACKNOWLEDGE ALARM TO REGISTER: N12:0

BIT NUMBER:

BIT WILL BE:

0

SET. 


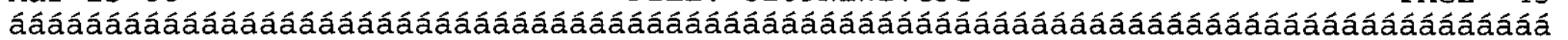

SCREEN \#131: ALARM

SCREEN MEMORY USED: 77 BYTES 1́ëëëëëëëëëëëëëëëëëëë ;

$\begin{array}{lcl}\circ & \text { ALARM } 31 & \circ \\ \circ & \text { CGM TROUBLE } & \circ \\ \circ & & \\ & & \end{array}$

åëëëëëëëëëëëëëëëëëëë $f$

ACKNOWLEDGE ALARM TO REGISTER: N12:1

BIT NUMBER: 14

BIT WILL BE:

SET.

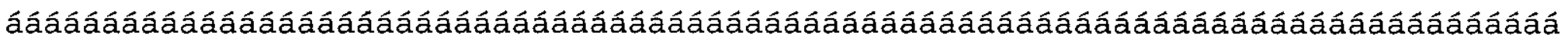

SCREEN \#132: ALARM

SCREEN MEMORY USED: 92 BYTES

1́ëëëëëëëëëëëëëëëëëëëe

$\circ$
$\circ$ AEAK STATION 5 ०

- TROUBLE O

- PRESS 'Y' TO CLEAR ०

åëëëëëëëëëëëëëëëëëë $f$

ACKNOWLEDGE ALARM TO REGISTER: N12:1

BIT NUMBER: 15

BIT WILL BE: SET.

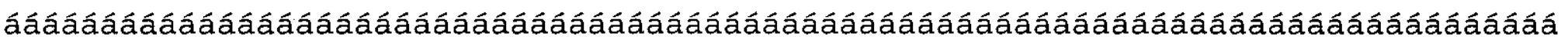

SCREEN \#133: ALARM

\{́ëëëëëëëëëëëëëëëëë ;

SCREEN MEMORY USED: 77 BYTES

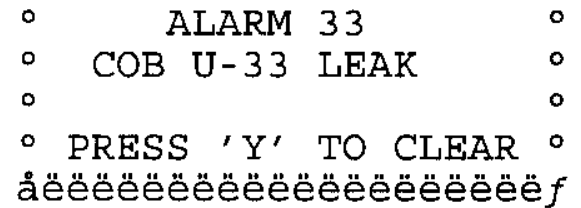

ACKNOWLEDGE ALARM TO REGISTER: N12:2

BIT NUMBER:

BIT WILL BE:

0

SET.

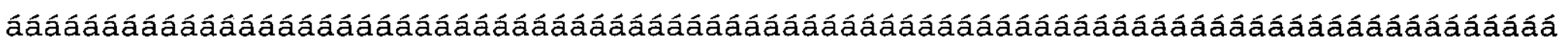

SCREEN \#134: ALARM

SCREEN MEMORY USED: 63 BYTES

ACKNOWLEDGE ALARM TO REGISTER: N12:2

BIT NUMBER:

BIT WILL BE:
1

SET .

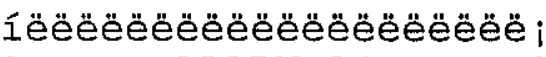

$\circ$

$\circ$

0

- PRESS 'Y' TO CLEAR ० åëëëëëëëëëëëëëëëëëëëf 


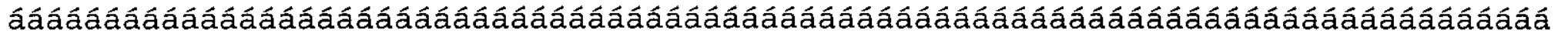

SCREEN \#135: ALARM

SCREEN MEMORY USED: 91 BYTES

ACKNOWLEDGE ALARM TO REGISTER: N12:2

BIT NUMBER:

BIT WILL BE:

2

SET.

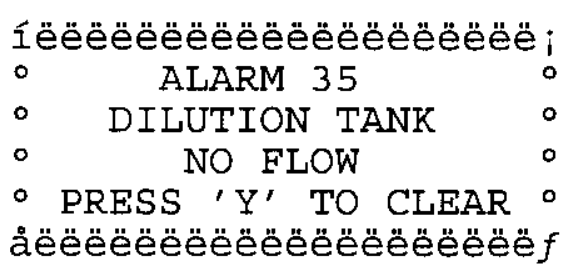

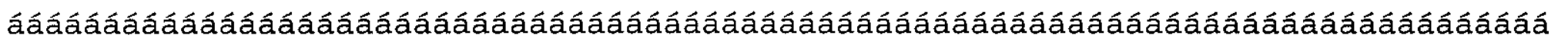

SCREEN \#136: ALARM

SCREEN MEMORY USED: 87 BYTES íëëëëëëëëëëëëëëëë i
$\circ \quad$ ALARM 36
$\circ$
COB U-33

N12: 2

3

SET.

BIT WILL BE:

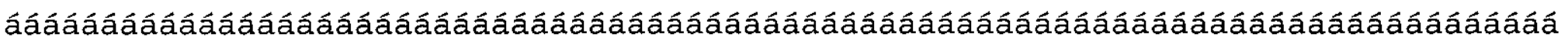

SCREEN \#137: ALARM

SCREEN MEMORY USED: 90 BYTES

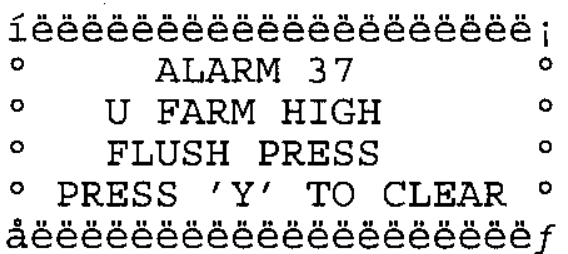

ACKNOWLEDGE ALARM TO REGISTER: N12:2

BIT NUMBER:

BIT WILL BE:

4

SET .

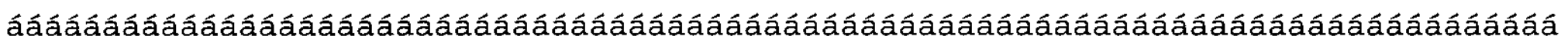

SCREEN \#138: ALARM

SCREEN MEMORY USED: 91 BYTES 1́ëëëëëëëëëëëëëëëëëëë

$\begin{array}{lll}\circ & \text { ALARM } 38 & \circ \\ \circ & \text { U FARM HIGH } & \circ \\ \circ & \text { RECIRC RRESS } & \circ \\ \circ & \text { PRESS 'Y' TO CLEAR } & \circ \\ \text { åëëëëëëëëëëëëëëëëëë } f\end{array}$

ACKNOWLEDGE ALARM TO REGISTER: N12:2

BIT NUMBER:

BIT WILL BE:

5

SET. 


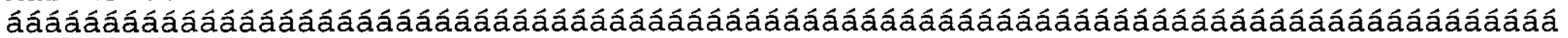

SCREEN \#139: ALARM

SCREEN MEMORY USED: 92 BYTES

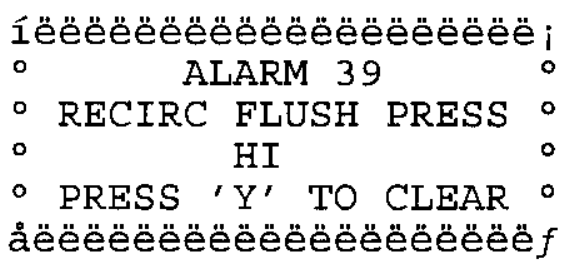

ACKNOWLEDGE ALARM TO REGISTER: N12:2

BIT NUMBER:

BIT WILL BE:
6

SET.

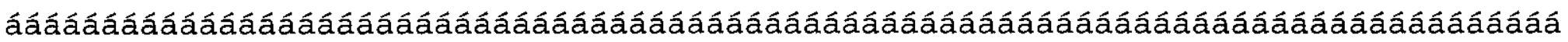

SCREEN \#140: ALARM
SCREEN MEMORY USED: 90 BYTES

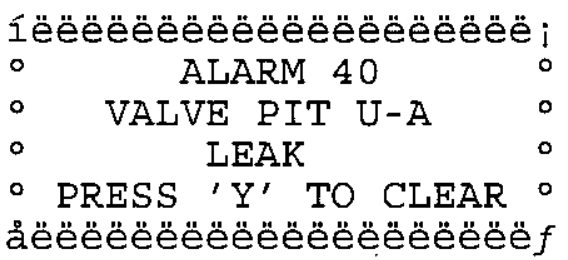

ACKNOWLEDGE ALARM TO REGISTER: N12:2

BIT NUMBER:

BIT WILL BE:
7

SET .

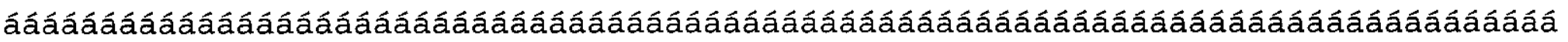

SCREEN \#141: ALARM

SCREEN MEMORY USED: 89 BYTES \{́ëëëëëëëëëëëëëëëëë ;

$\begin{array}{lcc}\circ & \text { ALARM } 41 & \circ \\ \circ & \text { VALVE PIT U-A } & \circ \\ \circ & \text { TROUBLE } & \circ \\ \circ & \text { PRESS 'Y' TO CLEAR } & \circ \\ \text { åëëëëëëëëëëëëëëëëëëëf }\end{array}$

ACKNOWLEDGE ALARM TO REGISTER: N12:2

BIT NUMBER:

BIT WILL BE:

8

SET .

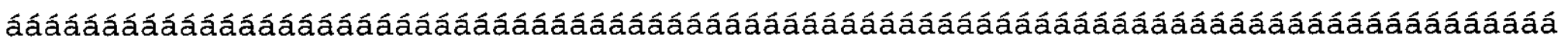

SCREEN \#142: ALARM

SCREEN MEMORY USED: 89 BYTES

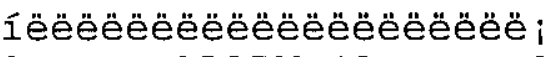

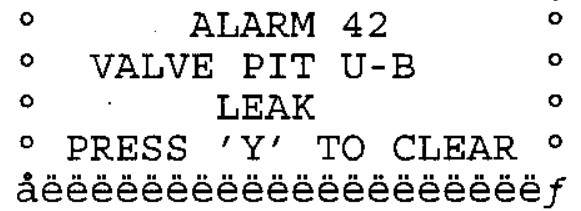

ACKNOWLEDGE ALARM TO REGISTER: N12:2

BIT NUMBER:

BIT WILL BE:

9

SET. 


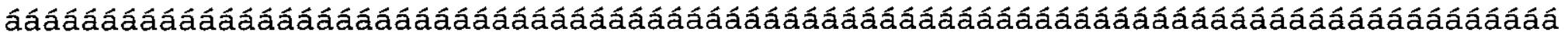

SCREEN \#143: ALARM

SCREEN MEMORY USED: 88 BYTES

ACKNOWLEDGE ALARM TO REGISTER: N12:2

BIT NUMBER :

BIT WILI BE:
10

SET. 1́ëëëëëëëëëëëëëëëëëëe ;

$\begin{array}{lcl}\circ & \text { ALARM } 43 & \circ \\ \circ & \text { VALVE PIT U-B } & \circ \\ \circ & \text { TROUBLE } & \circ \\ \circ & \text { PRESS 'Y' TO CLEAR } & \circ \\ \circ\end{array}$

åëëëëëëëëëëëëëëëëëë f

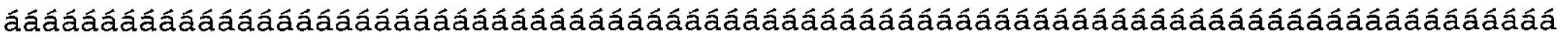

SCREEN \#144: ALARM

SCREEN MEMORY USED: 78 BYTES

ACKNOWLEDGE ALARM TO REGISTER: N12:2

BIT NUMBER:

BIT WILL BE:
11

SET.

(1)

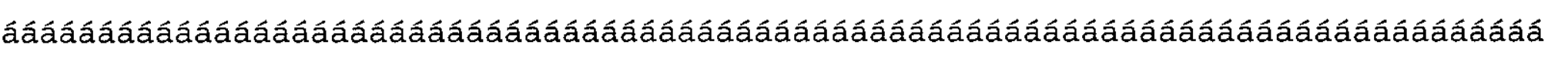

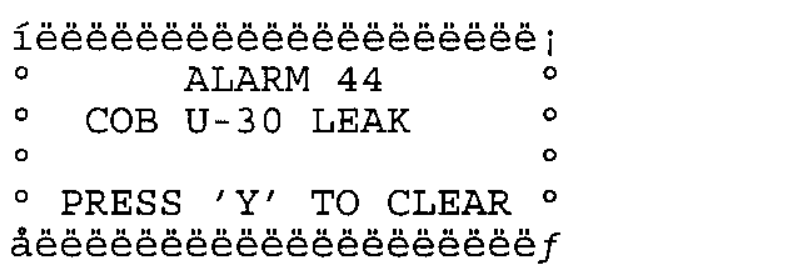

SCREEN \#145: ALARM

SCREEN MEMORY USED: 90 BYTES

íëëëëëëëëëëëëëëëë;
$\circ \quad$ ALARM 45
$\circ$
COB U-30

ACKNOWLEDGE ALARM TO REGISTER: N12:2

BIT NUMBER:

BIT WILL BE:

12

SET.

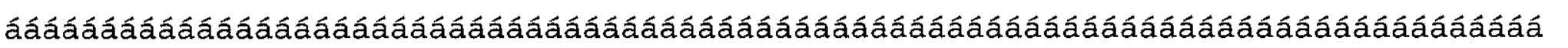

SCREEN \#146: ALARM

SCREEN MEMORY USED: 97 BYTES

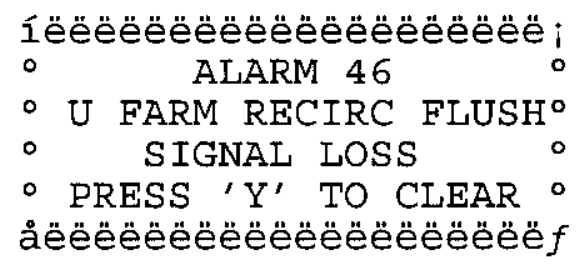

ACKNOWLEDGE ALARM TO REGISTER: N12:2

BIT NUMBER:

13

BIT WILL BE:

SET . 
SCREEN \#147: ALARM

SCREEN MEMORY USED: 97 BYTES

ACKNOWLEDGE ALARM TO REGISTER: N12:2

BIT NUMBER :

BIT WILL BE:

14

SET. 1́ëëëëëëëëëëëëëëëëëëë

- U FARM JUMPER FLUSH ${ }^{\circ}$

SIGNAL LOSS O

PRESS 'Y' TO CLEAR ०

åëëëëëëëëëëëëëëëëëë $f$

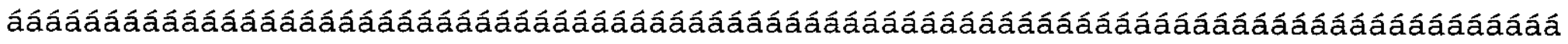

SCREEN \#148: ALARM

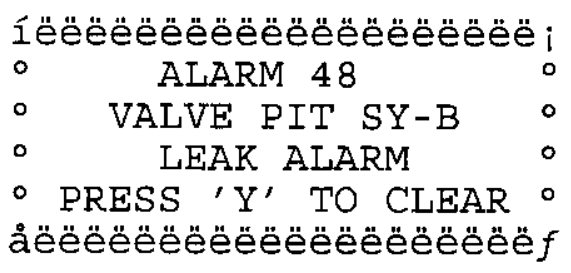

ACKNOWLEDGE ALARM TO REGISTER: N12:2

BIT NUMBER:

BIT WILL BE:
15

SET.

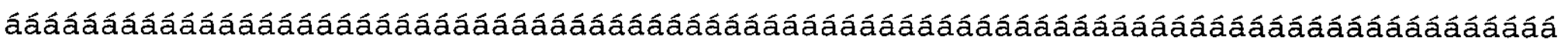

SCREEN \#149: ALARM

SCREEN MEMORY USED: 89 BYTES

ACKNOWLEDGE ALARM TO REGISTER: N12:3

BIT NUMBER:

BIT WILL BE:
0

SET . íëëëëëëëëëëëëëëëëëë;

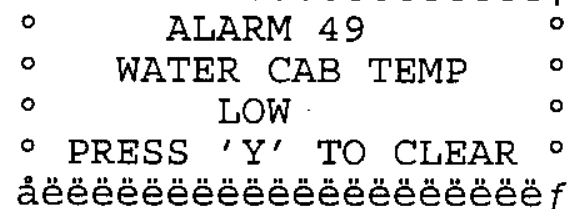

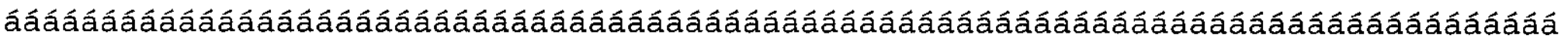

SCREEN \#150: ALARM

SCREEN MEMORY USED: 86 BYTES

ACKNOWLEDGE ALARM TO REGISTER: N12:3

BIT NUMBER:

BIT WILL BE: 